\title{
Experimentell-numerische Vorgehensweise zur Entwicklung von Probekörper-Setups für die Charakterisierung technischer Elastomere
}

\author{
Von der Fakultät für Maschinenbau der \\ Technischen Universität Chemnitz \\ genehmigte
}

\section{Dissertation}

zur Erlangung des akademischen Grades Doktoringenieur

(Dr.-Ing.)

vorgelegt

von Lars Kanzenbach M. Sc.

geboren am 30. Januar 1989 in Prenzlau

eingereicht am 21. August 2019

Gutachter:

Prof. Dr.-Ing. habil. Jörn Ihlemann

Prof. Dr.-Ing. Matthias Kröger

Tag der Verteidigung: 29. November 2019 
Persistente URN: http://nbn-resolving.de/urn:nbn:de:bsz:ch1-qucosa2-365142 


\section{Kurzfassung}

Für die Materialcharakterisierung und Parameteridentifikation von technischen Elastomeren werden homogene Probekörper benötigt. Eine besonders wichtige Beanspruchungsart ist dabei der einachsige Zug/Druck. Für Versuche dieser Art findet die Standard-Hantel Anwendung, die für kombinierte Zug-/Druckversuche geeignet ist. Allerdings lässt sich hier schon bei geringen Druckbelastungen ein inhomogener Messbereich detektieren. Ein Ziel dieser Arbeit besteht in der Entwicklung eines neuen und verbesserten Probekörpers, der für hochpräzise Zug-/Druckversuche geeignet ist. Im Gegensatz zur Standard-Hantel wird der für Messungen zugänglich gemachte homogene Messbereich deutlich verbessert. Darüber hinaus soll der Bereich der maximal erreichbaren Stauchung signifikant erhöht werden. Der Probekörper selbst weist dabei eine verhältnismäßig einfache Hantelgeometrie mit verlängertem Mittelteil auf. Durch ein spezielles Design der Halterungsgeometrie kann sowohl ein homogenes Verzerrungsfeld erreicht als auch eine hohe Knickstabilität gewährleistet werden. Die Grundidee besteht dabei darin, dass der Probekörper mit zunehmender Stauchung immer weiter mit der Halterungsgeometrie in Kontakt tritt und dadurch seine knickgefährdete Länge reduziert wird. Mit Hilfe eines speziellen Halterungsalgorithmus kann eine neue, verbesserte Halterungsgeometrie berechnet werden. Mit dem entwickelten Probekörper-Setup (bestehend aus Hantel- und Halterungsgeometrie) lassen sich dann eine Vielzahl phänomenologischer Eigenschaften von technischen Elastomeren wie PayneEffekt, Mullins-Effekt, Erholungs- und Relaxationsverhalten vorzugsweise bei extremen Stauchungen (bis zu $70 \%$ ) untersuchen.

Ein weiteres Ziel dieser Arbeit besteht in der Entwicklung eines Scherprobekörpers zur Realisierung präziser Schermessungen. Das Design soll dabei auf einem flächigen Probekörper (Elastomermatte) beruhen, um Alterungsuntersuchungen, Untersuchungen mit faserverstärkten Materialien und Versuche mit Vorreckungen realisieren zu können. Im Gegensatz zu herkömmlichen Scherprobekörpern soll dabei auf eine stoffschlüssige Verbindung mittels Kleben oder Anvulkanisieren aufgrund von Materialirritationen oder Schrumpf verzichtet werden. Im Rahmen dieser Arbeit wurde diesbezüglich ein spezielles Fixierdesign mit Stiften entwickelt, welches zur Ausbildung nahezu homogener Scherdeformationen führt. Damit lassen sich eine Vielzahl wichtiger Eigenschaften bei einer annähernd homogenen Scherdeformation untersuchen.

\section{Schlagworte}

Zug-/Druckmessungen, Probekörper-Setup, Probekörper Optimierung, Schermessungen, Schervorrichtung, homogener Messbereich, Gummiwerkstoffe, Gummi-Phänomenologie 


\section{Abstract}

Homogeneous test specimens are required for material characterization and model parameter identification. An important kind of loading is uniaxial tension/compression. For this, a standard dumbbell is available for combined tension-compression tests. But even for small compressive strains the standard dumbbell leads to an inhomogeneous stress state in the measuring zone. One aim of this work is the development of a new test specimen, which is suitable for high-precision tension/compression tests. In comparison to the standard dumbbell the homogeneity in the measuring zone is significantly improved. Furthermore, the range of maximal compression is increased substantially. The test specimen itself consists of a slender dumbbell structure. By a special design of the mounting geometry, homogeneous stress and strain fields as well as a high stability can be achieved. For an increasing compression, the test specimen comes into contact with the mounting geometry and the critical length is reduced. By means of dynamic analysis, the mounting geometry was calculated and optimized. This method is a powerful tool for developing new mounting geometries, by taking into account both the stability and the homogeneity characteristic. With the developed specimen-setup (consisting of dumbbell and mounting geometry), the phenomenological characteristics of rubber like Payne effect, Mullins effect, recovery and relaxation behavior can be investigated up to a compressive strain of $70 \%$.

Another aim of this work is the development of a shear specimen, which enables precision shear measurements for large shear values. The design is based on a planar test specimen (rubber mat) in order to enable ageing tests, tests with fibre-reinforced materials and tests with pre-stretching. In contrast to other shear specimens, a material-locking connection by gluing or vulcanizing sould be avoided in consequence of material irritation or shrinkage. For this, a special fixing design was developed, which enables a uniform initiation of shear deformation for different rubber thicknesses. Finally, the new shear specimen enables the investigation of typical rubber properties.

\section{Keywords}

Tension-/compression tests, specimen-setup, specimen optimization, shear tests, shear device, homogeneous measuring zone, rubber materials, rubber phenomenology 


\section{Vorwort}

Die vorliegende Arbeit entstand während meiner Tätigkeit als wissenschaftlicher Mitarbeiter an der Professur Festkörpermechanik der Technischen Universität Chemnitz.

Zuallererst möchte ich mich bei meinem Doktorvater Herrn Prof. Dr.-Ing. habil. Jörn Ihlemann für die Betreuung und Ermöglichung dieser Arbeit bedanken. Neben der Vielzahl an fachlichen Gesprächen hat seine individuelle Förderung und Unterstützung maßgeblich zum Erfolg dieser Arbeit beigetragen. Unsere Zusammenarbeit war auch gewinnbringend für meine persönliche Entwicklung in den vergangenen elf Jahren.

Weiterhin möchte ich mich bei Herrn Prof. Dr.-Ing. Matthias Kröger für das Interesse und die Übernahme des Zweitgutachtens dieser Arbeit bedanken.

Ein weiterer Dank gilt Herrn Dr.-Ing. habil. Martin Stockmann für die gute Zusammenarbeit und die fachlichen Diskussionen gerade im Bereich der experimentellen Mechanik.

Zudem möchte ich mich bei den Mitarbeitern der Professur Festkörpermechanik für die anregenden Diskussionen und die angenehme Arbeitsatmosphäre bedanken. In besonderer Weise gilt das für die Kollegen Sören Gelke, Clemens Schlomka, Dr.-Ing. Thomas Lehmann und Dr.-Ing. Ralf Landgraf.

Ein weiterer Dank richtet sich an die Projektpartner Vibracoustic GmbH \& Co. KG und FTI SE \& Co. KG. für die gemeinschaftliche Zusammenarbeit und die finanzielle Unterstützung dieses Projektes. Stellvertretend möchte ich mich bei Herrn Dr.-Ing. Rainer Klauke und Dr.-Ing. Christoph Naumann für die zahlreichen Projektbesprechungen bedanken, die zum Gelingen dieser Arbeit beigetragen haben.

Ein besonderer Dank richtet sich auch an Liliana Stoll für das Korrekturlesen dieser Arbeit.

Meine besondere Wertschätzung und mein Dank gilt meinen Eltern Gerno und Sonnlind Kanzenbach. Ich danke Euch, dass Ihr stets hinter mir steht, einen großen familiären Rückhalt bildet und für mich betet! Bedanken möchte ich mich auch bei meinen drei Geschwistern Anika, Lydia und Arne.

Mein größter Dank gilt allerdings meinem Herrn und Retter Jesus Christus. Ich habe ihm so vieles in meinem Leben zu verdanken. Besonders denke ich an seine Kraft, Zuversicht und Freude, die er mir immer wieder schenkt. Ich bin dankbar für seine Treue und die wunderbare Führung in den letzten Jahren.

\section{Lars Kanzenbach}





\section{Inhaltsverzeichnis}

Abkürzungs- und Symbolverzeichnis ix

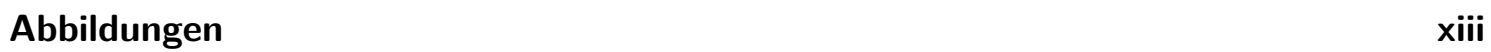

1 Einleitung 1

1.1 Motivation der Arbeit . . . . . . . . . . . . . . . . 1

1.2 Zielsetzung und Einordnung der eigenen Arbeit . . . . . . . . . . . 3

2 Grundlagen 5

2.1 Grundbegriffe der Kontinuumsmechanik . . . . . . . . . . . . 5

2.1 .1 Tensoralgebra ..................... 5

2.1.2 Verzerrungs- und Spannungstensoren . . . . . . . . . . . . 8

2.1.3 Trennung von Gestalt- und Volumenänderung . . . . . . . . . . . . 10

2.1.4 Hyperelastische Stoffgesetze mit quasi-inkompressibler Formulierung 11

2.2 Stand der Technik zu Elastomer-Probekörpern . . . . . . . . . . . . . . 13

2.2.1 Probekörper für einachsige Zug-/Druckversuche . . . . . . . . . . . 13

2.2.2 Probekörper für biaxiale Versuche . . . . . . . . . . . . . 14

2.2 .3 Scherprobekörper . . . . . . . . . . . . . . . 15

3 Probekörperdesign für hochpräzise Zug-/Druckmessungen 19

3.1 Motivation für die Entwicklung kombinierter Zug-/Druckprobekörper . . . . 19

3.2 Analytische Untersuchungen zur Knickstabilität von Stäben und Balken . . 20

3.2.1 Differentialgleichung der Balkenschwingung . . . . . . . . . . . . 20

3.2.2 Störkraftuntersuchungen am Euler-IV-Druckstab . . . . . . . . . . 22

3.2.3 Eigenfrequenzanalyse am Euler-Bernoulli-Balken . . . . . . . . . . . 27

3.2.4 Erweiterung der Eigenfrequenzanalyse für viskoelastische Strukturen 31

3.3 FE gestützte Entwicklung eines Zug-/Druckprobekörpers . . . . . . . . . . 355

3.3.1 Grundidee des Halterungsdesigns . . . . . . . . . . . . . 355

3.3.2 Algorithmus zur Berechnung von Halterungsgeometrien . . . . . . 38 
3.3.3 Parameterstudie zur Halterungsberechnung . . . . . . . . . . . 39

3.3.4 Fehler- und Instabilitätsmaße . . . . . . . . . . . . . . . . . 41

3.3.5 Optimierung der Probekörperlänge . . . . . . . . . . . . . 4 46

3.3.6 Formoptimierung der Halterungskontur . . . . . . . . . . . . 555

3.3.7 Erweiterung des Probekörperdesigns für extreme Stauchungen . . . . 61

3.4 Experimentelle Validierung des neuen Probekörper-Setups . . . . . . . . . 70

3.4.1 Versuchsaufbau des Probekörper-Setups in der Prüfmaschine . . . . 70

3.4.2 Erweiterte Prüfmaschinensteuerung . . . . . . . . . . 72

3.4.3 Voruntersuchungen und Qualitätsprüfungen . . . . . . . . . 73

3.4.4 Phänomenologische Untersuchung technischer Elastomere . . . . . . 81

4 Entwicklung eines Scherprobekörpers für präzise Schermessungen 89

4.1 Grundlegendes zu einfachen Scherversuchen . . . . . . . . . . . . . . . 899

4.2 Motivation für die Verwendung flächiger Scherprobekörper zur Realisierung präziser Schermessungen . . . . . . . . . . . . . . . . . . 91

4.3 Grundproblem Lasteinleitung . . . . . . . . . . . . . . . . . . . . 91

4.4 Numerische Entwicklung einer Schervorrichtung zur Realisierung präziser Schermessungen . . . . . . . . . . . . . . . . 9 93

4.4.1 Fehler- und Inhomogenitätsmaße . . . . . . . . . . . . . 93

4.4 .2 Untersuchung von Klemmdesigns . . . . . . . . . . . . . . . 999

4.4.3 Numerische Entwicklung eines Scherprobekörpers mit formschlüssiger Lasteinleitung . . . . . . . . . . . . . . . 103

4.5 Experimentelle Realisierung einer Schervorrichtung mit formschlüssiger Lasteinleitung . . . . . . . . . . . . . . . . . . 107

4.5.1 Versuchsaufbau der Schervorrichtung . . . . . . . . . . . . 107

4.5.2 Technologische Überlegungen zur Locherzeugung in Elastomermatten 108

4.5.3 Schermessungen mit Elastomermatten . . . . . . . . . . . . . 109

5 Anwendungsbeispiele für die entwickelten homogenen Probekörper 115

5.1 Homogene Standardversuche zur Identifikation von Materialparametern . . 115

5.2 Untersuchung des ratenabhängigen Materialverhaltens von Elastomeren . . 120

5.3 Implementierung des Probekörper-Setups in den Industriealltag . . . . . . . 126

6 Zusammenfassung und Ausblick 131

A Anhang

Literatur $\quad[139$ 


\title{
Abkürzungs- und Symbolverzeichnis
}

\author{
Abkürzungen \\ DIC Englisch für Digital Image Correlation \\ EPDM Englisch für Ethylene Propylene Diene Monomer \\ FEM Finite-Elemente-Methode \\ FKM $\quad$ Fluorkautschuk \\ GOM Gesellschaft für optische Messtechnik \\ Morph Englisch für Model of Rubber Phenomenology \\ NR Englisch für Natural Rubber \\ PBC Englisch für Periodic Boundary Condition \\ RVE Repräsentatives-Volumen-Element \\ TARRC Englisch für Tun Abdul Razak Research Centre
}

\section{Allgemeine Kennzeichnung}

Symbol Bedeutung

\begin{tabular}{ll}
\hline$X_{a b}$ & Tensorkoeffizienten \\
{$\left[X_{a b}\right]$} & Koeffizientenmatrix \\
$X$ & Tensor nullter Stufe (Skalar) \\
$X_{A}$ & Eigenwerte eines Tensors \\
$\widetilde{X}$ & Größe in der Referenzkonfiguration \\
$\mathrm{d} X$ & Differential (infinitesimal) \\
$\dot{X}$ & Substantielle Zeitableitung von skalaren Größen \\
$\underline{X}, \underline{\underline{X}}$ & Tensor erster und zweiter Stufe \\
$\underline{\underline{\underline{X}}}, \underline{\underline{A}}$ & Transponierter und inverser Tensor \\
$\underline{\underline{\underline{X}}}, \underline{\underline{\underline{X}}}$ & Symmetrischer und antisymmetrischer Anteil eines Tensors \\
$\underline{\underline{\underline{X}}}, \underline{\underline{\underline{X}}}$ & Hydrostatischer und deviatorischer Anteil eines Tensors \\
$\underline{\underline{\underline{X}}}, \underline{\underline{X}}$ & Volumen- und Gestaltänderungsanteil eines Tensors \\
$\underline{\underline{S}}((\underline{\underline{X}})), S^{-1}((\underline{\underline{X}}))$ & Übertragungsoperator und inverser Übertragungsoperator
\end{tabular}




\section{Produkte}

Symbol

Bedeutung

$\underline{\underline{X}} \cdot \underline{\underline{Y}}$

Einfaches Punktprodukt

$\underline{\underline{X}} \cdot \underline{\underline{Y}}$

Doppeltes Punktprodukt

$\underline{\underline{X}} \circ \underline{\underline{Y}}$

Dyadisches oder tensorielles Produkt

\section{Skalare}

Symbol

Bedeutung

\begin{tabular}{|c|c|}
\hline$A$ & Fläche \\
\hline$\alpha$ & Winkel, Gewichtung \\
\hline$\ddot{\alpha}$ & Winkelbeschleunigung \\
\hline$c_{i j}$ & Materialparameter im hyperelastischen Stoffgesetz \\
\hline$\gamma$ & Innere Dehnung \\
\hline$d$ & Durchmesser \\
\hline$\delta_{a b}$ & Kronecker-Symbol \\
\hline$\delta W^{\mathrm{nc}}$ & Virtuelle Arbeit \\
\hline$\Delta l$ & Längenänderung \\
\hline$E$ & Elastizitätsmodul \\
\hline$\varepsilon$ & Dehnung \\
\hline$\zeta$ & Dimensionslose Koordinate \\
\hline$\eta$ & Spezifische Entropie, Viskosität \\
\hline$\eta_{\mathrm{I}}, \eta_{\mathrm{s}}, \eta_{\sigma}$ & Lokales Fehlermaß \\
\hline$\eta_{\mathrm{T}}$ & Globales Fehlermaß \\
\hline$f$ & Frequenz, Zielfunktion, Fehlergröße \\
\hline$F$ & Kraft \\
\hline$G$ & Schubmodul \\
\hline$h$ & Höhe \\
\hline$I$ & Flächenträgheitsmoment \\
\hline$I_{1}, I_{2}, I_{3}$ & Hauptinvarianten \\
\hline$\theta$ & Absolute Temperatur \\
\hline$J$ & Massenträgheitsmoment \\
\hline$J_{1}, J_{2}, J_{3}$ & $J$-Invarianten \\
\hline$\iota$ & Größe der Imperfektion \\
\hline$K$ & Kompressionsmodul \\
\hline$\kappa$ & Instabilitätsmaß \\
\hline$l$ & Länge \\
\hline$L$ & Lagrangefunktion \\
\hline
\end{tabular}


$\lambda$

$m$

M

$\mu$

$n$

$N$

$\nu$

$\xi_{\mathrm{s}}$

$p$

$\varphi$

$\psi$

$q$

$Q$

$r$

$\rho$

$s$

$S$

$\bar{\sigma}_{\text {eqv }}$

$t$

T

$\tau$

$u$

$v$

V

$w$

$\dot{w}$

$\ddot{w}$

$\omega$

$x, y, z$

Eigenwert, Streckung

Masse

Moment

Streckungsfaktor, Reibung

Anzahl der Iterationsschritte

Normalkraft

Querkontraktionszahl

Inhomogenitätsmaß

Hydrostatischer Druck

Eigenform, Winkel

Freie Helmholtz-Energie

Belastungsintensität, generalisierte Koordinate

Querkraft

Radius

Dichte

Schermaß

Störkraft

Mittelwert

Zeit, Dicke

Kinetische Energie

Relaxationszeit

Verschiebung

Verschiebung, Grad der Verzerrung

Potentielle Energie

Verschiebung

Geschwindigkeit

Beschleunigung

Eigenfrequenz

Kartesische Koordinaten

\section{Vektoren}

Symbol

Bedeutung

$\begin{array}{ll}\underline{e}_{a} & \text { Basisvektor } \\ \underline{F} & \text { Kraftvektor } \\ \underline{n} & \text { Normaleneinheitsvektor } \\ \underline{\nabla} & \text { Nabla-Operator } \\ \underline{q} & \text { Wärmestromdichte } \\ \underline{r} & \text { Ortsvektor }\end{array}$


$\underline{s}$

$\underline{u} \quad$ Verschiebungsvektor

v Geschwindigkeitsvektor

\section{Tensoren zweiter Stufe}

Symbol Bedeutung

$\begin{array}{ll}\underline{\underline{b}} & \text { Linker Cauchy-Green-Tensor } \\ \underline{\underline{C}} & \text { Rechter Cauchy-Green-Tensor } \\ \underline{\underline{D}} & \text { Tensor der Formänderungsgeschwindigkeit } \\ \underline{\underline{\gamma}} & \text { Greenscher Verzerrungstensor } \\ \underline{\underline{\varepsilon}} & \text { Almansischer Verzerrungstensor } \\ \underline{\underline{F}} & \text { Deformationsgradient } \\ \underline{\underline{h}} & \text { Eulerscher Hencky-Tensor } \\ \underline{\underline{H}} & \text { Lagrangescher Hencky-Tensor } \\ \underline{\underline{I}} & \text { Einheits- oder Metriktensor } \\ \underline{\underline{L}} & \text { Geschwindigkeitsgradient } \\ \underline{\underline{\sigma}} & \text { Cauchy-Spannungstensor } \\ \underline{\underline{S}} & \text { Differenz zweier Spannungstensoren } \\ \underline{\underline{\widetilde{T}}} & \text { 1. Piola-Kirchhoff-Spannungstensor } \\ \underline{\underline{\underline{U}}} & 2 . \text { Piola-Kirchhoff-Spannungstensor } \\ \underline{\underline{V}} & \text { Rechter Strecktensor } \\ \underline{\underline{W}} & \text { Linker Strecktensor } \\ \underline{\underline{W}} & \text { Wirbeltensor }\end{array}$




\section{Abbildungen}

1.1 Zug-/Druckprobekörper (links) Probengeometrie (Abbildung aus der Arbeit von Lion 2000, S. 73) und (rechts) FE-Simulation mit Auswertung (Abbildungen aus der Veröffentlichung von Alshuth u. a. 2007)

2.1 Gegenüberstellung verschiedener Probekörper für einachsige Zug-/Druckversuche. Die Abbildung ist in leicht modifizierter Form Kanzenbach u.a. (2018) entnommen. Wiederverwendung mit Genehmigung. ㅇ 2018 Elsevier

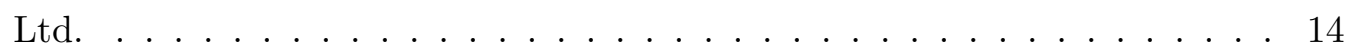

2.2 Biaxialer Zugprobekörper (Abbildung aus der Veröffentlichung von Seibert u. a. 2014) . . . . . . . . . . . . . . . . . 15

2.3 Gegenüberstellung zweier Scherprobekörper (links) zylindrischer DoubleShear-Probekörper (Abbildung aus der Veröffentlichung von Besdo u.a. 2003) und (rechts) flächiger Scherprobekörper von der Firma Akzo Nobel (Abbildung aus der Arbeit von Ihlemann 2003) . . . . . . . . . . . . 16

3.1 Freikörperbild eines infinitesimalen Balkenelements . . . . . . . . . . . . 21

3.2 Störgröße $S$ als Maß zum Erreichen der kritischen Kraft (Simulationsergeb-

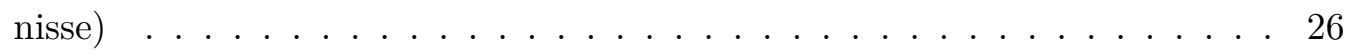

3.3 Darstellung der normierten Verschiebungsverläufe unter Ausnutzung der Symmetrie (Simulationsergebnisse, die Kurven schwarz und grün fallen zusammen) . . . . . . . . . . . . . . . . . . . 26

3.4 Darstellung der absoluten Eigenfrequenzverläufe eines Euler-Bernoulli-Balkens über der Dehnung $\varepsilon$ aufgrund der Normalkräfte (Simulationsergebnisse) 30

3.5 Darstellung der normierten Eigenfrequenzverläufe eines Euler-Bernoulli-Balkens über der Dehnung $\varepsilon$ aufgrund der Normalkräfte (Simulationsergebnis-

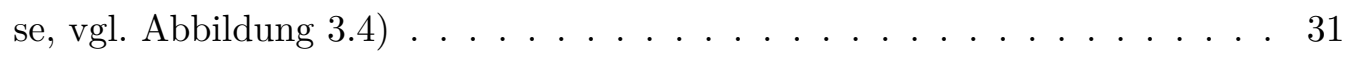

3.6 Darstellung der absoluten Eigenfrequenzverläufe eines viskoelastischen EulerBernoulli-Balkens zur Überprüfung der zwei Grenzfälle (Simulationsergebnisse, die ersten beiden Kurven und die letzten beiden Kurven fallen zu-

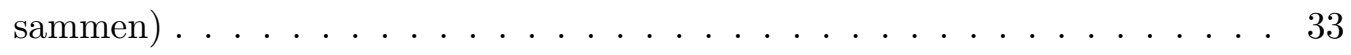

3.7 Darstellung der absoluten Eigenfrequenzverläufe eines viskoelastischen EulerBernoulli-Balkens unter Variation der Viskosität (Simulationsergebnisse, vgl. Abbildung 3.6, die letzten beiden Kurven fallen zusammen) . . . . . . . 34 
3.8 Modalanalyse zur Untersuchung der Eigenfrequenzverläufe der StandardHantel für verschiedene Ausgangslängen (Simulationsergebnisse vgl. Tabelle 3.1. Abbildung in Anlehnung an die Arbeit von Naumann 2010) . . . . . . . 36

3.9 Grundidee des Halterungsalgorithmus (Simulationsergebnisse vgl. Tabelle 3.1, Abbildung in Anlehnung an die Arbeit von Naumann 2010) . . . . . 37

3.10 Algorithmus zur Berechnung von Halterungskonturen (Abbildung aus der Arbeit von Naumann 2010) . . . . . . . . . . . . . . . 38

3.11 Parameterstudie zur Eigenfrequenzvorgabe für die Halterungsberechnung (Simulationsergebnisse vgl. Tabelle 3.1) . . . . . . . . . . . 39

3.12 Parameterstudie für die Halterungskonturen (Simulationsergebnisse) . . . . 40

3.13 Spannungs-Dehnungsdiagramm für die verschiedenen Halterungskonturen (Simulationsergebnisse vgl. Tabelle 3.1 . . . . . . . . . . . . . 41

3.14 Darstellung des globalen Fehlermaßes $\eta_{\mathrm{T}}$ für die verschiedenen Halterungskonturen (Simulationsergebnisse vgl. Tabelle 3.1 . . . . . . . . . 42

3.15 Darstellung des lokalen Fehlermaßes $\eta_{\sigma}$ für die verschiedenen Halterungskonturen bei einer Stauchung von $40 \%$ (Abbildung in Anlehnung an die Arbeit von Naumann 2010) . . . . . . . . . . . . . . . 44

3.16 Darstellung der Verschiebung $u_{\mathrm{L}}$ und $u_{\mathrm{R}}$ für verschiedene Sonderfälle . . . 45

3.17 Darstellung des Instabilitätsmaßes $\kappa$ für die verschiedenen Halterungskonturen (Simulationsergebnisse vgl. Tabelle $3.1 \ldots \ldots$

3.18 Ablaufplan für die Optimierung der Probekörperlänge . . . . . . . . . . . 47

3.19 Darstellung der konstanten Eigenfrequenzvorgaben für unterschiedliche Probekörperlängen $l_{0} \in[40,100] \mathrm{mm}$ sowie der aus dem Halterungsalgorithmus berechneten Eigenfrequenzverläufe (Simulationsergebnisse vgl. Tabelle 3.1) . 48

3.20 Darstellung der identischen asymptotischen Eigenfrequenzvorgaben für unterschiedliche Probekörperlängen $l_{0} \in[40,100] \mathrm{mm}$ sowie der aus dem Halterungsalgorithmus berechneten Eigenfrequenzverläufe (Simulationsergebnisse vgl. Tabelle $3.1 \quad \ldots \ldots \ldots$

3.21 Gegenüberstellung der berechneten Halterungskonturen die aus den vorgegebenen Eigenfrequenzverläufen ermittelt worden sind (Simulationsergeb-

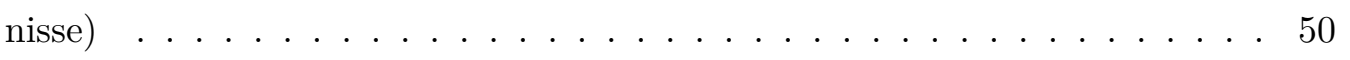

3.22 Darstellung der Zielfunktionsanteile $\eta_{\mathrm{T}}$ und $\kappa$ für konstante Eigenfrequenzvorgaben (Simulationsergebnisse vgl. Tabelle 3.1 Gewichtung $\alpha=0.6$ ) . . 51

3.23 Darstellung der Zielfunktion $f$ für konstante Eigenfrequenzvorgaben (Simulationsergebnis vgl. Tabelle $3.1 \ldots \ldots \ldots 5$

3.24 Darstellung der Zielfunktionsanteile $\eta_{\mathrm{T}}$ und $\kappa$ für identische asymptotische Eigenfrequenzvorgaben (Simulationsergebnisse vgl. Tabelle 3.1. Gewichtung

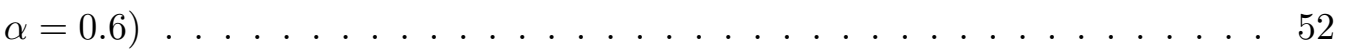


3.25 Darstellung der Zielfunktion $f$ für identische asymptotische Eigenfrequenzvorgaben (Simulationsergebnis vgl. Tabelle $3.1 \ldots \ldots$. . . . . . . 5 53

3.26 Darstellung des lokalen Fehlermaßes $\eta_{\sigma}$ für verschiedene Probekörperlängen $l_{0} \in[100,30] \mathrm{mm}$ bei einer Stauchung von $40 \% \ldots \ldots$. . . . . . 54

3.27 Ablaufplan für die Formoptimierung der Halterungskontur . . . . . . . . . . 56

3.28 Iterationsschritte des Simplex-Verfahrens . . . . . . . . . . . . . . 56

3.29 Halterungen aus der Parameterstudie (Simulationsergebnisse) . . . . . . . . 57

3.30 Lösungen der Optimierung 1 und 2 (Simulationsergebnisse) . . . . . . . . . 57

3.31 Normierte Eigenfrequenzverläufe für die Halterungsberechnung (Simulationsergebnisse vgl. Tabelle 3.1, vgl. Abbildung 3.11 . . . . . . . . . . . 57

3.32 Lösungen der Optimierung 3 bis 5 (Simulationsergebnisse) . . . . . . . . 58

3.33 Gegenüberstellung aller Konturen (Simulationsergebnisse) . . . . . . . . . 58

3.34 Darstellung des globalen Fehlermaßes $\eta_{\mathrm{T}}$ für die verschiedenen Halterungskonturen (Simulationsergebnisse vgl. Tabelle 3.1, vgl. Abbildung 3.14 . . . 59

3.35 Darstellung des Instabilitätsmaßes $\kappa$ für die verschiedenen Halterungskonturen (Simulationsergebnisse vgl. Tabelle 3.1, vgl. Abbildung 3.17 . . . . . 60

3.36 Vergleich der Zielfunktion für identische asymptotische Eigenfrequenzvorgaben mit ausgewählten Lösungen der Formoptimierungen (Simulationsergebnisse vgl. Tabelle 3.1, vgl. Abbildung 3.25 . . . . . . . . . . . . . 61

3.37 Darstellung der axialen Dehnungsmessung für unterschiedliche Messstrecken $l_{\mathrm{M}}$ (Simulationsergebnisse vgl. Tabelle 3.1, vgl. Abbildung 3.13) . . . . 62

3.38 Vergleich der verschiedenen Messstrategien (Simulationsergebnisse vgl. Tabelle 3.1. vgl. Abbildung 3.13, die letzten beiden Kurven fallen zusammen)

3.39 Auswertung der axialen Dehnungen in radialer Richtung für den Querschnitt in der Symmetrieebene (Simulationsergebnisse vgl. Tabelle 3.1) . . . 65

3.40 Darstellung des relativen Messfehlers $f$ bei Messung außerhalb der Symmetrieebene mit der neuen Messstrategie (Simulationsergebnisse vgl. Tabelle

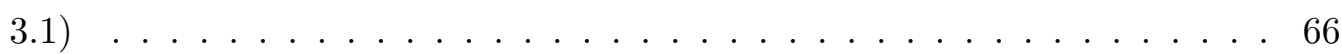

3.41 Überprüfung der neuen Messstrategie bei Variation des Kompressionsmoduls und der Reibung (Simulationsergebnisse vgl. Tabelle 3.1, die farbigen Kurven überlagern sich) . . . . . . . . . . . . . . . 67 67

3.42 Darstellung des relativen Messfehlers $f$ bei Variation der Reibung und des Kompressionsmoduls (Simulationsergebnisse vgl. Tabelle 3.1 . . . . . . 6 67

3.43 Darstellung des relativen Messfehlers $f$ für die neue Messstrategie bei unrundem Querschnitt (Simulationsergebnisse vgl. Tabelle 3.1 . . . . . . . . 69

3.44 Versuchsaufbau des Probekörper-Setups mit Messsystem . . . . . . . . . . 70

3.45 Zusammenbau des Probekörper-Setups . . . . . . . . . . . . . . 71 
3.46 Versuchsaufbau mit und ohne Ausrichteinheit . . . . . . . . . . . . . . 71

3.47 Gegenüberstellung: grafischer Ablaufeditor von Zwick/Roell und Erweiterung mit der externen Sollwertsteuerung. Die Abbildung ist in leicht modifizierter Form Kanzenbach u.a. (2016a) entnommen. Wiederverwendung mit Genehmigung. (c) 2016 Elsevier Ltd. . . . . . . . . . . . . . . . . 72

3.48 Blockdiagramm zur Realisierung der externen Sollwertsteuerung. Die Abbildung ist in leicht modifizierter Form Kanzenbach u. a. (2016a) entnommen. Wiederverwendung mit Genehmigung. (c) 2016 Elsevier Ltd. . . . . . . . . . 73

3.49 Hantelprobekörper (gefüllter EPDM) mit unterschiedlicher Beschichtung in Vorbereitung für die Messung mittels DIC (Abbildung in Anlehnung an die Arbeit von Oelsch (2017) durchgeführt an der Professur Festkörpermechanik, TU Chemnitz) . . . . . . . . . . . . . . . . . . 744

3.50 Stauchversuch ohne Halterungskonturen (Messung für gefüllten EPDM), Messsystem DIC. Quelle der Gesamtabbildung: Kanzenbach u. a. (2019a). Wiederverwendung mit Genehmigung. (c) 2019 Elsevier Ltd. . . . . . . . . 75

3.51 Stauchversuch des Hantelprobekörpers (gefüllter EPDM) mit Halterungskonturen. Die Abbildung ist in leicht modifizierter Form Kanzenbach u. a. (2018) entnommen. Wiederverwendung mit Genehmigung. () 2018 Elsevier Ltd . . . . . . . . . . . . . . . . . . . . 76

3.52 Darstellung des Mittelpunktversatzes des Hantelprobekörpers (Messung für gefüllten $\mathrm{EPDM}) \ldots \ldots \ldots \ldots \ldots \ldots 77$

3.53 Versuchsaufbau zur Untersuchung des Reibeinflusses . . . . . . . . . . 78

3.54 Darstellung des dynamischen Moduls über der Frequenz zur Untersuchung des Einflusses der Reibung (Messungen für gefüllten EPDM) . . . . . . . 797

3.55 Vergleich der Halterungskonturen bei einer Stauchung von ca. 50 \% (Messungen für gefüllten EPDM), Messsystem DIC . . . . . . . . . . . 80

3.56 Darstellung des Mittelpunktversatzes für die verschiedenen Halterungskonturen (Messungen für gefüllten EPDM) . . . . . . . . . . . . 81

3.57 Mehrstufiger Zugversuch (Messungen für gefüllten EPDM), Messsystem MultiXtens (Die Kurven schwarz und blau fallen zusammen) . . . . . . 82

3.58 Mehrstufiger Zugversuch (Messungen für gefüllten NR), Messsystem MultiXtens (Die Kurven schwarz und blau fallen zusammen). Die Abbildung ist in leicht modifizierter Form Kanzenbach u. a. (2016b) entnommen. Wiederverwendung mit Genehmigung. @ 2016 Wiley-VCH Verlag GmbH \& Co. KGaA, Weinheim . . . . . . . . . . . . . . . 83

3.59 Vergleich von Druck-Zug (schwarz) und Zug-Druckversuchen (rot) (Messungen für gefüllten EPDM), Messsystem DIC. Die Abbildung ist in leicht modifizierter Form Kanzenbach u. a. (2018) entnommen. Wiederverwendung mit Genehmigung. (C) 2018 Elsevier Ltd. . . . . . . . . . . . . . . . . 84 
3.60 Vergleich von Druck-Zug (schwarz) und Zug-Druckversuchen (rot) (Messungen für gefüllten NR), Messsystem DIC. Die Abbildung ist in leicht modifizierter Form Kanzenbach u.a. (2018) entnommen. Wiederverwendung mit Genehmigung. (c) 2018 Elsevier Ltd. . . . . . . . . . . . . . . . . . 884

3.61 Multi-Hystereseversuche (Messungen für gefüllten NR), Messsystem DIC (Messungen aus der Arbeit von Oelsch (2017) durchgeführt an der Professur Festkörpermechanik, TU Chemnitz) . . . . . . . . . . . 86

3.62 Darstellung der extremen Stauchung (Messungen für gefüllten EPDM), ermittelt über die Messung der Umfangsstreckung via DIC . . . . . . . . . . . 87

3.63 Darstellung der extremen Stauchung (Messungen für gefüllten NR), ermittelt über die Messung der Umfangsstreckung via DIC . . . . . . . . . . . . . 87

3.64 Entwicklung der lokalen Faltenbildung bei extremen Stauchungen für gefüllten $\mathrm{NR} \ldots \ldots \ldots \ldots \ldots \ldots \ldots \ldots \ldots$

4.1 Darstellung der Hauptinvarianten $I_{1}$ und $I_{2}$ bei verschiedenen Beanspruchungsarten (vgl. Krawietz 1986) . . . . . . . . . . . . . . 90

4.2 Darstellung der einfachen Scherung unter Annahme idealer Inkompressibilität 92

4.3 Darstellung des lokalen Fehlermaßes $\eta_{\mathrm{s}}$ für den idealisierten Scherprobekörper mit den Abmaßen: $10 \times 100 \times 2$ bei einem Schermaß von $s=1 \ldots 95$

4.4 Spannungs-Schermaßdiagramm für den idealisierten Scherprobekörper (Simulationsergebnisse vgl. Tabelle 4.1 . . . . . . . . . . . . . 95

4.5 Globaler Messfehler $\eta_{\mathrm{T}}$ für den idealisierten Scherprobekörper (Simulationsergebnis vgl. Tabelle 4.1 . . . . . . . . . . . . . . . . . 96

4.6 Mittelwert $\bar{\sigma}_{\text {eqv }}$ für den idealisierten Scherprobekörper (Simulationsergebnisse vgl. Tabelle 4.1 . . . . . . . . . . . . . . . . . . 979

4.7 Inhomogenitätsmaß $\xi_{\mathrm{s}}$ für den idealisierten Scherprobekörper für unterschiedliche Auswertebereiche (Simulationsergebnisse vgl. Tabelle 4.1) . . . . 98

4.8 Beuluntersuchungen für den idealisierten Scherprobekörper unter Variation der Dicke $t_{0} \in[2,4] \mathrm{mm}$ bei einer Winkelimperfektion von $\varphi=5^{\circ}$ (Simulationsergebnisse vgl. Tabelle 4.1 . . . . . . . . . . . . . . 999

4.9 Darstellung des lokalen Fehlermaßes $\eta_{\mathrm{s}}$ für den Akzo Nobel Scherprobekörper mit den Abmaßen: $10 \times 100 \times 2$ bei einer Scherbelastung von $u_{x}=$

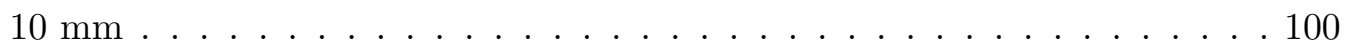

4.10 Spannungs-Schermaßdiagramm für den Akzo Nobel Scherprobekörper (Simulationsergebnisse vgl. Tabelle 4.1, vgl. Abbildung 4.4 . . . . . . . . . 101

4.11 Globaler Messfehler $\eta_{\mathrm{T}}$ für den Akzo Nobel Scherprobekörper (Simulationsergebnisse vgl. Tabelle 4.1, vgl. Abbildung 4.5 . . . . . . . . . . . . 101

4.12 Darstellung des lokalen Fehlermaßes $\eta_{\mathrm{s}}$ an einem repräsentativen Materialstreifen mit fixierten Stirnflächen bei einem Schermaß von $s=1 \ldots$. . . . 102 
4.13 Prinzipskizze eines Scherprobekörpers mit formschlüssiger Verbindung mittels Stiften (Grundgeometrie in Anlehnung an den Scherprobekörper der Firma Akzo Nobel, siehe Ihlemann 2003) . . . . . . . . . . . . . . . 104

4.14 Darstellung des lokalen Fehlermaßes $\eta_{\mathrm{s}}$ für einen repräsentativen Materialstreifen (Pin-Durchmesser $d=0.5 \mathrm{~mm}$ ) bei einem Schermaß von $s=1 \ldots 104$

4.15 Darstellung des globalen Messfehlers $\eta_{\mathrm{T}}$ für verschiedene flächige Scherprobekörper (Simulationsergebnisse vgl. Tabelle 4.1, vgl. Abbildung 4.11 . . . 105

4.16 Darstellung des Inhomogenitätsmaßes $\xi_{\mathrm{s}}$ für verschiedene flächige Scherprobekörper (Simulationsergebnisse vgl. Tabelle 4.1, vgl. Abbildung 4.7) . . . . 106

4.17 Versuchsaufbau der Schervorrichtung mit Messsystem . . . . . . . . . . . 107

4.18 Lochung der Elastomermatte mittels Stanzmatrix . . . . . . . . . . . . 108

4.19 Symmetrischer Scherversuch mit der neuen Schervorrichtung (Messungen

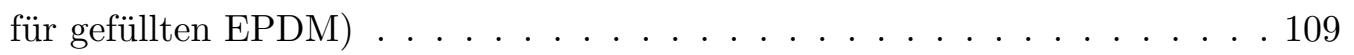

4.20 Lokale Darstellung des Schermaßes $s$ für verschiedene Laststufen . . . . . . 110

4.21 Spannungs-Schermaßdiagramm für verschiedene Mattendicken (Messungen

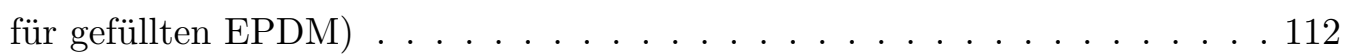

4.22 Kappa-Schermaßdiagramm für verschiedene Mattendicken (Simulationsergebnisse vgl. Tabelle 4.1 und Messungen für gefüllten EPDM, vgl. Abbildung 4.8 . $\ldots \ldots \ldots$

5.1 Parameteranpassung des Rabkin-Modells an die homogenen Zug-/Druckversuche (Messungen für gefüllten EPDM, Anpassung von M. Sc. Gelke an der Professur Festkörpermechanik, TU Chemnitz) . . . . . . . . . . . . . 116

5.2 Vergleich der Probekörper und der homogenen Simulation (Messung für gefüllten EPDM, vgl. Abbildung 5.1 . . . . . . . . . . . . . . 117

5.3 Vergleich des globalen Kraft-Wegverhaltens (Traversenweg) zwischen Messung für gefüllten EPDM und Simulation . . . . . . . . . . . . . . 118

5.4 Vergleich zwischen Scherversuch für gefüllten EPDM (vgl. Abbildung 4.19) und homogener Simulation (Anpassung erfolgte an Zug-/Druckkurven) . . . 119

5.5 Darstellung der Sinusverläufe mit unterschiedlichen Potenzen zur Untersuchung des ratenabhängigen Materialverhaltens (Simulationsergebnisse) . . . 121

5.6 Spannungs-Dehnungsantwort der Sinusverläufe mit unterschiedlichen Potenzen für den Zug-/Druckprobekörper (Messungen für gefüllten EPDM) . 121

5.7 Spannungs-Schermaßantwort der Sinusverläufe mit unterschiedlichen Potenzen für den Scherprobekörper (Messungen für gefüllten EPDM) . . . . . 122

5.8 Darstellung der verzerrten Sinusverläufe (Sägezahnverlauf) zur Untersuchung des ratenabhängigen Materialverhaltens (Simulationsergebnisse) . . . 123 
5.9 Spannungs-Dehnungsantwort der verzerrten Sinusverläufe (Sägezahnverlauf) für den Zug-/Druckprobekörper (Messungen für gefüllten EPDM) . . . . . . 124

5.10 Darstellung der gestreckten Cosinusverläufe (Kettenradverlauf) zur Untersuchung des ratenabhängigen Materialverhaltens (Simulationsergebnisse) . . 125

5.11 Spannungs-Dehnungsantwort der gestreckten Cosinusverläufe (Kettenradverlauf) für den Zug-/Druckprobekörper (Messungen für gefüllten EPDM) . 125

5.12 Automatische Einspannvorrichtung zur Implementierung des ProbekörperSetups in den Industriealltag (Abbildung von Dr. Folke J. Tölle aus der Firma Vibracoustic GmbH) . . . . . . . . . . . . . . . . . . . . 127

5.13 Versuchsaufbau des Probekörper-Setups an einer elektromechanischen Prüfmaschine mit integriertem KEYENCE-Messsystem: (oben) Zugversuch, (unten) Druckversuch . . . . . . . . . . . . . . . . . . 128

5.14 Versuchsaufbau des Probekörper-Setups an einer servo-hydraulischen Prüfmaschine mit integriertem KEYENCE-Messsystem . . . . . . . . . . . . . 129

A.1 Darstellung der Zielfunktion $f$ für identische asymptotische Eigenfrequenzvorgaben $l_{0}=50 \mathrm{~mm}$, Schnittpunkt $\varepsilon=-0.4$ (Simulationsergebnis) . . . 135

A.2 Darstellung der Zielfunktion $f$ für identische asymptotische Eigenfrequenzvorgaben $l_{0}=55 \mathrm{~mm}$, Schnittpunkt $\varepsilon=-0.3$ (Simulationsergebnis) . . . 135

A.3 Darstellung der $z$-Verschiebung für den idealisierten Scherprobekörper mit den Abmaßen: $10 \times 100 \times 2$ bei einer Winkelimperfektion von $\varphi=2.5^{\circ} \ldots 136$

A.4 Darstellung des lokalen Fehlermaßes $\eta_{\mathrm{s}}$ für den neuen Scherprobekörper mit den Abmaßen: $10 \times 100 \times 2$ mit Pin-Durchmesser $d=0.5 \mathrm{~mm}$ bei einer Scherbelastung von $u_{x}=10 \mathrm{~mm}$ (Symmetrie in negativer $z$-Richtung) . . . 136

A.5 Spannungs-Schermaßantwort des verzerrten Sinusverlaufes (Sägezahnverlauf) für den einfachen Scherversuch (Messungen für gefüllten EPDM) . . . 137

A.6 Miniaturisierung des Probekörper-Setups für homogene Alterungsuntersuchungen (Simulationsergebnisse) . . . . . . . . . . . . 137 



\section{Einleitung}

\subsection{Motivation der Arbeit}

Trotz modernster Simulationswerkzeuge, wie die Finite-Elemente-Methode (FEM), hat der experimentelle Versuch nichts an seiner Bedeutung und Relevanz verloren. Ausgehend von komplexen Materialverhalten, wie es technische Elastomere zeigen, werden verschiedene Probekörper benötigt, um die grundlegenden Eigenschaften untersuchen zu können. Da sowohl für die phänomenologische Charakterisierung als auch für die Parameteridentifikation von Stoffgesetzen Messdaten, die aus homogenen Versuchen resultieren, benötigt werden, entstehen hohe Anforderungen bezüglich der Probekörperentwicklung.

Eine besonders wichtige Grundbeanspruchungsart für viele technische Anwendungsfälle ist der einachsige Zug/Druck. Gerade der Zugversuch kann dabei als einer der bekanntesten Standardversuche angesehen werden (vgl. Brown 2006). Demgegenüber ist der einachsige homogene Druckversuch mit großen Herausforderungen verbunden. In der Abbildung 1.1 ist dazu exemplarisch die Geometrie eines Hantelprobekörpers dargestellt, wie er zur Untersuchung kombinierter einachsiger Zug-/Druckversuche verwendet wird. Die Dehnungsmessung erfolgt dabei beispielsweise optisch mittels Laserextensometer, welches den Abstand zweier reflektierender Punkte bestimmt. In der Mitte der Abbildung ist
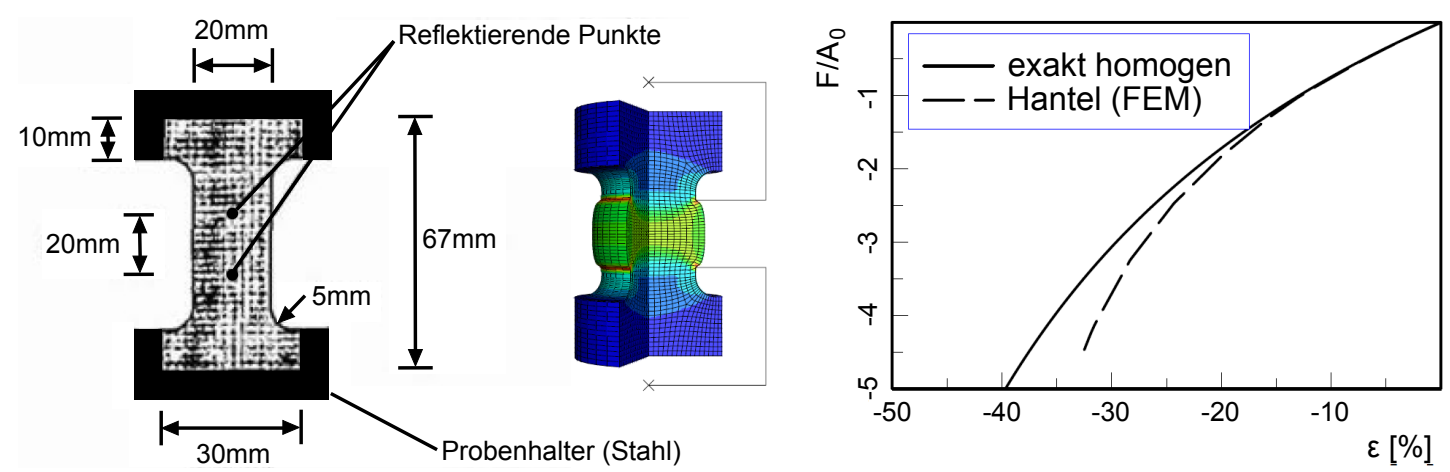

Abb. 1.1 - Zug-/Druckprobekörper (links) Probengeometrie (Abbildung aus der Arbeit von Lion 2000, S. 73) und (rechts) Fe-Simulation mit Auswertung (Abbildungen aus der Veröffentlichung von Alshuth u. a. 2007)

das Ergebnis einer FE-Simulation mit dem Hantelprobekörper und zugehöriger Halterung dargestellt (vgl. Alshuth u. a. 2007). Aus dem Diagramm (Abbildung 1.1 rechts) wird deutlich, dass der Probekörper schon bei Stauchungen größer 30 \% zu großen Abweichungen gegenüber der homogenen Lösung führt. Hinzu kommt, dass bei Druckversuchen mit 
unerwünschten Effekten wie Knicken zu rechnen ist. All diese Aspekte lassen erahnen, wie schwierig der homogene Druckversuch zu realisieren ist.

Einer der Hauptschwerpunkte dieser Arbeit besteht in der Entwicklung eines neuen verbesserten Probekörper-Setups (bestehend aus Probekörper und Halterung) zur Realisierung hochpräziser einachsiger Zug-/Druckversuche. Dabei soll sowohl die Homogenität im Messbereich als auch die maximal zu erreichenden Stauchungen wesentlich verbessert werden. Erreicht wird diese herausragende Eigenschaftskombination durch ein spezielles Design der Halterungskontur. Der Probekörper selbst soll dabei seine vergleichsweise einfache Hantelform beibehalten und lediglich einen verlängerten Mittelteil aufweisen. Die Geometrie der Halterung wird dagegen auf Basis spezieller Dynamik-FEM-Simulationen ermittelt, welche eine quantifizierbare und über den Stauchprozess genau zu erfassende Größe darstellt. Damit können nun erstmals hochpräzise Stauchungen bis 70 \% realisiert und gemessen werden, was ganz neue Möglichkeiten im Bereich der Materialcharakterisierung eröffnet.

Neben dem einachsigen Zug/Druck stellt die einfache Scherung eine weitere elementare Grundbeanspruchungsart dar. Beispielsweise erfahren wichtige Funktionselemente aus Gummi wie Fahrwerksbuchsen oder Erdbebendämpfer in hohem Maße Scherdeformationen. Die exakte Charakterisierung eines homogen gescherten Materialbereiches ist also von größtem Interesse für die Bestimmung wichtiger Materialparameter, insbesondere bei dynamischen Untersuchungen. Der dabei am häufigsten zur Anwendung kommende Scherprobekörper ist in Form eines doppelt zylindrischen Designs konzipiert (vgl. Abbildung 2.3. links). Eines der großen Nachteile dieses Scherprobekörpers liegt in der Problematik, dass er an den Deckflächen angeklebt oder anvulkanisiert wird. Diese stoffschlüssige Verbindung hat den entscheidenden Nachteil, dass sie zu Materialirritationen in den Grenzbereichen, sowohl als auch zu Schrumpfverhalten innerhalb des Messbereiches (beispielsweise beim Anvulkanisieren) führt. Hinzu kommt, dass der Probekörper aufgrund seiner kompakten Struktur in keiner Weise für Alterungsuntersuchungen geeignet ist. Aus diesem Grund soll im Rahmen dieser Arbeit auch ein verbesserter Scherprobekörper zur Realisierung präziser Schermessungen entwickelt werden. Das Design soll dabei auf einem flächigen Probekörper beruhen. Diese Grundgeometrie hat dabei eine Reihe ganz wesentlicher Vorteile. Zum einen können Matten aus der Serienproduktion verwendet werden, zum anderen lassen sich Alterungsuntersuchungen, Untersuchungen mit faserverstärkten Materialien und Versuche mit Vorreckungen realisieren. Demgegenüber besteht eines der Grundprobleme von flächigen Scherprobekörpern darin, dass sich eine gleichmäßige Lasteinleitung vergleichsweise schwierig realisieren lässt. Aus diesem Grund soll diesbezüglich ein geeigneter Klemmbzw. Spannmechanismus entwickelt werden, der es ermöglicht Elastomermatten präzise zu scheren. Die dazu entwickelte Schervorrichtung wird getestet und zur Untersuchung grundlegender Eigenschaften technischer Elastomere eingesetzt. 


\subsection{Zielsetzung und Einordnung der eigenen Arbeit}

Das angestrebte Ziel dieser Arbeit besteht in der Weiterentwicklung bestehender Probekörperkonzepte. Ein Schwerpunkt soll dabei die grundlegende Auseinandersetzung mit der Problematik von Zug/Druck sowie Scherung als Hauptbeanspruchungsarten sein. Aufbauend auf diesen Erkenntnissen sollen neue verbesserte Probekörperkonzepte entwickelt werden. Dabei sind ganz unterschiedliche Zielsetzungen zu berücksichtigen. Zum einen sollen die Probekörper in ihrer Homogenität, zum anderen in den maximal zu erreichenden Dehnungen bzw. Stauchungen deutlich verbessert werden. Außerdem spielen Aspekte wie Handhabbarkeit und Wirtschaftlichkeit eine ebenso wichtige Rolle wie die universelle Einsetzbarkeit für Materialcharakterisierungen und die Identifikation von Materialparametern.

In Kapitel 2 werden zunächst die wichtigsten kontinuumsmechanischen Grundlagen, die zum Verständnis dieser Arbeit beitragen erläutert. Ein besonderer Schwerpunkt liegt dabei auf der Einführung hyperelastischer Stoffgesetze mit quasi-inkompressibler Formulierung, welche zur Definition von Fehlermaßen verwendet werden. Weiterhin gibt das Kapitel eine Übersicht über bisherige Prüfkörper, wie sie zur phänomenologischen Charakterisierung technischer Elastomere eingesetzt werden. Diesbezüglich werden in besonderer Weise bisherige Zug-/Druck und Scherprobekörper näher beleuchtet.

In Kapitel 3 erfolgt dann die experimentell-numerische Weiterentwicklung eines hochpräzisen Zug-/Druckprobekörpers. Zu Beginn des Kapitels werden theoretische Grundlagen zur Untersuchung der Knickstabilität von Stäben und Balken erarbeitet. Mit Hilfe der Finite-Elemente-Methode lassen sich dabei zum einen die analytischen Lösungen überprüfen, zum anderen können die verwendeten Verfahren für beliebige Strukturen erweitert werden. Eine besonders geeignete Größe zur Beschreibung des Stabilitätsverhaltens stellt dabei die erste Eigenfrequenz dar. In der Arbeit von Naumann (2010) wird ein Algorithmus zur Berechnung von Halterungskonturen vorgestellt, der es ermöglicht über die Vorgabe von Eigenfrequenzverläufen Halterungsgeometrien zu berechnen. Darauf aufbauend erfolgt die Optimierung der Halterungskontur mittels eingeführter Fehler- und Instabilitätsmaße. Der letzte große Abschnitt beschäftigt sich mit der experimentellen Validierung des neuen Probekörper-Setups. Dabei wird in besonderer Weise auf den Versuchsaufbau, die Messmethodik und einer erweiterten Prüfmaschinensteuerung eingegangen. Mit Hilfe der neuen Messstrategie, die ebenfalls in diesem Kapitel eingeführt wird, kann gezeigt werden, dass sich extreme Stauchungen bis $70 \%$ realisieren und messen lassen. Nach Abschluss verschiedener Qualitätstests erfolgt die Untersuchung phänomenologischer Eigenschaften technischer Elastomere, vorzugsweise bei großen Stauchungen.

Das Kapitel 4 beschäftigt sich mit der numerischen Entwicklung eines präzisen Scherprobekörpers. Im Gegensatz zu herkömmlichen Scherprobekörpern soll dieser weder geklebt 
noch anvulkanisiert werden. Mittels eingeführter Fehler- und Inhomogenitätsmaße werden verschiedene Einspanndesigns getestet und validiert. Dabei wird ersichtlich, dass besonders der Einfluss der Randbedingungen in Dickenrichtung einen erheblichen Einfluss auf die zu übertragende Scherdeformation ausübt. Mit einem speziellen Fixierdesign lassen sich Gummimatten unterschiedlicher Stärken einspannen und anschließend präzise scheren. Nach Abschluss der numerischen Entwicklung erfolgt die experimentelle Realisierung und die Durchführung präziser Schermessungen. Mit diesen Ergebnissen wird eine erste Validierung mit Simulationsdaten vorgenommen.

In Kapitel 5 werden die entwickelten Probekörperkonzepte für spezielle Anwendungsbeispiele eingesetzt. Neben der Identifikation von Parametern eines viskoelastoplastischen Stoffgesetzes bei der auf die homogenen Zug-/Druckversuche zurückgegriffen wird, soll eine Probekörpersimulation mit dem Stoffgesetz durchgeführt werden, um das hohe Potenzial des Probekörpers aufzeigen zu können. Weiterhin wird mit Hilfe der entwickelten hochpräzisen Probekörper und einer erweiterten Prüfmaschinensteuerung das ratenabhängige Materialverhalten von technischen Elastomeren untersucht. Damit wird insbesondere für die Entwicklung neuer Materialmodelle ein Zugang eröffnet, in dem die spezielle Phänomenologie technischer Elastomere sehr präzise untersucht werden kann. Zum Abschluss des Kapitels erfolgt die Implementierung des entwickelten Probekörper-Setups in den Industriealltag.

In Kapitel 6 werden die wichtigsten Ergebnisse dieser Arbeit zusammengefasst. Dabei kann in besonderer Weise dargelegt werden, dass sich nun erstmalig extreme Stauchungen bis einschließlich $70 \%$ realisieren lassen. Demgegenüber zeigt auch der entwickelte Scherprobekörper eine Vielzahl interessanter Anwendungsmöglichkeiten. Mit einem Ausblick hinsichtlich Lebensdaueruntersuchungen für das neu entwickelte Probekörper-Setup, vorzugsweise im Druckbereich, endet die Ausgestaltung dieser Arbeit. 


\section{Grundlagen}

\subsection{Grundbegriffe der Kontinuumsmechanik}

Zum Verständnis dieser Arbeit sollen im Folgenden die wichtigsten kontinuumsmechanischen Grundlagen erläutert werden. Dabei wird auf grundlegende Größen und Definitionen der Kontinuumsmechanik eingegangen. Die nachfolgenden Abschnitte erheben hierbei keinen Anspruch auf Vollständigkeit. Für weiterführende Ergänzungen wird auf die Arbeiten von Bergström (1999), Holzapfel (2000), Lion (2000), Haupt (2002) und Ihlemann (2003 $\& 2014)$ verwiesen.

\subsubsection{Tensoralgebra}

Die im Rahmen dieser Arbeit benötigten Vektoren und Tensoren werden durch Unterstriche pro Stufe gekennzeichnet. Beispielsweise stellt der Tensor $\underline{\underline{X}}$ einen Tensor 2. Stufe dar. Dieser setzt sich aus dem Koeffizient $X_{a b}$ und der dyadischen Verknüpfung der Basisvektoren $\underline{e}_{a}$ und $\underline{e}_{b}$ zusammen. Die Indizes $a$ und $b$ kennzeichnen dabei ein kartesisches Basisvektorsystem. Nach der Einstein'schen Summationskonvention wird dabei über doppelt auftretende Indizes summiert:

$$
\underline{\underline{X}}=\sum_{a=x}^{z} \sum_{b=x}^{z} X_{a b} \underline{e}_{a} \circ \underline{e}_{b}=X_{a b} \underline{e}_{a} \circ \underline{e}_{b} \quad \text { mit: } \quad a, b=x, y, z
$$

Die Koeffizienten eines Tensors 2. Stufe $\left[X_{a b}\right]$ werden, wenn nicht anders angegeben, in einer 3x3-Matrix angeordnet:

$$
\left[X_{a b}\right]=\left[\begin{array}{ccc}
X_{x x} & X_{x y} & X_{x z} \\
X_{y x} & X_{y y} & X_{y z} \\
X_{z x} & X_{z y} & X_{z z}
\end{array}\right]
$$

Um Tensoren miteinander verrechnen zu können, wird zunächst das Kronecker-Symbol $\delta_{a b}$ als Punktprodukt zweier Basisvektoren desselben Systems eingeführt. Mit dessen Hilfe lassen sich auch die Koeffizienten eines zweistufigen Einheitstensors $\underline{\underline{I}}$ (auch Metriktensor 
genannt) beschreiben.

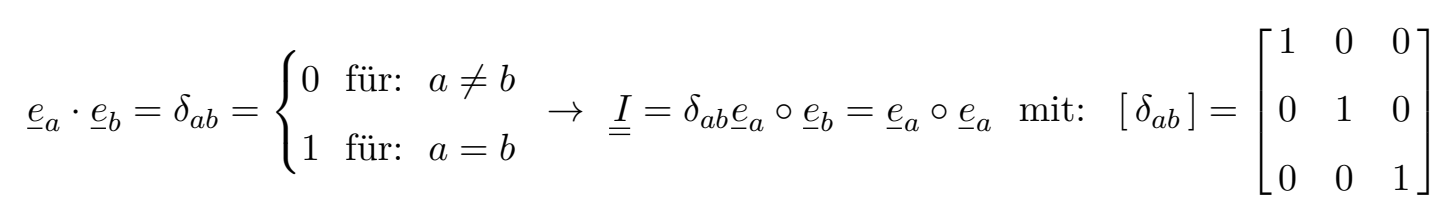

Im Folgenden werden die verschiedenen Rechenregeln von Tensoren eingeführt. Bei der Addition bzw. Subtraktion von Tensoren werden lediglich die Koeffizienten miteinander verrechnet. Führt man hingegen das einfache Punktprodukt zwischen zwei Tensoren 2. Stufe aus, so entsteht wiederum ein Tensor der 2. Stufe. Im Vergleich dazu ergibt sich beim doppelten Punktprodukt zweier Tensoren 2. Stufe eine skalare Größe. Verwendet man hingegen das dyadische Produkt, so werden die Basisvektoren nicht miteinander verrechnet, sondern bleiben in ihrer Reihenfolge unverändert stehen. Es ergibt sich hier ein Tensor der 4. Stufe.

$$
\begin{aligned}
& \underline{\underline{X}} \pm \underline{\underline{Y}}=X_{a b} \underline{e}_{a} \circ \underline{e}_{b} \pm Y_{a b} \underline{e}_{a} \circ \underline{e}_{b}=\left(X_{a b} \pm Y_{a b}\right) \underline{e}_{a} \circ \underline{e}_{b} \\
& \underline{\underline{X}} \cdot \underline{\underline{Y}}=X_{a b} \underline{e}_{a} \circ \underline{e}_{b} \cdot Y_{c d} \underline{e}_{c} \circ \underline{e}_{d}=X_{a b} Y_{b d} \underline{e}_{a} \circ \underline{e}_{d} \\
& \underline{\underline{X}} \cdot \underline{\underline{Y}}=X_{a b} \underline{e}_{a} \circ \underline{e}_{b} \cdot Y_{c d} \underline{e}_{c} \circ \underline{e}_{d}=X_{a b} Y_{b a} \\
& \underline{\underline{X}} \circ \underline{\underline{Y}}=X_{a b} \underline{e}_{a} \circ \underline{e}_{b} \circ Y_{c d} \underline{e}_{c} \circ \underline{e}_{d}=X_{a b} Y_{c d} \underline{e}_{a} \circ \underline{e}_{b} \circ \underline{e}_{c} \circ \underline{e}_{d}
\end{aligned}
$$

Da in der Literatur häufig in Verbindung mit dem doppelten Punktprodukt die Operation „::" verwendet wird, soll der Zusammenhang zu der hier verwendeten Operation „.." hergestellt werden:

$$
\underline{\underline{X}}: \underline{\underline{Y}}=X_{a b} Y_{a b} \Rightarrow \underline{\underline{X}} \cdot \underline{\underline{Y}}=\underline{\underline{X}}: \underline{\underline{Y}}^{T}
$$

Um weiterhin besondere Eigenschaften von Tensoren genauer charakterisieren zu können, sollen im Folgenden einige Anteile näher betrachtet werden. Eine spezielle Form stellt dabei der symmetrische Tensor dar. Dieser hat als Tensor zweiter Stufe nur sechs voneinander unabhängige Koeffizienten. Im Vergleich dazu besitzt ein unsymmetrischer Tensor neun unabhängige Koeffizienten (vgl. Gleichung (2-2)). Dieser unsymmetrische Tensor $\underline{\underline{X}}$ kann stets in einen symmetrischen Anteil $\stackrel{\underline{X}}{\underline{S}}$ und einen antisymmetrischen Anteil $\stackrel{A}{\underline{\underline{X}}}$ zerlegt werden.

$$
\underline{\underline{X}}=\underline{\underline{X}}+\stackrel{A}{\underline{X}} \quad \text { mit: }\left\{\begin{array}{l}
\underline{\underline{S}}=\frac{1}{2}\left(\underline{\underline{X}}+\underline{\underline{X}}^{T}\right) \\
\stackrel{\underline{A}}{\underline{X}}=\frac{1}{2}\left(\underline{\underline{X}}-\underline{\underline{X}}^{T}\right)
\end{array}\right.
$$


Der Tensor $\underline{\underline{X}}^{T}$ beschreibt dabei einen transponierten Tensor 2. Stufe, bei dem lediglich die Basisvektoren vertauscht werden.

$$
\underline{\underline{X}}^{T}=\underline{e}_{a} \cdot \underline{\underline{X}} \circ \underline{e}_{a}=\underline{e}_{a} \cdot X_{c b} \underline{e}_{c} \circ \underline{e}_{b} \circ \underline{e}_{a}=X_{a b} \underline{e}_{b} \circ \underline{e}_{a}
$$

Ein weiterer wichtiger Operator für Tensoren zweiter Stufe stellt die Invertierung eines Tensors $\underline{\underline{X}}^{-1}$ dar. Im einfachen Punktprodukt mit dem Tensor $\underline{\underline{X}}$ ergibt sich dabei wieder der Einheitstensor $\underline{\underline{I}}$ (vgl. Gleichung (2-3)). Dessen Koeffizienten verhalten sich invariant, sind also identisch bei allen Darstellungen in orthonormierten Basissystemen.

$$
\underline{\underline{X}} \cdot \underline{\underline{X}}^{-1} \stackrel{!}{=} \underline{\underline{I}}
$$

Neben der symmetrischen und antisymmetrischen Zerlegung lässt sich ein Tensor auch in einen hydrostatischen Anteil $\stackrel{\underline{\underline{X}}}{\underline{u}}$ und einen deviatorischen Anteil $\underline{\underline{X}}^{\prime}$ zerlegen.

$$
\underline{\underline{X}}=\underline{\underline{\underline{X}}}+\underline{\underline{X}}^{\prime} \quad \text { mit: }\left\{\begin{array}{l}
\underline{\underline{X}}=\frac{1}{3}(\underline{\underline{X}} \cdot \underline{\underline{I}}) \underline{\underline{I}} \\
\underline{\underline{X}}^{\prime}=\underline{\underline{X}}-\frac{1}{3}(\underline{\underline{X}} \cdot \underline{\underline{I}}) \underline{\underline{I}}
\end{array}\right.
$$

Im Weiteren lassen sich aus einem Tensor $\underline{\underline{X}}$ Invarianten berechnen. Die etablierten Hauptinvarianten $I_{1}, I_{2}$ und $I_{3}$ sind wie folgt definiert:

$$
\begin{aligned}
& I_{1}(\underline{\underline{X}})=\underline{\underline{X}} \cdot \underline{\underline{I}}=\operatorname{spur}\left[X_{a b}\right] \\
& I_{2}(\underline{\underline{X}})=\frac{1}{2}\left[I_{1}(\underline{\underline{X}})^{2}-I_{1}\left(\underline{\underline{X}}^{2}\right)\right] \\
& I_{3}(\underline{\underline{X}})=I_{1}(\underline{\underline{X}}) I_{2}(\underline{\underline{X}})-\frac{1}{3}\left[I_{1}(\underline{\underline{X}})^{3}-I_{1}\left(\underline{\underline{X}}^{3}\right)\right]=\operatorname{det}\left[X_{a b}\right]
\end{aligned}
$$

Mittels der Hauptinvarianten können über das charakteristische Polynom die Eigenwerte $X_{A}$ eines Tensors $\underline{\underline{X}}$ berechnet werden:

$$
X_{A}^{3}-I_{1}(\underline{\underline{X}}) X_{A}^{2}+I_{2}(\underline{\underline{X}}) X_{A}-I_{3}(\underline{\underline{X}})=0 .
$$

Sind die drei Eigenwerte $X_{A}$ eines Tensors 2. Stufe von Null verschieden, so lässt sich der Tensor invertieren. Sind die Eigenwerte darüber hinaus noch reell, so liegt ein symmetrischer Tensor vor. Eine weitere Klasse von Invarianten stellen die Vergleichsspannungen, die aus einem allgemeinen dreiachsigen Spannungszustand (siehe nächster Abschnitt 2.1.2 einen skalaren Vergleichswert liefern, dar. Ein bekannter Vertreter ist dabei die Formänderungsenergiehypothese, die auch als Von-Mises-Vergleichsmaß bezeichnet wird:

$$
I_{\mathrm{VM}}(\underline{\underline{X}})=\sqrt{\frac{3}{2}\left(\underline{\underline{X}}^{\prime} \cdot \underline{\underline{X}}^{\prime T}\right)}=\sqrt{\frac{1}{2}\left[\left(X_{\mathrm{I}}-X_{\mathrm{II}}\right)^{2}+\left(X_{\mathrm{II}}-X_{\mathrm{III}}\right)^{2}+\left(X_{\mathrm{I}}-X_{\mathrm{III}}\right)^{2}\right]} .
$$


Eine weitere Invariante aus der Grundlagenmechanik ist das Vergleichsmaß nach Tresca:

$$
I_{\mathrm{VT}}(\underline{\underline{X}})=\max \left[\left|X_{\mathrm{I}}-X_{\mathrm{II}}\right|,\left|X_{\mathrm{II}}-X_{\mathrm{III}}\right|,\left|X_{\mathrm{I}}-X_{\mathrm{III}}\right|\right] .
$$

\subsubsection{Verzerrungs- und Spannungstensoren}

Um Materialdeformationen kontinuumsmechanisch beschreiben zu können, werden verschiedene tensorielle Verformungsgrößen benötigt. Eine der wichtigsten Ausgangsgrößen ist dabei der Deformationsgradient $\underline{\underline{F}}$, welcher die Verknüpfung zwischen Referenz- und aktueller Konfiguration eines Kontinuums beschreibt.

$$
\underline{\underline{F}}=\frac{\partial \underline{r}}{\partial \underline{\widetilde{r}}}=(\underline{\widetilde{\nabla}} \circ \underline{r})^{T}=\left(\underline{e}_{a} \circ \frac{\partial \underline{r}}{\partial \widetilde{x}_{a}}\right)^{T}=\frac{\partial x_{b}}{\partial \widetilde{x}_{a}} \underline{e}_{b} \circ \underline{e}_{a} \quad \text { mit: } \quad \underline{\widetilde{\nabla}}=\underline{e}_{a} \frac{\partial}{\partial \widetilde{x}_{a}}
$$

Über den Deformationsgradienten lassen sich im Weiteren die verschiedenen eulerschen und lagrangeschen Verzerrungsmaße berechnen. Die Bezeichnung Euler bzw. Lagrange ist dabei auf die Beobachterabhängigkeit der Verzerrungsmaße zurückzuführen. Beispielsweise stellen eulersche Größen beobachterunabhängige und lagrangesche Größen beobachterabhängige Tensoren dar (im Sinne von Ihlemann 2014). Dort findet sich zudem eine detailliertere Übersicht zur Beobachterabhängigkeit von Verzerrungsgrößen. Im Rahmen dieser Arbeit werden zunächst die wichtigsten eulerschen und lagrangeschen Verzerrungsmaße zusammengetragen. Die eulerschen Größen lauten dabei wie folgt:

$$
\begin{aligned}
\underline{\underline{V}} & =\sqrt{\underline{\underline{F} \cdot \underline{F}^{T}}} & & \text { Linker Strecktensor } \\
\underline{\underline{b}} & =\underline{\underline{F}} \cdot \underline{\underline{F}}^{T}=\underline{\underline{V}}^{2} & & \text { Linker Cauchy-Green-Tensor } \\
\underline{\underline{\varepsilon}} & =\frac{1}{2}\left(\underline{\underline{I}}-\underline{\underline{b}}^{-1}\right) & & \text { Almansischer Verzerrungstensor } \\
\underline{\underline{h}} & =\frac{1}{2} \ln ((\underline{\underline{b}}))=\ln ((\underline{\underline{V}})) & & \text { Eulerscher Hencky-Tensor }
\end{aligned}
$$

Demgegenüber sind die lagrangeschen Verzerrungsmaße wie folgt definiert:

$$
\begin{array}{rlrl}
\underline{\underline{U}} & =\sqrt{\underline{\underline{F}}^{T} \cdot \underline{\underline{F}}} & & \text { Rechter Strecktensor } \\
\underline{\underline{C}} & =\underline{\underline{F}}^{T} \cdot \underline{\underline{F}}=\underline{\underline{U}}^{2} & & \text { Rechter Cauchy-Green-Tensor } \\
\underline{\underline{\gamma}} & =\frac{1}{2}(\underline{\underline{C}}-\underline{\underline{I}}) & & \text { Greenscher Verzerrungstensor } \\
\underline{\underline{H}}=\frac{1}{2} \ln ((\underline{\underline{C}}))=\ln ((\underline{\underline{U}})) & \text { Lagrangescher Hencky-Tensor }
\end{array}
$$

Analog zu den Verzerrungsmaßen lassen sich auch Spannungstensoren eulerschen und lagrangeschen Beobachtern zuordnen. Dabei stellt der Cauchy-Spannungstensor $\underline{\underline{\sigma}}$ den Vertreter der eulerschen und der 2. Piola-Kirchhoff-Spannungstensor $\underline{\underline{T}}$ den Vertreter der lagrangeschen Betrachtungsweise dar. Der symmetrische Cauchy-Spannungstensor wird 
dabei über den Normalenvektor $\underline{n}$ und den Spannungsvektor $\underline{s}$ wie folgt eingeführt:

$$
\underline{s}=\underline{n} \cdot \underline{\underline{\sigma}} .
$$

Mit Hilfe der pull-back-Operation (vgl. Holzapfel 2000), welche einen Wechsel zwischen verschiedenen Konfigurationen ermöglicht, kann der ebenfalls symmetrische 2. Piola-Kirchhoff-Spannungstensor wie folgt angegeben werden:

$$
\underline{\underline{\underline{T}}}=\frac{\widetilde{\rho}}{\rho} \underline{\underline{F}}^{-1} \cdot \underline{\underline{\sigma}} \cdot \underline{\underline{F}}^{-T} .
$$

Ähnlich wie beim Deformationsgradienten, der keiner Betrachtungsweise zuzuordnen ist, gibt es noch eine Mischform: den 1. Piola-Kirchhoff-Spannungstensor $\underline{\underline{T}}$. Dieser in der Regel oft unsymmetrische Spannungstensor ist wie folgt definiert:

$$
\underline{\underline{T}}=\frac{\widetilde{\rho}}{\rho} \underline{F}^{-1} \cdot \underline{\underline{\sigma}} .
$$

Zusammenfassend können die verschiedenen Spannungsmaße über den Deformationsgradienten wie folgt umgerechnet werden:

$$
\underline{\underline{\sigma}}=\frac{\rho}{\widetilde{\rho}} \underline{\underline{F}} \cdot \underline{\underline{T}}=\frac{\rho}{\widetilde{\rho}} \underline{\underline{F}} \cdot \underline{\underline{\widetilde{T}}} \cdot \underline{\underline{F}}^{T} .
$$

Um auch Aussagen über zeitliche Änderungen von Verzerrungs- und Spannungstensoren treffen zu können, sollen im Folgenden Zeitableitungen näher betrachtet werden. Eine besondere Rolle nimmt dabei die substantielle oder auch materielle Zeitableitung ein. Diese beschreibt die Änderung eines materiellen Punktes über den Deformationsprozess:

$$
\underline{\underline{\underline{X}}}:=\lim _{\Delta t \rightarrow 0} \frac{\underline{\underline{X}}\left(x_{a}, t+\Delta t\right)-\underline{\underline{X}}\left(x_{a}, t-\Delta t\right)}{2 \Delta t} .
$$

Wird die substantielle Zeitableitung auf lagrangesche Tensoren angewendet, so entstehen stets lagrangesche Größen. Im Folgenden wird die lagrangesche Zeitableitung durch ein hochgestelltes „॰ " gekennzeichnet.

$$
\underline{\underline{\underline{X}}}=\dot{X}_{a b} \underline{e}_{a} \circ \underline{e}_{b}
$$

Um verschiedene eulersche Zeitableitungen angeben zu können, werden hingegen spezielle Berechnungsvorschriften benötigt (vgl. Ihlemann 2003 \& 2014). Eine in diesem Zusammenhang wichtige Größe stellt der Geschwindigkeitsgradient $\underline{\underline{L}}$ dar.

$$
\underline{\underline{L}}=(\underline{\nabla} \circ \underline{v})^{T}=\underline{\underline{F}} \cdot \underline{\underline{F}}^{-1}=\underline{\underline{D}}+\underline{\underline{W}} \quad \text { mit: }\left\{\begin{array}{l}
\underline{\underline{D}}=\frac{1}{2}\left(\underline{\underline{L}}+\underline{\underline{L}}^{T}\right) \\
\underline{\underline{W}}=\frac{1}{2}\left(\underline{\underline{L}}-\underline{\underline{L}}^{T}\right)
\end{array}\right.
$$


Hierbei wird der Tensor $\underline{\underline{D}}$ als Tensor der Formänderungsgeschwindigkeit und der Tensor $\underline{\underline{W}}$ als Wirbeltensor bezeichnet.

\subsubsection{Trennung von Gestalt- und Volumenänderung}

Für technische Elastomere ist die Annahme von nahezu inkompressiblem Materialverhalten, aufgrund des Unterschiedes um einige Größenordnungen zwischen Kompressions- und Schubmodul $K / G \gg 1$, gemeinhin anerkannt (vgl. Lion 2000, Wriggers 2001, Stommel \& Zimmermann 2011). Um dies zu modellieren, bietet es sich an, die Deformation als Volumen- und Gestaltänderung separat zu formulieren. Ein Zugang bietet hier die multiplikative Zerlegung des Deformationsgradienten (vgl. Kröner 1958, Sussman \& Bathe 1987). In der Gleichung (2-32) sind die zwei aufeinanderfolgenden Teildeformationen dargestellt.

$$
\underline{\underline{F}}=\underline{\underline{F}} \cdot \stackrel{V}{\underline{F}}
$$

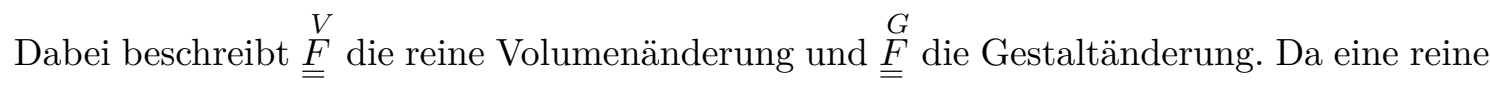
Volumenänderung nur dazu führt, dass alle materialfesten Linien entweder gleichmäßig gestaucht oder gestreckt werden, kann auch von einem hydrostatischen Tensor gesprochen werden (vgl. Ihlemann 2003). Der Gestaltänderungsanteil lässt sich dann über die Gesamtdeformation bestimmen.

$$
\stackrel{V}{\underline{F}}=\mu \underline{\underline{I}} \Rightarrow \stackrel{G}{\underline{F}}=\frac{1}{\mu} \underline{\underline{F}}
$$

Der Streckungsfaktor $\mu$ entspricht der dritten Hauptinvariante des Deformationsgradienten.

$$
\mu=\sqrt[3]{I_{3}(\underline{\underline{F}})}=J_{3}^{\frac{1}{3}}
$$

Nachfolgend können über die Zerlegung des Deformationsgradienten die Cauchy-GreenTensoren berechnet werden.

$$
\begin{gathered}
\underline{\underline{G}}=\underline{\underline{F}}^{T} \cdot \underline{\underline{F}}=\frac{1}{\mu^{2}} \underline{\underline{C}}=J_{3}^{-\frac{2}{3}} \underline{\underline{C}} ; \underline{\underline{C}}=\underline{\underline{F}}^{T} \cdot \underline{\underline{F}}=\mu^{2} \underline{\underline{I}}=J_{3}^{\frac{2}{3}} \underline{\underline{I}} \\
\stackrel{G}{\underline{\underline{b}}}=\underline{\underline{\underline{F}}} \cdot \underline{\underline{F}}^{T}=\frac{1}{\mu^{2}} \underline{\underline{b}}=J_{3}^{-\frac{2}{3}} \underline{\underline{b}} ; \quad \underline{\underline{b}}=\underline{\underline{F}} \cdot \underline{\underline{F}}^{T}=\mu^{2} \underline{\underline{I}}=J_{3}^{\frac{2}{3}} \underline{\underline{I}}
\end{gathered}
$$

Abschließend werden noch die ersten beiden Hauptinvarianten, wie sie beispielsweise zur Herleitung hyperelastischer Stoffgesetze mit quasi-inkompressibler Formulierung benötigt werden (siehe nächster Abschnitt 2.1.4), mit $J_{1}$ und $J_{2}$ angegeben.

$$
J_{1}=I_{1}(\underline{\underline{G}})=I_{1}(\underline{\underline{G}})=J_{3}^{-\frac{2}{3}} I_{1}(\underline{\underline{C}}) ; \quad J_{2}=I_{2}(\underline{\underline{G}})=I_{2}(\underline{\underline{b}})=J_{3}^{-\frac{4}{3}} I_{2}(\underline{\underline{C}})
$$




\subsubsection{Hyperelastische Stoffgesetze mit quasi-inkompressibler Formulierung}

Ausgangspunkt zur Herleitung von Stoffgesetzen bilden die Hauptsätze der Thermodynamik. Eine umfassende Darstellung der verschiedenen Bilanzgleichungen kann dabei einschlägiger Literatur entnommen werden (vgl. Holzapfel 2000, Haupt 2002). Eine besondere Stellung nimmt die Clausius-Duhem-Ungleichung ein, die auch als lokale Form des zweiten Hauptsatzes der Thermodynamik bezeichnet wird.

$$
[\underline{\underline{\underline{T}}} \cdot \stackrel{\underline{\underline{\gamma}}}{=}-\tilde{\rho}(\dot{\psi}+\dot{\theta} \eta)]-\frac{1}{\theta} \underline{\widetilde{q}} \cdot \underline{\widetilde{\nabla}} \theta \geq 0
$$

Die Größen $\dot{\psi}$ und $\dot{\theta}$ kennzeichnen hierbei die zeitlichen Ableitungen der freien HelmholtzEnergie und der absoluten Temperatur. Die Variable $\eta$ beschreibt die spezifische Entropie und die Größe $\widetilde{q}$ die Wärmestromdichte in der Referenzkonfiguration. Für den Sonderfall, dass nur mechanische Dissipation stattfindet, erhält man die Clausius-Planck-Ungleichung. Da im Folgenden hyperelastische Stoffgesetze mit Beschränkung auf isothermen und adiabaten Vorgängen hergeleitet werden (keine Energiedissipation), kann aus der ClausiusPlanck-Ungleichung eine Gleichgewichtsgleichung formuliert werden:

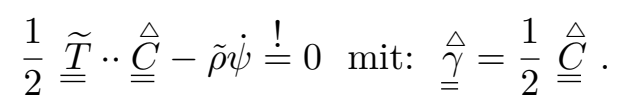

Neben der lagrangeschen Zeitableitung für den Rechten Cauchy-Green-Tensor wird auch die zeitliche Ableitung der freien Energiedichte benötigt. Für Stoffgesetze mit schwacher Kompressibilität wird die Energiedichte additiv aus einem Gestalt- und einem Volumenänderungsanteil zusammengesetzt:

$$
\tilde{\rho} \psi=\tilde{\rho} \psi_{G}\left(J_{1}, J_{2}\right)+\tilde{\rho} \psi_{V}\left(J_{3}\right)
$$

Die partielle Ableitung der freien Energiedichte nimmt dabei folgende Form an:

$$
\begin{aligned}
& \tilde{\rho} \dot{\psi}=\frac{\partial \tilde{\rho} \psi_{G}}{\partial J_{1}} \dot{J}_{1}+\frac{\partial \tilde{\rho} \psi_{G}}{\partial J_{2}} \dot{J}_{2}+\frac{\partial \tilde{\rho} \psi_{V}}{\partial J_{3}} \dot{J}_{3} \text { mit: }
\end{aligned}
$$

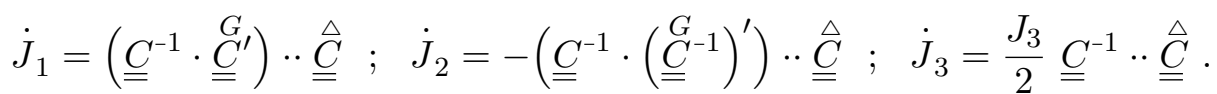

Setzt man diese Ableitung der freien Energiedichte und die Ableitungen der $J$-Invarianten $\dot{J}_{1}, \dot{J}_{2}, \dot{J}_{3}$ (s. Ihlemann 2003) in die Claudius-Planck-Gleichung ein, so entsteht folgender Ausdruck:

$$
\left[\frac{1}{2} \underline{\underline{\underline{T}}}-\left(\frac{\partial \tilde{\rho} \psi_{G}}{\partial J_{1}} \underline{\underline{C}}^{\prime}-\frac{\partial \tilde{\rho} \psi_{G}}{\partial J_{2}}\left(\underline{\underline{G}}^{-1}\right)^{\prime}\right) \cdot \underline{\underline{C}}^{-1}-\frac{J_{3}}{2} \frac{\partial \tilde{\rho} \psi_{V}}{\partial J_{3}} \underline{\underline{C}}^{-1}\right] . . \stackrel{\underline{\underline{C}}}{\underline{\underline{a}}}=0
$$




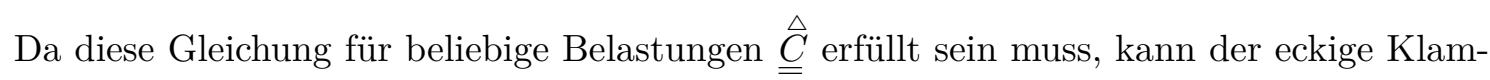
merausdruck zu Null gesetzt werden. Es entsteht folgende Gleichung:

$$
\forall \underline{\underline{C}}: \quad \underline{\underline{\widetilde{T}}}=2\left(\frac{\partial \tilde{\rho} \psi_{G}}{\partial J_{1}} \underline{\underline{G}}-\frac{\partial \tilde{\rho} \psi_{G}}{\partial J_{2}} \underline{\underline{G}}^{-1}\right)^{\prime} \cdot \underline{\underline{C}}^{-1}+J_{3} \frac{\partial \tilde{\rho} \psi_{V}}{\partial J_{3}} \underline{\underline{C}}^{-1}
$$

Mit Hilfe des Übertragungsoperators, der in Ihlemann (2003) eingeführt wurde, kann eine Größe aus der Referenzkonfiguration in die aktuelle Konfiguration übertragen werden: $S^{-1}((\underline{\underline{X}}))=\underline{\underline{F}} \cdot \underline{\underline{X}} \cdot \underline{\underline{F}}^{-1}$. Somit kann der 2. Piola-Kirchhoff-Spannungstensor in den CauchySpannungstensor überführt werden:

$$
\underline{\underline{\sigma}}=\frac{2}{J_{3}}\left(\frac{\partial \tilde{\rho} \psi_{G}}{\partial J_{1}} \underline{\underline{b}}^{G}-\frac{\partial \tilde{\rho} \psi_{G}}{\partial J_{2}} \underline{\underline{b}}^{-1}\right)^{\prime}+\frac{\partial \tilde{\rho} \psi_{V}}{\partial J_{3}} \underline{\underline{I}} .
$$

Im Weiteren sollen Ansätze für isotrope hyperelastische Stoffgesetze mit schwacher Kompressibilität vorgestellt und diskutiert werden. Zunächst wird dazu ein EnergiedichteAnsatz gewählt, aus dem sich verschiedenen Grundformen ableiten lassen (vgl. Rivlin \& Saunders 1951):

$$
\tilde{\rho} \psi_{G}=\sum_{i=0}^{m} \sum_{j=0}^{n} c_{i j}\left(J_{1}-3\right)^{i}\left(J_{2}-3\right)^{j}
$$

Mit dem alleinigen Parameter: $c_{10}$ erhält man den Ansatz für Neo-Hooke (1943), für $c_{10}, c_{01}$ den für Mooney-Rivlin (1940) und für $c_{10}, c_{20}, c_{30}$ den für Yeoh (1993). Zur Berücksichtigung der Volumenänderung gibt es eine Vielzahl verschiedener EnergiedichteAnsätze, welche in Hartmann (2003) aufgelistet sind. Die dabei in der Literatur am Häufigsten zur Anwendung kommende Vertreter sind mit $\tilde{\rho} \psi_{V 1}$ und $\tilde{\rho} \psi_{V 2}$ angegeben. Für das im Rahmen dieser Arbeit verwendete FE-Programm Msc.Marc kommt standardmäßig der Ansatz $\tilde{\rho} \psi_{V 3}$ zum Einsatz:

$$
\tilde{\rho} \psi_{V 1}=\frac{K}{2}\left(J_{3}-1\right)^{2} ; \quad \tilde{\rho} \psi_{V 2}=\frac{K}{2}\left(\ln J_{3}\right)^{2} ; \quad \tilde{\rho} \psi_{V 3}=\frac{9 K}{2}\left(J_{3}^{1 / 3}-1\right)^{2} .
$$

Exemplarisch wird der Energiedichte-Ansatz von Yeoh mit schwacher Kompressibilität (Ansatz: $\tilde{\rho} \psi_{V 3}$ ) weiter verwendet. Die Gesamtenergiedichte nimmt dabei folgende Form an (mit Berücksichtigung des Volumenanteils):

$$
\tilde{\rho} \psi=c_{10}\left(J_{1}-3\right)+c_{20}\left(J_{1}-3\right)^{2}+c_{30}\left(J_{1}-3\right)^{3}+\frac{9 K}{2}\left(J_{3}^{1 / 3}-1\right)^{2} .
$$

Die benötigten Ableitungen ergeben sich dabei zu:

$$
\frac{\partial \tilde{\rho} \psi_{G}}{\partial J_{1}}=c_{10}+2 c_{20}\left(J_{1}-3\right)+3 c_{30}\left(J_{1}-3\right)^{2} ; \quad \frac{\partial \tilde{\rho} \psi_{G}}{\partial J_{2}}=0 ; \quad \frac{\partial \tilde{\rho} \psi_{V}}{\partial J_{3}}=3 K\left(J_{3}^{1 / 3}-1\right) J_{3}^{-2 / 3} .
$$


Setzt man diese Ableitungen in die Gleichung (2-45) ein, so erhält man das Yeoh-Stoffgesetz mit quasi-inkompressibler Formulierung:

$$
\underline{\underline{\sigma}}=\frac{2}{J_{3}}\left(c_{10}+2 c_{20}\left(J_{1}-3\right)+3 c_{30}\left(J_{1}-3\right)^{2}\right) \underline{\underline{\underline{b}}}^{G}+3 K\left(J_{3}^{1 / 3}-1\right) J_{3}^{-2 / 3} \underline{\underline{I}} .
$$

In späteren Abschnitten werden diese analytischen Gleichungen für die Berechnung von Fehlermaßen benötigt. Beispielsweise kann ein Spannungsmaß definiert werden, welches die Abweichung zwischen aktuell vorliegender und theoretisch exakter Lösung beschreibt. Dadurch können in direkter Weise Aussagen zur Güte eines Messbereiches getroffen werden.

\subsection{Stand der Technik zu Elastomer-Probekörpern}

Die Bestimmung der physikalischen Eigenschaften von Elastomeren lässt sich bis in die frühen 60er Jahre zu J.R. Scott zurückführen. Seitdem existieren eine Vielzahl von Prüfmethoden, internationalen Standards und Probekörpern, die es ermöglichen die komplexen Eigenschaften von Elastomeren zu erfassen. In Brown (2006) befindet sich eine ausführliche Beschreibung zur Präparation von Probekörpern, deren Versuchsdurchführung und Auswertung. Dabei werden grundlegende Zusammenhänge zur Charakterisierung von technischen Elastomeren erläutert, Messstrategien beschrieben und die verschiedenen Herangehensweisen diskutiert. Die darin beschriebenen nationalen und internationalen Standards legen wichtige Grundsteine für die Verbesserung von Produkten im Industriealltag, die Sicherstellung der Qualität im Herstellungsprozess und die Sammlung von Kennwerten für Materialdatenbanken (vgl. Brown 2006).

\subsubsection{Probekörper für einachsige Zug-/Druckversuche}

Im Folgenden werden eine Reihe von Probekörpern vorgestellt, wie sie zur Charakterisierung von technischen Elastomeren Anwendung finden. Dazu soll zunächst ein Überblick über die Hauptvertreter der verschiedenen Belastungsarten gegeben werden. Einer der Grundversuche stellt dabei der einachsige Zug- bzw. Druckversuch dar. In der Abbildung 2.1 sind zum besseren Verständnis verschiedene Zug- oder/und Druckprobekörper dargestellt. Der S2-Stab ist einer der bekanntesten Vertreter zur Untersuchung des Materialverhaltens im Zugbereich. Seine Geometrie ist dahingehend optimiert, dass ein möglichst großer homogener Messbereich vorhanden ist (vgl. Brown 2006, Kottner u.a. 2018). Idealerweise erfolgt die Dehnungsmessung in dem verjüngten Bereich mittels eines Längenänderungsaufnehmers. Hierfür eignet sich als berührendes System beispielsweise der MultiXtens der Firma Zwick/Roell. Analog dazu bieten sich auch optische Methoden, wie die Dehnungsmessung mittels Laserextensometer oder Grauwertkorrelation an. Die verschiedenen Messsysteme weisen dabei ganz unterschiedliche Vor- und Nachteile auf und sollten daher je nach Anforderungsprofil der Messaufgabe ausgewählt werden. 


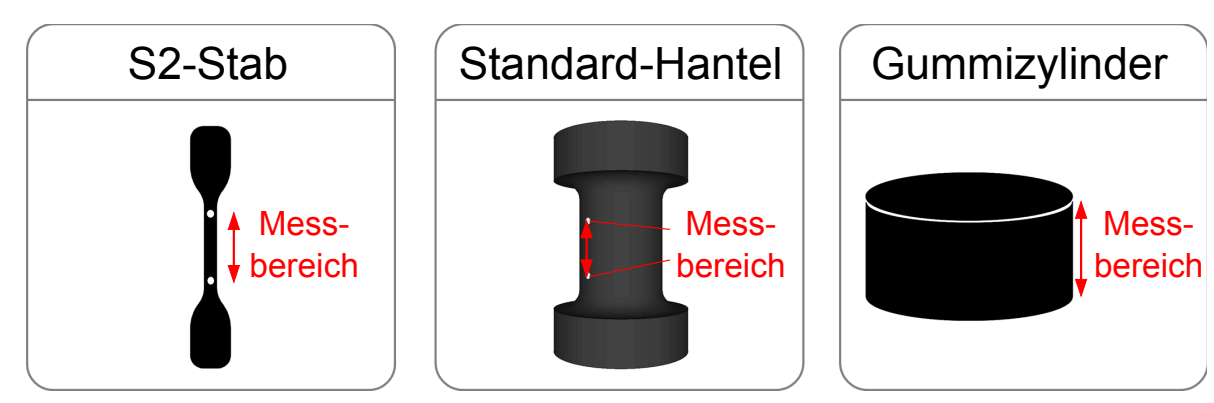

\begin{abstract}
Abb. 2.1 - Gegenüberstellung verschiedener Probekörper für einachsige Zug/Druckversuche. Die Abbildung ist in leicht modifizierter Form Kanzenbach u. a. (2018) entnommen. Wiederverwendung mit Genehmigung. () 2018 Elsevier Ltd.
\end{abstract}

Neben Vorteilen wie der einfachen Geometrie und dem großen homogenen Messbereich besitzt der S2-Stab einen entscheidenden Nachteil. Der Probekörper ist in keiner Weise für Druckbeanspruchung geeignet. Dabei stellt aber gerade diese eine wichtige Beanspruchungsart dar. In der Arbeit von Bergström (1999) wird die vereinfachte Geometrie eines Druckprobekörpers vorgestellt. Dieser Gummizylinder (siehe Abbildung 2.1, rechts) besitzt aufgrund seiner kompakten Struktur den entscheidenden Vorteil, dass er auch für größere Druckbelastungen geeignet ist. Allerdings kann dieser Probekörper nicht für Zugversuche herangezogen werden. Hinzu kommt, dass die Stirnflächen des Probekörpers sehr gut geschmiert sein müssen, da sich sonst aufgrund der hohen Reibung ein sehr inhomogener Deformationszustand einstellt (vgl. Stańco \& Działak 2018). Um sowohl Zug- als auch Druckversuche realisieren zu können, empfiehlt sich die Verwendung einer hantelförmigen Probekörpergeometrie. In der Arbeit von Lion (2000), als auch in der Veröffentlichung von Alshuth u.a. (2007) werden dazu zwei verschiedene Ausführungen der Einspannung vorgestellt (vgl. Abbildung 1.1). Bei Lion (2000) erfolgt die Einspannung der Probenenden durch Kleben in Metalltöpfe, die dann spielfrei in die dafür vorgesehenen Halterungen der Prüfmaschine eingebaut werden. Im Vergleich dazu wird bei Alshuth u. a. (2007) der Kopf der Standard-Hantel über Halterungsadapter mit der Prüfmaschine verbunden. Der große Vorteil beider Ausführungen besteht darin, dass jetzt erstmals kombinierte ZugDruckversuche realisiert werden können. Allerdings sei hier explizit darauf hingewiesen, dass der Probekörper bei Stauchungen größer 30 \% zu sehr inhomogenen Deformationen führt (vgl. Abbildung 1.1, rechts).

\title{
2.2.2 Probekörper für biaxiale Versuche
}

Eine weitere interessante Beanspruchungsart stellt der äquibiaxiale Zug dar. Diese Belastungsart führt im Idealfall zu identischen Verzerrungszuständen wie der einachsige Druckversuch. In der Veröffentlichung von Alshuth u.a. (2007) erfolgte der Vergleich zwischen einem umgerechneten äquibiaxialen Zugversuch und einem Druckversuch mittels Hantelprobekörper. Hierbei stellte sich heraus, dass beim Äquibiaxialversuch schmalere Hysteresen zu verzeichnen sind und damit eine geringere Energiedissipation stattfindet. Diese 
Abweichungen können aus dem Äquibiaxialversuch selbst resultieren oder aus dem Druckversuch mit dem Hantelprobekörper, der bei größeren Stauchungen inhomogene Deformationen aufweist. In den Veröffentlichungen von Johlitz \& Diebels (2011) und Seibert u. a. (2014) wird ein verbesserter biaxialer Zugprobekörper vorgestellt (vgl. Abbildung 2.2. Die Probekörpergeometrie (Kreuzform mit parametrisiertem Radius) wurde dabei mittels

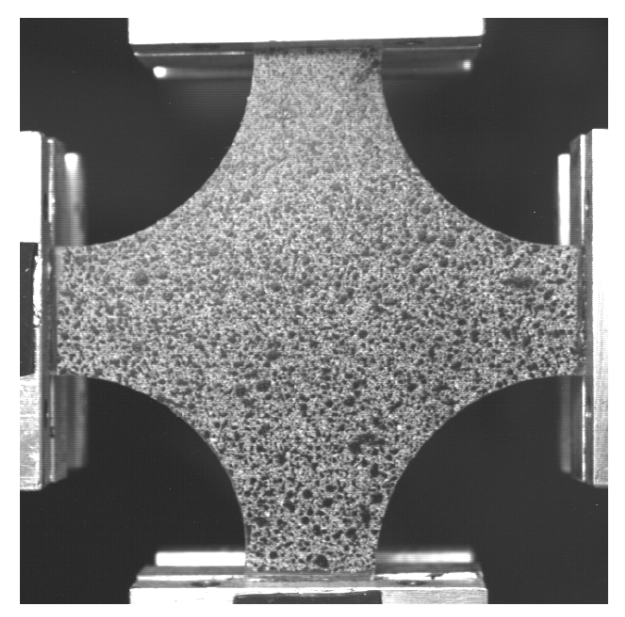

Abb. 2.2 - Biaxialer Zugprobekörper (Abbildung aus der Veröffentlichung von Seibert u. a. 2014)

verschiedener Qualitätskriterien experimentell optimiert.

\subsubsection{Scherprobekörper}

Eine ganz andere Grundbeanspruchungsart stellt die einfache Scherung dar. Als einer der bekanntesten Vertreter ist der zylindrische Double-Shear-Probekörper, wie er im Forschungsinstitut TARRC (Hertford) eingesetzt wird, zu nennen. In den Veröffentlichungen von Besdo u. a. (2003 \& 2005) und Ahmadi u. a. (2007) ist dieser symmetrische Scherprobekörper abgebildet. Das Design beruht auf einer Zylindergeometrie, die an den Stirnflächen anvulkanisiert oder angeklebt wird. Der Aufbau erfolgt in doppelt symmetrischer Form, um auftretende Querkräfte zu kompensieren (vgl. Abbildung 2.3). In den oben genannten Veröffentlichungen finden sich weiterhin eine Vielzahl von Versuchen zur Untersuchung des Relaxations- und Erholungsverhaltens technischer Elastomere (vgl. auch Lampe u. a. 2018). Eine Weiterentwicklung dieses Scherprobekörpers für Lebensdaueruntersuchungen findet sich in Freund (2007). Die Probekörpergeometrie wurde hierbei durch eine geeignete Kombination der beiden Rundungsradien der Kontakt- und Manteloberfläche angepasst, um einen möglichst homogenen Bereich mit maximalen Spannungen im Innern des Körpers zu erzielen. In der Arbeit von Klauke (2015) wird dieser optimierte Scherprobekörper dann zur Lebensdauervorhersage unter Berücksichtigung rotierender Beanspruchungsrichtungen angewendet.

Ein weiterer Vertreter ist der Scherprobekörper von der Firma Akzo Nobel. Das De- 
sign beruht hier auf einem flächigen Scherprobekörper, der zwischen drei Metallleisten eingeklemmt wird. Die mittlere Metallleiste dient zur Lasteinleitung und wird demzufolge von der Traverse verfahren. In der Abbildung 2.3 sind die vorgestellten Scherprobekörper dargestellt. Im Weiteren soll auf die verschiedenen Vor- und Nachteile der be-
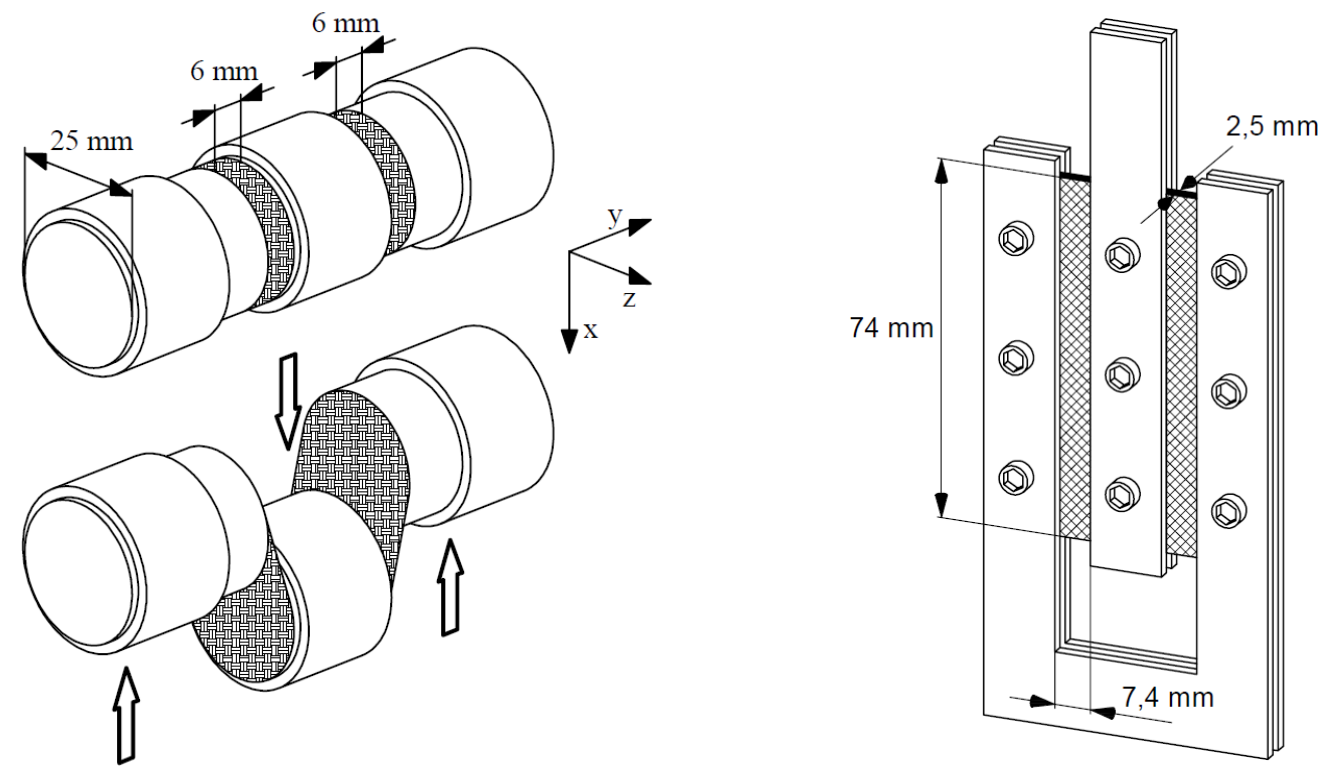

Abb. 2.3 - Gegenüberstellung zweier Scherprobekörper (links) zylindrischer Double-Shear-Probekörper (Abbildung aus der Veröffentlichung von Besdo u. a. 2003) und (rechts) flächiger Scherprobekörper von der Firma Akzo Nobel (Abbildung aus der Arbeit von Ihlemann 2003)

schriebenen Scherprobekörper näher eingegangen werden. Dabei soll zunächst besonderes Augenmerk auf die Grundgeometrie der Scherprobekörper gelegt werden. Der DoubleShear-Probekörper bietet den großen Vorteil, dass er aufgrund seiner kompakten Struktur (geringes Höhen-Durchmesserverhältnis) sehr gut an den Stirnflächen anvulkainsiert oder geklebt werden kann. Dadurch wird die Scherdeformation in einem hohem Maße sehr gut übertragen. In der Arbeit von Freund (2007) konnte gezeigt werden, dass der Probekörper über weite Bereiche homogen deformiert wird. Lediglich in den Randbereichen sind größer werdende Inhomogenitäten der Von-Mises-Vergleichsspannung zu verzeichnen. Als wesentlicher Nachteil ist das Auftreten von hohen Spannungen im Kontaktbereich zu nennen. Aus diesem Grund schlägt Freund (2007) eine Taillierung der Stirn- und Zylinderflächen vor. Nachteilig bleibt aber, dass durch die Klebe- oder Vulkanisationsverbindung zwischen dem Probekörper und den metallischen Anschlussteilen eine Beeinflussung der Materialeigenschaften im Kontaktbereich stattfindet. Ein weiterer Nachteil ist das Entstehen von Schrumpf und das Auftreten von Eigenspannungen nach dem Anvulkanisieren. Diese Einflüsse können nur bedingt minimiert werden.

Als nächstes werden die prinzipiellen Vor- und Nachteile von flächigen Scherprobekörpern beschrieben. Hierbei wird zunächst auf die verschiedenen Anwendungsmöglichkeiten 
näher eingegangen. Flächige Scherprobekörper besitzen den großen Vorteil, dass mit vergleichsweise geringem Aufwand Scherversuche mit Normalkraft Null durchgeführt werden können. Weiterhin ermöglichen flächige Scherprobekörper aufgrund der großen freien Oberflächen homogene Alterungsuntersuchungen. Solche Untersuchungen sollten beispielsweise mit dem Double-Shear-Probekörper vermieden werden, da hier große Bereiche anvulkanisiert oder geklebt sind und dadurch kein Sauerstoff mit der Oberfläche reagieren kann. Demzufolge stellt sich hier eine sehr inhomogene Alterung ein, die auf bestimmte Bereiche nahe der freien Oberfläche beschränkt ist. Flächige Scherprobekörper besitzen darüber hinaus den Vorteil, dass der Einfluss der Anisotropie nach einer Vorreckung auf Zug untersucht werden kann. Ebenso ist es denkbar, aus den gescherten Gummimatten S2-Stäbe für einen anschließenden Zugversuch zu entnehmen, bzw. parallel zu den Scherversuchen gleich Zugversuche aus dem gleichen Material durchzuführen. Durch die Verwendung konventioneller Gummimatten bietet sich auch die Möglichkeit an, faserverstärkte Materialien zu testen. Ein prinzipieller Nachteil von flächigen Scherprobekörpern ist, dass bei größeren Deformationen ein erhöhtes Risiko von Beulerscheinungen besteht. Diesem Umstand kann aber durch ein optimiertes Breiten-Dickenverhältnis begegnet werden. Allerdings sollte bei der Dimensionierung der Dicke darauf geachtet werden, dass noch eine homogene Alterung stattfinden kann. Die Verwendung von herkömmlichen Gummimatten hat auch den ganz praktischen Vorteil, dass die Herstellung von Probekörpern verhältnismäßig einfach und kostengünstig ist. Die Gummimatten können beispielsweise dem Serienmaterial entnommen oder als Platten gepresst werden. 



\section{Probekörperdesign für hochpräzise Zug-/Druckmessungen}

Das Kapitel beschäftigt sich mit der Entwicklung eines hochpräzisen Zug-/Druckprobekörpers für die Charakterisierung von Elastomerwerkstoffen. Von der Idee des speziellen Designs der Halterungen, bis hin zur Geometrieoptimierung des Probekörpers und der experimentellen Erprobung, ist der komplette Entwicklungszyklus aufgeführt. Mit der industriellen Anwendung des Probekörper-Setups findet die Entwicklung einen erfolgreichen Abschluss. Das spezielle Design der Halterungen ermöglicht es, auch bei extremen Stauchungen ein nahezu homogenes Materialverhalten zu messen. Der neu entwickelte Probekörper eignet sich somit ideal für die phänomenologische Untersuchung von Elastomerwerkstoffen.

\subsection{Motivation für die Entwicklung kombinierter Zug-/Druckprobekörper}

Die Anforderungen an neue Probekörper hinsichtlich Funktionalität, Präzision, Homogenität und Wirtschaftlichkeit, wachsen in gleichem Maße wie die Notwendigkeit neuer Stoffgesetze. Dabei finden Probekörper in ganz unterschiedlichen Bereichen Anwendung:

- Überprüfung des Produktionsprozesses

- Identifikation von Materialparametern

- Untersuchung grundlegender phänomenologischer Eigenschaften

- Funktionstauglichkeit bei der Produktentwicklung

Elastomerwerkstoffe sind aus vielen Bereichen der Automobilindustrie nicht mehr wegzudenken, beispielhaft dafür sind Fahrwerksbuchsen, Reifen oder Dämpfer. Von besonderem Interesse sind Probekörper, die für kombinierte Zug- und Druckbelastungen geeignet sind. Solche Probekörper können dann zur Untersuchung des Materialverhaltens von Elastomeren als auch bei der Identifikation von Materialparametern genutzt werden. Die Erhöhung der Homogenität der Spannungs- und Verzerrungsverteilung im Messbereich stellt eine genauso wichtige Optimierungsgröße wie die der Stauchung dar. Für einachsige homogene Zugbeanspruchungen kann beispielsweise ein S2-Stab verwendet werden. 
Der große Nachteil besteht darin, dass der Probekörper in keiner Weise für Druckbeanspruchungen geeignet ist. In vielen technischen Anwendungen spielen aber gerade Druckbeanspruchungen eine wichtige Rolle, beispielhaft dafür sind Fahrwerksbuchsen oder Erdbebendämpfer. Um eine geeignete Materialprüfung und Identifizierung von Materialparametern durchführen zu können, benötigt man also einen Probekörper, der auch für Druckbeanspruchungen geeignet ist. Hier liegt die Idee nahe, einen Hantelprobekörper zu verwenden. Allerdings treten dabei zwei gegensätzliche Phänomene auf. Einerseits soll der Probekörper möglichst lang gestaltet sein, um eine möglichst homogene Deformation im Mittelbereich zu erzielen dies führt aber schon bei kleinsten Imperfektionen zum Knicken. Andererseits würde ein Probekörper mit sehr großem Durchmesser und geringer/kleiner Höhe zwar eine hohe Knicksicherheit aufweisen, dafür aber zu einer inhomogenen Deformation im Messbereich führen. Ziel ist es also, einen Probekörper zu gestalten, der sowohl für große Stauchungen als auch für eine homogene Deformation im Messbereich geeignet ist. Erreicht werden soll diese herausragende Eigenschaftskombination durch ein spezielles Design der Probekörperhalterungen. In der Veröffentlichung von Alshuth u. a. (2007) beschreiben die Autoren den Hantelprobekörper, wie er zurzeit im Deutschen Institut für Kautschuktechnologie e.V. verwendet wird.

\subsection{Analytische Untersuchungen zur Knickstabilität von Stäben und Balken}

\subsubsection{Differentialgleichung der Balkenschwingung}

Im Folgenden werden eine Reihe analytischer Untersuchungen zur Knickstabilität von Stäben und Balken durchgeführt. Dabei soll insbesondere analysiert werden, was charakteristische Größen zur Beschreibung der Knicksicherheit bzw. Knickstabilität sind. Diese analytischen Untersuchungen dienen als Vorbereitung und Überprüfung numerischer Verfahren zur Entwicklung von Halterungsgeometrien, wie sie in Kapitel 3.3 vorgestellt werden. Dafür wird zunächst die Differentialgleichung für den allgemeinen Fall einer Balkenschwingung hergeleitet. Mit Hilfe dieser Differentialgleichung sollen eine Reihe von Sonderfällen, wie sie für diese Arbeit interessant sind, näher untersucht werden. In der Abbildung 3.1 ist das Freikörperbild für ein infinitesimales Balkenelement dargestellt. Stellt man nun die Gleichgewichtsbedingung in vertikaler Richtung und das Momentengleichgewicht um den Schwerpunkt auf, so erhält man folgende Gleichungen (vgl. Dresig u.a. 2007, Gross u.a. 2007):

$$
\begin{aligned}
\mathrm{d} m \ddot{w} & =\left(Q_{z}+\mathrm{d} Q_{z}\right) \cos (\alpha+\mathrm{d} \alpha)-Q_{z} \cos \alpha+(N+\mathrm{d} N) \sin (\alpha+\mathrm{d} \alpha)-N \sin \alpha+q_{z} \mathrm{~d} s \\
\mathrm{~d} J \ddot{\alpha} & =\left(M_{y}+\mathrm{d} M_{y}\right)-M_{y}-\frac{\mathrm{d} s}{2}\left(Q_{z}+\mathrm{d} Q_{z}\right)-\frac{\mathrm{d} s}{2} Q_{z} .
\end{aligned}
$$




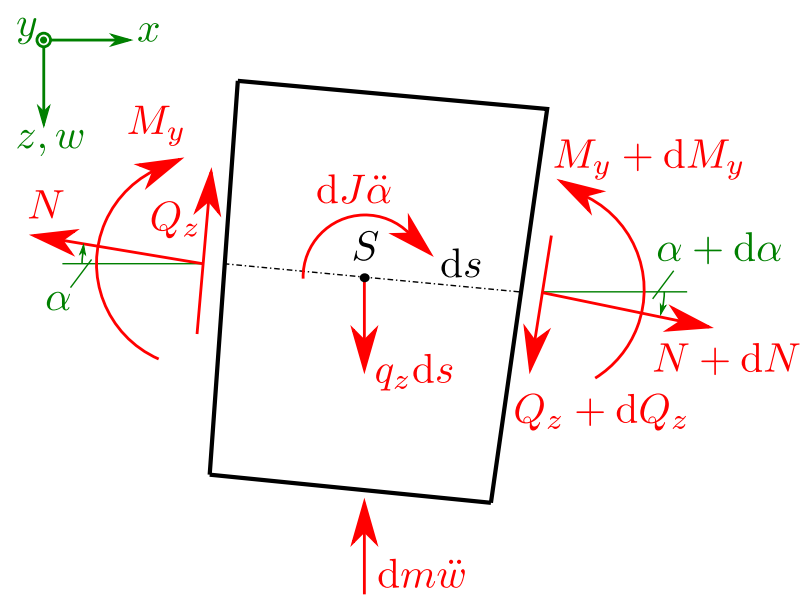

Abb. 3.1 - Freikörperbild eines infinitesimalen Balkenelements

Unter der Annahme kleiner Winkel und der Vernachlässigung von Größen zweiter Ordnung erhält man mit Hilfe von Additionstheoremen für den Schwerpunktsatz und den Drallsatz folgende Zusammenhänge:

$$
\begin{aligned}
\rho A \ddot{w} & =\left(N w^{\prime}\right)^{\prime}+Q_{z}^{\prime}+q_{z} \\
\rho I_{y} \ddot{w}^{\prime} & =M_{y}^{\prime}-Q_{z} .
\end{aligned}
$$

Die Querkraft $Q_{z}$ kann durch Ortsableitung des Drallsatzes und dessen Einsetzung in die Schwerpunktgleichung eliminiert werden. Es entsteht eine partielle Differentialgleichung der Ordnung 2:

$$
\rho A \ddot{w}=\left(N w^{\prime}\right)^{\prime}+M_{y}^{\prime \prime}-\left(\rho I_{y} \ddot{w}^{\prime}\right)^{\prime}+q_{z}
$$

Einfache Materialbeziehungen wie das Hooke'sche Stoffgesetz oder das Zener-Modell lassen sich über Äquivalenzbedingungen in die partielle Differentialgleichung integrieren (vgl. Gross u. a. 2007, Lei u.a. 2013):

$$
\begin{array}{ll}
M_{y}=-E I_{y} w^{\prime \prime} & \rightarrow \text { Hooke'sches Stoffgesetz } \\
M_{y}=-E_{\infty} I_{y} w^{\prime \prime}-\int_{-\infty}^{t} E_{1} I_{y} e^{-(t-s) / \tau_{1}} \frac{\partial w^{\prime \prime}}{\partial s} \mathrm{~d} s . & \rightarrow \text { Zener-Modell }
\end{array}
$$

Setzt man nun die Äquivalenzbedingung (3-6) in die Gleichung (3-5) ein, so erhält man die allgemeine Differentialgleichung der Balkenschwingung. Mit Hilfe der Gleichung (3-8) sollen nun einige Sonderfälle diskutiert werden.

$$
\rho A \ddot{w}=-\left(E I_{y} w^{\prime \prime}\right)^{\prime \prime}-\left(\rho I_{y} \ddot{w}^{\prime}\right)^{\prime}+\left(N w^{\prime}\right)^{\prime}+q_{z}
$$




\section{Sonderfall 1: Differentialgleichung der Saitenschwingung}

Um die allgemeine Differentialgleichung der Saitenschwingung zu erhalten, muss der Grenzfall $E I_{y} \rightarrow 0$ betrachtet werden. Neben der Biegesteifigkeit des Balkens in der Schwingungsebene $\left(E I_{y}=0\right)$ muss weiterhin die Rotationsträgheit $\left(\rho I_{y}=0\right)$ aus Gleichung $3-8$ vernachlässigt werden. Wird die Normalkraft als konstant vorausgesetzt ( $N=F=$ konst.), so entsteht die gesuchte Differentialgleichung:

$$
\rho A \ddot{w}-F w^{\prime \prime}-q_{z}=0
$$

\section{Sonderfall 2: Differentialgleichung der freien Balkenschwingung}

Als nächstes soll die Differentialgleichung der freien Balkenschwingung hergeleitet und diskutiert werden. Dazu wird die Rotationsträgheit $\left(\rho I_{y}=0\right)$ und die Flächenlast $q_{z}$ vernachlässigt. Es entsteht bei konstanter Normalkraft $(N=-F=$ konst.) folgender Zusammenhang:

$$
E I_{y} w^{\prime \prime \prime \prime}+\rho A \ddot{w}+F w^{\prime \prime}=0 .
$$

Aus Gleichung (3-10) kann erschlossen werden, dass Zugkräfte zu einer Erhöhung der Eigenfrequenzen des Balkens führen und Druckkräfte diese senken (vgl. Dresig u. a. 2007).

\section{Sonderfall 3: Differentialgleichung für die Stabilitätsanalyse von Balken bzw.} Stäben

Als letzter Sonderfall, zur Untersuchung des Stabilitätsverhaltens von Balken bzw. Stäben, wird zusätzlich der Trägheitsterm ( $\rho A=0$, Masse je Längeneinheit) vernachlässigt:

$$
E I_{y} w^{\prime \prime \prime \prime}+F w^{\prime \prime}=0
$$

\subsubsection{Störkraftuntersuchungen am Euler-IV-Druckstab}

Im Weiteren soll auf analytischem Wege untersucht werden, ob sich mit Hilfe von Störkräften ein Kriterium für die Sicherheit gegenüber Ausknicken definieren lässt. Um grundlegende Vergleiche (zu dem später auf numerischem Wege entwickelten Zug-/Druckprobekörper) zu ermöglichen, werden die Randbedingungen des Euler-IV Druckstabes gewählt. Für eine weitere Gegenüberstellung werden zunächst folgende Grundfälle unterschieden:

- einfacher Biegebalken (Fall 1)

- einfache Euler-IV-Knickung ohne Störkraft (Fall 2)

- einfache Euler-IV-Knickung mit Störkraft (Fall 3)

\section{Einfacher Biegebalken (Fall 1)}

Für den einfachen Fall des Biegebalkens kann die homogene Differentialgleichung der Form 
$w^{\prime \prime \prime \prime}=0$ durch triviale Integration gelöst werden. Die charakteristische Gleichung lautet wie folgt:

$$
w^{\prime \prime \prime \prime}=0 \Rightarrow w(x)=C_{1}+C_{2} x+C_{3} x^{2}+C_{4} x^{3}
$$

Die unbekannten Größen $C_{1}$ bis $C_{4}$ können über entsprechende Randbedingungen, siehe Euler-IV-Druckstab, gelöst werden:

$$
\begin{aligned}
w(0) & =0=C_{1} \\
w^{\prime}(0) & =0=C_{2} \\
w^{\prime}\left(\frac{l}{2}\right) & =0=C_{3} l+\frac{3}{4} C_{4} l^{2} \\
Q\left(\frac{l}{2}\right) & =S=-E I 6 C_{4} .
\end{aligned}
$$

Für den einfachen Fall des Biegebalkens entsteht folgende Form der Biegelinie mit der horizontalen Störkraft $2 S$ in der Symmetrieebene:

$$
w_{1}(x)=\frac{S l^{3}}{E I}\left[\frac{1}{8}\left(\frac{x}{l}\right)^{2}-\frac{1}{6}\left(\frac{x}{l}\right)^{3}\right] .
$$

\section{Einfache Euler-IV-Knickung ohne Störkraft (Fall 2)}

Als nächstes soll der Fall der einfachen Euler-IV-Knickung ohne Störkraft betrachtet werden. Die Differentialgleichung wurde bereits als Sonderfall 3 hergeleitet, siehe Gleichung (3-11):

$$
w^{\prime \prime \prime \prime}+\lambda^{2} w^{\prime \prime}=0 \text { mit: } \lambda^{2}=\frac{F}{E I} .
$$

Solche gewöhnlichen Differentialgleichungen können über den Ansatz:

$$
\begin{aligned}
w(x) & =C_{1} \cos (\lambda x)+C_{2} \sin (\lambda x)+C_{3} \lambda x+C_{4} \\
w^{\prime}(x) & =-C_{1} \lambda \sin (\lambda x)+C_{2} \lambda \cos (\lambda x)+C_{3} \lambda \\
w^{\prime \prime}(x) & =-C_{1} \lambda^{2} \cos (\lambda x)-C_{2} \lambda^{2} \sin (\lambda x) \\
w^{\prime \prime \prime}(x) & =C_{1} \lambda^{3} \sin (\lambda x)-C_{2} \lambda^{3} \cos (\lambda x) \\
w^{\prime \prime \prime \prime}(x) & =C_{1} \lambda^{4} \cos (\lambda x)+C_{2} \lambda^{4} \sin (\lambda x)
\end{aligned}
$$

gelöst werden (vgl. Gross u. a. 2007). Setzt man nun die Randbedingungen wie oben ein, 
so ergibt sich folgender Zusammenhang:

$$
\begin{aligned}
w(0) & =0=C_{1}+C_{4} \\
w^{\prime}(0) & =0=C_{2} \lambda+C_{3} \lambda \\
w^{\prime}\left(\frac{l}{2}\right) & =0=-C_{1} \lambda \sin \left(\lambda \frac{l}{2}\right)+C_{2} \lambda \cos \left(\lambda \frac{l}{2}\right)+C_{3} \lambda \\
Q\left(\frac{l}{2}\right) & =0=-E I w^{\prime \prime \prime}\left(\frac{l}{2}\right)-F w^{\prime}\left(\frac{l}{2}\right)=-F C_{3} \lambda \Rightarrow C_{3}=0 \Rightarrow C_{2}=0 .
\end{aligned}
$$

Aus der dritten Randbedingung kann für die nicht trivialen Fälle:

$$
-C_{1} \lambda \sin \left(\lambda \frac{l}{2}\right)=0 \Rightarrow \lambda \frac{l}{2}=n \pi
$$

gesetzt werden. Für die erste Knickform $(n=1)$ entsteht folgende Charakteristik der Biegelinie (einfache Euler-IV-Knickung ohne Störkraft):

$$
w_{2}(x)=C_{1}\left[\cos \left(2 \pi \frac{x}{l}\right)-1\right] .
$$

Ist man an der maximalen Verschiebung in Querrichtung interessiert, so erhält man die unbestimmte Größe der Auslenkung $C_{1}$ :

$$
w_{2, \max }=w_{2}\left(\frac{l}{2}\right)=-2 C_{1}=\bar{C}_{1} .
$$

Die kritische Kraft kann für den ersten auftretenden nichttrivialen Fall $\lambda_{\text {krit }} l / 2=\pi$ mit der Beziehung $\lambda^{2}=F / E I$ wie folgt berechnet werden:

$$
F_{2, \mathrm{krit}}=\frac{4 \pi^{2}}{l^{2}} E I
$$

\section{Einfache Euler-IV-Knickung mit Störkraft (Fall 3)}

Als letztes soll der Fall der einfachen Euler-IV-Knickung mit Störkraft betrachtet werden. Es sei für die Herleitung dieser Gleichungen darauf hingewiesen, dass die Annahme kleiner Verformungen berücksichtigt wurde. Für große Verformungen, wie sie nach dem Knicken auftreten, sei auf die geometrisch exakte Beschreibung Eulerscher Elastika verwiesen. In der Arbeit von Virgin (2007) finden sich beispielsweise ausführliche Untersuchungen zum Nachbeulverhalten (engl. post buckling). Für die hier durchgeführten Untersuchungen wird im Weiteren die Differentialgleichung, siehe Gleichung (3-14), verwendet. Die ersten drei Randbedingungen haben dabei die gleiche Form wie bei der Euler-IV-Knickung ohne Störkraft. Lediglich die Querkraft-Randbedingung hat die Störkraftgröße $S$, woraus sich von 
Null verschiedene Größen für die Unbekannten $C_{3}$ und $C_{2}$ ergeben:

$$
Q\left(\frac{l}{2}\right)=S=-F C_{3} \lambda \quad \Rightarrow \quad C_{3}=-\frac{S}{E I \lambda^{3}} \quad \Rightarrow \quad C_{2}=\frac{S}{E I \lambda^{3}} .
$$

Aus der dritten Randbedingung kann nun der nicht länger unbestimmte Faktor $C_{1}$ berechnet werden:

$$
C_{1}=\frac{S}{E I \lambda^{3}} \frac{\cos \left(\lambda \frac{l}{2}\right)-1}{\sin \left(\lambda \frac{l}{2}\right)} .
$$

Die Biegelinie der gestörten Knickform nimmt dabei folgende Charakteristik an:

$$
w_{3}(x)=\frac{S}{F}\left[\frac{\cos \left(\lambda \frac{l}{2}\right)-1}{\lambda \sin \left(\lambda \frac{l}{2}\right)}(\cos (\lambda x)-1)+\frac{1}{\lambda} \sin (\lambda x)-x\right] .
$$

Die maximale Auslenkung tritt an der Stelle $x=l / 2$ auf:

$$
w_{3, \max }=w_{3}\left(\frac{l}{2}\right)=\frac{S}{F}\left[2\left(1-\cos \left(\lambda \frac{l}{2}\right)\right)-\lambda \frac{l}{2} \sin \left(\lambda \frac{l}{2}\right)\right] \frac{1}{\lambda \sin \left(\lambda \frac{l}{2}\right)} .
$$

Aus der Gleichung (3-18) wird ersichtlich, dass die Auslenkung in der Mitte des Stabes beliebig anwächst, wenn der Nenner verschwindet $\lambda_{\text {krit }} l / 2=\pi$.

$$
F_{3, \mathrm{krit}}=\frac{4 \pi^{2}}{l^{2}} E I
$$

Die kritische Kraft hat offensichtlich das gleiche Maß wie bei der Euler-IV-Knickung ohne Störkraft. Unterschiede ergeben sich bei Betrachtung der auftretenden Verschiebungen in Querrichtung. Hier kann festgestellt werden, dass für den Euler-IV-Druckstab ohne Störkraft, unterhalb der kritischen Kraft keinerlei Verschiebung in Querrichtung auftritt und für den Euler-IV-Druckstab mit Störkraft dagegen große Verschiebungen. Aus den dargestellten Knickfällen lassen sich eine Reihe interessanter Schlussfolgerungen ziehen, die im Folgenden näher diskutiert werden sollen.

\section{Schlussfolgerungen der Stabilitätsanalysen:}

Zunächst kann festgestellt werden, dass die Größe der kritischen Kraft gänzlich unabhängig von der Größe der Störung $S$ ist. In Abbildung 3.2 ist dieser Sachverhalt für drei verschiedene Störgrößen grafisch dargestellt. Aus der Abbildung lässt sich entnehmen, dass die Störkraft einen Einfluss auf die Auslenkung in der Stabmitte hat, jedoch nicht auf die kritische Kraft selbst. Alle drei Kurven schmiegen sich asymptotisch an den horizontalen Verlauf der kritischen Kraft an. Als nächstes sollen die verschiedenen Verläufe der Biegelinien miteinander verglichen und diskutiert werden. Um eine einheitliche Darstellung der gestörten und ungestörten Knickverläufe zu ermöglichen, werden die Biegelinien durch ihren betragsmäßig größten Wert geteilt. Die normierten Verläufe nehmen dabei 


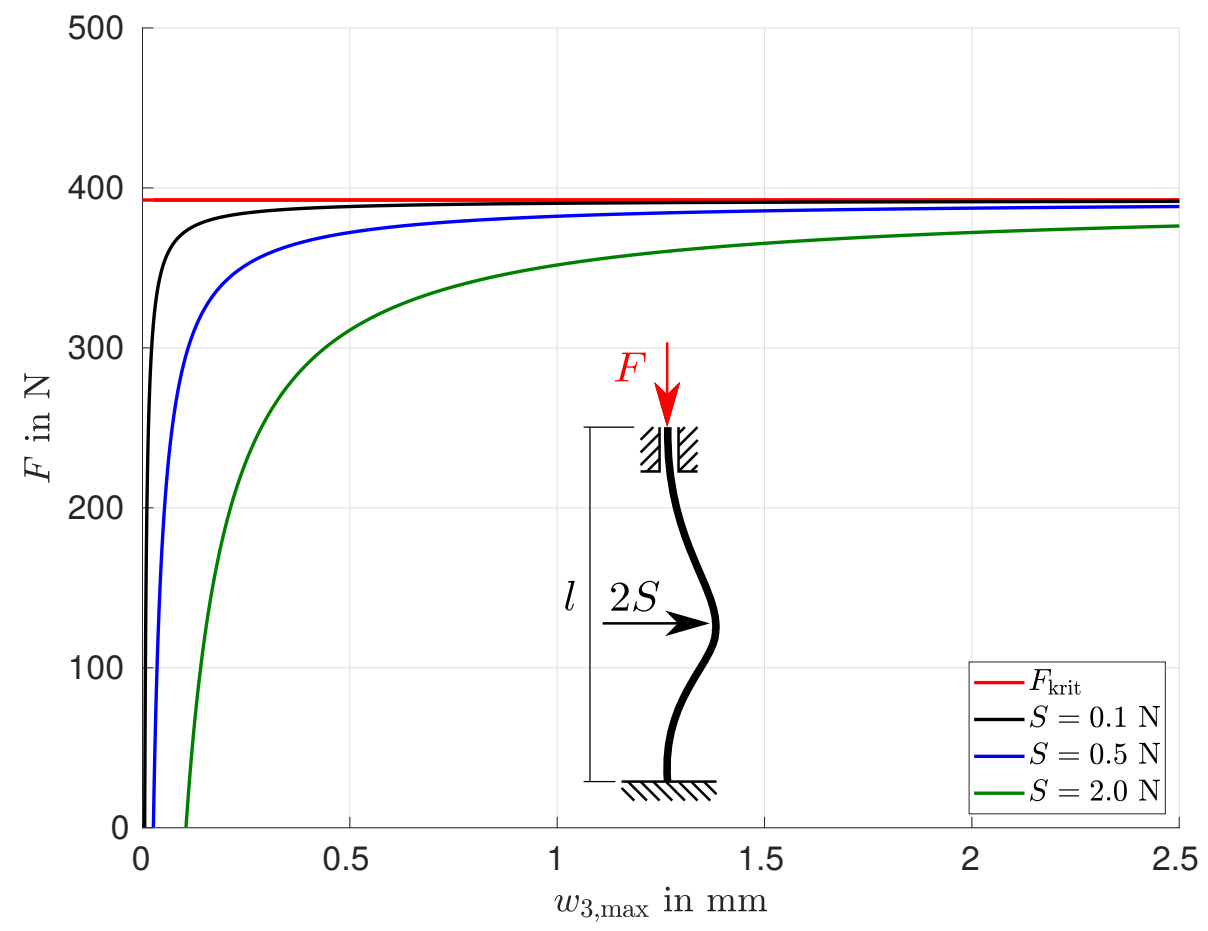

Abb. 3.2 - Störgröße $S$ als Maß zum Erreichen der kritischen Kraft (Simulationsergebnisse)

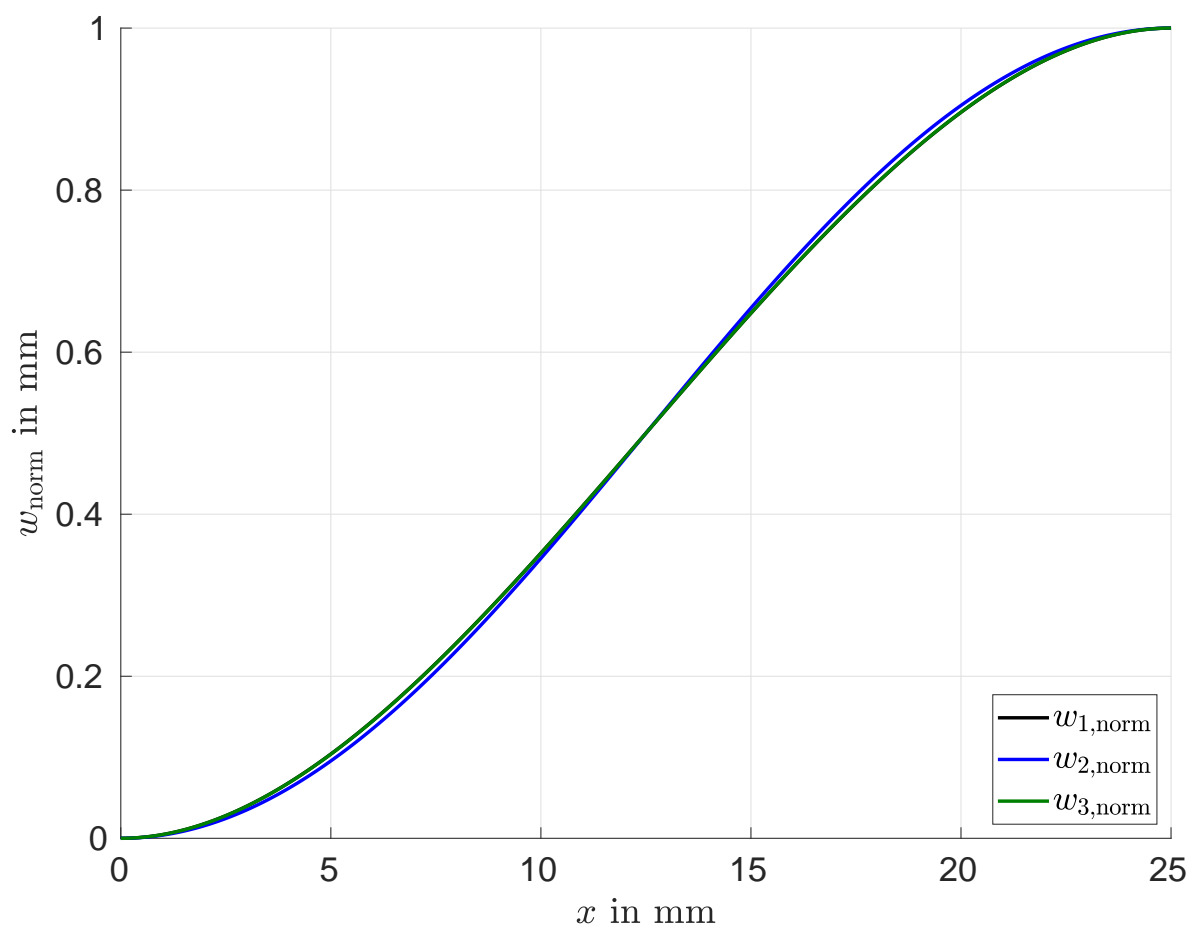

Abb. 3.3 - Darstellung der normierten Verschiebungsverläufe unter Ausnutzung der Symmetrie (Simulationsergebnisse, die Kurven schwarz und grün fallen zusammen)

folgende Form an:

$$
w_{1, \mathrm{norm}}=\frac{w_{1}(x)}{w_{1, \max }}=96\left[\frac{1}{8}\left(\frac{x}{l}\right)^{2}-\frac{1}{6}\left(\frac{x}{l}\right)^{3}\right]
$$




$$
\begin{aligned}
& w_{2, \text { norm }}=\frac{w_{2}(x)}{w_{2, \max }}=\frac{1}{2}\left[1-\cos \left(2 \pi \frac{x}{l}\right)\right] \\
& w_{3, \text { norm }}=\frac{w_{3}(x)}{w_{3, \max }}=\frac{(1-\cos (\lambda l / 2))(1-\cos (\lambda x))+\sin (\lambda l / 2)(\sin (\lambda x)-\lambda x)}{2(1-\cos (\lambda l / 2))-\lambda l / 2 \sin (\lambda l / 2)} .
\end{aligned}
$$

In Abbildung 3.3 sind alle drei normierten Verläufe dargestellt. Obwohl die Biegelinien mathematisch gesehen sehr unterschiedliche Formen haben, zeichnen sie sich durch eine große Ähnlichkeit aus. Alle drei weichen nur sehr geringfügig voneinander ab. Weiterhin kann festgestellt werden, dass die normierten Verläufe nicht von der Störkraft $S$ abhängig sind. Es sei an dieser Stelle aber nochmal ausdrücklich darauf hingewiesen, dass die hier hergeleiteten Gleichungen nur bis zum Bereich des Knickens angewendet werden dürfen. Um das Nachbeulverhalten zu untersuchen, wird auf die Arbeit von Virgin (2007) verwiesen.

\subsubsection{Eigenfrequenzanalyse am Euler-Bernoulli-Balken}

In diesem Abschnitt erfolgt die Eigenfrequenzanalyse am Beispiel eines Euler-BernoulliBalkens. Dabei soll untersucht werden, ob sich mit Hilfe von Eigenfrequenzen Aussagen über das Stabilitätsverhalten von schlanken Strukturen treffen lassen. Aus den analytischen Lösungen sollen wichtige Erkenntnisse abgeleitet werden, wie sie später für die numerische Umsetzung benötigt werden. Die hier hergeleiteten Lösungen dienen der quantitativen Kontrolle und erlauben ein besseres Verständnis bezüglich Knicksicherheit und Stabilität. Für die Eigenfrequenzanalyse eines Euler-Bernoulli-Balkens wird die Differentialgleichung der freien Balkenschwingung herangezogen, siehe Gleichung (3-10) mit $E I=$ konst.:

$$
E I w^{\prime \prime \prime \prime}(x, t)+\rho A \ddot{w}(x, t)+F w^{\prime \prime}(x, t)=0 .
$$

Solche partiellen Differentialgleichungen lassen sich in der Regel mit Hilfe eines Separationsansatzes oder eines Produktansatzes, der eine Trennung der Zeit- und Ortsfunktion vornimmt, lösen (vgl. Dresig u. a. 2007, Gross u.a. 2007). Der hier verwendete Produktansatz mit seiner örtlichen und seiner zeitlichen Ableitung lautet:

$$
\begin{aligned}
w(x, t) & =W(x) \cos (\omega t-\alpha) \\
w^{\prime \prime}(x, t) & =W^{\prime \prime}(x) \cos (\omega t-\alpha) \\
w^{\prime \prime \prime \prime}(x, t) & =W^{\prime \prime \prime \prime}(x) \cos (\omega t-\alpha) \\
\ddot{w}(x, t) & =-W(x) \omega^{2} \cos (\omega t-\alpha) .
\end{aligned}
$$

Setzt man nun die verschiedenen Ableitungen des Produktansatzes in Gleichung (3-10) ein, so lässt sich der zeitabhängige Term: $\cos (\omega t-\alpha)$ wie folgt separieren:

$$
\left[E I W^{\prime \prime \prime \prime}(x)-\rho A \omega^{2} W(x)+F W^{\prime \prime}(x)\right] \cos (\omega t-\alpha)=0 .
$$


Da die Gleichung (3-23) für beliebige zeitabhängige Terme erfüllt sein soll, wird gefordert, dass der Klammerausdruck Null sein muss. Es entsteht eine gewöhnliche Differentialgleichung vierter Ordnung in der Form:

$$
E I W^{\prime \prime \prime \prime}(x)-\rho A \omega^{2} W(x)+F W^{\prime \prime}(x)=0 .
$$

Solche gewöhnlichen Differentialgleichungen können mit Ansätzen des Typs: $W(x)=$ $e^{\lambda x}$ gelöst werden:

$$
\left(E I \lambda^{4}-\rho A \omega^{2}+F \lambda^{2}\right) e^{\lambda x}=0
$$

Die charakteristische Gleichung mit der Substitution $\kappa^{4}=\omega^{2} \frac{\rho A}{E I}$ lautet dabei:

$$
\lambda^{4}-\kappa^{4}+\frac{F}{E I} \lambda^{2}=0
$$

Mit Hilfe von Additionstheoremen $\left(\cosh (\lambda x) \pm \sinh (\lambda x)=e^{ \pm \lambda x}, \cos (\lambda x) \pm i \sin (\lambda x)=\right.$ $\left.e^{ \pm i \lambda x}\right)$ können die Fundamentallösungen der $e$-Funktion in einer Reihe aus Sinus- und Cosinus-Anteilen dargestellt werden. Die allgemeine Lösung nimmt damit folgende Form an (vgl. Gross u. a. 2007):

$$
w(x)=C_{1} \sinh \left(\lambda_{1} x\right)+C_{2} \cosh \left(\lambda_{1} x\right)+C_{3} \sin \left(\lambda_{2} x\right)+C_{4} \cos \left(\lambda_{2} x\right) .
$$

Die Ortskoordinate $x$ des Euler-Bernoulli-Balkens wird über die Variable $\zeta=x / l$ normiert (mit: $\zeta \in[0,1]$ ), um mit dimensionslosen Nullstellen $M$ und $N$ arbeiten zu können:

$$
\begin{aligned}
& M=\lambda_{1} l=l\left[-\frac{1}{2} \frac{F}{E I}+\left[\frac{1}{4}\left(\frac{F}{E I}\right)^{2}+\kappa^{4}\right]^{\frac{1}{2}}\right]^{\frac{1}{2}} \\
& N=\lambda_{2} l=l\left[\frac{1}{2} \frac{F}{E I}+\left[\frac{1}{4}\left(\frac{F}{E I}\right)^{2}+\kappa^{4}\right]^{\frac{1}{2}}\right]^{\frac{1}{2}} .
\end{aligned}
$$

Gleichung (3-27) nimmt dabei folgende Form an:

$$
w(\zeta l)=C_{1} \sinh (M \zeta)+C_{2} \cosh (M \zeta)+C_{3} \sin (N \zeta)+C_{4} \cos (N \zeta)
$$

Die Randbedingungen werden wie in den vorherigen Abschnitten entsprechend dem EulerIV Knickfall gewählt:

$$
\begin{gathered}
w(\zeta l=0)=0, \quad w^{\prime}(\zeta l=0)=0 \\
w(\zeta l=l)=0, \quad w^{\prime}(\zeta l=l)=0 .
\end{gathered}
$$


Zur Bestimmung der unbekannten Konstanten wird ein Gleichungssystem der Form $[A][x]=[b]$ erstellt und nach $[x]=[A]^{-1}[b]$ gelöst:

$$
\left[\begin{array}{cccc}
0 & 1 & 0 & 1 \\
M & 0 & N & 0 \\
\sinh (M) & \cosh (M) & \sin (N) & \cos (N) \\
M \cosh (M) & M \sinh (M) & N \cos (N) & -N \sin (N)
\end{array}\right]\left[\begin{array}{l}
C_{1} \\
C_{2} \\
C_{3} \\
C_{4}
\end{array}\right]=\left[\begin{array}{l}
0 \\
0 \\
0 \\
0
\end{array}\right]
$$

Aus der Determinante von $[A]$ lässt sich eine Entwicklungsgleichung $g(\omega)$ für die Berechnung der Eigenfrequenz $\omega$ bestimmen:

$$
\begin{aligned}
g(\omega):=\operatorname{det}[A] & =\frac{1}{2} e^{-M(\omega)}\left[\left(M^{2}(\omega) \sin (N(\omega))-2 M(\omega) N(\omega) \cos (N(\omega))\right.\right. \\
& \left.-N^{2}(\omega) \sin (N(\omega))\right) e^{2 M(\omega)}+4 M(\omega) e^{M(\omega)} N(\omega)-M^{2}(\omega) \sin (N(\omega)) \\
& \left.-2 M(\omega) N(\omega) \cos (N(\omega))+N^{2}(\omega) \sin (N(\omega))\right] .
\end{aligned}
$$

Mit Hilfe des Newton-Verfahrens kann die niedrigste Eigenfrequenz $\omega$ (Grundfrequenz) iterativ wie folgt ermittelt werden:

$$
\omega_{n+1}=\omega_{n}-\frac{g(\omega)}{g^{\prime}(\omega)} .
$$

In Abbildung 3.4 ist der absolute Eigenfrequenzverlauf des Euler-Bernoulli-Balkens für eine gewählte Länge von $l=50 \mathrm{~mm}$ dargestellt. Für diese Länge ergibt sich ein Instabilitätspunkt $(\omega=0)$ bei ca. $22 \%$ Stauchung. Das bedeutet, dass die Steifigkeitsmatrix des Systems hier singulär wird und die Struktur knickt. Um die hergeleitete und berechnete Lösung der Balken Differentialgleichung zu überprüfen, wird weiterhin das Verfahren von Hamilton verwendet (vgl. Gross u. a. 2007, Hagedorn \& DasGupta 2007). Bei dieser Methode erfolgt die Lösung über das Aufstellen eines zeitlichen Integrals über die sogenannte Lagrangefunktion $L=T-V$ (Differenz zwischen kinetischer Energie $T$ und potentieller Energie $V$ ) und der virtuellen Arbeit $\delta W^{\text {nc }}$ (hier: $\delta W^{\text {nc }}=0$ ):

$$
\begin{array}{r}
\delta \int_{t_{0}}^{t_{1}}(T-V) \mathrm{d} t+\int_{t_{0}}^{t_{1}} \delta W^{\mathrm{nc}} \mathrm{d} t=0 \\
\delta \int_{t_{0}}^{t_{1}} \int_{0}^{l}\left[\frac{1}{2} \rho A \dot{w}^{2}(x, t)-\frac{1}{2} E I w^{\prime 2}(x, t)+\frac{1}{2} F w^{\prime 2}(x, t)\right] \mathrm{d} x \mathrm{~d} t=0 .
\end{array}
$$

Über die Variation der Gleichung (3-33) und der Verwendung eines Produktansatzes: $w(x, t)=W(x) T_{w}(t)$, kann eine Gleichung zur Bestimmung von $\omega$ hergeleitet werden 
(vgl. Gross u. a. 2007, Hagedorn \& DasGupta 2007):

$$
\omega^{2}=\frac{\int_{0}^{l}\left[E I W^{\prime \prime 2}(x)-F W^{\prime 2}(x)\right] \mathrm{d} x}{\int_{0}^{l}\left[\rho A W^{2}(x)\right] \mathrm{d} x} .
$$

Die Formfunktion $W(x)$ kann entsprechend Gleichung (3-15) gewählt werden. Die gute Übereinstimmung zwischen der Lösung der Balken-Differentialgleichung (rot) und der Lösung resultierend dem Rayleigh-Ritz-Prinzip (blau) kann der Abbildung 3.4 entnommen werden. Um unabhängig von der gewählten Materialsteifigkeit (hier: dem E-Modul) zu

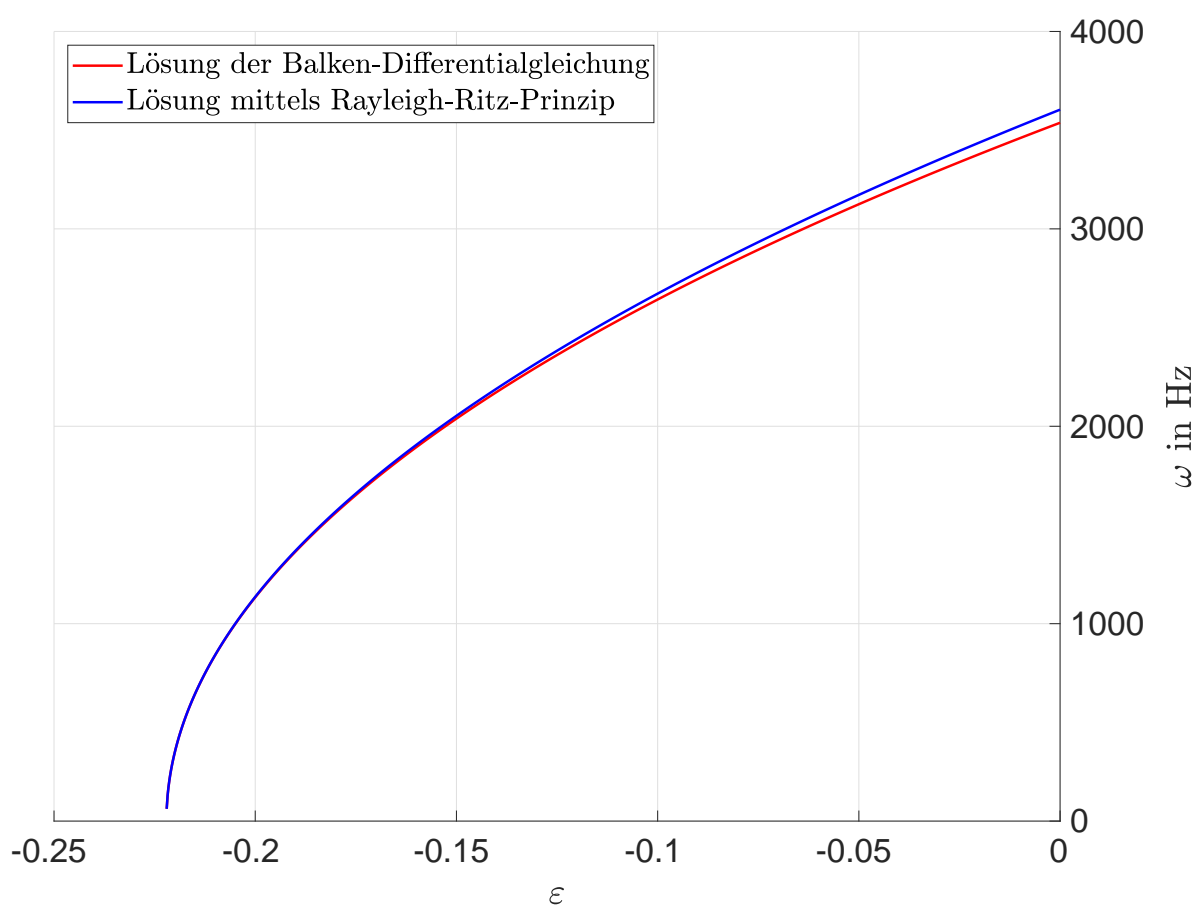

Abb. 3.4 - Darstellung der absoluten Eigenfrequenzverläufe eines EulerBernoulli-Balkens über der Dehnung $\varepsilon$ aufgrund der Normalkräfte (Simulationsergebnisse)

sein, wird der Eigenfrequenzverlauf mit $\omega_{0}$ normiert, siehe Abbildung 3.5. Aus diesen Eigenfrequenzanalysen lassen sich eine Reihe interessanter Schlussfolgerungen ziehen, die im Folgenden zusammengefasst werden sollen.

\section{Zusammenfassung der elastischen Eigenfrequenzanalysen:}

Mit Hilfe des Eigenfrequenzverlaufes lassen sich Aussagen zum Stabilitätsverhalten von schlanken Strukturen treffen. Beispielsweise beschreibt der Nulldurchgang des Eigenfrequenzverlaufes den Punkt der Instabilität, wo die Struktur zu knicken beginnt. Anders ausgedrückt, die Steifigkeitsmatrix des elastischen Systems wird an diesem Punkt singulär. Der Verlauf der Eigenfrequenz kann somit als ein Maß für die Sicherheit gegenüber 


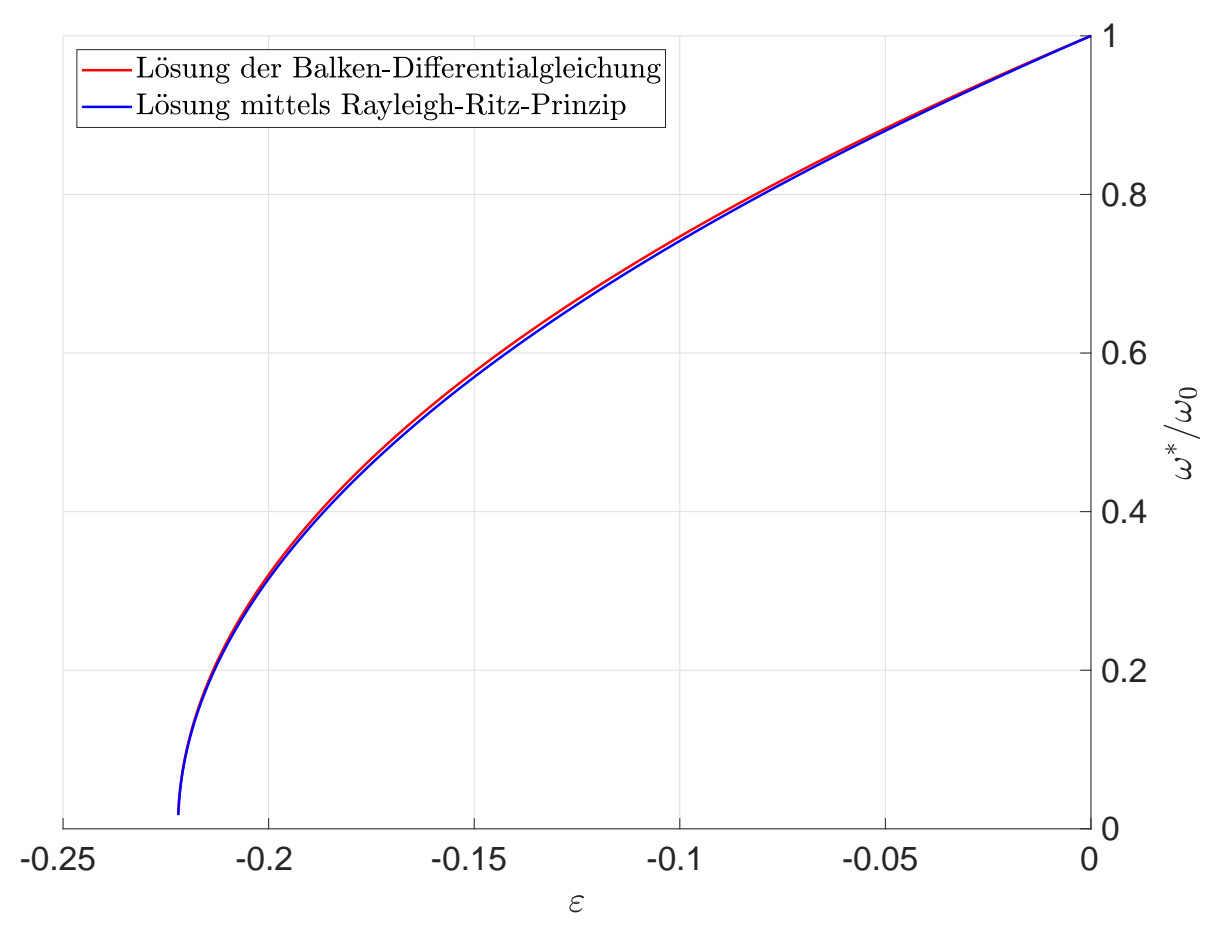

Abb. 3.5 - Darstellung der normierten Eigenfrequenzverläufe eines EulerBernoulli-Balkens über der Dehnung $\varepsilon$ aufgrund der Normalkräfte (Simulationsergebnisse, vgl. Abbildung 3.4

Ausknicken angesehen werden. Gelingt es nun den Eigenfrequenzverlauf in der Weise zu manipulieren, dass dieser keinen Nulldurchgang erfährt, so erhält man als Ergebnis endliche Knickstabilität. Ziel sollte es daher sein, den Eigenfrequenzverlauf in der Weise zu modifizieren, dass ein frühes Ausknicken (momentan bei $22 \%$ Stauchung) verhindert wird und die Struktur noch genügend Reserve gegenüber Knicken besitzt. Weiterhin ist festzuhalten, dass die Knicksicherheit bzw. -stabilität entscheidend von der Knicklänge und dem Flächenträgheitsmoment abhängt.

\subsubsection{Erweiterung der Eigenfrequenzanalyse für viskoelastische Strukturen}

Im Folgenden soll die Eigenfrequenzanalyse für einen viskoelastischen Euler-BernoulliBalken durchgeführt werden. Hierbei soll insbesondere untersucht werden, welchen Einfluss viskoelastische Effekte auf das Stabilitätsverhalten von Balken ausüben. Eine elegante Methode zur Berücksichtigung der Dämpfung ist das Rayleigh-Ritz-Prinzip. Die Energien und die virtuelle Arbeit lauten dabei wie folgt (Verwendung des Zener-Modells):

$$
\begin{aligned}
T & =\int_{0}^{l} \frac{1}{2} \rho A \dot{w}^{2}(x, t) \mathrm{d} x \\
V & =\int_{0}^{l}\left[\frac{1}{2} E_{\infty} I w^{\prime \prime 2}(x, t)+\frac{1}{2} E_{1} I\left(w^{\prime \prime}(x, t)-v^{\prime \prime}(x, t)\right)^{2}-\frac{1}{2} F w^{\prime 2}(x, t)\right] \mathrm{d} x
\end{aligned}
$$




$$
\delta W^{\mathrm{nc}}=-\int_{0}^{l} \eta I \dot{v}^{\prime \prime}(x, t) \delta v^{\prime \prime}(x, t) \mathrm{d} x .
$$

Die innere Dehnung $\gamma$ des Zener-Modells (vgl. Abbildung 3.6) wird kinematisch genauso behandelt wie die Gesamtdehnung: $\varepsilon=-w^{\prime \prime}(x, t) z \rightarrow \gamma=-v^{\prime \prime}(x, t) z$. Es sei an dieser Stelle darauf hingewiesen, dass das hier aufgestellte Modell nur für eine konstante Druckkraft $F=$ konst. anwendbar ist. Für eine zeitlich veränderliche Druckkraft $F(t)$ müsste eine Längsdehn-Biegeschwingung betrachtet werden, was zu einer gekoppelten partiellen Differentialgleichung mit zwei Verschiebungen $u(x, t), w(x, t)$ und zwei inneren Variablen $s(x, t), v(x, t)$ führen würde (vgl. Hagedorn \& DasGupta 2007, Virgin 2007). Da diese Modellerweiterung den Rahmen der hier durchzuführenden Untersuchungen sprengen würde, soll mit dem statischen Grenzfall $F=$ konst. weiter gearbeitet werden. Über die Gleichung (3-32) erhält man für die oben beschriebenen Terme folgenden Ausdruck:

$$
\begin{array}{r}
\delta \int_{t_{0}}^{t_{1}} \int_{0}^{l} \frac{1}{2}\left[\rho A \dot{w}^{2}(x, t)-E_{\infty} I w^{\prime \prime 2}(x, t)-E_{1} I\left(w^{\prime \prime}(x, t)-v^{\prime \prime}(x, t)\right)^{2}+F w^{\prime 2}(x, t)\right] \mathrm{d} x \mathrm{~d} t \\
-\int_{t_{0}}^{t_{1}} \int_{0}^{l} \eta I \dot{v}^{\prime \prime}(x, t) \delta v^{\prime \prime}(x, t) \mathrm{d} x \mathrm{~d} t=0 .
\end{array}
$$

Mit Hilfe der Produktansätze: $w(x, t)=W(x) T_{w}(t) ; v(x, t)=V(x) T_{v}(t)$ und der Ausführung der Variation entsteht folgender Zusammenhang:

$$
\begin{aligned}
\int_{t_{0}}^{t_{1}} \int_{0}^{l}[ & -\rho A W^{2}(x) \ddot{T}_{w}(t) \delta T_{w}(t)-E_{\infty} I W^{\prime \prime 2}(x) T_{w}(t) \delta T_{w}(t) \\
& -E_{1} I\left(W^{\prime \prime}(x) T_{w}(t)-V^{\prime \prime}(x) T_{v}(t)\right)\left(W^{\prime \prime}(x) \delta T_{w}(t)-V^{\prime \prime}(x) \delta T_{v}(t)\right) \\
& \left.+F W^{\prime 2}(x) T_{w}(t) \delta T_{w}(t)-\eta I V^{\prime \prime 2}(x) \dot{T}_{v}(t) \delta T_{v}(t)\right] \mathrm{d} x \mathrm{~d} t=0 .
\end{aligned}
$$

Über einen Koeffizientenvergleich erhält man die Bewegungsgleichung:

$$
[\mathbf{M}]\left[\begin{array}{c}
\ddot{T}_{w} \\
\ddot{T}_{v}
\end{array}\right]+[\mathbf{D}]\left[\begin{array}{c}
\dot{T}_{w} \\
\dot{T}_{v}
\end{array}\right]+[\mathbf{K}]\left[\begin{array}{c}
T_{w} \\
T_{v}
\end{array}\right]=[\mathbf{0}]
$$

mit den Matrixeinträgen:

$$
[\mathbf{M}]=\left[\begin{array}{cc}
-\rho A \int_{0}^{l} W^{2}(x) \mathrm{d} x & 0 \\
0 & 0
\end{array}\right] ; \quad[\mathbf{D}]=\left[\begin{array}{cc}
0 & 0 \\
0 & -\eta I \int_{0}^{l} V^{\prime \prime 2}(x) \mathrm{d} x
\end{array}\right] ;
$$




$$
[\mathbf{K}]=\left[\begin{array}{cc}
-\left(E_{\infty}+E_{1}\right) I \int_{0}^{l} W^{\prime \prime 2}(x) \mathrm{d} x+F \int_{0}^{l} W^{\prime 2}(x) \mathrm{d} x & E_{1} I \int_{0}^{l} W^{\prime \prime}(x) V^{\prime \prime}(x) \mathrm{d} x \\
E_{1} I \int_{0}^{l} W^{\prime \prime}(x) V^{\prime \prime}(x) \mathrm{d} x & -E_{1} I \int_{0}^{l} V^{\prime \prime 2}(x) \mathrm{d} x
\end{array}\right] .
$$

Zur besseren Lösbarkeit wird die Bewegungsgleichung zweiter Ordnung (3-38) in ein System erster Ordnung überführt. Das Differentialgleichungssystem lautet dabei wie folgt:

$$
z=\left[\begin{array}{c}
q \\
\dot{q}
\end{array}\right] ; \quad \dot{z}=\left[\begin{array}{c}
\dot{q} \\
\ddot{q}
\end{array}\right] \Rightarrow\left[\begin{array}{cc}
\mathbf{I} & \mathbf{0} \\
\mathbf{0} & \mathbf{M}
\end{array}\right]\left[\begin{array}{c}
\dot{q} \\
\ddot{q}
\end{array}\right]=\left[\begin{array}{cc}
\mathbf{0} & \mathbf{I} \\
-\mathbf{K} & -\mathbf{D}
\end{array}\right]\left[\begin{array}{c}
q \\
\dot{q}
\end{array}\right] \Rightarrow B \dot{z}=A z
$$

Mit dem Exponentialansatz: $z=\hat{z} e^{\lambda t}$ erhält man ein generalisiertes Eigenwertproblem:

$$
A \hat{z}=\lambda B \hat{z}
$$

Die Eigenwerte $\lambda_{1,2}$ ergeben zusammen ein konjugiert komplexes Eigenwertpaar (schwingende Lösung) und $\lambda_{3}$ beschreibt den reelen Eigenwert (auf- oder abklingende Lösung). Der vierte Eigenwert ist unendlich, weil für die interne Variable $\gamma$ eine massefreie Hilfskoordinate eingeführt wurde. Zur Überprüfung der korrekten Implementierung des viskoelastischen Balkens werden in Abbildung 3.6 die zwei Grenzfälle des Zener-Modells (weicher und quasi starrer Dämpfer) mit den jeweiligen elastischen Lösungen verglichen. Aus der Abbildung wird deutlich, dass eine sehr niedrige Viskosität (unterer Grenzfall):

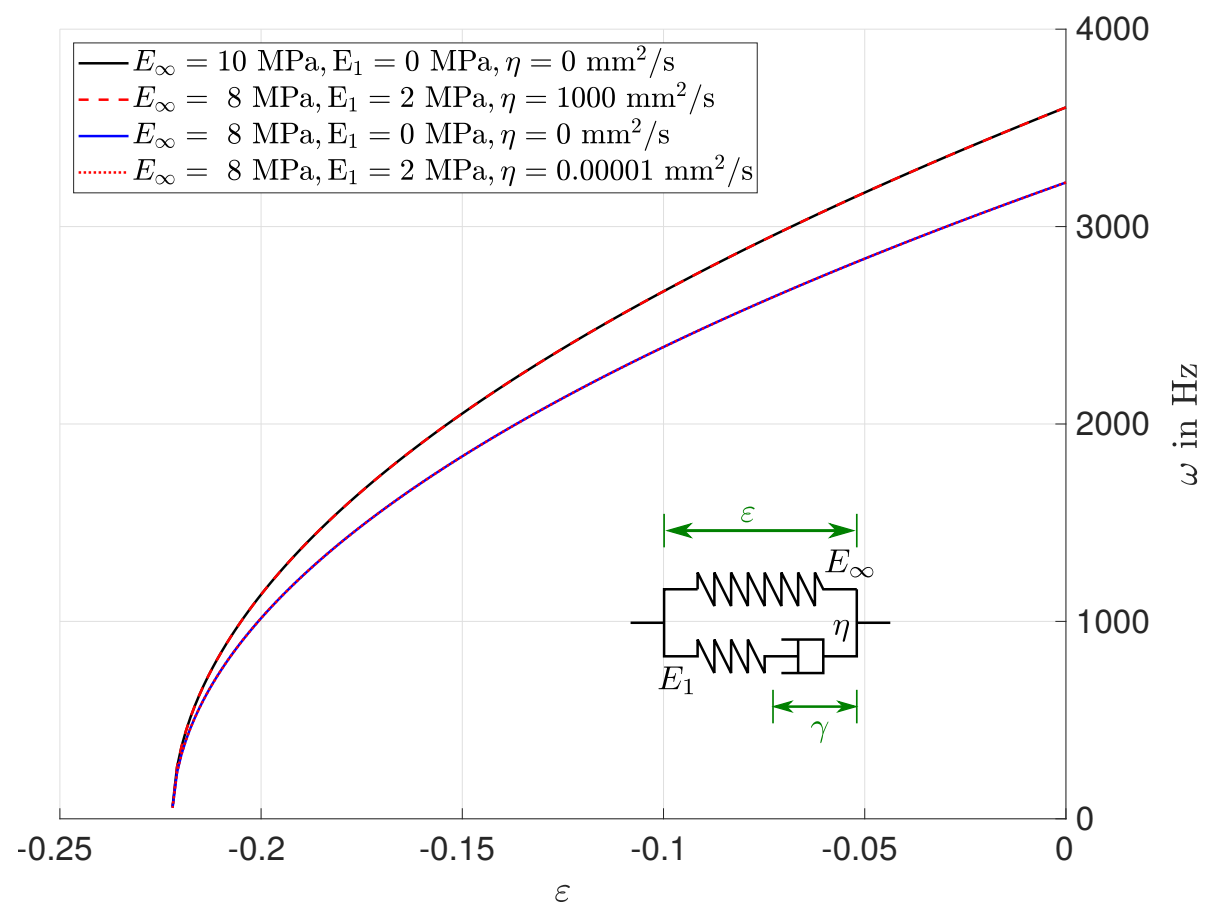

Abb. 3.6 - Darstellung der absoluten Eigenfrequenzverläufe eines viskoelastischen Euler-Bernoulli-Balkens zur Überprüfung der zwei Grenzfälle (Simulationsergebnisse, die ersten beiden Kurven und die letzten beiden Kurven fallen zusammen) 
$E_{\infty}=8 \mathrm{MPa}, E_{1}=2 \mathrm{MPa}, \eta=0.00001 \mathrm{~mm}^{2} / \mathrm{s}$ zu dem gleichen Ergebnis führt wie die rein elastische Lösung mit: $E_{\infty}=8 \mathrm{MPa}, E_{1}=0 \mathrm{MPa}, \eta=0 \mathrm{~mm}^{2} / \mathrm{s}$. Das bedeutet, dass die niedrige Viskosität des Dämpfers die Feder $E_{1}$ komplett neutralisiert. Als nächstes soll der Grenzfall eines quasi starren Dämpfers näher untersucht werden. Hier zeigt sich, dass das Zener-Modell mit einer sehr hohen Viskosität (oberer Grenzfall): $E_{\infty}=8 \mathrm{MPa}, E_{1}=2 \mathrm{MPa}, \eta=1000 \mathrm{~mm}^{2} / \mathrm{s}$ zu dem gleichen Ergebnis führt wie die rein elastische Lösung: $E_{\infty}=10 \mathrm{MPa}, E_{1}=0 \mathrm{MPa}, \eta=0 \mathrm{~mm}^{2} / \mathrm{s}$. Die Steifigkeit der beiden Federn wird hier aufsummiert (Prinzip einer Parallelschaltung).

Im Weiteren wird die Viskosität zwischen den Grenzbereichen variiert. Dabei soll der Einfluss auf den Eigenfrequenzverlauf näher untersucht werden. In der Abbildung 3.7 sind dazu einige ausgewählte Größen dargestellt. Anhand der Abbildung wird deutlich, dass

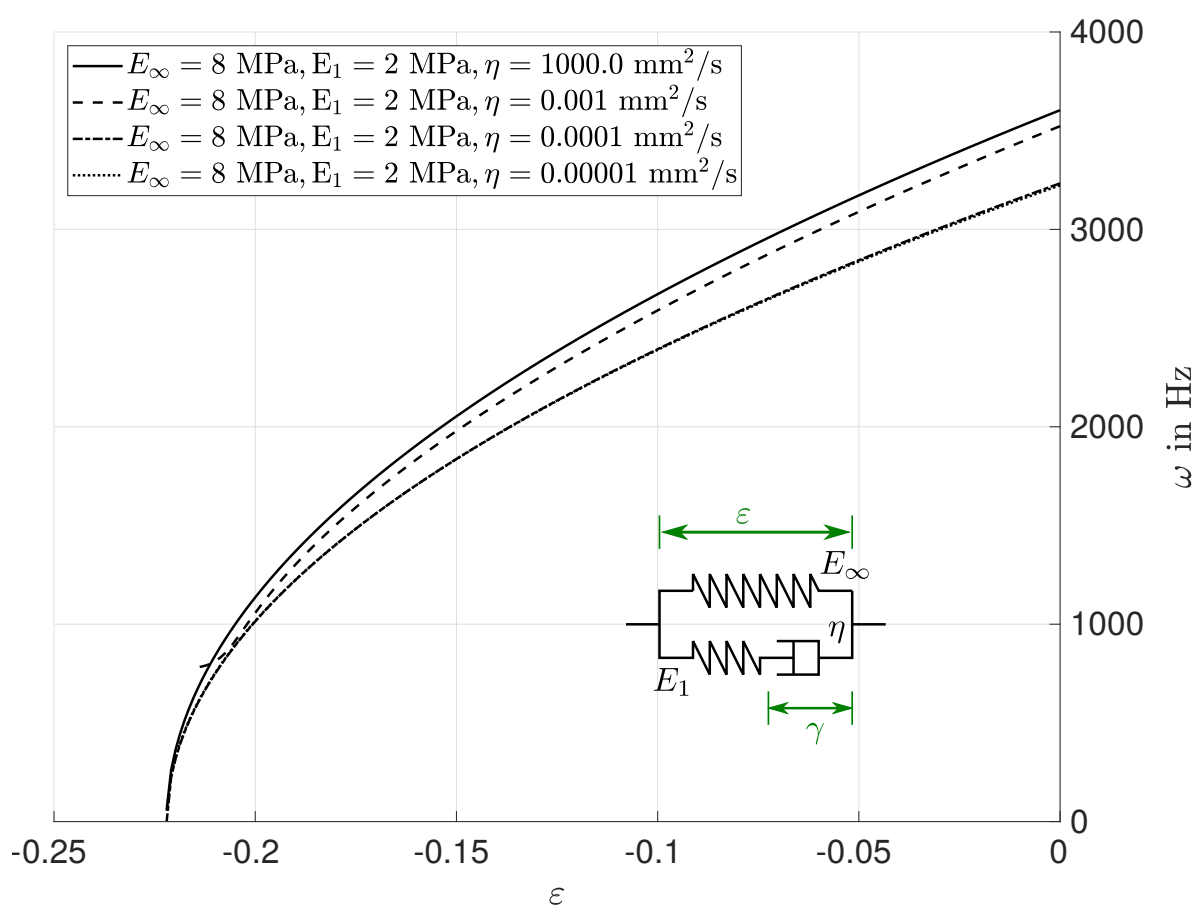

Abb. 3.7 - Darstellung der absoluten Eigenfrequenzverläufe eines viskoelastischen Euler-Bernoulli-Balkens unter Variation der Viskosität (Simulationsergebnisse, vgl. Abbildung 3.6 die letzten beiden Kurven fallen zusammen)

die Viskosität in direkter Weise Einfluss auf die Höhe der Eigenfrequenz des unbelasteten Stabes nimmt. Weiterhin ist zu erkennen, dass bei einer Viskosität von $\eta=0.001 \mathrm{~mm}^{2} / \mathrm{s}$ ein detektierbarer Unterschied zu dem oberen Grenzfall auftritt. Im Vergleich dazu führt eine Viskosität von $\eta=0.0001 \mathrm{~mm}^{2} / \mathrm{s}$ zu einem starken Abfall der Eigenfrequenz nahe des unteren Grenzfalls. Als nächstes soll auf den Knickpunkt näher eingegangen werden. Hier wird deutlich, dass sich für sehr hohe und sehr niedrige Viskositäten der gleiche Instabilitätspunkt wie bei der elastischen Lösung ergibt. Für Viskositäten nahe $\eta=0.001 \mathrm{~mm}^{2} / \mathrm{s}$ ist gegen Ende der Beanspruchung ein leicht abweichender Verlauf zu erkennen, der über die vollständige Analyse aller Eigenwerte zu erklären ist. Für geplante FE-Berechnungen 
kann festgehalten werden, dass die elastische Lösung zur Bestimmung der kritischen Stauchung, bei der Knicken auftritt, völlig ausreichend ist.

\subsection{FE gestützte Entwicklung eines Zug-/Druckprobekörpers}

\subsubsection{Grundidee des Halterungsdesigns}

Im vorherigen Abschnitt wurde bereits gezeigt, dass sich mit Hilfe von Eigenfrequenzen Aussagen zum Stabilitätsverhalten von schlanken Strukturen treffen lassen. In diesem Abschnitt soll die Untersuchung auf numerischem Wege mittels FeM fortgesetzt werden. Im Gegensatz zu analytischen Untersuchungen lassen sich hier beliebige komplexe Strukturen berechnen und analysieren. Bevor allerdings mit der Untersuchung dieser Strukturen begonnen werden kann, soll zunächst der Eigenfrequenzverlauf der Abbildung 3.5 auf numerischem Wege überprüft werden. Als Materialmodell findet hier das hyperelastische Stoffgesetz nach Mooney-Rivlin Anwendung (vgl. dazu Abschnitt 2.1.4 und Tabelle 3.1). In FE-Programmen können Eigenfrequenzen und Eigenschwingungsformen einer beliebi-

\begin{tabular}{|c|c|c|}
\hline$c_{10}$ in $\mathrm{MPa}$ & $c_{01}$ in $\mathrm{MPa}$ & $K$ in $\mathrm{MPa}$ \\
\hline 1.0 & 0.1 & 1000 \\
\hline
\end{tabular}

Tabelle 3.1 - Materialparameter für Mooney-Rivlin (vgl. Naumann 2010)

gen Struktur mittels Modalanalyse berechnet werden. Um den Effekt der Spannungsversteifung mit zu berücksichtigen, ist eine vorgespannte Modalanalyse nötig (vgl. Stelzmann u. a. 2002). In der Gleichung (3-41) ist der mathematische Zusammenhang zur Ermittlung der Eigenfrequenzen dargestellt (vgl. Dresig u. a. 2007):

$$
\left(\left[\mathbf{K}_{\mathrm{L}}\right]+\left[\mathbf{K}_{\mathrm{NL}}\right]-\omega_{j}^{2}(u)[\mathbf{M}]\right)\left[\varphi_{j}\right]=[0] .
$$

Darin beschreibt $\left[\mathbf{K}_{\mathrm{L}}\right]$ den linearen und $\left[\mathbf{K}_{\mathrm{NL}}\right]$ den nichtlinearen Anteil der Steifigkeitsmatrix. $[\mathbf{M}]$ gibt die Massenmatrix an und $\omega_{j}(u)$ die Eigenfrequenz in Abhängigkeit der Belastung $u$. An dieser Formulierung wird auch der intuitive Zugang deutlich, d. h. dass die Berechnung der Nullstelle der Gleichung (3-41) gleichzusetzen ist, mit der Suche nach dem Punkt der Instabilität. Diese Überlegung führt zu der Schlussfolgerung, dass die erste Eigenfrequenz $\omega_{1}$ (niedrigste Eigenfrequenz) für die Stabilitätsuntersuchungen von besonderer Bedeutung ist. In Abbildung 3.8 ist der Eigenfrequenzverlauf für einen Stab der Länge $l_{0}=50 \mathrm{~mm}$ mit einem Durchmesser $d=15 \mathrm{~mm}$ dargestellt (rote Kurve). Für diese Abmessungen erreicht der Eigenfrequenzverlauf einen Nulldurchgang bei $22 \%$ Stauchung. Die Dehnung $\varepsilon$ wird dabei über die Längenänderung $\Delta l$ und die Messlänge $l_{\mathrm{M}}$ bestimmt. Vergleicht man dieses Ergebnis mit der Abbildung 3.5 dann erhält man eine sehr gute Übereinstimmung mit den analytischen Lösungen. Als nächstes wird die Charakterisierung der Eigenfrequenzverläufe für komplexere Strukturen fortgesetzt. Dazu wird 


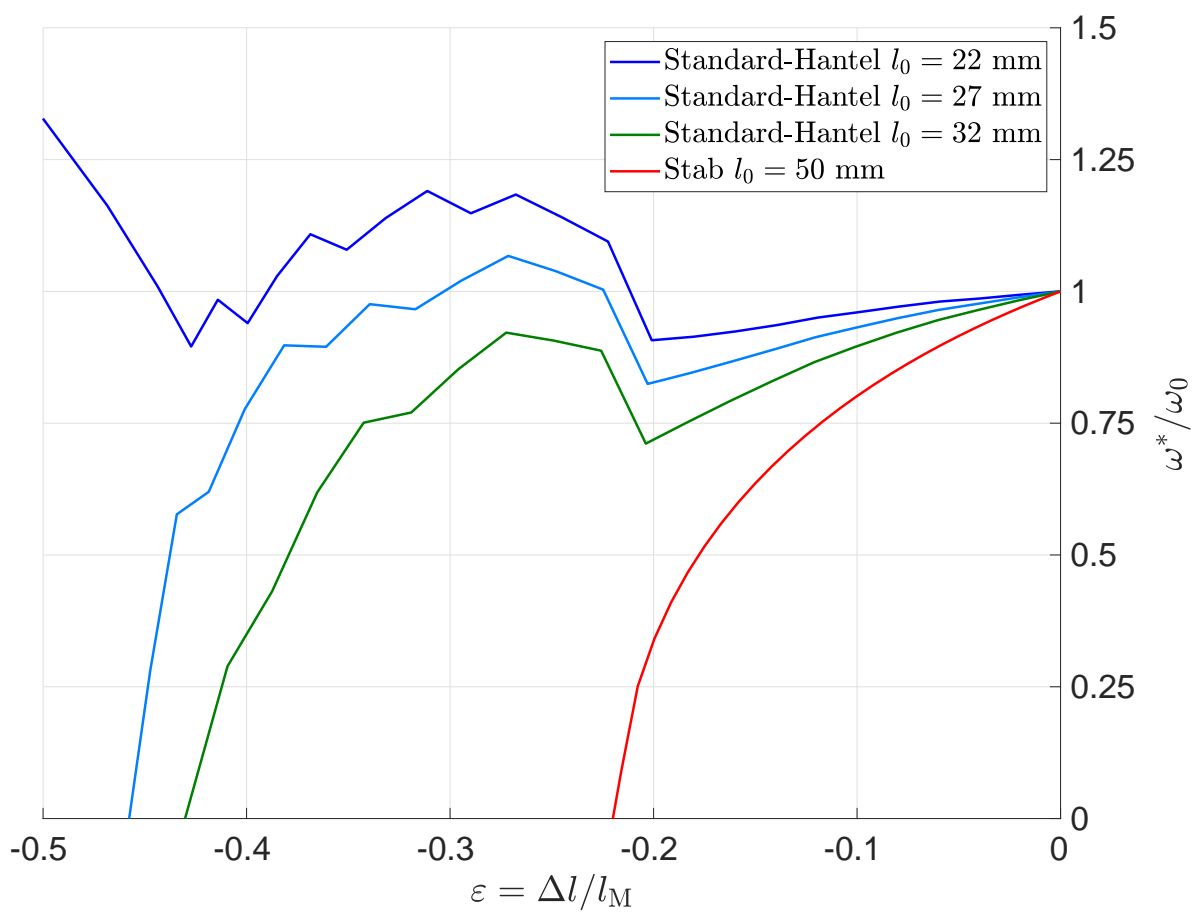

Abb. 3.8 - Modalanalyse zur Untersuchung der Eigenfrequenzverläufe der Standard-Hantel für verschiedene Ausgangslängen (Simulationsergebnisse vgl. Tabelle 3.1. Abbildung in Anlehnung an die Arbeit von Naumann 2010)

der standardmäßige Hantelprobekörper $\left(l_{0}=22 \mathrm{~mm}\right)$ näher untersucht. In Abbildung 1.1 ist der Hantelprobekörper schematisch dargestellt. Aus der Veröffentlichung von Alshuth u. a.(2007) wird ersichtlich, dass der Hantelprobekörper im Messbereich mit zunehmender Stauchung eine inhomogene Belastungsverteilung aufweist. Im Folgenden soll mittels einer vorgespannten Modalanalyse der Eigenfrequenzverlauf der Standard-Hantel $\left(l_{0}=22 \mathrm{~mm}\right)$ mit zugehöriger Halterung berechnet werden. Der Verlauf ist zum besseren Vergleich ebenfalls in Abbildung 3.8 dargestellt (dunkelblaue Kurve). Aus dieser wird ersichtlich, dass die Hantelgeometrie mit zugehöriger Halterung zu keinem Nulldurchgang des Eigenfrequenzverlaufes führt. Das bedeutet, dass der aktuelle Probekörper zu keinem Zeitpunkt knickgefährdet ist bzw. kein Instabilitätspunkt vorhanden ist. Es ist weiterhin zu erkennen, dass bei $20 \%$ Stauchung ein abrupter Anstieg des Eigenfrequenzverlaufes zu beobachten ist. Dieser abrupte Anstieg lässt sich auf den unmittelbaren Kontakt der Hantel mit der Halterungsgeometrie zurückführen. Diese Kontaktierung hat zunächst eine stabilisierende Wirkung auf den Hantelprobekörper (Anstieg des Eigenfrequenzverlaufes), allerdings nimmt dieser Einfluss über 30 \% Stauchung wieder ab. Der Anstieg am Ende kann dadurch erklärt werden, dass der Hantelprobekörper mittlerweile soweit gestaucht ist, dass keine Knickgefahr mehr besteht. Wie in der Veröffentlichung von Alshuth u. a. gezeigt, besitzt die Hantel für diese kurze Abmessung ein sehr inhomogenes Verzerrungsfeld im Messbereich. Um die Homogenität im Messbereich zu erhöhen, empfiehlt sich die Verwendung von längeren Hantelprobekörpern. Der Grundgedanke dabei ist, dass eine Verlängerung des Mittelteils auch zu einem größeren Abstand zwischen Messbereich und zugehöriger 
Halterung führt. Die Analysen werden für die Standard-Hantel mit der Mittelteillänge $l_{0}=27 \mathrm{~mm}$ und $l_{0}=32 \mathrm{~mm}$ erweitert. Aus der Abbildung 3.8 wird ersichtlich, dass schon eine Erhöhung der Mittelteillänge um $5 \mathrm{~mm}$ zu einem starken Abfall des Eigenfrequenzverlaufes führt und ein Nulldurchgang erreicht wird. Aus den Analysen werden zwei wichtige Sachverhalte deutlich. Zum einen ist eine längere Mittelteillänge zur Verbesserung der Homogenität zu empfehlen, was sich wiederum nachteilig auf die Knicksicherheit auswirkt. Zum anderen hat eine kürzere Mittelteillänge eine wesentlich höhere Knicksicherheit mit dafür nachteilig inhomogenem Verzerrungsfeld im Messbereich. Im Rahmen der Arbeit von Naumann (2010) wurde ein Konzept entwickelt, welches es ermöglicht, auf der Grundlage von Eigenfrequenzverläufen neue Halterungsgeometrien zu berechnen.

Im Folgenden soll diese Grundidee des neuen Halterungsdesigns näher vorgestellt werden. In Abbildung 3.9 ist der schematische Ablaufplan zur Entwicklung neuer Halterungsgeometrien dargestellt (vgl. Naumann 2010). Auf der linken Seite der Abbildung 3.9 ist

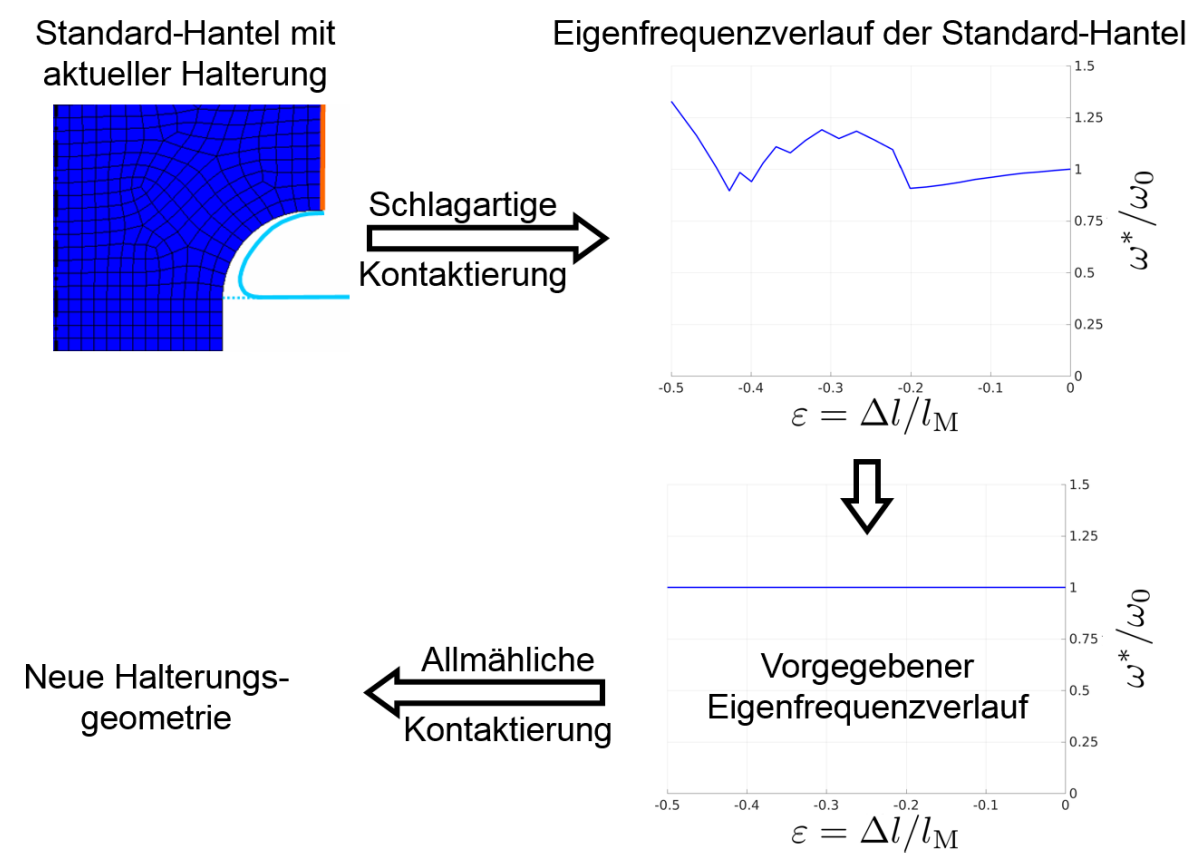

Abb. 3.9 - Grundidee des Halterungsalgorithmus (Simulationsergebnisse vgl. Tabelle 3.1. Abbildung in Anlehnung an die Arbeit von Naumann 2010)

der axialsymmetrische Ausschnitt der Standard-Hantel mit zugehöriger Halterung zu sehen. Bei einer Stauchung erfährt der Hantelprobekörper mit der bisherigen Halterung eine schlagartige Kontaktierung. Das bedeutet, dass der Probekörper nicht allmählich mit der Halterung in Kontakt tritt, sondern sehr abrupt. Mittels vorgespannter Modalanalyse kann der zugehörige Eigenfrequenzverlauf berechnet werden, vgl. dazu Abbildung 3.8 (dunkelblaue Kurve). Die Grundidee besteht darin, den Eigenfrequenzverlauf $\omega^{*}$ vorzugeben und invers dazu die zugehörige Halterungsgeometrie zu berechnen. Diese Methode, die im Rahmen der Arbeit von Naumann (2010) entwickelt worden ist, stellt ein wichtiges 
Werkzeug in der Probekörperentwicklung dar. Beispielsweise kann durch einen konstanten Eigenfrequenzverlauf eine allmähliche Kontaktierung zwischen Probekörper und neuer Halterungsgeometrie sichergestellt werden. Somit kann über die Eigenfrequenzvorgabe $\omega^{*}$ in indirektem Maße Einfluss auf die Knicksicherheit und die Homogenität des Probekörpers genommen werden.

\subsubsection{Algorithmus zur Berechnung von Halterungsgeometrien}

In diesem Abschnitt wird der Algorithmus, wie er für die Berechnung verschiedener Halterungsgeometrien verwendet wird, vorgestellt. Der Algorithmus ist im Rahmen der Arbeit von Naumann (2010) entwickelt worden. Im Folgenden werden die wichtigsten Schritte, die zum Verständnis beitragen sollen, erläutert. In Abbildung 3.10 ist der schematische Ablaufplan des Halterungsalgorithmus dargestellt. Zu Beginn wird mit Hilfe einer Mo-
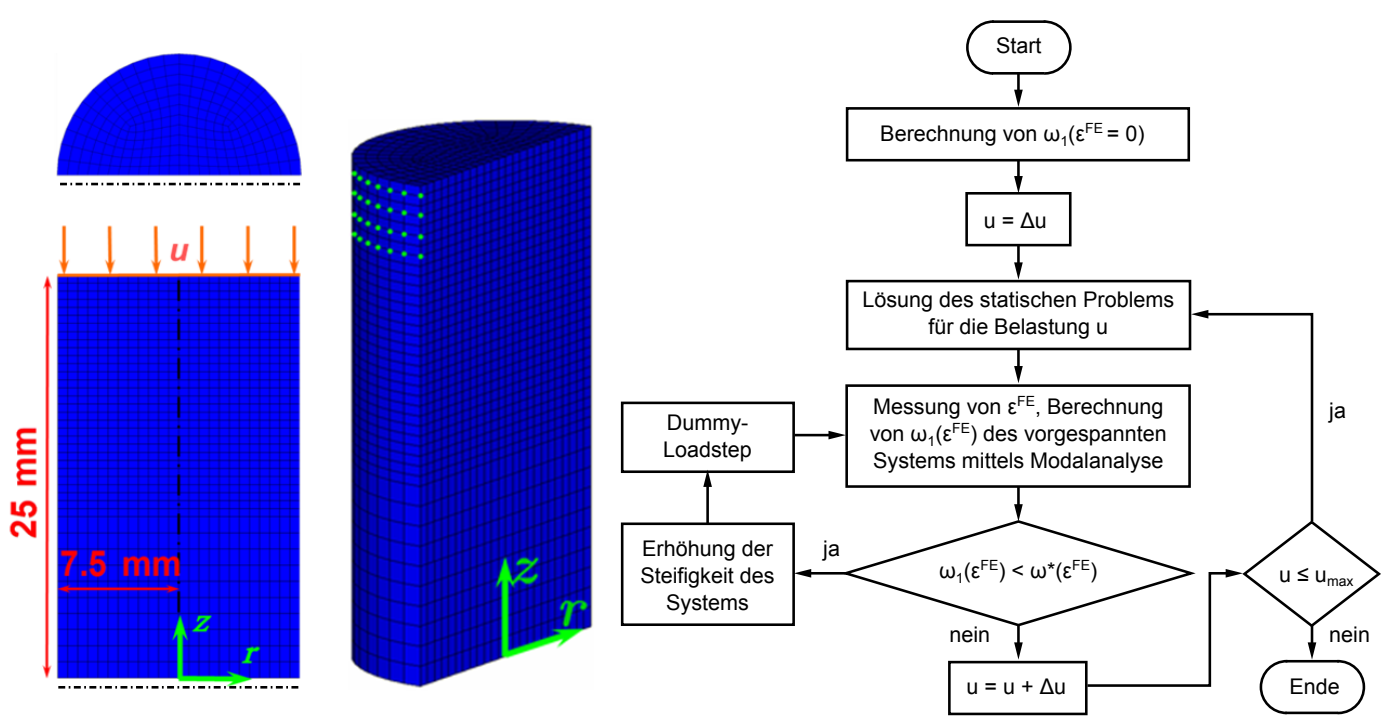

Abb. 3.10 - Algorithmus zur Berechnung von Halterungskonturen (Abbildung aus der Arbeit von Naumann 2010)

dalanalyse die Eigenfrequenz der unbelasteten Struktur berechnet. Im nächsten Schritt wird die Belastung inkrementell erhöht und das statische Problem gelöst. Im Anschluss daran wird für die nun vorgespannte Struktur eine Modalanalyse durchgeführt. Die aktuell berechnete Eigenfrequenz $\omega_{1}$ wird mit der Eigenfrequenzvorgabe $\omega^{*}$ verglichen. Wenn der berechnete Eigenfrequenzverlauf kleiner als der vorgegebene ist, muss die Steifigkeit des Systems erhöht werden. Dies erfolgt in der Fixierung der äußeren Knoten auf der Zylinderstruktur in radialer und tangentialer Richtung. In der Abbildung 3.10 ist dies auf der linken Seite anhand der grün gekennzeichneten Knoten dargestellt. Ist der berechnete Eigenfrequenzverlauf größer als der vorgegebene, kann die Last weiter erhöht werden und der nächste Berechnungsschritt starten. Die Berechnung endet, wenn die maximale Verschiebung erreicht ist. Über diesen Algorithmus kann sukzessive die gesuchte Halterungskontur berechnet werden. Es sei an dieser Stelle darauf hingewiesen, dass sowohl 
die Zylindergeometrie als auch die Belastung selber hinreichend fein diskretisiert sein sollte. Dadurch wird gewährleistet, dass die Halterungskontur sehr genau abgebildet werden kann. Im nächsten Abschnitt soll mit Hilfe des hier vorgestellten Halterungsalgorithmus eine Parameterstudie durchgeführt werden.

\subsubsection{Parameterstudie zur Halterungsberechnung}

Anhand des entwickelten Halterungsalgorithmus soll im Folgenden eine Parameterstudie zur Untersuchung der Sensitivität der Halterungsberechnung durchgeführt werden. Die dafür benötigten Eigenfrequenzvorgaben sollen in der Weise modelliert werden, dass ein möglichst großer Anwendungsbereich abgedeckt wird. Im Weiteren soll überprüft werden, wie gut das Halterungskonzept allgemein funktioniert und wie sensitiv sich die modellierten Eigenfrequenzverläufe auf die Halterungsberechnung auswirken. In Abbildung 3.11 sind die normierten vorgegebenen Eigenfrequenzverläufe $\omega^{*}(\varepsilon) / \omega_{0}$, die zur Berechnung von Halterungsgeometrien benötigt werden, dargestellt. Die schwarzen Zickzackkurven

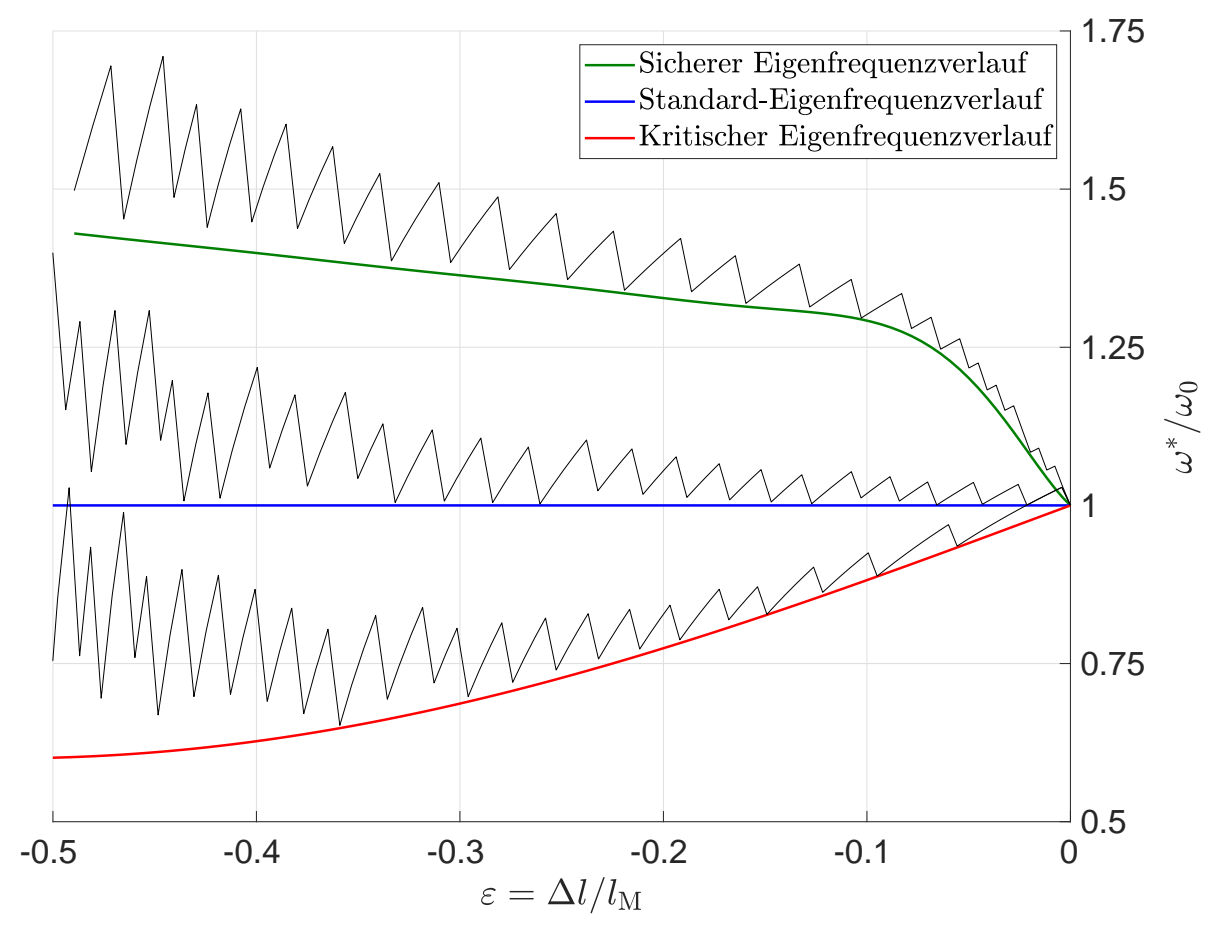

Abb. 3.11 - Parameterstudie zur Eigenfrequenzvorgabe für die Halterungsberechnung (Simulationsergebnisse vgl. Tabelle 3.1)

beschreiben dabei die tatsächlich vom Halterungsalgorithmus berechneten Verläufe. Wird beispielsweise die Eigenfrequenzvorgabe nicht erfüllt, so erfolgt eine weitere Fixierung von äußeren Knoten auf der Zylindergeometrie (vgl. Abbildung 3.10), was zu einem kurzzeitigen Anstieg des Eigenfrequenzverlaufes führt. Die Abweichungen des Zickzackverlaufes von der Eigenfrequenzvorgabe bei größeren Stauchungen lassen sich dadurch erklären, dass die knickgefährdete Länge immer stärker reduziert wird, was eine abnehmende Fixierung von Knoten nach sich zieht. Die Eigenfrequenzmodellierung erfolgte in der Weise, 
dass unterschiedliche Reserven bezüglich Homogenität als auch Stabilität vorausgesetzt sind. Beispielsweise stellt der blaue Eigenfrequenzverlauf, der im Folgenden als StandardEigenfrequenzverlauf bezeichnet wird, eine mittlere Variante zur Halterungsberechnung dar. Bei diesem Eigenfrequenzverlauf wird gefordert, dass die aktuelle Eigenfrequenz niemals die Starteigenfrequenz $\omega^{*}(\varepsilon) / \omega_{0}=1$ unterschreiten darf. Anhand der schwarzen Zickzackkurve in der Abbildung 3.11 kann der Verlauf sehr gut nachvollzogen werden. Für den grünen Eigenfrequenzverlauf (sicherer Eigenfrequenzverlauf) wird dagegen gefordert, dass mit zunehmender Stauchung die Sicherheit gegenüber Knicken zunehmen muss. Dies führt zwangsweise zu einer sehr starken Kontaktierung im Anfangsbereich (kleine aufeinanderfolgende Zickzack-Verläufe). Als letztes wird der kritische Eigenfrequenzverlauf eingeführt. Hier darf der Eigenfrequenzverlauf mit zunehmender Stauchung leicht abnehmen. Unter rein theoretischen Gesichtspunkten dürfte dieser Eigenfrequenzverlauf nie zum Knicken führen, weil kein Schnittpunkt mit der Abszisse vorhanden ist. Ob sich dieser Sachverhalt bestätigt, wird im nächsten Abschnitt 3.3.4 diskutiert. Mit Hilfe dieser unterschiedlichen Eigenfrequenzvorgaben werden im Folgenden die zugehörigen Halterungsgeometrien berechnet. In der Abbildung 3.12 sind diese im axialsymmetrischen Schnitt dargestellt. Um ein besseres Verständnis von der gesamten Probekörpergeome-

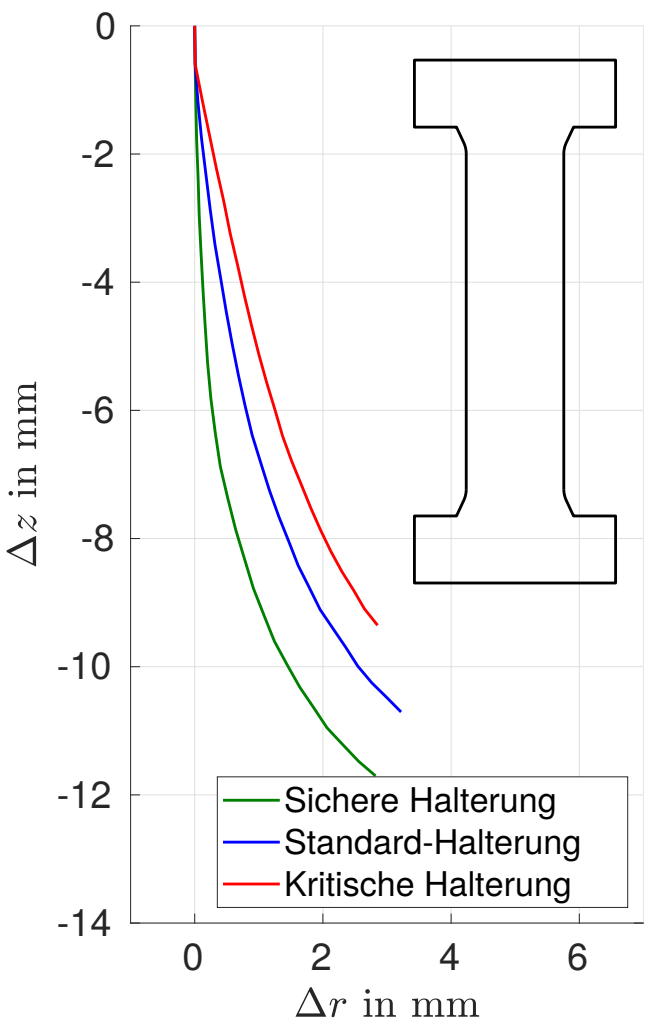

Abb. 3.12 - Parameterstudie für die Halterungskonturen (Simulationsergebnisse)

trie zu bekommen, sei an dieser Stelle der Hantelkopf bereits mit dargestellt. Die spezielle Form des Hantelkopfes dient im Wesentlichen der Realisierung von Zugversuchen. Anhand 
der Abbildung 3.12 wird intuitiv deutlich, dass die sichere Halterung den engsten Verlauf und die kritische Halterung den weitesten Verlauf darstellt. Die Standard-Halterung stellt dabei eine mittlere Lösung dar. Im Weiteren soll der Einfluss der verschiedenen Halterungen im Spannungs-Dehnungsdiagramm untersucht werden. In Abbildung 3.13 werden die Halterungskonturen aus der Parameterstudie mit der homogenen Lösung (Simulation mit einem finiten Element) und der Standard-Hantel (vgl. Alshuth u. a. 2007) verglichen. An-

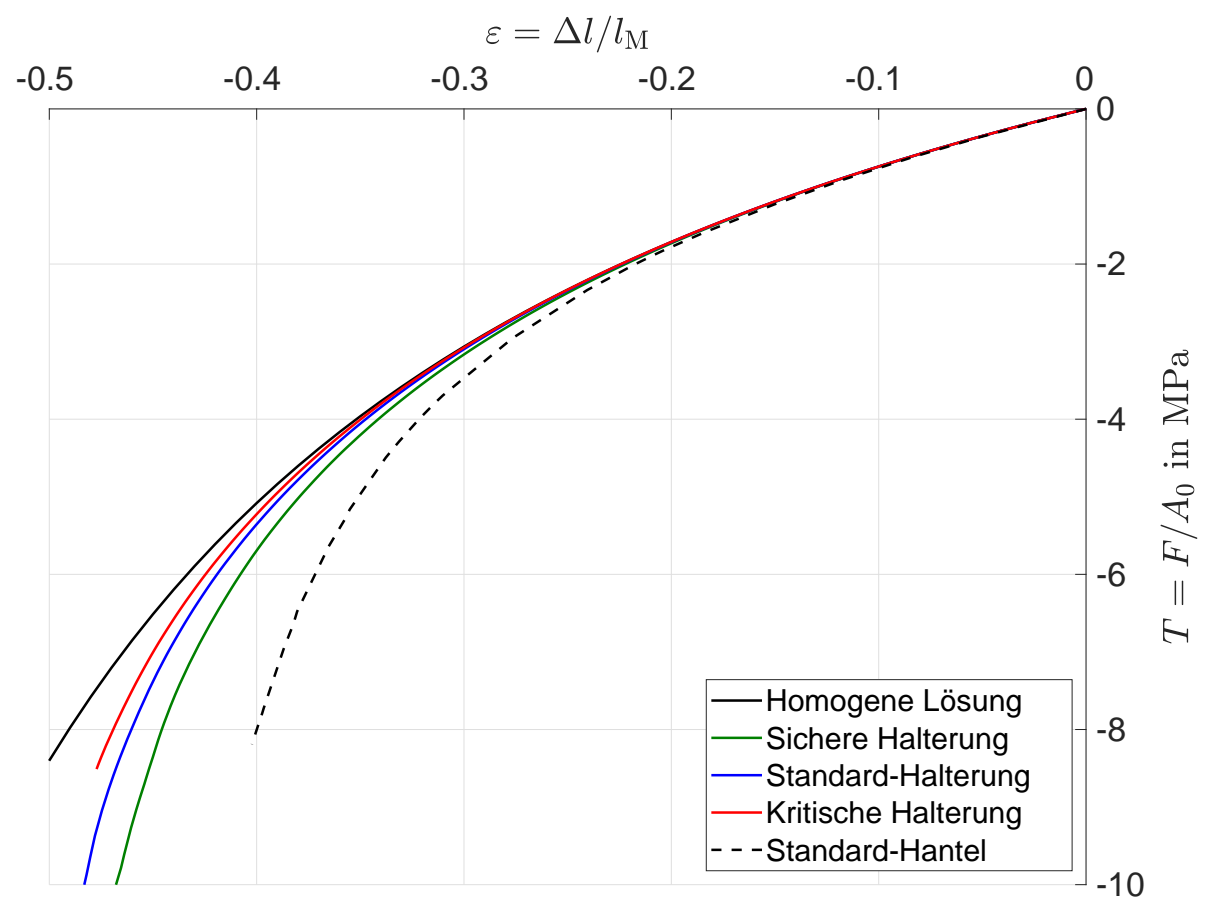

Abb. 3.13 - Spannungs-Dehnungsdiagramm für die verschiedenen Halterungskonturen (Simulationsergebnisse vgl. Tabelle 3.1

hand der Abbildung 3.13 werden eine Reihe interessanter Punkte deutlich. Zunächst kann festgestellt werden, dass die Standard-Hantel die mit Abstand schlechtesten Ergebnisse im Spannungs-Dehnungsdiagramm liefert. Im Gegensatz dazu zeigen die Halterungen, die im Rahmen der Parameterstudie entwickelt worden sind, eine erhebliche Verbesserung. Dies wird zum einen an der verbesserten Homogenität selbst deutlich, zum anderen an der maximal erreichbaren Stauchung.

\subsubsection{Fehler- und Instabilitätsmaße}

Um in direkter Weise Abweichungen zur theoretisch vorliegenden Lösung als auch zum Stabilitätsverhalten der verschiedenen Halterungskonturen angeben zu können, sollen im Folgenden Fehler- und Instabilitätsmaße eingeführt werden. Ziel dabei ist es, die unterschiedlichen Halterungskonturen quantitativ miteinander vergleichen zu können. Zunächst werden die Abweichungen im Spannungs-Dehnungsdiagramm (vergleiche dazu Abbildung 3.13 für die verschiedenen Halterungskonturen zur homogenen Lösung näher betrachtet. Dafür wird das globale Fehlermaß $\eta_{\mathrm{T}}$ eingeführt, welches die relative Differenz zwischen 
der aktuell im Probekörper vorliegenden Spannung $\underset{z}{T} \underset{z}{\mathrm{FE}}(\varepsilon)$ und der theoretisch vorhanden Spannung $\stackrel{\text { th }}{T}_{z z}(\varepsilon)$, dividiert durch die theoretische Spannung beschreibt. In der Gleichung (3-42) ist der Ausdruck dargestellt:

$$
\eta_{\mathrm{T}}:=\left|\frac{\stackrel{\mathrm{FE}}{T}_{z z}(\varepsilon)-\stackrel{\mathrm{th}}{T}_{z z}(\varepsilon)}{\stackrel{\mathrm{th}}{T}_{z z}(\varepsilon)}\right| \quad \operatorname{mit}: \quad \stackrel{\mathrm{FE}}{T}_{z z}(\varepsilon):=\frac{F_{z}(\varepsilon)}{A_{0}} .
$$

Grafisch interpretiert entspricht das globale Fehlermaß dem relativen senkrechten Abstand zwischen homogener Lösung und vorliegender Spannung im Probekörper bei gleicher Stauchung. In der Abbildung 3.14 sind die Fehlermaße $\eta_{\mathrm{T}}$ für die verschiedenen Halterungskonturen dargestellt. Anhand der Abbildung wird deutlich, dass die Standard-Hantel schon

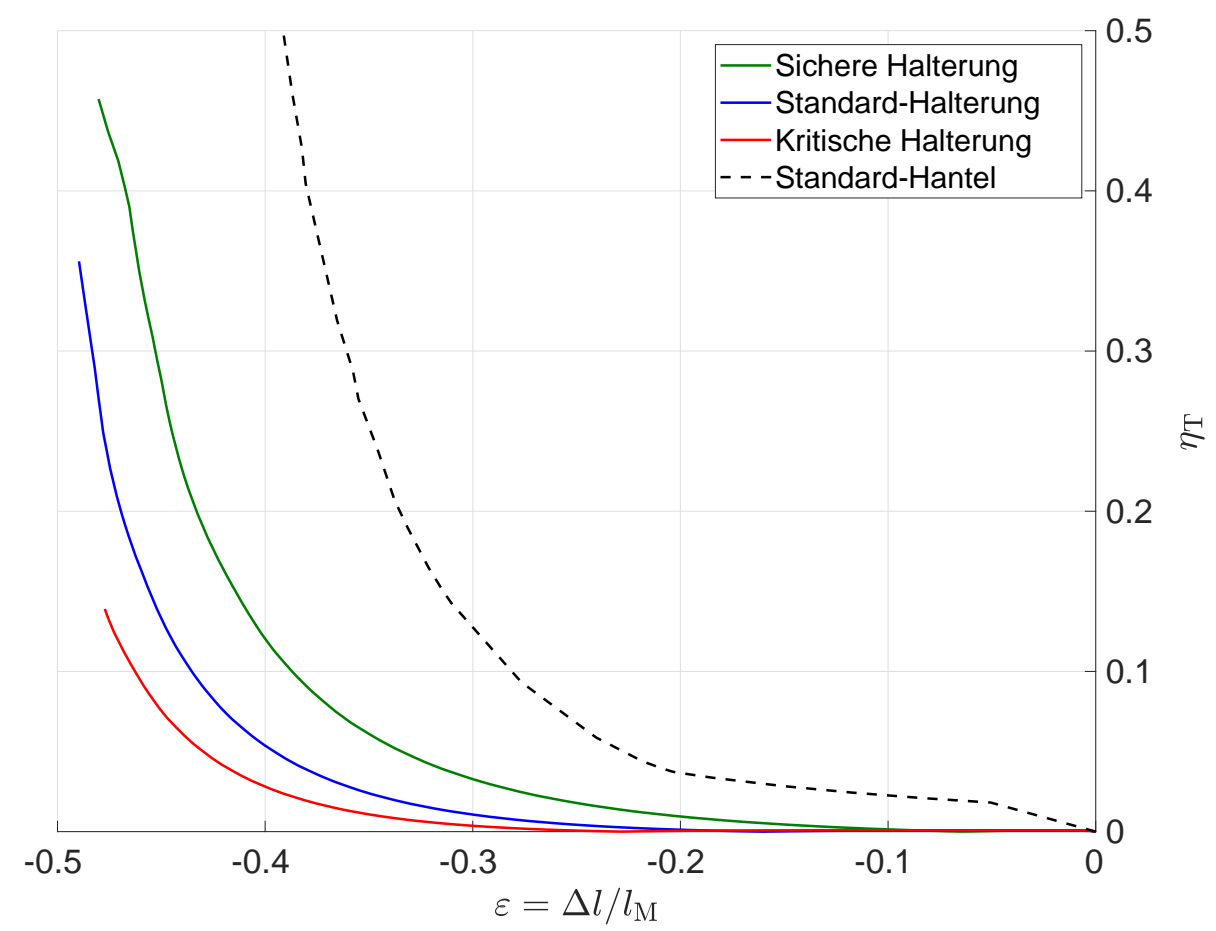

Abb. 3.14 - Darstellung des globalen Fehlermaßes $\eta_{\mathrm{T}}$ für die verschiedenen Halterungskonturen (Simulationsergebnisse vgl. Tabelle 3.1)

bei einer Stauchung von $30 \%$ einen relativen Fehler von über $10 \%$ aufweist. Im Gegensatz dazu besitzen die Halterungen, die im Rahmen der Parameterstudie entwickelt worden sind, signifikant bessere Fehlermaße, selbst bei größeren Stauchungen. Bei einem direkten Vergleich der entwickelten Halterungen untereinander wird deutlich, dass die kritische Halterung das beste Ergebnis erzielt und die sichere Halterung das schlechteste. Dies lässt sich nicht zuletzt auf die gewählten Eigenfrequenzvorgaben zurückführen. Zusammenfassend lässt sich sagen, dass das globale Fehlermaß $\eta_{\mathrm{T}}$ den relativen Messfehler zur homogen Lösung beschreibt und als Maß zur Bewertung des Probekörpers verwendet werden kann. 
Um Aussagen über lokale Abweichungen zur homogenen Druckdeformation treffen zu können, soll im Folgenden ein lokales Fehlermaß verwendet werden. Dieses Maß, das im Rahmen der Arbeit von Naumann (2010) eingeführt worden ist, basiert auf der Grundlage tensorieller Größen und besitzt den Vorteil, dass selbst mehrachsige und inhomogene Spannungszustände mit einer homogenen Druckdeformation verglichen werden können. Es wird dazu das Spannungsmaß $\varsigma$ definiert, das die Differenz zwischen dem lokal vorherrschenden Spannungszustand $\underset{\underline{\sigma}}{\stackrel{\mathrm{FE}}{\underline{E}}}(\varepsilon)$ und dem theoretisch vorhandenen Spannungszustand $\stackrel{\text { th }}{\underline{\underline{T}}}(\varepsilon)$ beschreibt:

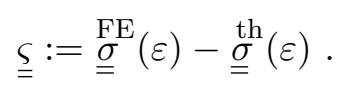

Zum besseren Verständnis werden die Koeffizientenmatrizen mit angegeben:

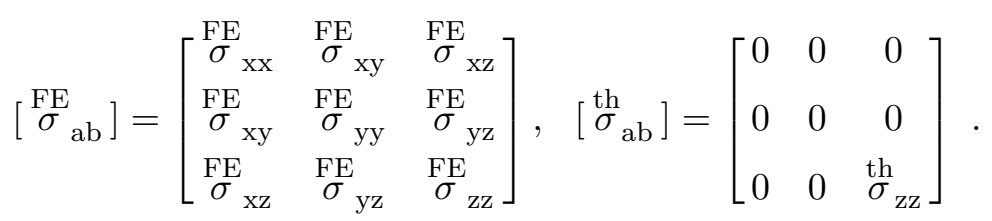

Um ein lokales Vergleichsmaß zu erhalten, soll analog zur Von-Mises-Vergleichsspannung $\sigma_{\text {eqv }}$, die wie folgt definiert ist:

$$
\sigma_{\text {eqv }}=\sqrt{\frac{3}{2}} \sqrt{{\underline{\underline{\sigma^{\prime}}} \cdot \cdot \underline{\underline{\sigma}}^{\prime}}^{\prime}}
$$

ein Vergleichsmaß $\varsigma_{\text {eqv }}$ eingeführt werden:

$$
\varsigma_{\text {eqv }}=\sqrt{\frac{3}{2}} \sqrt{\underline{\underline{\varsigma^{\prime}} \cdot \cdots \underline{\varsigma}^{\prime}}}
$$

Um das lokale Fehlermaß $\eta_{\sigma}$ zu erhalten, wird das vorliegende Vergleichsmaß $\varsigma_{\text {eqv }}$ mit dem theoretisch vorhandenen Vergleichsmaß $\stackrel{\text { th }}{\sigma}_{\text {eqv }}$ normiert. Für den Sonderfall des einachsigen Spannungszustandes $\stackrel{\text { th }}{\sigma_{\text {zz }}}$ kann das lokale Fehlermaß wie folgt vereinfacht werden:

$$
\eta_{\sigma}:=\frac{\varsigma_{\text {eqv }}}{\sigma_{\text {eqv }}}=\frac{\varsigma_{\text {eqv }}}{\sigma_{\mathrm{zz}}}
$$

In der Abbildung 3.15 ist das lokale Fehlermaß $\eta_{\sigma}$ für die verschiedenen Halterungskonturen dargestellt. Aus der Abbildung wird ersichtlich, dass die Standard-Hantel mit der vorliegenden Halterung bei einer Stauchung von 40 \% die größten Abweichungen im Messbereich aufzeigt. Bei einer Dehnungsbestimmung mittels Extensometer führt der inhomogene Randbereich zu großen Fehlern gegenüber der homogenen Druckdeformation. Dies kann zum einen auf die vorliegende Halterung selbst zurückgeführt werden, zum anderen auf den geringen Abstand zwischen Halterung und Extensometer. Vergleicht man die Abbildung 3.15 mit der Abbildung 3.14, dann kann der eben beschriebene Einfluss anhand der schwarz gestrichelten Linie (Standard-Hantel) sehr gut nachvollzogen werden. Im Vergleich dazu zeigen die Halterungskonturen, die im Rahmen der Parameterstudie 


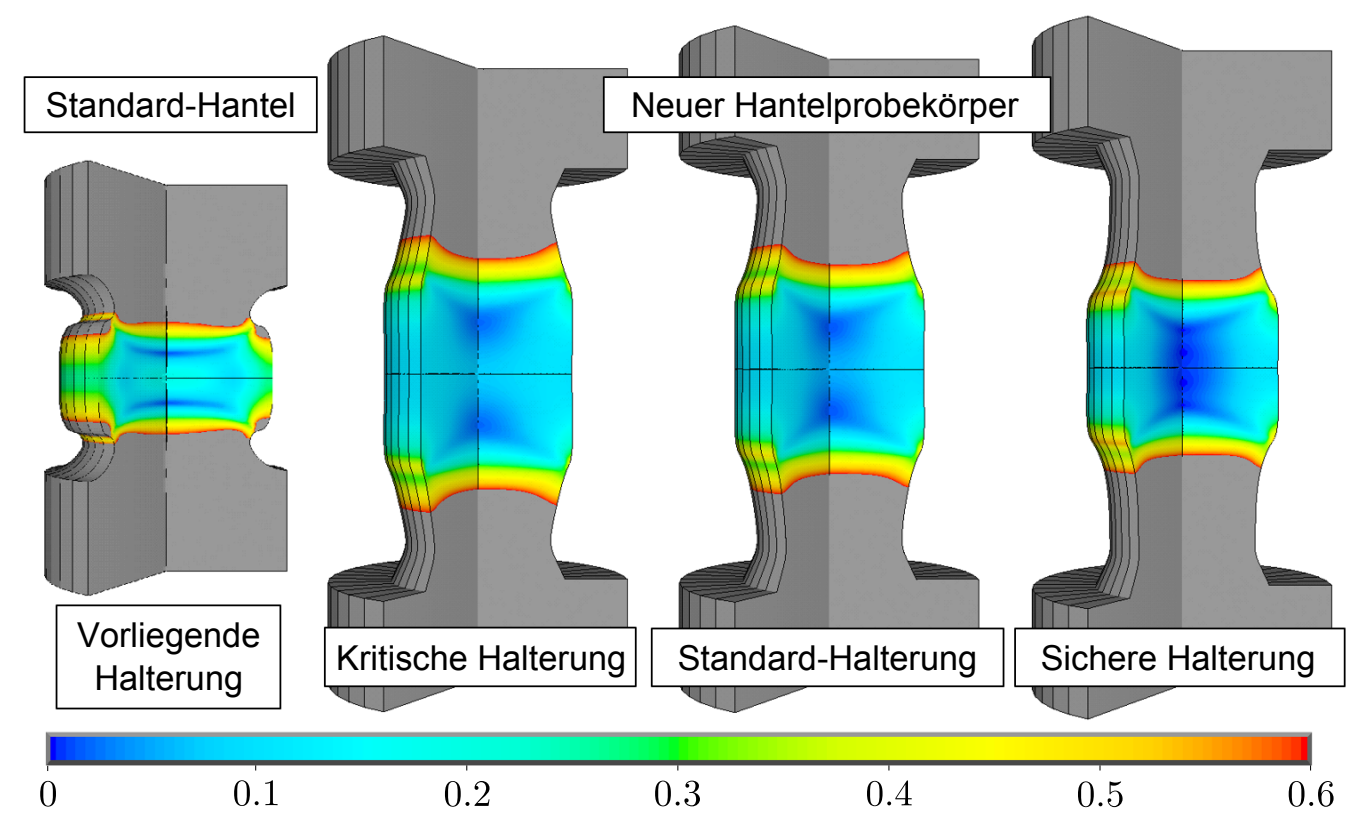

Abb. 3.15 - Darstellung des lokalen Fehlermaßes $\eta_{\sigma}$ für die verschiedenen Halterungskonturen bei einer Stauchung von 40 \% (Abbildung in Anlehnung an die Arbeit von Naumann 2010)

entwickelt worden sind, einen Messbereich mit wesentlich kleineren Abweichungen, siehe Abbildung 3.15. Interessanterweise ist bei allen Hanteln und Halterungskonturen das lokale Fehlermaß $\eta_{\sigma}$ im Innern des Probekörpers verhältnismäßig niedrig. Eine Erklärung dafür bietet der Abstand zu den Halterungskonturen, die in direkter Weise Einfluss auf das Fehlermaß nehmen. Bei genauerer Betrachtung des Innern der Standard-Hantel wird der etwas erhöhte Fehlerbereich deutlich.

Abschließend soll neben den eingeführten Fehlergrößen ein Maß zur Erfassung der Stabilität des Probekörpers definiert werden. Dazu soll zunächst geklärt werden, woher Störungen oder Instabilitäten kommen und wie sie global am besten erfasst werden können. Für reale Probekörper treten unterschiedliche Arten von Störungen bzw. Imperfektionen auf. Dazu zählen neben Geometrieimperfektionen auch Materialinhomogenitäten und der Einfluss unsymmetrischer Randbedingungen. All diese Faktoren wirken sich in unterschiedlicher Art und Weise auf die Homogenität und hier insbesondere auf die Knicksicherheit aus. Demzufolge ist ein Instabilitätsmaß gesucht, welches alle oben beschriebenen Sonderfälle abdeckt und bei beliebigen Stauchungen Auskunft über die Knicksicherheit gibt. Eine sich hierfür eignende Größe stellt die Kinematik der Mittelebene dar (Erste Knickform ist hier von besonderem Interesse). Beispielsweise kann über die Betrachtung der Mittelebene direkt Auskunft gegeben werden, ob der Probekörper sich in einem stabilen oder instabilen Zustand befindet. In der Abbildung 3.16 sind verschiedene Sonderfälle zur Kinematik der Mittelebene (gleichmäßige Aufweitung, Auslenkung und Kombination aus beidem) dargestellt. Anhand der Abbildung 3.16 wird grafisch sehr deutlich, dass eine gleichmäBige Aufweitung der Mittelebene zu keiner Instabilität führen kann. Da es sich später 

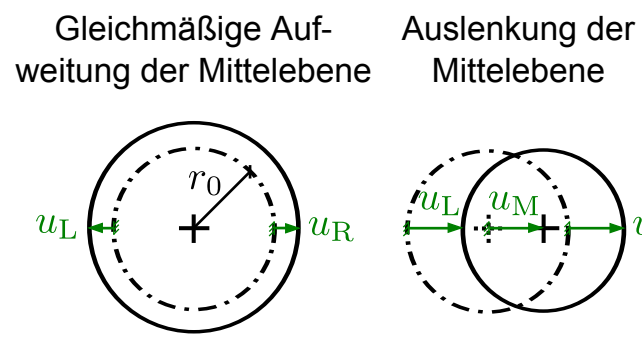

Auslenkung und Aufweitung der Mittelebene

Abb. 3.16 - Darstellung der Verschiebung $u_{\mathrm{L}}$ und $u_{\mathrm{R}}$ für verschiedene Sonderfälle

um kleine Imperfektionen handelt, kann in guter Näherung davon ausgegangen werden, dass der Probekörper seinen Kreisquerschnitt beibehält. Im Gegensatz zur gleichmäßigen Aufweitung ist eine reine Auslenkung der Mittelebene auf das Knicken zurückzuführen (erste Knickform). Das Instabilitätsmaß $\kappa$ wird dabei als Summe der äußeren Verschiebungen $u_{\mathrm{L}}$ und $u_{\mathrm{R}}$, dividiert durch den Ausgangsradius $r_{0}$, definiert. Bei Kenntnis des Mittelpunktversatzes $u_{\mathrm{M}}$ ergibt sich folgender Zusammenhang:

$$
\kappa:=\left|\frac{u_{\mathrm{L}}+u_{\mathrm{R}}}{r_{0}}\right|=\left|\frac{2 u_{\mathrm{M}}}{r_{0}}\right| .
$$

Um die verschiedenen Halterungskonturen miteinander vergleichen und auf ihre Knicksicherheit hin testen zu können, muss eine definierte Anfangsstörung vorgegeben werden. Diese wird in Form einer Geometrieimperfektion erstellt. Im Folgenden wird dies durch eine initiale Auslenkung der Mittelebene um den Betrag $u_{\mathrm{S}}=\iota r_{0}$ realisiert. Dabei beschreibt die Größe $\iota$ den prozentualen Anteil der Auslenkung in Abhängigkeit des Referenzradius. Bei einer nachfolgend aufgebrachten Druckbeanspruchung kann dann das Auswandern der Mittelebene beobachtet werden. Weiterhin wurde neben der Geometrieimperfektion von $\iota=0.01$ ein Schrumpf von $1 \%$ der Probe gegenüber der Halterung realisiert. Diese Größen sollen reale Störungen durch den Herstellungs- oder Vulkanisationsprozess abbilden. In Abbildung 3.17 sind die Instabilitätsmaße für die verschiedenen Halterungskonturen dargestellt. Anhand der Abbildung 3.17 werden folgende Sachverhalte deutlich: Die sichere Halterung besitzt das mit Abstand niedrigste Instabilitätsmaß, weist also die größte Sicherheit gegenüber Geometrieimperfektionen und Schrumpf auf. Im Gegensatz dazu besitzt die kritische Halterung das größte Instabilitätsmaß, verhält sich also sehr sensitiv gegenüber Imperfektionen. Vergleicht man die Farbverläufe mit der Abbildung 3.14, so ist ein gegenläufiger Trend festzustellen. Hier besitzt die kritische Halterung das niedrigste und die sichere Halterung das größte Fehlermaß. Die Standard-Halterung stellt in beiden Abbildungen eine mittlere Lösung dar. Zusammenfassend lässt sich sagen, dass mit Hilfe der entwickelten Maße qualitative Aussagen über die Abweichung zu einer theoretisch vorhandenen Lösung (Simulation mit einem finiten Element) und zur Stabilität der verschiedenen Halterungskonturen getroffen werden können. Die in der Parameterstudie designten Halterungskonturen weisen diesbezüglich unterschiedliche Vor- und Nachteile 


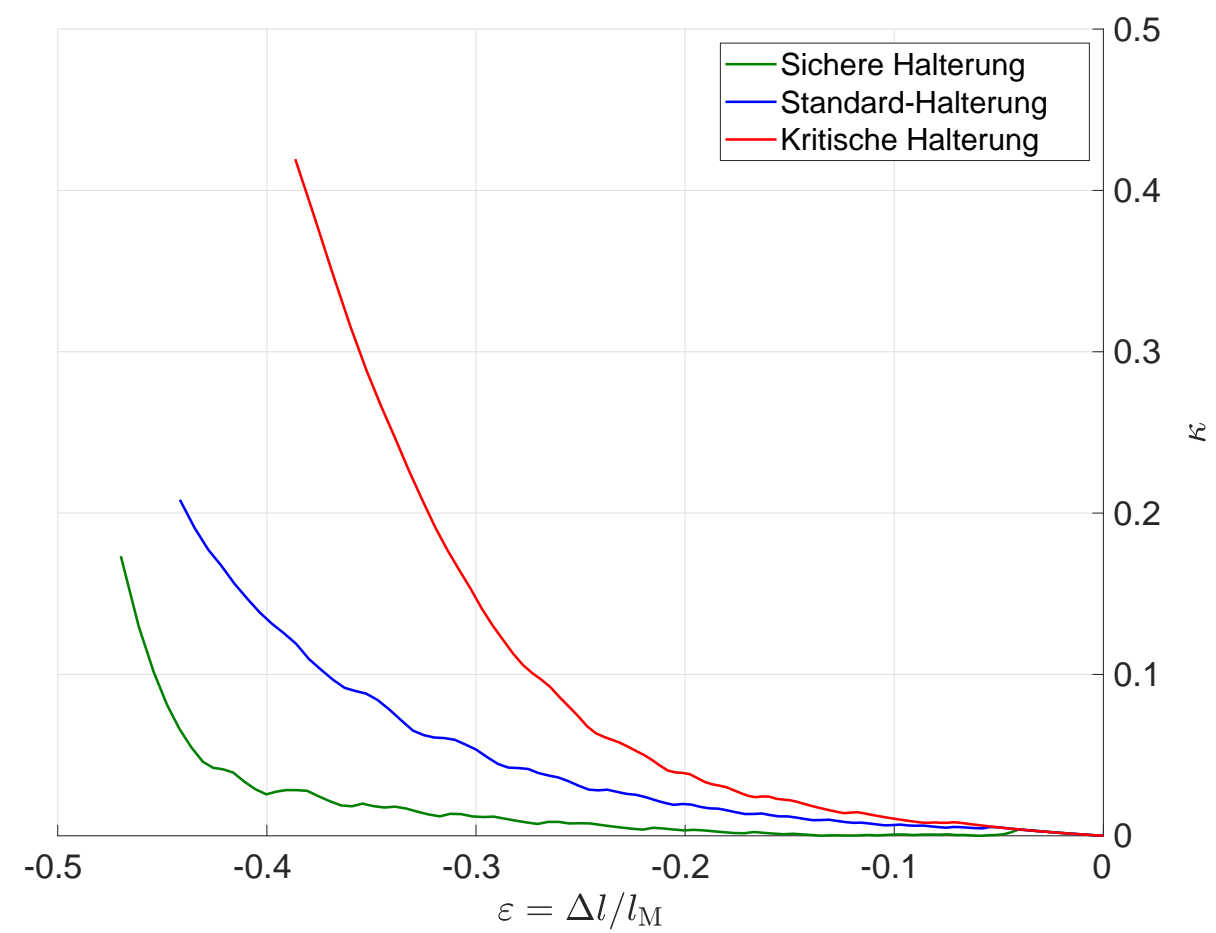

Abb. 3.17 - Darstellung des Instabilitätsmaßes $\kappa$ für die verschiedenen Halterungskonturen (Simulationsergebnisse vgl. Tabelle 3.1

auf. Ziel der nachfolgenden Abschnitte ist zum einen die Optimierung der Probekörperlänge, zum anderen die Formoptimierung der Halterungskontur mittels der eingeführten Fehler- und Instabilitätsmaße.

\subsubsection{Optimierung der Probekörperlänge}

In diesem Abschnitt soll die Optimierung der Probekörperlänge $l_{0}$ bei Verwendung des entwickelten Halterungsalgorithmus erfolgen. Die Optimierung soll in der Weise durchgeführt werden, dass die Probekörperlänge ideale Eigenschaften hinsichtlich auftretender Fehlergrößen als auch Stabilität besitzt. Die Zielfunktion wird dabei wie folgt formuliert:

$$
f:=\alpha \int_{0}^{\bar{\varepsilon}} \eta_{\mathrm{T}}(\varepsilon) \mathrm{d} \varepsilon+(1-\alpha) \int_{0}^{\bar{\varepsilon}} \kappa(\varepsilon) \mathrm{d} \varepsilon .
$$

Der erste Term beschreibt dabei den globalen Messfehler $\eta_{\mathrm{T}}$, welcher die relative Abweichung zur theoretisch vorhandenen Lösung angibt. Der zweite Term beschreibt das Maß der Instabilität $\kappa$, welches die Gefahr gegenüber Knicken angibt. Somit sind beide Terme in der Zielfunktion als globale Größen definiert, welche einen direkten Einfluss auf die Probekörperlänge selbst ausüben. Über den Faktor $\alpha \in[0,1]$ kann eine entsprechende Gewichtung der beiden Zielfunktionswerte vorgenommen werden. Die Zielfunktion wird innerhalb einer Matlab-Umgebung implementiert und mit Hilfe der Optimierungstoolbox (Simplex-Verfahren) gelöst. In Abbildung 3.18 ist der schematische Ablaufplan zur Identi- 
fizierung der idealen Probekörperlänge dargestellt. Neben dem Startwert $l_{0}$ muss weiterhin

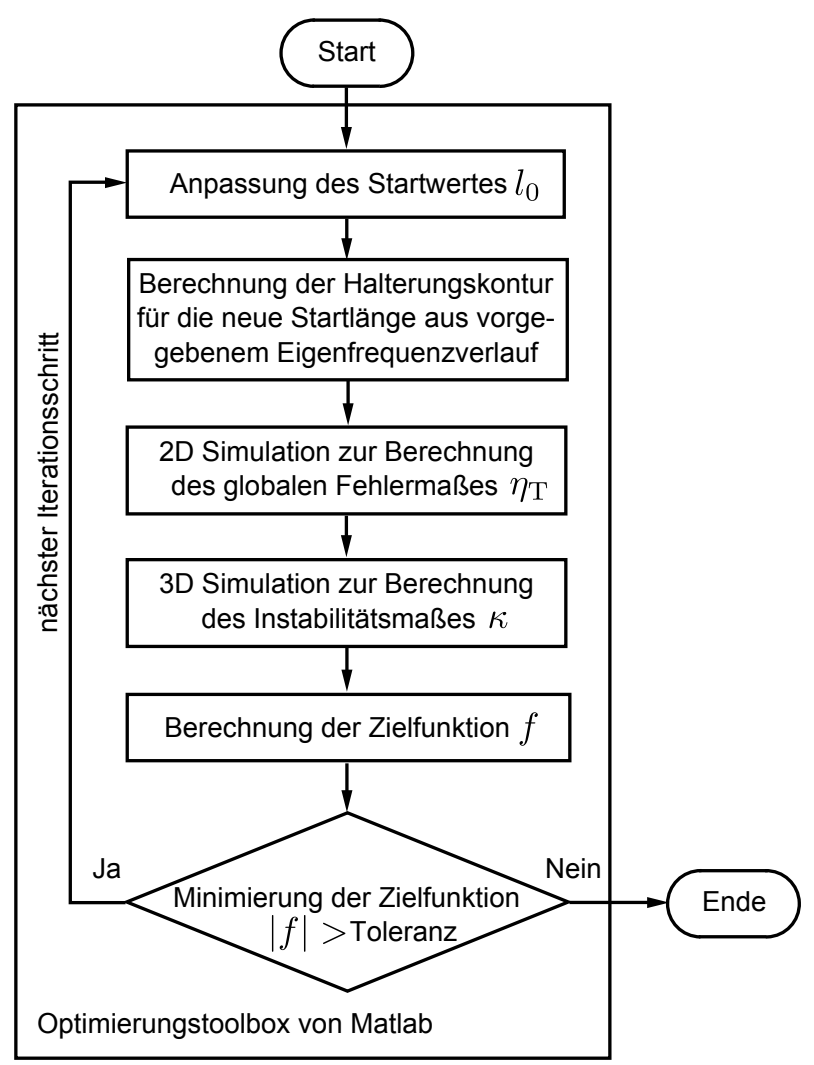

Abb. 3.18 - Ablaufplan für die Optimierung der Probekörperlänge

ein vorgegebener Eigenfrequenzverlauf für die Halterungsberechnung angegeben werden. Da dieser gewählte Eigenfrequenzverlauf einen signifikanten Einfluss auf die nachfolgende Optimierung hat, soll im nächsten Unterabschnitt gesondert darauf eingegangen werden. Nach der Berechnung der Halterungskontur erfolgen die Simulationen zur Bestimmung des Fehlermaßes $\eta_{\mathrm{T}}$ und des Instabilitätsmaßes $\kappa$. Die beiden Funktionswerte werden entsprechend ihrer Grenzen aufintegriert und im Anschluss mit der gewählten Gewichtung zum Zielfunktionswert verrechnet. Mit Hilfe des Simplex-Verfahrens (vgl. Bronstein u. a. 2012) kann dann der nächste Iterationsschritt durchgeführt werden. Die Optimierung endet, wenn der Zielfunktionswert $f$ kleiner als die vorgegebene Toleranz ist.

\section{Untersuchung der Eigenfrequenzvorgaben für die Optimierung}

Da die vorgegebenen Eigenfrequenzverläufe einen entscheidenden Einfluss auf die Halterungsberechnung und die damit verbundene Optimierung haben, soll im Folgenden gesondert darauf eingegangen werden. Hierfür werden zunächst zwei verschiedene Designvarianten vorgestellt und im Anschluss miteinander verglichen. Die erste Variante, die hier vorgeschlagen werden soll und für eine Optimierung herangezogenen werden kann, ist die Verwendung von konstanten Eigenfrequenzverläufen $\omega^{*} / \omega_{0}=1$. Diese Variante zeichnet sich unter anderem dadurch aus, dass die Eigenfrequenz, die zu Beginn der Modalanalyse 
berechnet worden ist, über den kompletten Stauchprozess konstant gehalten wird. In der

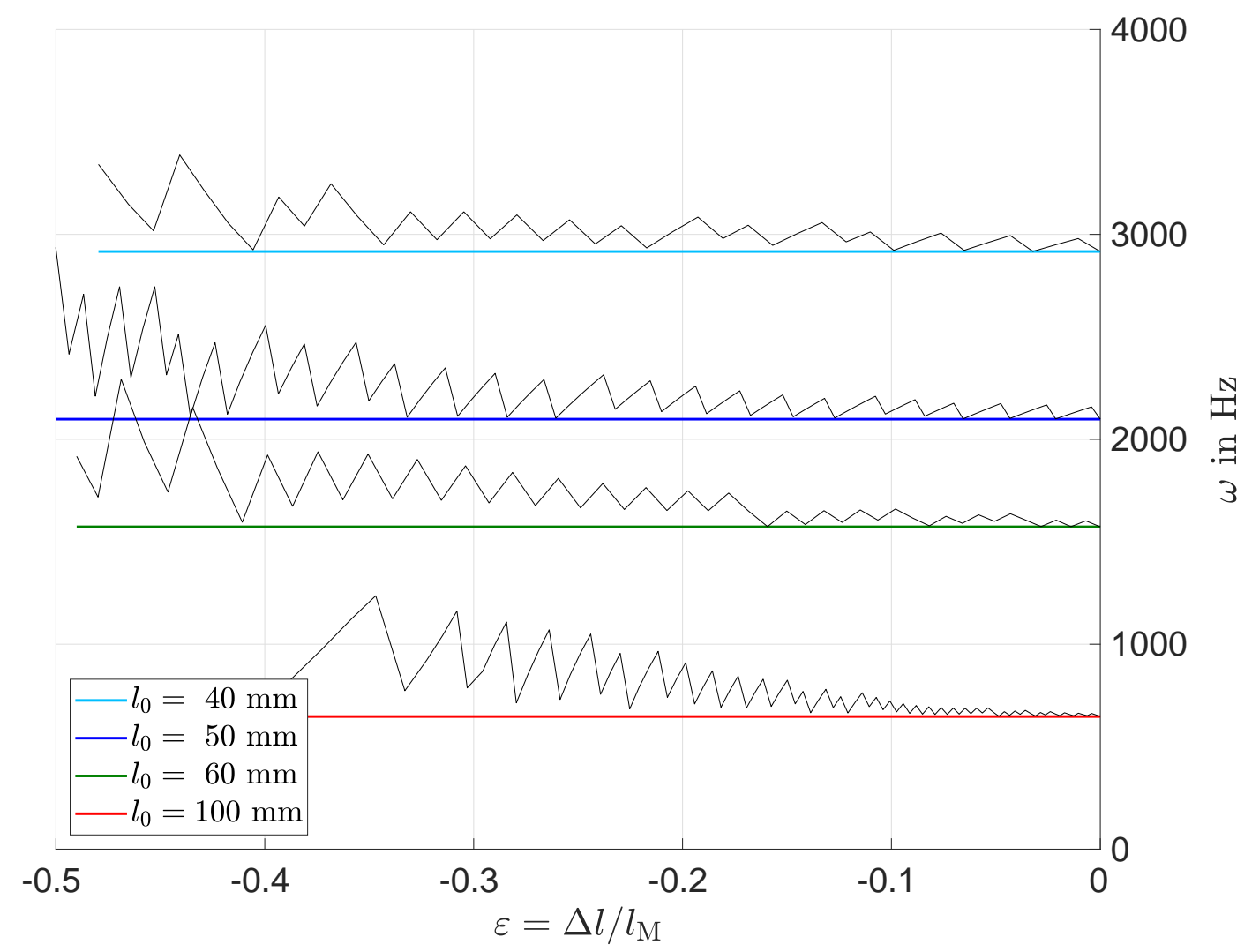

Abb. 3.19 - Darstellung der konstanten Eigenfrequenzvorgaben für unterschiedliche Probekörperlängen $l_{0} \in[40,100] \mathrm{mm}$ sowie der aus dem Halterungsalgorithmus berechneten Eigenfrequenzverläufe (Simulationsergebnisse vgl. Tabelle 3.1

Abbildung 3.19 ist dies zum besseren Verständnis dargestellt. Es ist hierbei zu beachten, dass die Eigenfrequenz abhängig von der jeweiligen Ausgangslänge konstant gehalten wird. Dies führt zwangsweise zu Halterungskonturen, die schon zu Beginn der Berechnung ganz unterschiedliche Reserven bezüglich auftretender Fehlergrößen als auch Stabilität besitzen. In nachfolgenden Untersuchungen soll geklärt werden, ob die Halterungskonturen, die aus diesen Eigenfrequenzverläufen berechnet werden, in direkter Weise miteinander verglichen werden dürfen. Beispielsweise besitzt eine Probekörperlänge $l_{0}=40 \mathrm{~mm}$ schon zu Beginn der Berechnung eine ganz andere Knicksicherheit als eine Probekörperlänge mit $l_{0}=100 \mathrm{~mm}$. Umgekehrt verhält es sich mit dem relativen Messfehler $\eta_{\mathrm{T}}$.

Aus diesem Grund soll für die zweite Designvariante ein Verlauf vorgeschlagen werden, der auf ein identisches Eigenfrequenzniveau führt. Dies soll im Weiteren mit identischen asymptotischen Eigenfrequenzverläufen bezeichnet werden. In der Abbildung 3.20 ist dies für die oben gezeigten Ausgangslängen dargestellt. Die Anpassung auf ein identisches Eigenfrequenzniveau (hier: Mittelteillänge $l_{0}=50 \mathrm{~mm}$ ) erfolgte in der Weise, dass bei einer Stauchung von $30 \%$ die Verläufe ineinander übergehen. Für den Übergangsbereich 


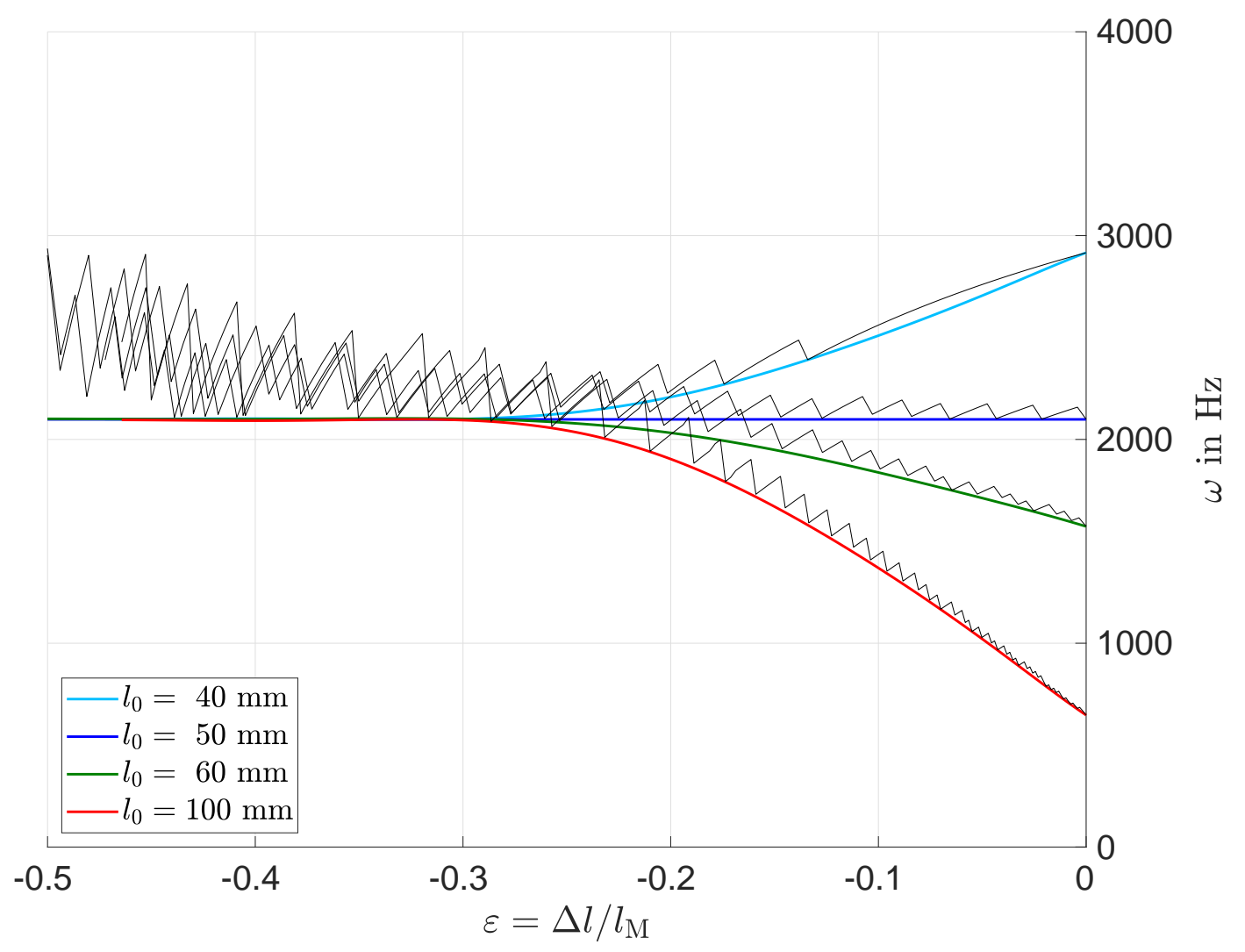

Abb. 3.20 - Darstellung der identischen asymptotischen Eigenfrequenzvorgaben für unterschiedliche Probekörperlängen $l_{0} \in[40,100] \mathrm{mm}$ sowie der aus dem Halterungsalgorithmus berechneten Eigenfrequenzverläufe (Simulationsergebnisse vgl. Tabelle 3.1

$\varepsilon \in[-0.3,0]$ werden Sinusfunktionen verwendet, die tangential am Umkehrpunkt abschließen. Der abgebildete Eigenfrequenzverlauf der Mittelteillänge $l_{0}=100 \mathrm{~mm}$ dient als Grenzfall des Algorithmus zur Halterungsberechnung. Hieran kann gezeigt werden, dass eine weitere Erhöhung der Mittelteillänge nur dazu führt, dass die Halterungskontur sich einem beliebig langen rohrförmigen Abschnitt anschmiegt. In Abbildung 3.21 sind die verschiedenen Halterungskonturen, die aus den oben gezeigten Eigenfrequenzvorgaben berechnet worden sind, dargestellt. Um eine bessere Vergleichbarkeit zu ermöglichen, sind die Konturen um die jeweils fehlende Mittelteillänge verschoben worden. Der nahezu horizontale Verlauf der Halterungskontur für $l_{0}=100 \mathrm{~mm}$ lässt darauf schließen, dass eine kontinuierliche Fixierung von Knoten in der Halterungsberechnung stattgefunden hat. Vergleicht man dazu die entsprechende Eigenfrequenzvorgabe in der Abbildung 3.20, dann kann dies anhand der sehr kleinen Zickzack-Verläufe sehr gut nachvollzogen werden (siehe $l_{0}=100 \mathrm{~mm}$ im Anfangsbereich). Aus der Abbildung 3.21 ist weiterhin gut zu erkennen, dass sich alle Halterungskonturen in ihrem prinzipiellen Verlauf stark ähneln. Alle hier dargestellten Konturen schmiegen sich, beginnend von ihrem Startpunkt, sehr gut asymptotisch aneinander an. Dies ist nicht zuletzt auf die geforderte Eigenfrequenzvorgabe zurückzuführen. 


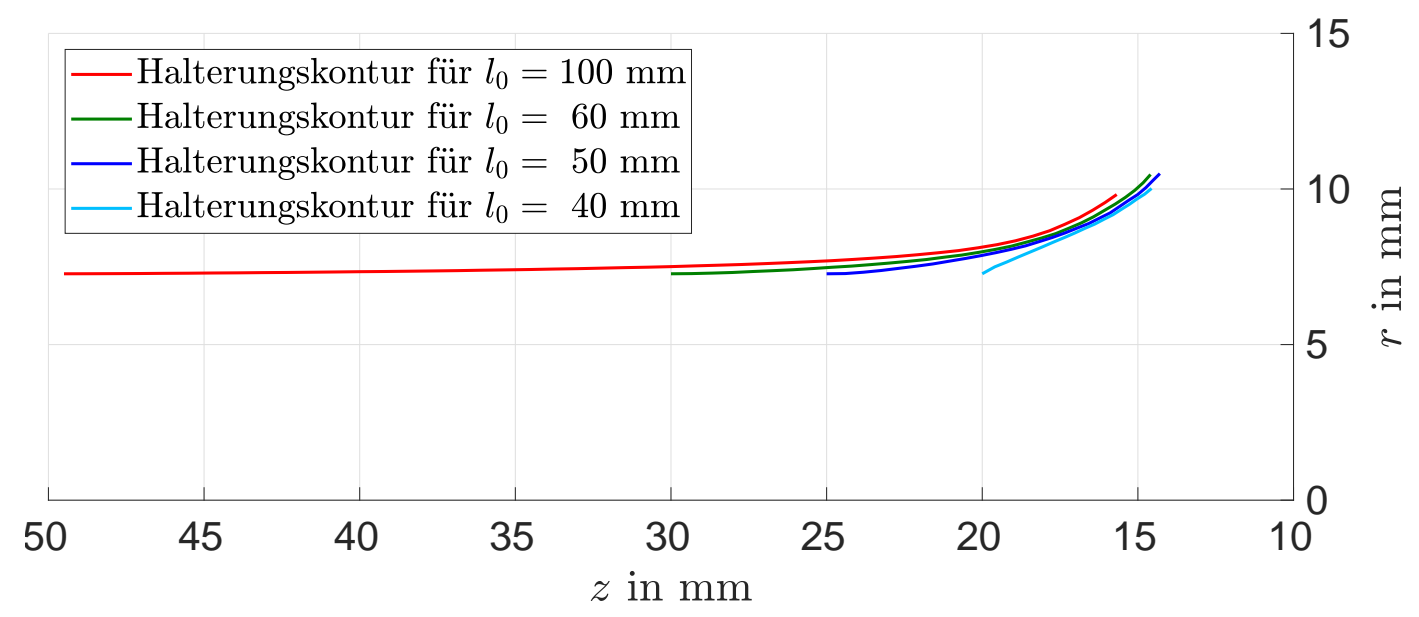

Abb. 3.21 - Gegenüberstellung der berechneten Halterungskonturen die aus den vorgegebenen Eigenfrequenzverläufen ermittelt worden sind (Simulationsergebnisse)

Um abschließende Aussagen über die Eignung der Eigenfrequenzvorgaben treffen zu können, sollen im Weiteren beide Varianten für die Optimierung herangezogen werden. Im Folgenden wird dazu der Verlauf der Zielfunktionslandschaft näher betrachtet.

\section{Darstellung der Zielfunktionslandschaft}

Auf der Grundlage der verschiedenen Eigenfrequenzvorgaben soll nun die Zielfunktionslandschaft näher untersucht werden. Das prinzipielle Vorgehen kann dabei der Abbildung 3.18 entnommen werden. Da die zu optimierende Größe (Probekörperlänge) nicht unabhängig vom Radius bestimmt werden kann, soll dieser zunächst festgelegt werden. Ausgehend von der Standard-Hantel (vgl. Alshuth u. a.) bietet es sich an den Radius, auch aus herstellungsbedingten Gründen, mit $r_{0}=7.5 \mathrm{~mm}$ festzulegen (unter Berücksichtigung von $3 \%$ Schrumpf ergibt sich ein Radius $r=7.275 \mathrm{~mm}$ ). Ein sinnvoller Bereich zur Darstellung der Zielfunktionslandschaft, in Abhängigkeit des zuvor festgelegten Radius, bewegt sich in den Grenzen von $l_{0} \in[35,60] \mathrm{mm}$. Eine Mittelteillänge die kleiner als $35 \mathrm{~mm}$ ist führt nur zu großen Abweichungen im Messbereich (vgl. Abbildung 3.14). Eine Mittelteillänge größer $60 \mathrm{~mm}$ macht aus stabilitätstechnischen Gründen wenig Sinn, da sich die daraus ergebende Halterungskontur nur noch einem rohrförmigen Abschnitt anfügt (vgl. Abbildung 3.21). Die Zielfunktionslandschaft wird in $0.5 \mathrm{~mm}$ Schritten diskretisiert. Eine feinere Auflösung der Probekörperlänge ist aus herstellungsbedingten Gründen nicht praktikabel. Es sei an dieser Stelle darauf hingewiesen, dass eine Skalierung der Zylindergeometrie bezüglich des Längen-Durchmesser-Verhältnisses für andere Anwendungsfälle mit den hier dargestellten Methoden ebenso denkbar wäre.

Für ein besseres Verständnis der Zielfunktionslandschaft werden zunächst die einzelnen Bestandteile der Zielfunktion (siehe Gleichung (3-49) dargestellt. In der Abbildung 3.22 
und der Abbildung 3.24 ist dies jeweils für konstante und identische asymptotische Eigenfrequenzvorgaben zu sehen. Zunächst soll die Abbildung 3.22 näher betrachtet werden.

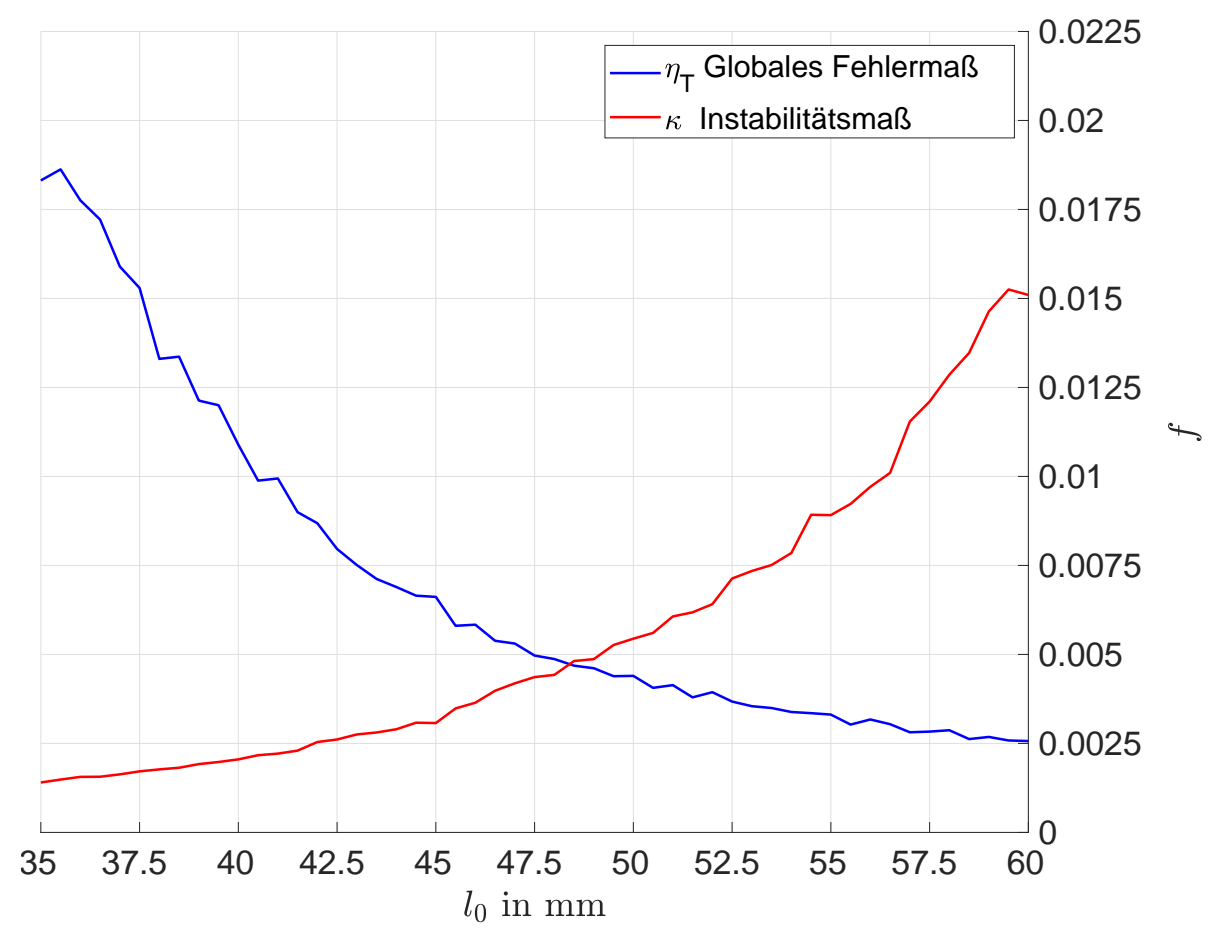

Abb. 3.22 - Darstellung der Zielfunktionsanteile $\eta_{\mathrm{T}}$ und $\kappa$ für konstante Eigenfrequenzvorgaben (Simulationsergebnisse vgl. Tabelle 3.1. Gewichtung $\alpha=0.6)$

Die konstanten Eigenfrequenzvorgaben führen, wie bereits vermutet, zu gegensätzlichen Verläufen der Zielfunktionswerte. Es wird hier ersichtlich, dass mit zunehmender Probekörperlänge das globale Fehlermaß abnimmt und das Maß der Instabilität anwächst. Beide Verläufe lassen sich dadurch erklären, dass die Eigenfrequenz, die zu Beginn der Modalanalyse berechnet worden ist, über den kompletten Stauchprozess konstant gehalten wird. Die verschiedenen Probekörperlängen starten so mit unterschiedlichen Reserven bezüglich auftretender Fehlergrößen und auch Stabilität. In Abbildung 3.23 ist der komplette Zielfunktionsverlauf für eine Gewichtung von $\alpha=0.6$ dargestellt. Um Fluktuation zu vermeiden, wurden die $\alpha$-Werte in 0.1 Schritten variiert. Dabei kam heraus, dass bei $\alpha=0.6$ ähnlich große Zielfunktionsanteile vorhanden sind. Aus dem Zielfunktionsverlauf $f$ kann die optimale Probekörperlänge bei $l_{0}=45.5 \mathrm{~mm}$ abgelesen werden. Es sei an dieser Stelle darauf hingewiesen, dass sich das Minimum der Optimierung in einer nahezu horizontalen Senke für $l_{0} \in[45,48.5] \mathrm{mm}$ befindet. Dieses Ergebnis ist insofern interessant, als dass eine leichte Abweichung des Zielfunktionswertes nicht gleich zu signifikant schlechteren Resultaten führt. Im Folgenden soll nun die Abbildung 3.24 näher betrachtet werden. Zunächst wird deutlich, dass identische asymptotische Eigenfrequenzvorgaben zu einem nahezu horizontalen Verlauf der Zielfunktionswerte führen. Erst bei einer Probekörperlänge kleiner $40 \mathrm{~mm}$ wird das globale Fehlermaß zunehmend größer. Für die hier gewählte Eigenfrequenzvorgabe bedeutet dies, dass die Halterung den Anforde- 


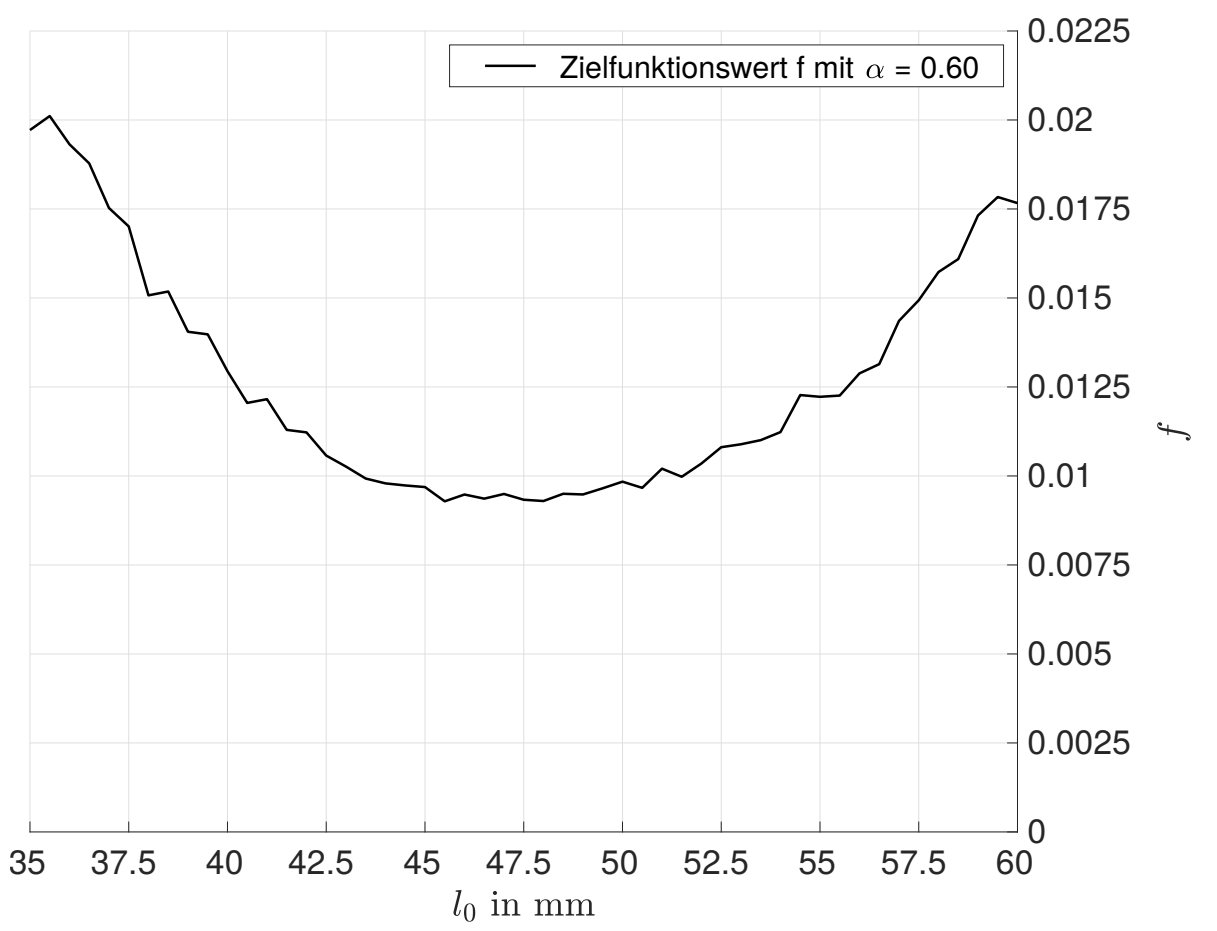

Abb. 3.23 - Darstellung der Zielfunktion $f$ für konstante Eigenfrequenzvorgaben (Simulationsergebnis vgl. Tabelle 3.1

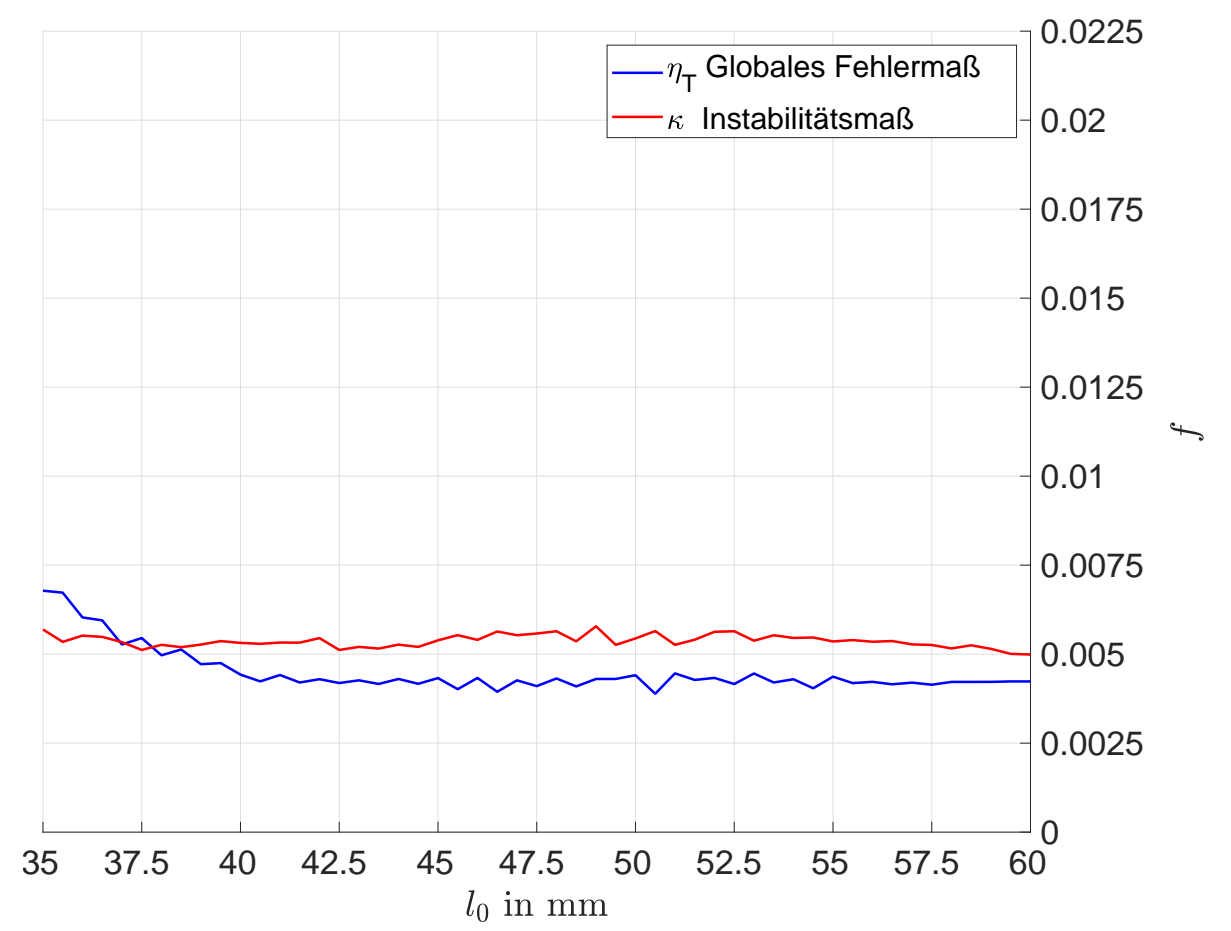

Abb. 3.24 - Darstellung der Zielfunktionsanteile $\eta_{\mathrm{T}}$ und $\kappa$ für identische asymptotische Eigenfrequenzvorgaben (Simulationsergebnisse vgl. Tabelle 3.1 . Gewichtung $\alpha=0.6$ )

rungen bezüglich auftretender Messfehler nicht mehr nachkommen kann und somit das globale Fehlermaß ansteigt. In der Abbildung 3.25 ist der gesamte Zielfunktionsverlauf für 


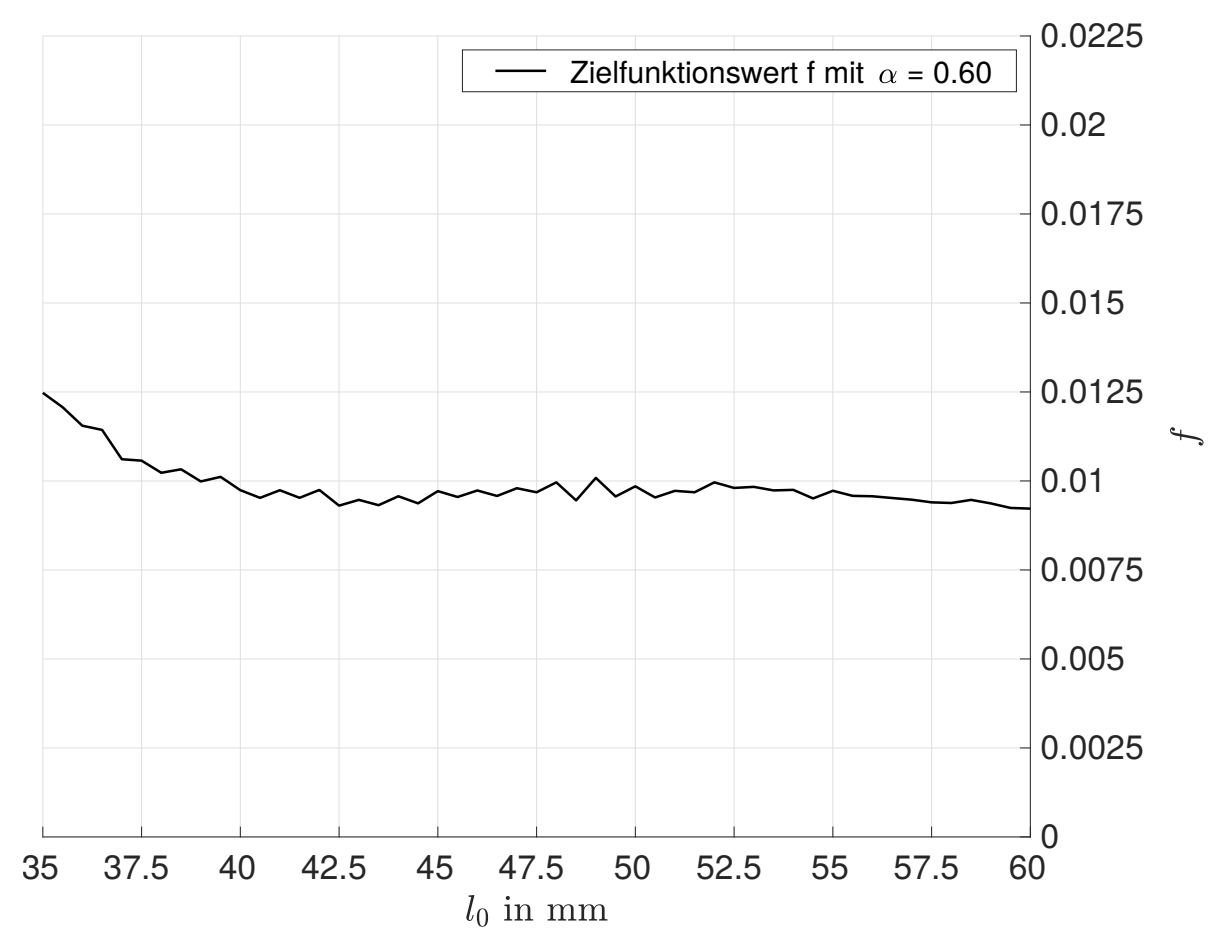

Abb. 3.25 - Darstellung der Zielfunktion $f$ für identische asymptotische Eigenfrequenzvorgaben (Simulationsergebnis vgl. Tabelle 3.1 .

eine Gewichtung von $\alpha=0.6$ dargestellt. Die Gewichtung erfolgte wieder in der Weise, dass zum einen die einzelnen Zielfunktionswerte die gleiche Größenordnung besitzen (vgl. dazu Abbildung 3.22 und Abbildung 3.24 und zum anderen die Zielfunktionslandschaften auch untereinander direkt miteinander verglichen werden können. Aus dem Zielfunktionsverlauf wird deutlich, dass unterschiedliche Probekörperlängen bei der hier verwendeten Eigenfrequenzstrategie nahezu identisch aufeinander abgestimmt werden können. Soll der Probekörper auch unter den Gesichtspunkten der Materialersparnis optimiert werden, so kristallisiert sich ein Maß von $l_{0}=40 \mathrm{~mm}$ heraus. Größere Probekörperlängen besitzen bei der hier gewählten Eigenfrequenzvorgabe keinen merklichen Vorteil. In der Abbildung 3.26 sollen dazu abschließend einige ausgewählte Probekörperlängen mit zugehörigen Halterungen dargestellt und verglichen werden. Um eine bessere Vergleichbarkeit zu ermöglichen, wird hier das lokale Fehlermaß $\eta_{\sigma}$ dargestellt. Dieses Maß gibt die lokale Abweichung zwischen einer homogenen Stauchung und der aktuellen Stauchung des Probekörper an, vgl. Gleichung (3-47). Die Betrachtung der einzelnen Probekörperlängen bestätigt das Ergebnis der Zielfunktionslandschaft in Abbildung 3.25. Die Mittelteillänge $l_{0}=100 \mathrm{~mm}$ mit entsprechender Halterungskontur dient hier als Grenzfall der Untersuchung. Deutlich wird, dass für die nächsten Mittelteillängen keine signifikanten Unterschiede zu verzeichnen sind. Erst bei einer weiteren Reduzierung der Mittelteillänge $l_{0} \leq 40 \mathrm{~mm}$ sind im Randbereich, aufgrund des verkleinerten Messbereichs, größer werdende Abweichungen zu erkennen. Dieser Fehlerbereich wächst bei noch größeren Stauchungen weiter an, weswegen die Probekörperlänge $l_{0}=40 \mathrm{~mm}$ als unterster Grenzfall der verwendeten Eigenfrequenz- 


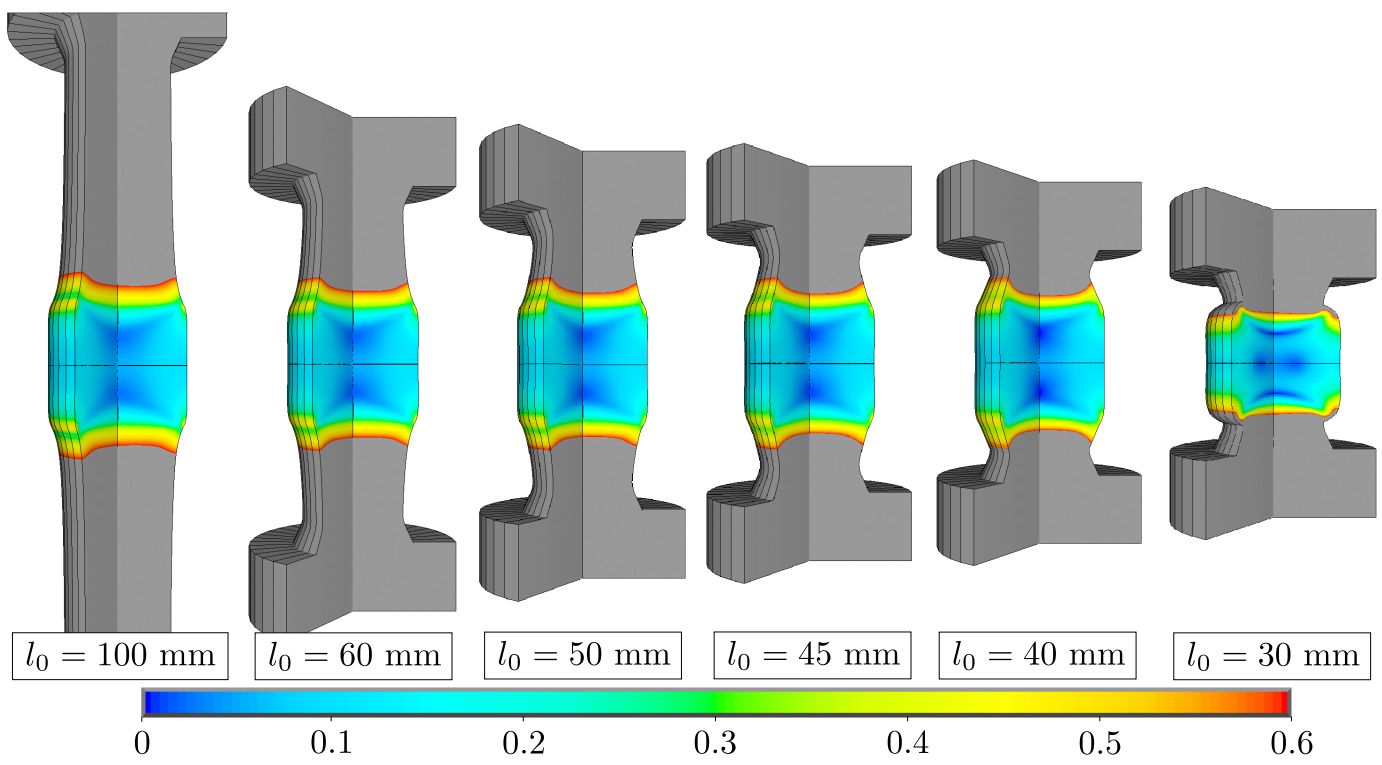

Abb. 3.26 - Darstellung des lokalen Fehlermaßes $\eta_{\sigma}$ für verschiedene Probekörperlängen $l_{0} \in[100,30] \mathrm{mm}$ bei einer Stauchung von $40 \%$

strategie angesehen werden sollte. Hinzu kommt, dass bei Mittelteillängen $l_{0} \leq 40 \mathrm{~mm}$ ab größeren Stauchungen das virtuelle Extensometer von der Halterungskontur verdeckt wird.

\section{Zusammenfassung der Optimierung der Probekörperlänge:}

Aus den Analysen der verschiedenen Zielfunktionslandschaften lassen sich eine Reihe interessanter Schlussfolgerungen ziehen. Bei der Modellierung mit identischen asymptotischen Eigenfrequenzvorgaben kann festgestellt werden, dass unterschiedliche Mittelteillängen nahezu beliebig aufeinander abgestimmt werden können. Erst bei einer Mittelteillänge kleiner $l_{0}=40 \mathrm{~mm}$ treten merkliche Unterschiede im Messbereich auf. Unter dem Gesichtspunkt der Materialersparnis sollte die kleinstmögliche Mittelteillänge bei Beibehaltung des geringen Messfehlers bevorzugt werden. In weiteren Analysen wurde untersucht, inwieweit die Zielfunktionslandschaft ihre Charakteristik beibehält, wenn das identische Eigenfrequenzniveau als auch der Schnittpunkt des Übergangs variiert wird. Dazu wurde das identische Eigenfrequenzniveau für Ausgangslängen von $l_{0}=45 \mathrm{~mm}$ bzw. $l_{0}=55 \mathrm{~mm}$ sowie der Schnittpunkt, an dem die Eigenfrequenzverläufe ineinander übergehen, angepasst (hier bei $20 \%$ bzw. 40\% Stauchung). Alle Variationen führten zu ähnlichen Verläufen der Zielfunktionslandschaft, womit die Charakteristik der Funktion in Abbildung 3.25 bestätigt wird. Im Anhang sind dazu einige ausgewählte Beispiele dargestellt, siehe Abbildung A.1 und Abbildung A.2. Aus den Analysen der Zielfunktionslandschaft mit konstanten Eigenfrequenzvorgaben ergab sich ein Optimum bei $l_{0}=45.5 \mathrm{~mm}$. Es muss an dieser Stelle darauf hingewiesen werden, dass die Verwendung von konstanten Eigenfrequenzvorgaben zu einem ungünstig konditionierten Zielfunktionsverlauf führt und besonderes an den Grenzen schlechte Zielfunktionswerte liefert. Für weitere Untersuchungen sollte auf diese Eigenfrequenzvorgabe verzichtet werden. 
Die ideale Probekörperlänge wird anhand der oben genannten Gesichtspunkte bei $l_{0}=$ $45.5 \mathrm{~mm}$ festgelegt. Diese Länge stellt bei Betrachtung der Abbildung 3.23 als auch der Abbildung 3.25 und unter dem Aspekt der Materialersparnis das beste Ergebnis der Optimierung dar. Es ist dabei zu beachten, dass die ideale Probekörperlänge unter Abhängigkeit des zuvor festgelegten Radius ermittelt worden ist. Alle Untersuchungen und Schlussfolgerungen die hier angestellt wurden, lassen sich problemlos auf andere Längen-Durchmesser-Verhältnisse übertragen. Beispielsweise wäre es denkbar, dass komplette Probekörper-Setup zu miniaturisieren, um damit homogene Alterungsversuche im Druckbereich zu ermöglichen (vgl. Abbildung A.6).

\subsubsection{Formoptimierung der Halterungskontur}

Aufbauend auf den Erkenntnissen aus der Optimierung der Probekörperlänge soll nun die ideale Halterungskontur ermittelt werden. Dazu wird mit Hilfe der Zielfunktion (Gleichung (3-49) die Halterungskontur in der Weise optimiert, dass sie ideale Eigenschaften bezüglich auftretender Fehlergrößen und Stabilität besitzt. Die Formoptimierung der Halterungskontur wird hier exemplarisch für die Mittelteillänge $l_{0}=50 \mathrm{~mm}$ durchgeführt. Der Grund für die Wahl dieser Länge ist, dass für die ideale Probekörperlänge aus 3.3 .5 keine neue Vulkanisationsform zur Verfügung steht. Da auf die vorhandene Vulkanisationsform (Mittelteillänge $l_{0}=50 \mathrm{~mm}$ ) zurückgegriffen werden muss, soll auch die Formoptimierung der Halterungskontur auf diese Länge angepasst werden. In der Abbildung 3.27 ist der Ablaufplan zur Ermittlung der idealen Halterungskontur dargestellt. Der Ablaufplan ähnelt in seiner Grundstruktur dem der Optimierung der Probekörperlänge. Da sich viele Schritte ähneln, soll hier insbesondere auf die Modifikationen eingegangen werden. Der erste Schritt besteht in der Wahl einer geeigneten Eigenfrequenzvorgabe zur Berechnung der Starthalterungskontur. Natürlich lässt sich ebenso eine bereits vorhandene Halterungskontur als Startkontur für die Formoptimierung nutzen. Die Kontur wird im Anschluss daran mit einem Polynom 5. Grades approximiert. Dieser Polynomgrad eignet sich sehr gut, um die Halterungskonturen abzubilden. Die Verwendung kleinerer Polynomgrade sollte aus Gründen der Abbildungsgenauigkeit vermieden werden. Bei der Verwendung höherer Polynomgrade (sinnvoll bis 8. Grades) steigt die Abbildungsgenauigkeit nur minimal, außerdem führt dies zwangsweise zu längeren Berechnungszeiten aufgrund der zusätzlich zu optimierenden Parameter. Mit Hilfe der approximierten Halterungskontur kann dann das Fehler- und das Instabilitätsmaß berechnet werden. Mittels dieser Werte und der Gewichtung $\alpha$ lässt sich dann die Zielfunktion bestimmen. Diese kann dann mit der Optimierungstoolbox von Matlab minimiert werden. Für die hier durchgeführte Formoptimierung findet die Simplex-Methode als gradientenfreies Verfahren seine Anwendung. Die Formoptimierung endet, wenn die Toleranz eingehalten ist. In der Abbildung 3.28 sind zum besseren Verständnis einige Iterationsschritte des Simplex-Verfahrens dargestellt. 


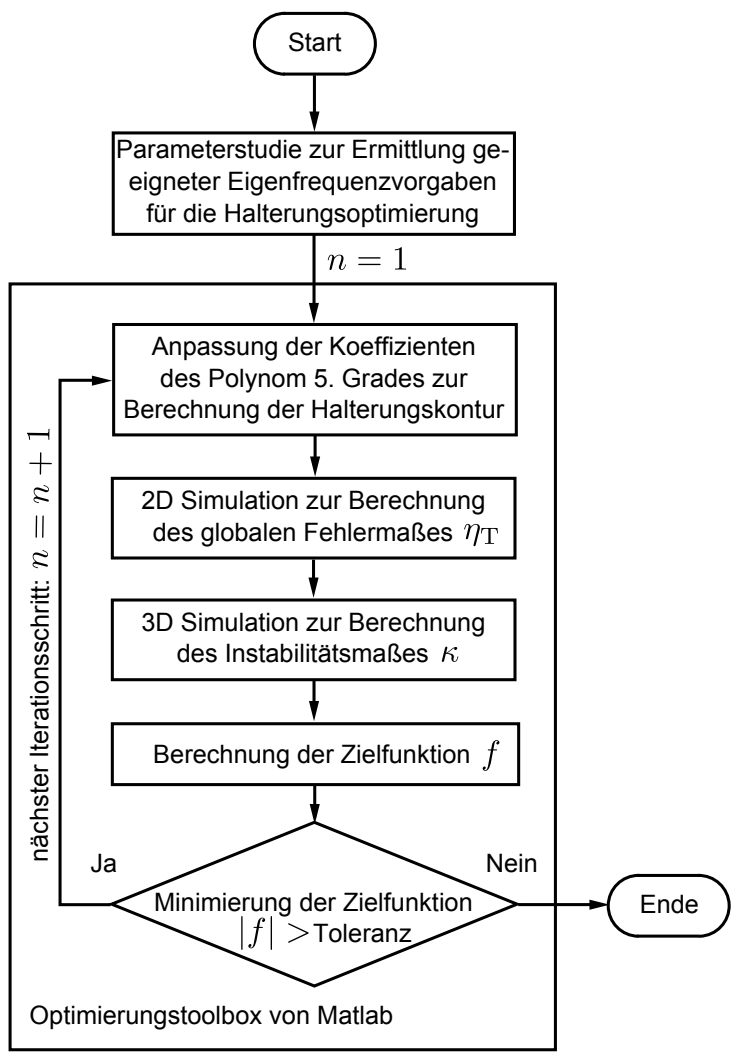

Abb. 3.27 - Ablaufplan für die Formoptimierung der Halterungskontur

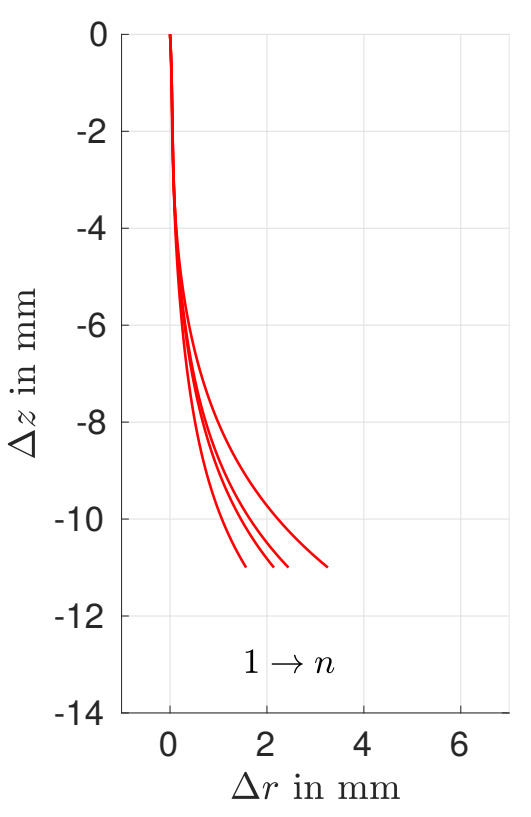

Abb. 3.28 - Iterationsschritte des Simplex-Verfahrens

Zunächst soll anhand zweier Halterungskonturen aus der Parameterstudie, die als Startkonturen dienen sollen, eine Formoptimierung durchgeführt werden. In Abbildung 3.29 sind die Halterungskonturen aus der Parameterstudie zu sehen und in Abbildung 3.30 die Ergebnisse der Formoptimierungen. Dabei stellt die grün-gestrichelte Kontur das Ergebnis dar, welches aus der sicheren Halterungskontur berechnet wurde und die blau-gestrichelte Kontur die Lösung aus der Standard-Halterung. Aus diesen Ergebnissen lassen sich folgende Erkenntnisse gewinnen: Beide Formoptimierungen konvergieren in einen ähnlichen Bereich hinein, liegen jedoch nicht exakt aufeinander. Daraus lässt sich schließen, dass zumindest eine der beiden Konturen in einem lokalen Minimum gelandet ist. Es bleibt zu überprüfen, ob sich das globale Minimum zwischen den Kurven der sicheren und der Standard-Halterung befindet oder sogar zwischen den Kurven der beiden optimierten Lösungen. Die kritische Halterungskontur wurde hier der Vollständigkeit halber mit dargestellt. Da diese Halterungskontur sehr ungünstige Zielfunktionswerte besitzt, sollte sie nicht als Startkontur für eine Optimierung herangezogen werden. Um das Optimierungsproblem zielgerichteter lösen zu können, soll in einem nächsten Schritt ein besser geeigneter Startbereich für die Formoptimierung festgelegt werden. Dazu soll anhand einer Parameterstudie der Eigenfrequenzbereich, der zur Halterungsberechnung dient, abgetastet werden. Mit Hilfe dieser Verläufe kann dann ein idealer Startbereich für die Halterungsoptimierung festgelegt werden. In der Abbildung 3.31 ist der Parameterraum mit den 


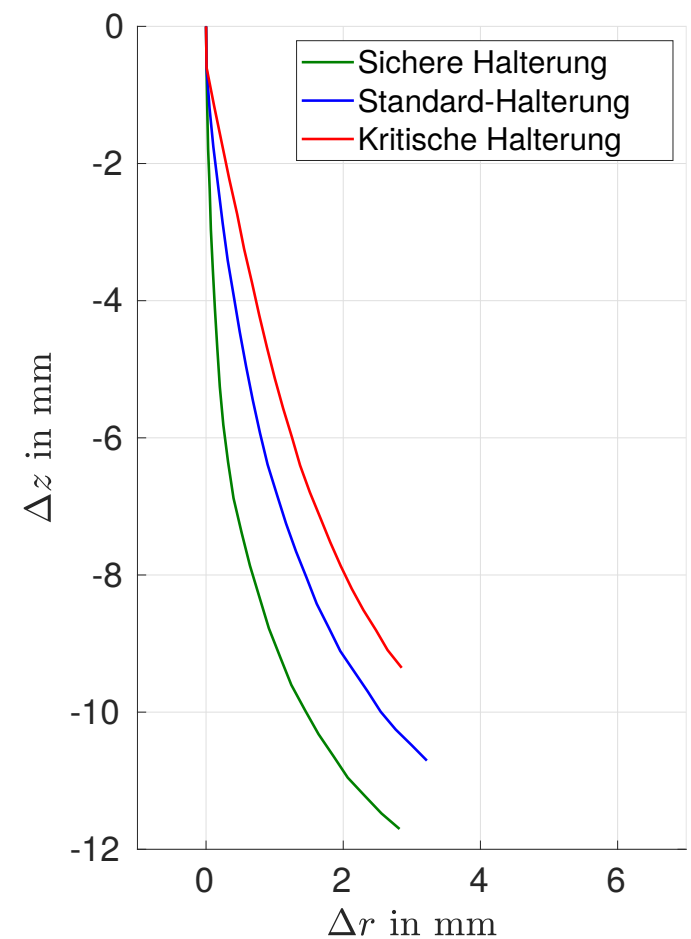

Abb. 3.29 - Halterungen aus der Parameterstudie (Simulationsergebnisse)

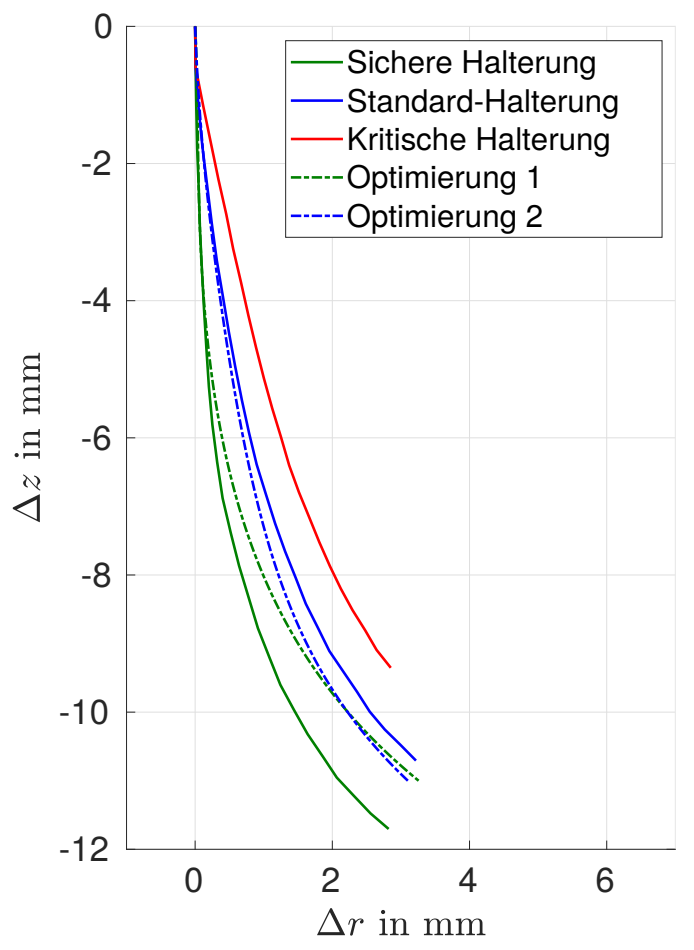

Abb. 3.30 - Lösungen der Optimierung 1 und 2 (Simulationsergebnisse)

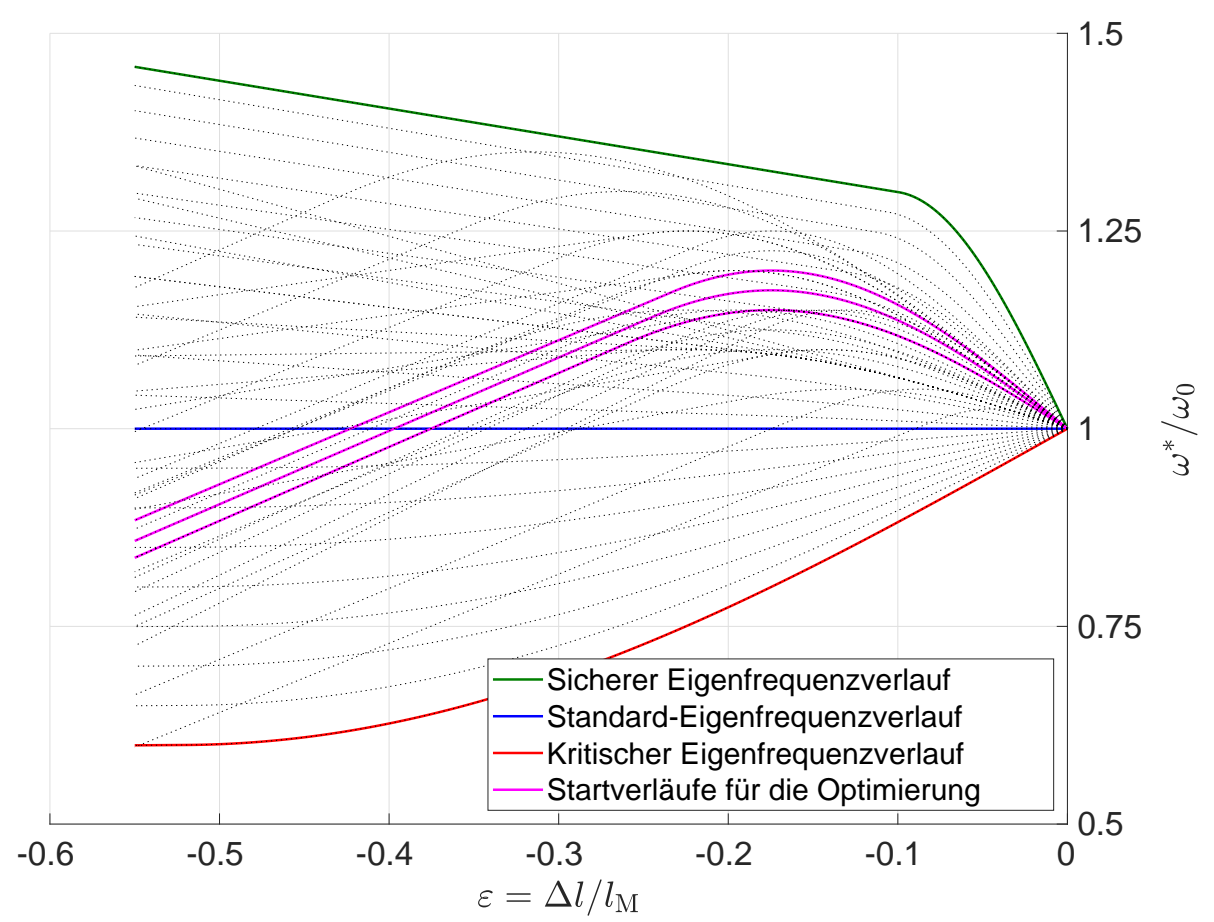

Abb. 3.31 - Normierte Eigenfrequenzverläufe für die Halterungsberechnung (Simulationsergebnisse vgl. Tabelle 3.1. vgl. Abbildung 3.11

normierten Eigenfrequenzverläufen dargestellt. Dabei bilden der kritische und der sichere Eigenfrequenzverlauf die Grenzen für alle weiteren Untersuchungen. Um geeignete Start- 
verläufe zu generieren, wird der Parameterraum hinreichend klein abgetastet, danach die Halterungskontur berechnet und der Zielfunktionswert bestimmt. In Abbildung 3.31 ist die Abtastung anhand der grau hinterlegten Eigenfrequenzverläufe dargestellt. Die Abtastung erfolgte in der Weise, dass Bereiche in denen günstige Zielfunktionswerte auftreten in einem weiteren Schritt nochmals feiner abgetastet wurden. Die in Abbildung 3.31 magentafarben gezeichneten Verläufe stellen die drei ermittelten Minima der Parameterstudie dar. Mit diesen drei Startverläufen sollen im Folgenden die Formoptimierungen erneut durchgeführt werden. In Abbildung 3.32 sind die optimierten Halterungskonturen, die aus

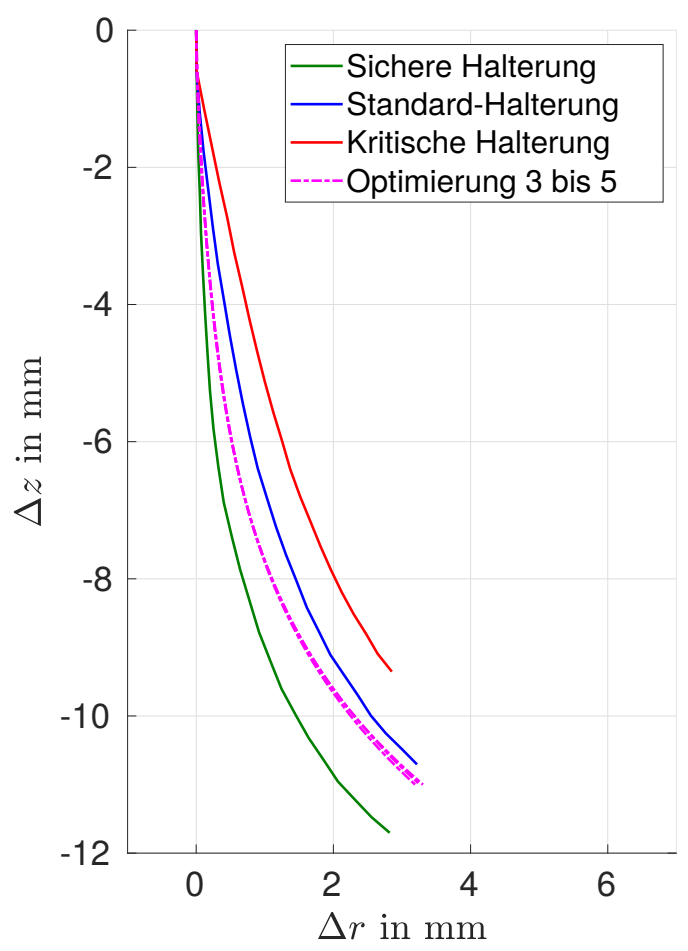

Abb. 3.32 - Lösungen der Optimierung 3 bis 5 (Simulationsergebnisse)

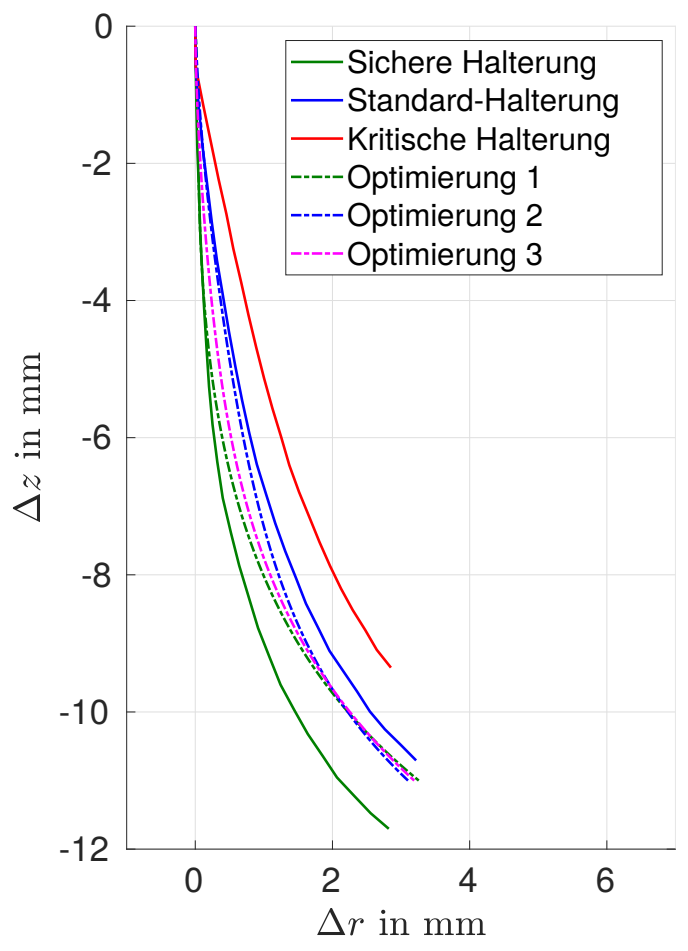

Abb. 3.33 - Gegenüberstellung aller Konturen (Simulationsergebnisse)

den Eigenfrequenzverläufen der Abbildung 3.31 berechnet worden sind, dargestellt. Aus der Abbildung wird ersichtlich, dass die optimierten Verläufe sehr gut übereinander liegen und in ein gemeinsames Minimum konvergieren. Bei Betrachtung der Zielfunktionswerte kann davon ausgegangen werden, dass das globale Minimum eindeutig identifiziert wurde. In Tabelle 3.2 sind die wichtigsten Zielfunktionswerte aus der Halterungsoptimierung zusammengefasst. Anhand der Zielfunktionswerte wird die sehr gute Übereinstimmung der Optimierung 3 bis 5 deutlich. Im Folgenden wird eine der drei optimierten Lösungen mit den Konturen aus der ersten Formoptimierung verglichen. In Abbildung 3.33 ist dieser Vergleich dargestellt. Dabei befindet sich die Kontur mit zugehörigem globalen Minimum der Optimierung innerhalb der Konturen aus den ersten Formoptimierungen. Der untere Abschnitt der Halterungskontur stimmt bei allen Formoptimierungen sehr gut überein. Lediglich im Anfangsbereich der Halterungskonturen sind etwas größere Abweichungen zu erkennen. Ein Grund dafür kann die sehr geringe Sensitivität der Halterung in diesem 


\begin{tabular}{|c|c|c|c|c|}
\hline Halterung & $\eta_{\mathrm{T}}-$ Term & $\kappa-$ Term & Gewichtung $\alpha$ & Zielfunktionswert $f$ \\
\hline Sichere Halterung & 0.017478 & 0.002715 & 0.6 & 0.011573 \\
\hline Standard-Halterung & 0.007549 & 0.012534 & 0.6 & 0.009543 \\
\hline Kritische Halterung & 0.003511 & 0.036936 & 0.6 & 0.016881 \\
\hline Optimierung 1 & 0.010923 & 0.006131 & 0.6 & 0.009006 \\
\hline Optimierung 2 & 0.009273 & 0.009671 & 0.6 & 0.009432 \\
\hline Optimierung 3 & 0.009682 & 0.007269 & 0.6 & 0.008717 \\
\hline Optimierung 4 & 0.009816 & 0.007178 & 0.6 & 0.008761 \\
\hline Optimierung 5 & 0.009867 & 0.007084 & 0.6 & 0.008753 \\
\hline
\end{tabular}

Tabelle 3.2 - Gegenüberstellung der Zielfunktionswerte aus der Halterungsoptimierung

Bereich sein. Die Zielfunktion, die das Maß des relativen Messfehlers und der Instabilität aufsummiert, hat in diesem anfänglichen Bereich nur sehr geringe Funktionswerte. Eine logarithmische Verteilung der Stützstellen der Halterungskontur führte zu keiner nennenswerten Verbesserung. Das bedeutet, dass die Halterungskontur und der sich daraus ergebende Zielfunktionswert nur minimal ändern. Um ein besseres Verständnis für die optimierte Halterungskontur zu bekommen, soll im Weiteren das globale Fehlermaß und Instabilitätsmaß mit den bisherigen Halterungen verglichen werden. In Abbildung 3.34 und Abbildung 3.35 sind die entsprechenden Kurven dargestellt. Aus Abbildung 3.34 lässt

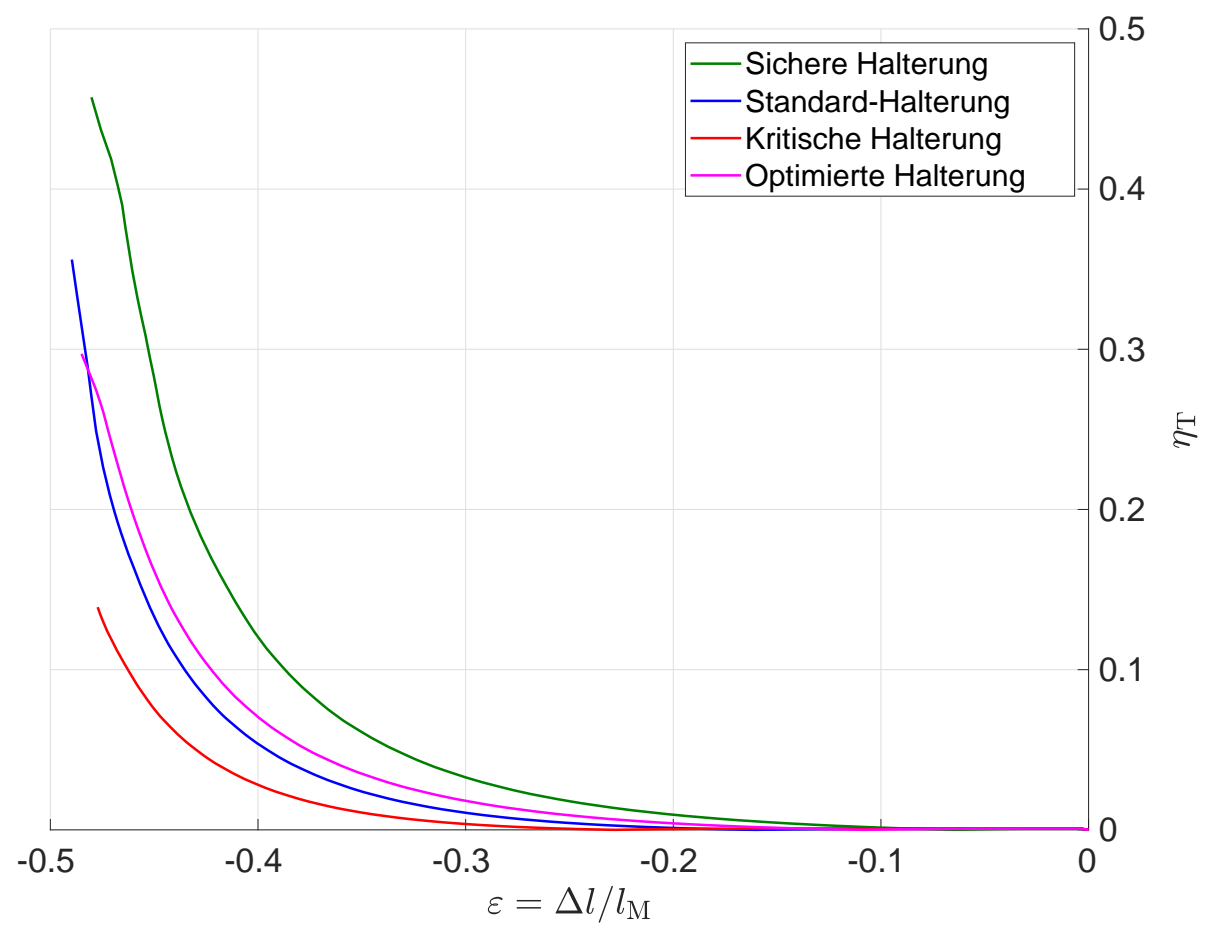

Abb. 3.34 - Darstellung des globalen Fehlermaßes $\eta_{\mathrm{T}}$ für die verschiedenen Halterungskonturen (Simulationsergebnisse vgl. Tabelle 3.1. vgl. Abbildung 3.14 


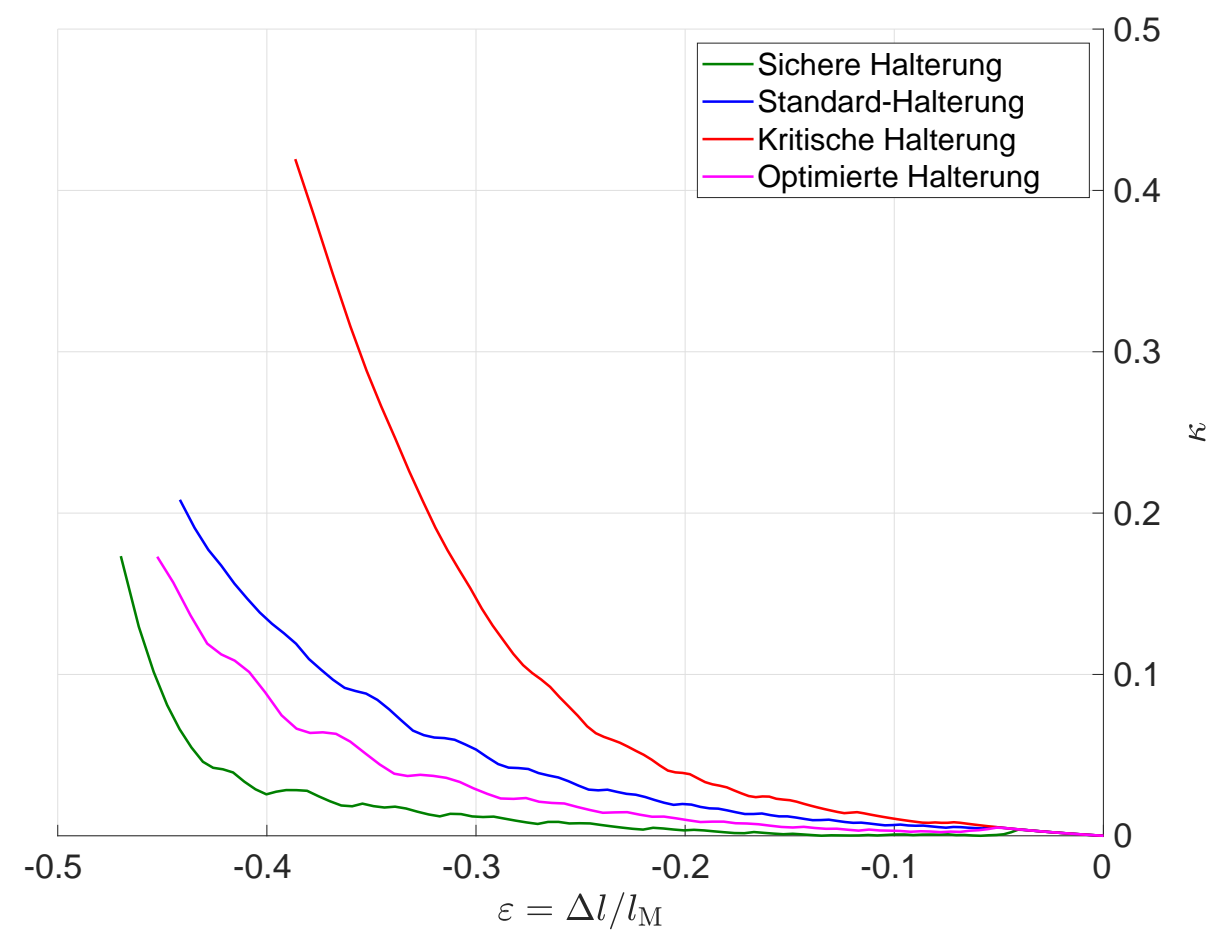

Abb. 3.35 - Darstellung des Instabilitätsmaßes $\kappa$ für die verschiedenen Halterungskonturen (Simulationsergebnisse vgl. Tabelle 3.1. vgl. Abbildung 3.17)

sich entnehmen, dass die optimierte Halterung qualitativ zu ähnlichen Ergebnissen wie die Standard-Halterung führt, aber deutlich bessere Werte als die sichere Halterung liefert. In Abbildung 3.35 befindet sich die optimierte Halterung zwischen der sicheren und der Standard-Halterung, besitzt aber in der Gesamtheit die beste Performance bezüglich Stabilität und Abweichung zur theoretisch vorhandenen Lösung. In Abschnitt 3.4 erfolgt die experimentelle Validierung und Gegenüberstellung der verschiedenen Halterungskonturen.

Abschließend soll der Ablaufplan für die Formoptimierung der Halterungskontur auch für andere Mittelteillängen angewendet werden. Ziel der Untersuchung ist, dass überprüft werden soll, wie gut der Zielfunktionsverlauf der Abbildung 3.25 mit den Lösungen der Formoptimierungen übereinstimmt. In Abbildung 3.36 ist der Zielfunktionsverlauf mit ausgewählten Lösungen der Formoptimierungen (magentafarbene Kreuze) dargestellt. Aus der Abbildung wird ersichtlich, dass die optimierten Halterungskonturen zwar zu besseren Ergebnissen führen, der prinzipielle Zielfunktionsverlauf in seiner Charakteristik aber erhalten bleibt. Das bedeutet, dass die Vorgehensweise, die zur Ermittlung der idealen Halterungskontur angewendet wurde, mit diesen Ergebnissen bestätigt werden kann.

\section{Zusammenfassung der Halterungsoptimierung:}

Als eines der wichtigsten Erkenntnisse kann zusammengefasst werden, dass sich mit Hilfe von Eigenfrequenzverläufen Halterungsgeometrien berechnen und optimieren lassen. Anhand des Ablaufplanes 3.27 kann eine Formoptimierung zur Berechnung der idealen Hal- 


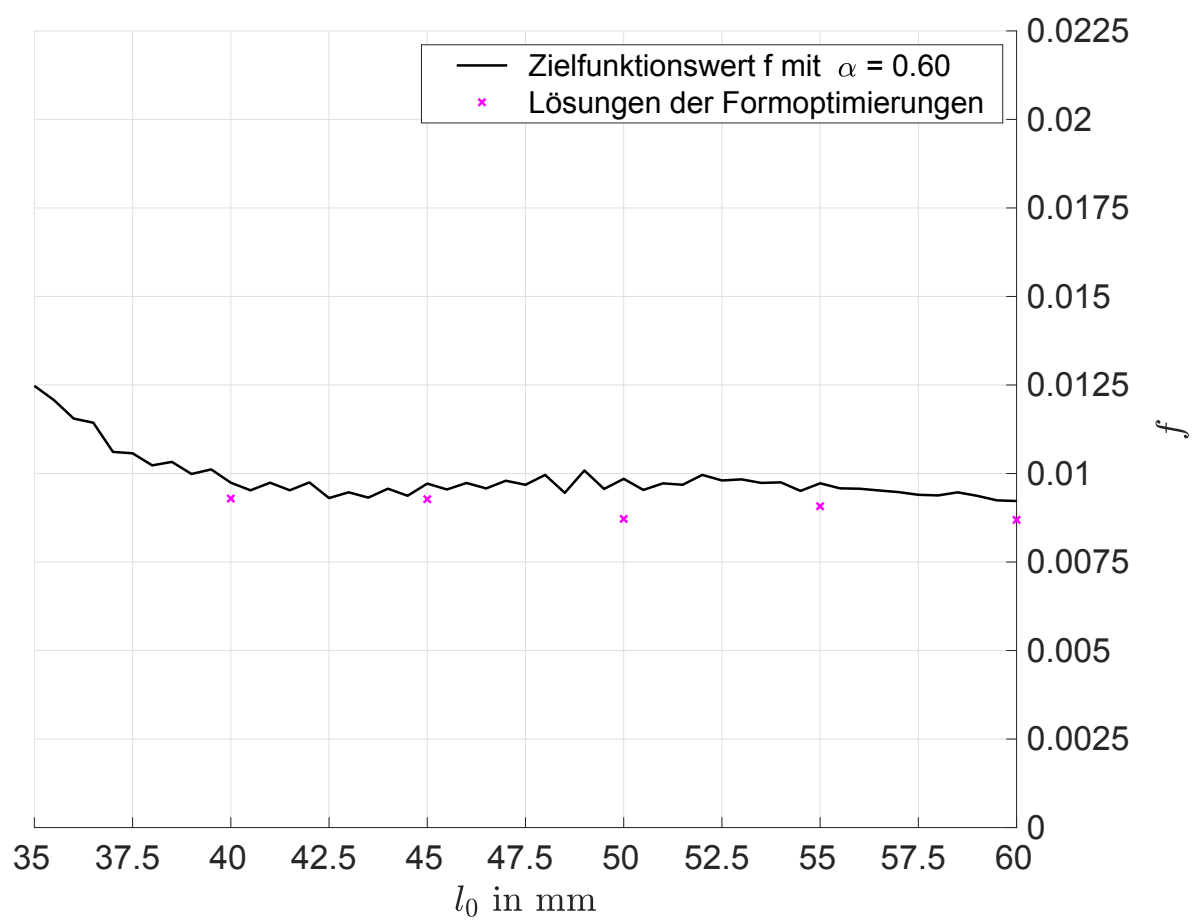

Abb. 3.36 - Vergleich der Zielfunktion für identische asymptotische Eigenfrequenzvorgaben mit ausgewählten Lösungen der Formoptimierungen (Simulationsergebnisse vgl. Tabelle 3.1. vgl. Abbildung 3.25

terungskontur durchgeführt werden. Dabei sollte darauf geachtet werden, dass geeignete Startverläufe für die Optimierung ausgewählt werden. Mittels einer vorgeschalteten Parameterstudie (vgl. dazu Abbildung 3.31) lässt sich die Halterungskontur eindeutig identifizieren. Obwohl die verwendeten Eigenfrequenzverläufe ganz unterschiedliche Formen annehmen, zeigen die zugehörigen Halterungskonturen quantitativ sehr ähnliche Verläufe. Diese Verläufe lassen sich wiederum mittels Polynomen gut approximieren und optimieren. Das hier dargestellte Verfahren lässt sich für beliebige Mittelteillängen anwenden und wurde hier beispielhaft für die Mittelteillänge $l_{0}=50 \mathrm{~mm}$ durchgeführt.

\subsubsection{Erweiterung des Probekörperdesigns für extreme Stauchungen}

Im Folgenden soll für spezielle Anwendungen das Probekörperdesign auf extreme Stauchungen (bis zu $70 \%$ ) erweitert werden. Dazu soll zunächst auf einige prinzipielle Herausforderungen, mit denen bei extremen Stauchungen zu rechnen ist, näher eingegangen werden:

- Für extreme Stauchungen werden längere Halterungskonturen benötigt.

- Der zur Verfügung stehende Messbereich wird immer kleiner.

- Es muss mit zunehmenden Fehlergrößen im Messbereich gerechnet werden. 
- Die Abweichung vom einachsigen Spannungszustand wird mit zunehmender Stauchung immer größer.

Die Reduktion des Messbereiches führt dazu, dass die Messstrecke $l_{\mathrm{M}}$ ab einer gewissen Stauchung von der Halterungskontur verdeckt wird. Eine mögliche Idee bestünde in der Verkleinerung der Messstrecke $l_{\mathrm{M}}$, damit diese erst zu einem späteren Zeitpunkt mit der Halterung in Kontakt tritt. In Abbildung 3.37 ist die axiale Dehnung für verschiedene Messlängen $l_{\mathrm{M}}$ dargestellt. Die Dehnungsverläufe $\left(l_{\mathrm{M}}=20 \mathrm{~mm}\right.$ und $\left.l_{\mathrm{M}}=15 \mathrm{~mm}\right)$ sind bis

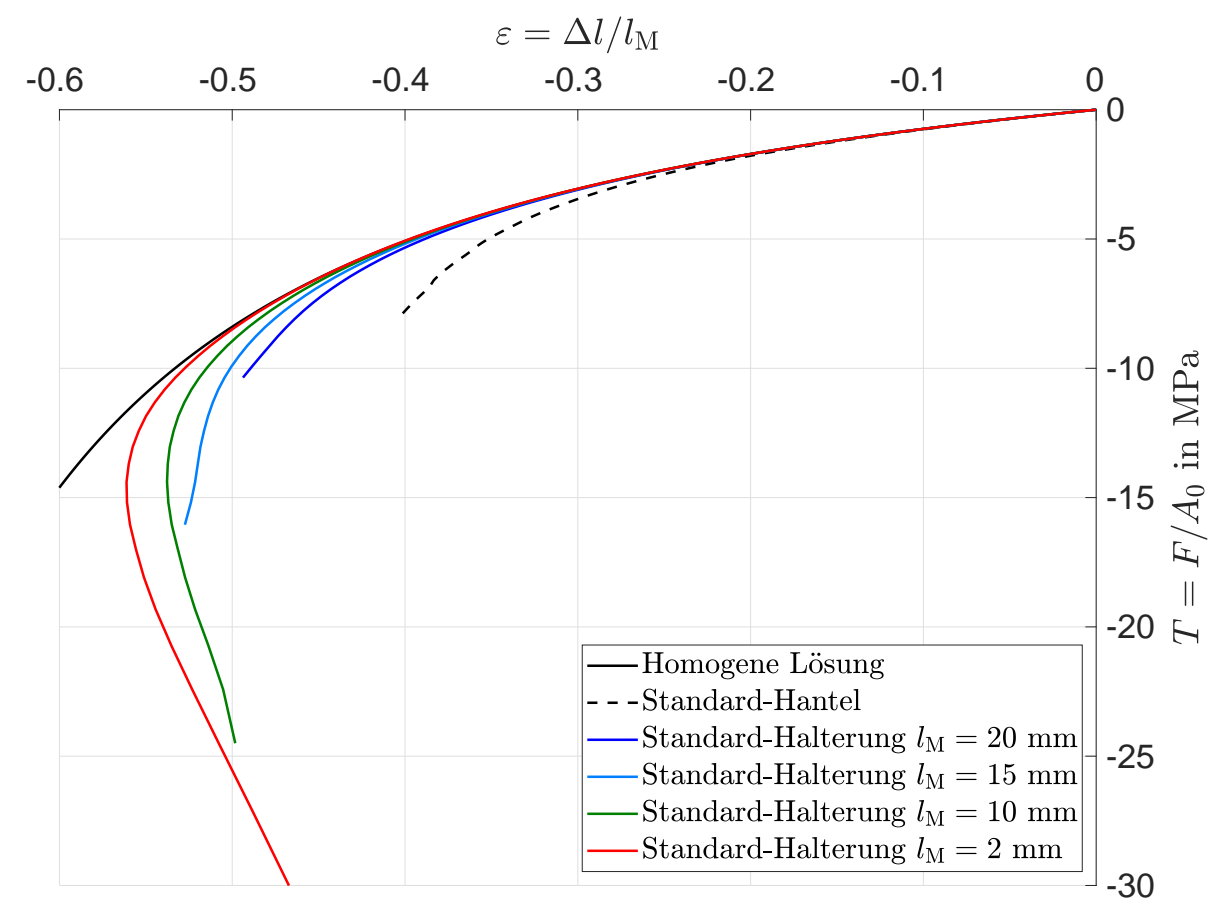

Abb. 3.37 - Darstellung der axialen Dehnungsmessung für unterschiedliche Messstrecken $l_{\mathrm{M}}$ (Simulationsergebnisse vgl. Tabelle 3.1. vgl. Abbildung 3.13)

zu der Stauchung dargestellt, bei der eine Kontaktierung mit der Halterung stattfinden würde. Anhand der Abbildung 3.37 wird weiterhin deutlich, dass eine Verkleinerung der Messlänge zwar prinzipiell eine Messung hin zu größeren Stauchungen ermöglicht, aber selbst diese Herangehensweise ihre Grenzen aufzeigt. Für die Messlänge $l_{\mathrm{M}}=10 \mathrm{~mm}$ und $l_{\mathrm{M}}=2 \mathrm{~mm}$ ist interessanterweise sogar ein Richtungswechsel der Dehnungskurven zu verzeichnen. Dieser Effekt lässt sich dadurch erklären, dass bei extremen Druckbeanspruchungen das Material immer stärker in radialer Richtung aufgeweitet wird und dadurch keine reine Stauchung in axialer Richtung an der Probenoberfläche mehr stattfindet. Ein weiterer Nachteil in der Reduzierung der Messstrecke liegt in der Vergrößerung des relativen Messfehlers. Die direkte axiale Dehnungsmessung scheint unter den gegebenen Gesichtspunkten ihre Grenzen zu erreichen.

Im Folgenden soll ein alternativer Zugang zur Messung der Dehnungen (in der Symmetrieebene) vorgestellt werden. Der Kerngedanke der neuen Messstrategie besteht darin, über die Messung der Umfangsstreckung auf eine gemittelte Streckung in axialer Rich- 
tung schließen zu können. Dieser Ansatz, der auf M. Sc. Schlomka und M. Sc. Gelke an der Professur Festkörpermechanik zurückzuführen ist (vgl. Kanzenbach u.a. 2019b), soll im Weiteren näher betrachtet und auf seine Anwendungsmöglichkeiten hin getestet werden. Zur Herleitung der neuen Messstrategie wird zunächst das Volumen einer Materialschicht infinitesimaler Höhe in der Symmetrieebene betrachtet. Als nächstes wird das Volumenverhältnis zwischen verformter und unverformter Lage aufgestellt (in der unverformten Lage kann die infinitesimale Höhe $\widetilde{h}$ als konstant angesehen werden):

$$
\frac{V}{\widetilde{V}}=\frac{\int h(r) \mathrm{d} A}{\widetilde{h} \widetilde{A}}=\frac{1}{\widetilde{A}} \int \frac{h(r)}{\widetilde{h}} \mathrm{~d} A .
$$

Hierbei lassen sich bekannte Größen wie folgt durch Streckungen ersetzen:

$$
\frac{h(r)}{\widetilde{h}}=\lambda_{z} \quad \text { und } \quad \frac{A}{\widetilde{A}}=\frac{d^{2}}{\widetilde{d}^{2}}=\lambda_{u}^{2}
$$

Über die Definition der gemittelten axialen Streckung $\lambda_{z, \mathrm{~m}}$ ergibt sich dann folgender Zusammenhang:

$$
\frac{V}{\widetilde{V}}=\lambda_{u}^{2} \lambda_{z, \mathrm{~m}} \quad \text { mit: } \quad \lambda_{z, \mathrm{~m}}=\frac{1}{A} \int \lambda_{z} \mathrm{~d} A
$$

Für den Fall idealer Inkompressibilität $\frac{\mathrm{d} V}{\mathrm{~d} \widetilde{V}}=J_{3} \stackrel{!}{=} 1$ kann die Auswertegleichung (3-52) weiter vereinfacht werden:

$$
\lambda_{z, \mathrm{~m}}=\frac{1}{\lambda_{u}^{2}}
$$

Zum besseren Vergleich mit der herkömmlichen Dehnungsmessung via Extensometer soll die gemittelte axiale Streckung als technische Dehnung dargestellt werden:

$$
\varepsilon_{z z, \mathrm{~m}}=\lambda_{z, \mathrm{~m}}-1=\frac{1}{\lambda_{u}^{2}}-1=\left(\frac{\widetilde{d}}{d}\right)^{2}-1
$$

Abschließend soll das hergeleitete Dehnungsmaß in Gleichung 3-54 numerisch überprüft werden. Hierfür wird der rechte Ausdruck der Gleichung 3-52 hinreichend fein diskretisiert mittels einer gewichteten axialen Dehnungsmessung in der Symmetrieebene. Die dabei vorgenommene Gewichtung erfolgt in der Weise, dass die axialen Dehnungen in radialer Richtung aufsummiert und anhand der zugehörigen Kreisringfläche gemittelt werden:

$$
\varepsilon_{z z, \mathrm{M}}=\frac{\frac{1}{2} \sum_{i=1}^{n}\left(\varepsilon_{i+1}+\varepsilon_{i}\right)\left(r_{i+1}^{2}-r_{i}^{2}\right)}{r_{\max }^{2}} .
$$

In Abbildung 3.38 werden die verschiedenen Dehnungsvarianten gegenübergestellt und mit der homogenen Lösung verglichen. Anhand der Abbildung wird deutlich, dass die 


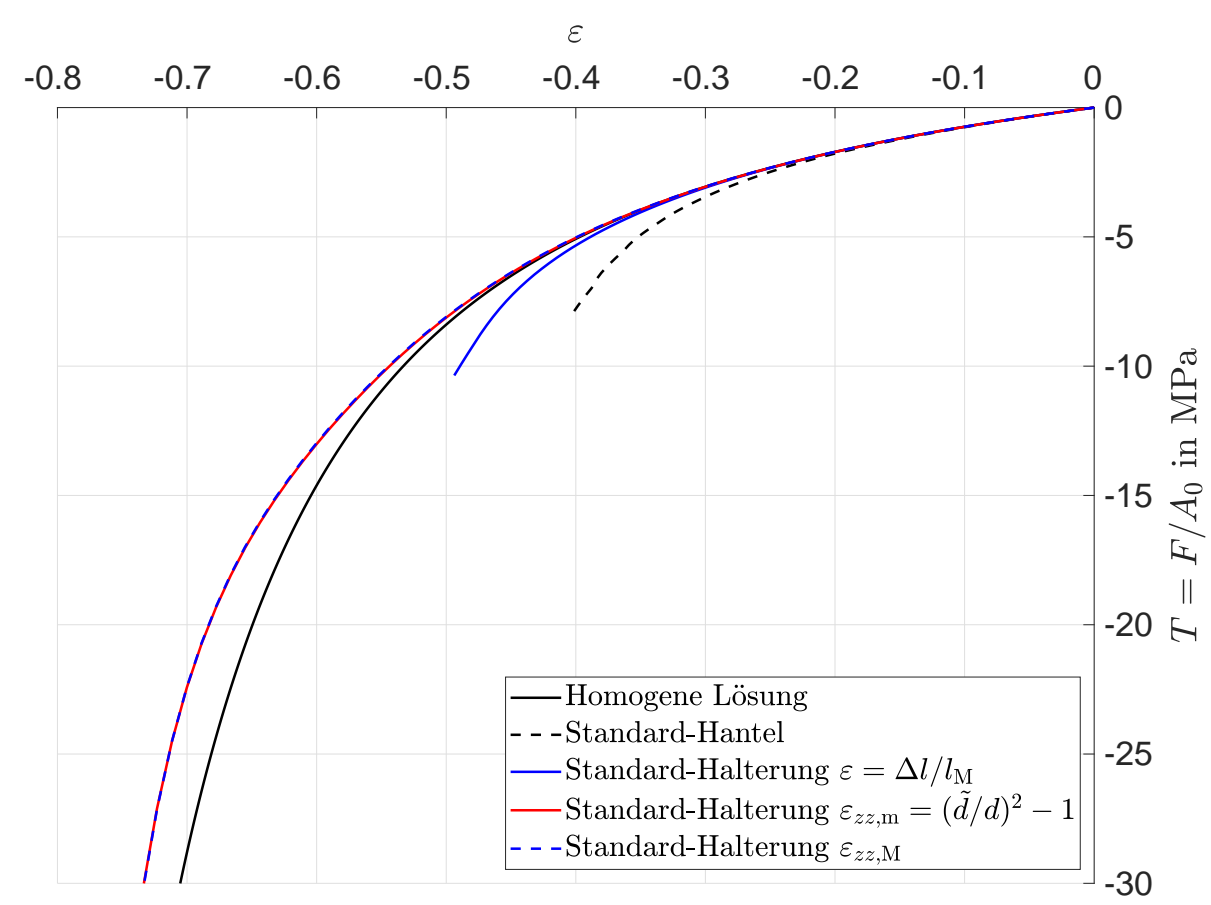

Abb. 3.38 - Vergleich der verschiedenen Messstrategien (Simulationsergebnisse vgl. Tabelle 3.1. vgl. Abbildung 3.13 die letzten beiden Kurven fallen zusammen)

neue Messstrategie (Messung der Umfangsstreckung) zu übereinstimmenden Ergebnissen führt wie die Mittelung der Dehnungen in radialer Richtung über die jeweiligen Kreisringflächen, siehe Gleichung (3-55). Um diesen Sachverhalt noch eingehender untersuchen zu können, soll im Folgenden der Verlauf der axialen Dehnungen in der Symmetrieebene in radialer Richtung dargestellt werden, siehe Abbildung 3.39. Weiterhin ist in der Abbildung der Mittelwert der axialen Dehnung (Gleichung (3-55p) und das Ergebnis der neuen Messstrategie dargestellt. Als eine Erkenntnis kann festgehalten werden, dass mit zunehmender Stauchung (hier exemplarisch für: 50 \%) der Verlauf der axialen Dehnung in radialer Richtung immer inhomogener wird. Weiterhin kann bestätigt werden, dass die neue Messstrategie einen indirekten Zugang liefert, um bei großen bzw. extremen Stauchungen (bis $70 \%$ ) messen zu können.

Die größer werdenden Abweichungen von der homogenen Lösung im Spannungs-Dehnungsdiagramm (Abbildung 3.38) lassen sich dadurch erklären, dass bei großen Stauchungen die Annahme einer homogenen Druckdeformation immer stärker verletzt wird. Das äußert sich auch im inhomogenen Verlauf der axialen Dehnung in radialer Richtung. An dieser Stelle sei aber nochmal ausdrücklich auf den Umstand hingewiesen, dass die neue Messstrategie überhaupt einen Zugang liefert, um bei solchen extremen Stauchungen messen zu können. Abgesehen davon soll auf den großen messtechnischen Vorteil eingegangen werden, dass die neue Messstrategie (Messung der Umfangsstreckung in horizontaler Richtung) sehr platzsparend auf einen kleinen Messbereich zwischen den Halterungskonturen angewandt 


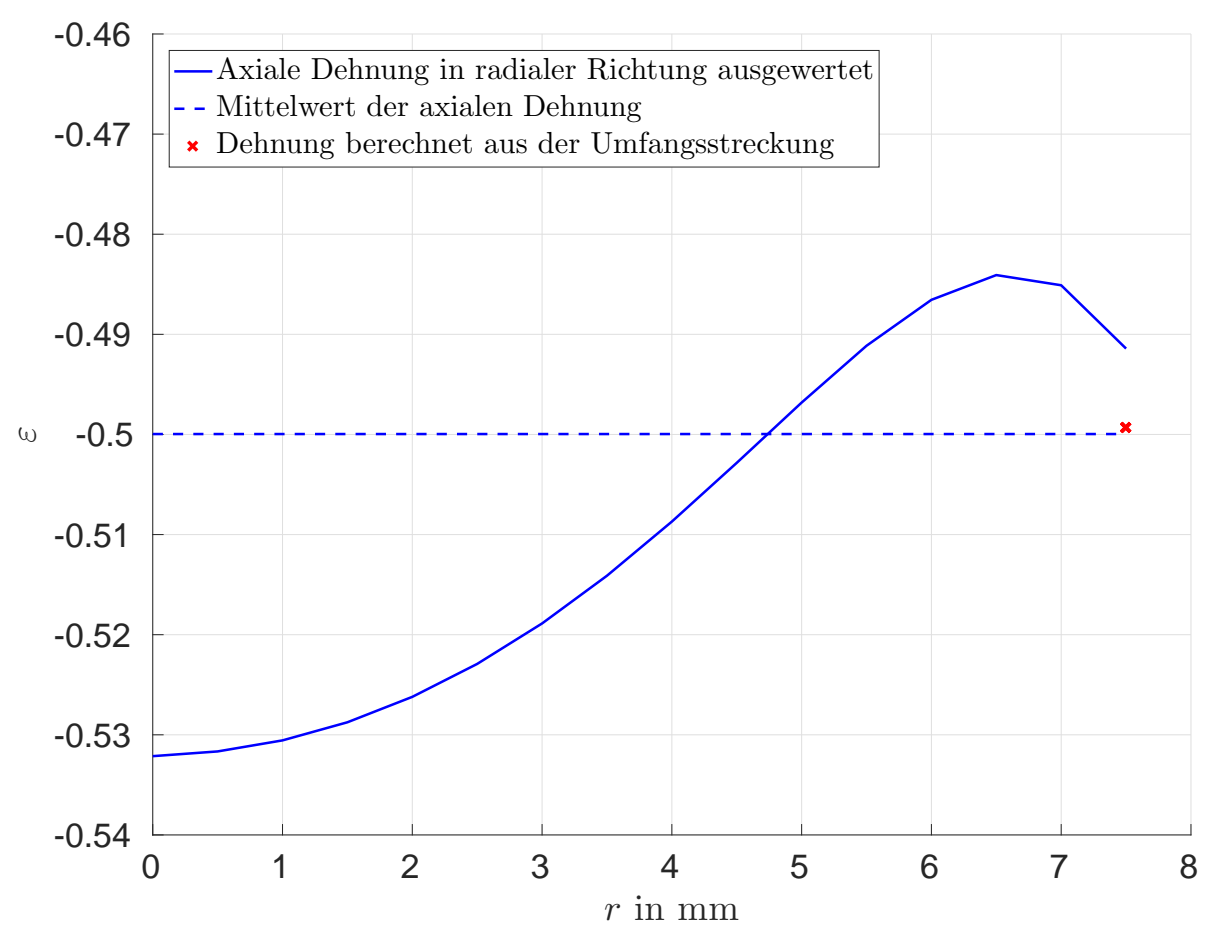

Abb. 3.39 - Auswertung der axialen Dehnungen in radialer Richtung für den Querschnitt in der Symmetrieebene (Simulationsergebnisse vgl. Tabelle 3.1)

werden kann (vgl. Gleichung (3-54)).

\section{Untersuchung der Anwendungsmöglichkeiten und Fehlergrenzen der neuen Messstrategie}

Im Weiteren soll die neue Messstrategie auf ihre Anwendungsmöglichkeiten und Grenzen hin getestet werden. Dabei soll auch der Fehler untersucht werden, der entsteht, wenn die Annahmen, die bei der Herleitung der neuen Messstrategie getroffen wurden, nur näherungsweise erfüllt sind:

- Anwendung der neuen Messstrategie außerhalb der Symmetrieebene

- Einfluss der Reibung und des Kompressionsmoduls auf die Auswertegleichung

- Einfluss einer gestörten Kreisgeometrie des Querschnitts auf die Auswertegleichung Zunächst soll überprüft werden, wie sensitiv die neue Messstrategie darauf reagiert, wenn außerhalb der Symmetrieebene gemessen wird. Da der zur Verfügung stehende Messbereich für extreme Stauchungen sehr begrenzt ist (hier: $3.2 \mathrm{~mm}$ ), reicht es die Untersuchungen für einen Offset von $\Delta z=2 \mathrm{~mm}$ zur Symmetrieebene durchzuführen. In Abbildung 3.40 sind die relativen Fehlerkurven, verglichen mit der idealen Lösung bei $\Delta z=0 \mathrm{~mm}$, dargestellt. Aus Abbildung 3.40 wird ersichtlich, dass der maximale Messfehler im Bereich von 0 bis $70 \%$ Stauchung bei unter $f<0.4 \%$ liegt. Das Minimum der Fehlerkurven bei ca. $57 \%$ Stauchung lässt sich dadurch erklären, dass die axialen Dehnungen in Oberflächennähe infolge der Materialauswölbung beim Übergang zu extremen Stauchungen einen Umkehrpunkt durchlaufen (vgl. Abbildung 3.37). Zum besseren Verständnis ist dazu in Abbildung 


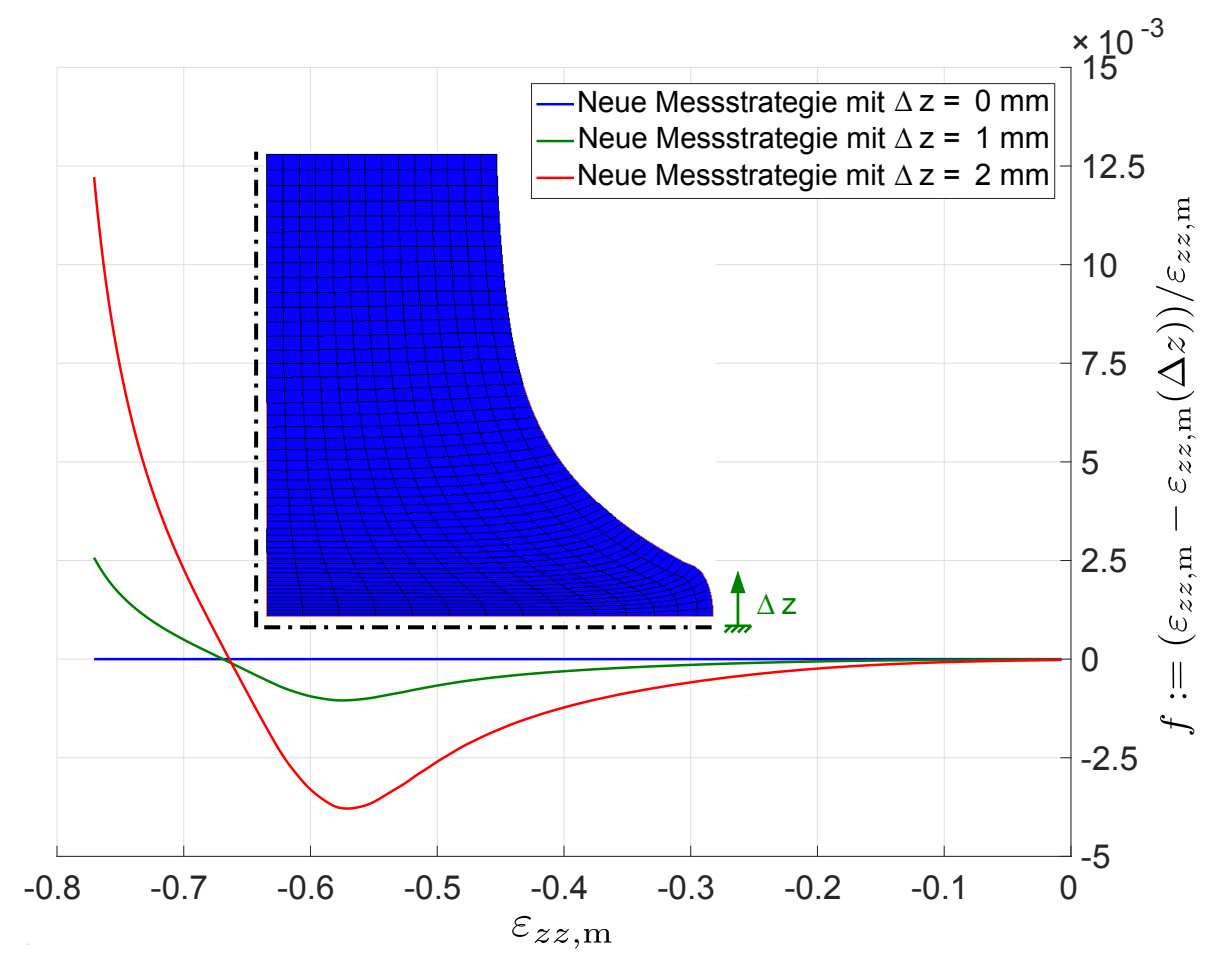

Abb. 3.40 - Darstellung des relativen Messfehlers $f$ bei Messung außerhalb der Symmetrieebene mit der neuen Messstrategie (Simulationsergebnisse vgl. Tabelle 3.1

3.40 ein Symmetrieschnitt des Probekörpers dargestellt. Betrachtet man die unterste Elementschicht in radialer Richtung, dann wird anhand der im unverformten Zustand horizontalen Gitterlinien deutlich, dass die äußeren Elemente immer stärker aufgeweitet statt komprimiert werden. Zusammenfassend lässt sich sagen, dass auch ein minimaler Offset von wenigen Millimetern nicht zu signifikant schlechteren Messergebnissen führt und die neue Messstrategie auch dafür geeignet ist.

Als nächstes soll der Einfluss der Reibung und des Kompressionsmoduls auf die neue Messstrategie hin untersucht werden. Es soll dabei überprüft werden, ob die Annahme der Inkompressibilität für die neue Messstrategie zulässig ist oder ob eine schwache Kompressibilität berücksichtigt werden muss. In den Veröffentlichungen von Stommel \& Zimmermann (2011), Zimmermann \& Stommel (2013) und Kanzenbach u.a. (2017) werden verschiedene Verfahren zur experimentellen Bestimmung des Kompressionsmoduls technischer Elastomere vorgestellt. Der darin ermittelte Kompressionsmodul liegt in der Größenordnung von $2500 \mathrm{MPa}$. Für die hier numerisch durchgeführten Untersuchungen wird der Kompressionsmodul im Bereich von $K \in[100,10000]$ MPa variiert. Somit kann die neue Messstrategie für diesen Bereich hinreichend getestet werden. Die Variation der Reibung erfolgt dabei im Bereich von $\mu \in[0,1]$. In Abbildung 3.41 sind die berechneten Dehnungskurven für die Variation des Kompressionsmoduls und der Reibung dargestellt. Im Folgenden soll der relative Fehler der berechneten Dehnungskurven im Vergleich zuein- 


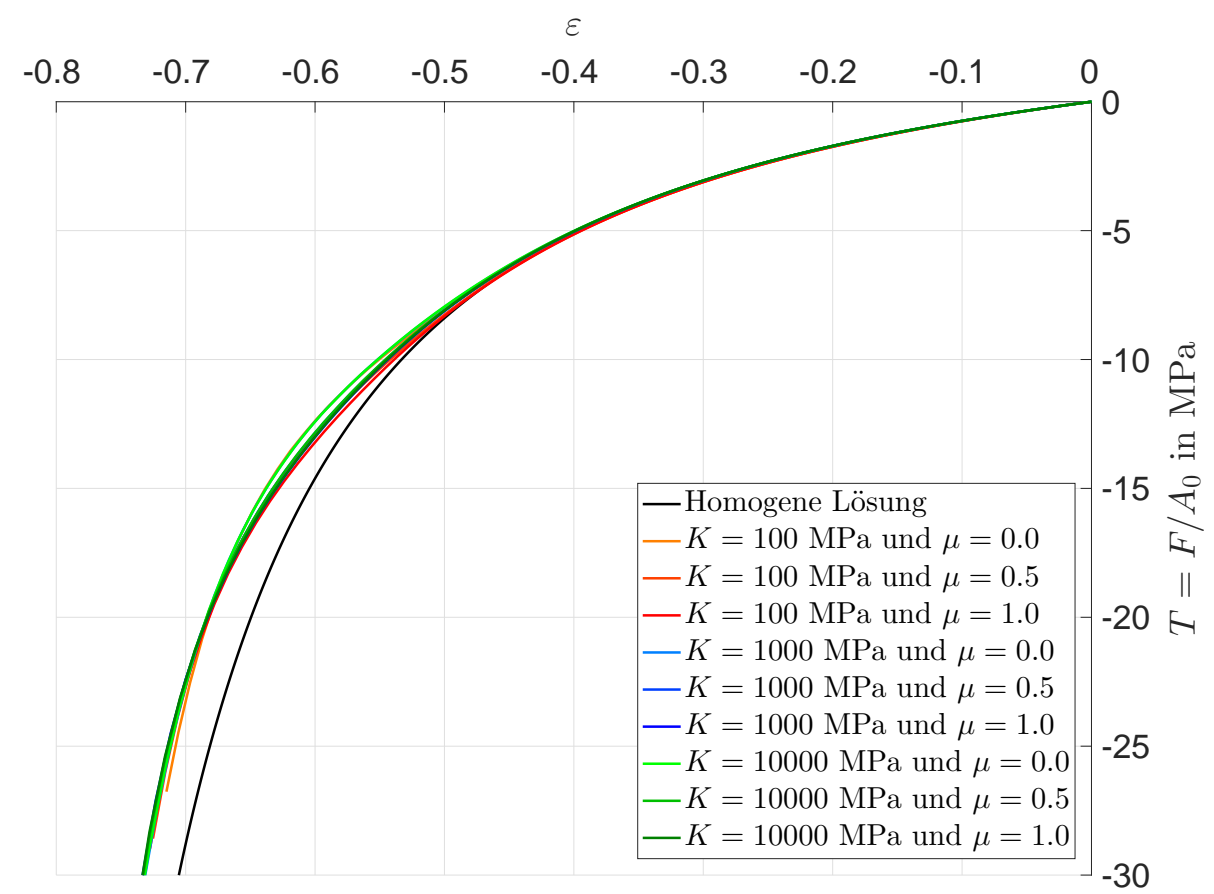

Abb. 3.41 - Überprüfung der neuen Messstrategie bei Variation des Kompressionsmoduls und der Reibung (Simulationsergebnisse vgl. Tabelle 3.1, die farbigen Kurven überlagern sich)

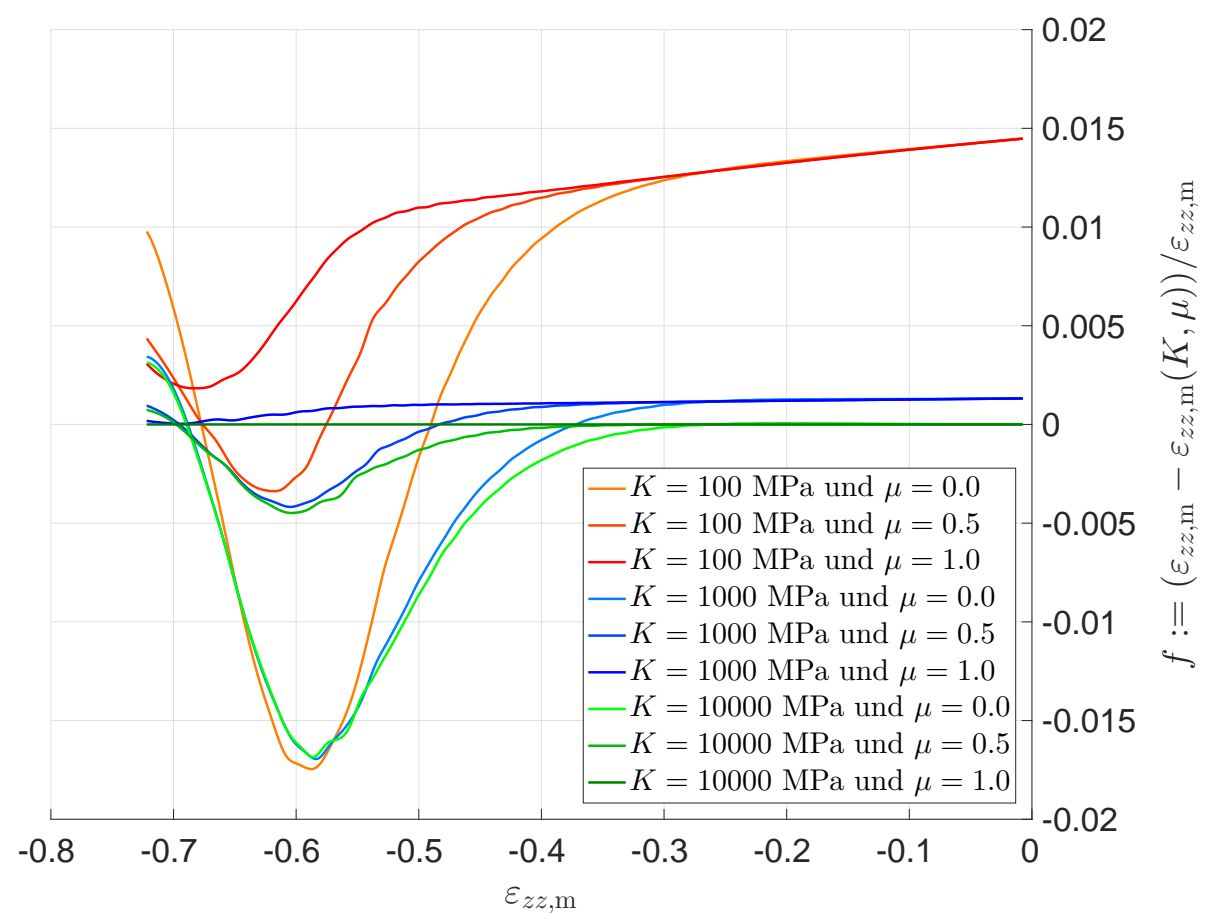

Abb. 3.42 - Darstellung des relativen Messfehlers $f$ bei Variation der Reibung und des Kompressionsmoduls (Simulationsergebnisse vgl. Tabelle 3.1

ander ermittelt werden (vgl. Abbildung 3.42). Die Paarung $K=10000 \mathrm{MPa}$ und $\mu=1.0$ (oberer Grenzbereich) stellt dabei eine theoretische Vergleichslösung dar. Aus Abbildung 
3.42 wird ersichtlich, dass die Reibung bis $30 \%$ Stauchung keinen abweichenden Einfluss auf die neue Messstrategie ausübt. Erst bei größeren Stauchungen ist ein abweichendes Reibverhalten zu verzeichnen, was zu einem maximalen Fehler von $3 \%$ führt. Dieser Effekt kann dadurch erklärt werden, dass der Probekörper in radialer Richtung, aufgrund der abflachenden Halterungskontur, immer stärker aufgeweitet wird und dadurch ein Gleiten begünstigt. Die unterschiedlichen Offsets für die Fehlerkurven lassen sich auf den jeweils gewählten Kompressionsmodul zurückführen. Durch die Veränderung des Kompressionsmoduls im hyperelastischen Stoffgesetz wird zwangsweise die Materialsteifigkeit verändert, was sich wiederum auf die Berechnung des relativen Fehlers auswirkt. Der stärkste Einfluss ist dabei für einen Kompressionsmodul von $100 \mathrm{MPa}$ zu verzeichnen. Da dieser Wert für technische Elastomere alles andere als repräsentativ ist, dient er hier lediglich als theoretischer Grenzfall. Hingegen liegt der relative Fehler für Werte des Kompressionsmoduls zwischen 1000 und $10000 \mathrm{MPa}$ durchgehend unter $f<2 \%$ (bei gleichem Reibwert sogar unter $f<0.2 \%$ ). Mit dieser Überprüfung kann sichergestellt werden, dass die Annahme der Inkompressibilität für die neue Messstrategie verwendet werden darf.

Abschließend soll überprüft werden, wie sensitiv sich die neue Messstrategie bei einem unrunden Querschnitt verhält. Die Störung wird dabei in Form einer Geometrieimperfektion $u_{\mathrm{S}}=\iota r_{0}$ aufgebracht mit $\iota \in[0.01,0.02]$ (initiale Auslenkung der Mittelebene um den Betrag $u_{\mathrm{S}}$ ). Neben der Imperfektion wird auch der Schrumpf des Probekörpers variiert $\Delta r \in[1,2] \%$. Der zu ermittelnde relative Fehler $f$ wird dabei aus der gewichteten axialen Dehnungsmessung $\varepsilon_{\mathrm{zz}, \mathrm{M}}$ und der Dehnungsmessung via Umfangsstreckung $\varepsilon_{\mathrm{zz}, \mathrm{m}}$ bestimmt. Aufgrund der Geometrieimperfektion wird die Umfangsstreckung an drei verschiedenen Stellen entlang des Umfangs ermittelt $\varphi \in[0,180]^{\circ}$. Dies bietet den großen Vorteil, dass in direkter Weise überprüft werden kann, wie sich eine Messung mit der neuen Messstrategie in Knickrichtung, $90^{\circ}$ dazu versetzt oder entgegen der Knickrichtung verhält. In der Abbildung 3.43 ist die relative Abweichung über der gewichteten axialen Dehnungsmessung dargestellt. Zunächst wird deutlich, dass eine Geometrieimperfektion einen größeren Einfluss auf das Fehlermaß ausübt als der Schrumpf des Probekörpers. Weiterhin ist zu erkennen, dass die Messfehler in der Knickebene (Messpunkt links: $180^{\circ}$ und Messpunkt rechts: $0^{\circ}$ ) am größten und $90^{\circ}$ dazu versetzt am kleinsten sind. Interessanterweise liegen die Fehlerkurven resultierend aus dem Messpunkt in der Mitte $\left(90^{\circ}\right.$ zur Knickrichtung versetzt) für alle Imperfektionen nahezu aufeinander. Als eine der wichtigsten Erkenntnisse kann festgehalten werden, dass selbst bei einer Geometrieimperfektion von $\iota=0.02$ und einem Schrumpf von $\Delta r=1 \%$ der maximale Messfehler unter $f<3.6 \%$ liegt. Somit ist gewährleistet, dass die neue Messstrategie auch bei kleinen Störungen der Kreisgeometrie zu genauen Ergebnissen führt. Bei einer späteren experimentellen Realisierung sollte trotzdem darauf geachtet werden, dass der Probekörper möglichst koaxial eingebaut und der Schrumpf bestmöglich durch einen entsprechenden Halterungsring kompensiert wird. 


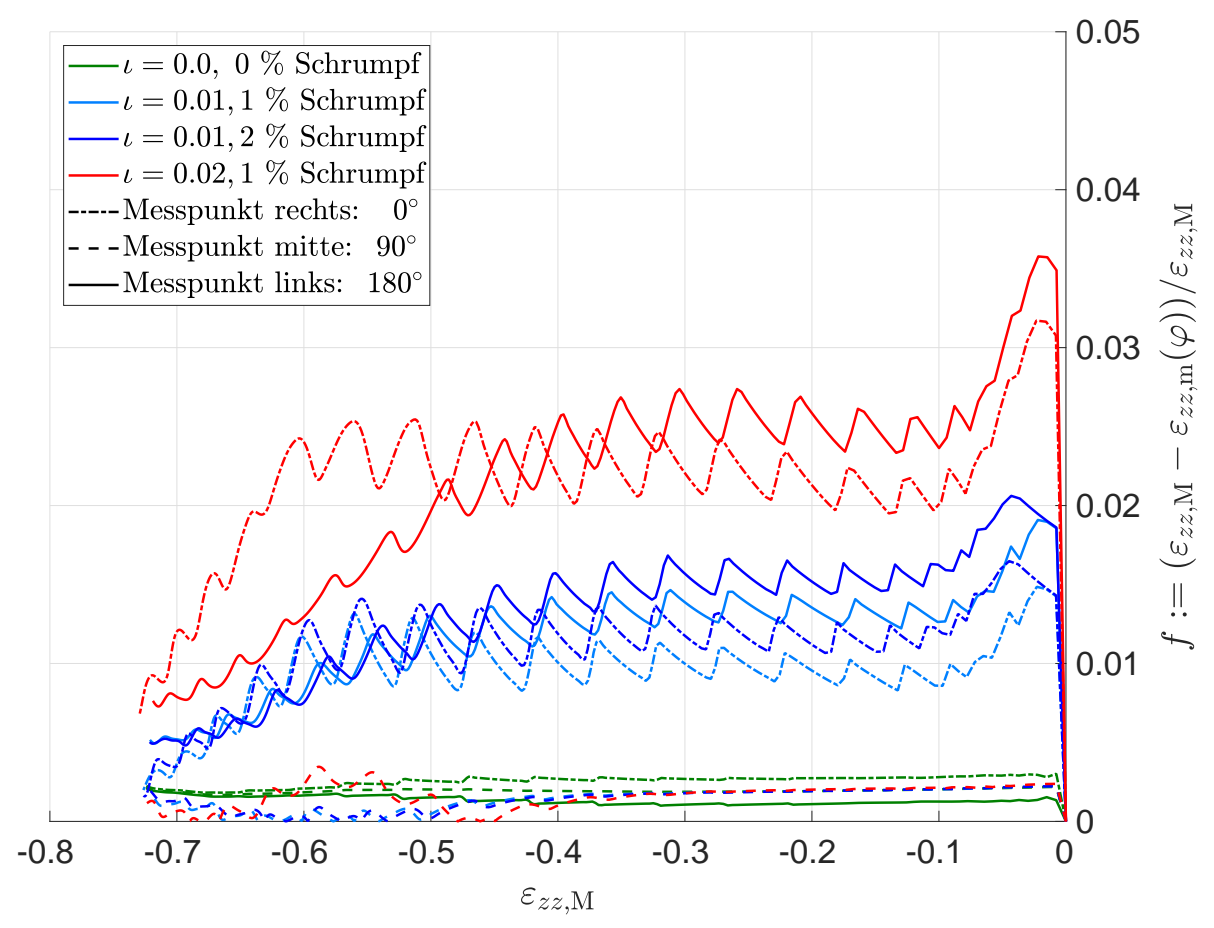

Abb. 3.43 - Darstellung des relativen Messfehlers $f$ für die neue Messstrategie bei unrundem Querschnitt (Simulationsergebnisse vgl. Tabelle 3.1)

\section{Zusammenfassung der neuen Messstrategie:}

In diesem Abschnitt konnte mit Hilfe der neuen Messstrategie (Messung der Umfangsstreckung zur Bestimmung der gemittelten axialen Dehnung) ein Werkzeug bereitgestellt werden, dass es in Kombination mit längeren Halterungskonturen ermöglicht, Stauchungen bis $70 \%$ zu realisieren und zu messen. Da die gemittelte axiale Dehnung nur indirekt über die Umfangsstreckung bestimmt werden kann, wurde eine ausführliche Sensitivitätsanalyse der neuen Messstrategie durchgeführt. Die Untersuchungen haben ergeben, dass selbst bei kleinen Störungen der getroffenen Annahmen die gemittelte axiale Dehnung zuverlässig bestimmt werden kann. Die experimentelle Validierung der neuen Messstrategie erfolgt im letzten Abschnitt von 3.4.4. 


\subsection{Experimentelle Validierung des neuen Probekörper-Setups}

\subsubsection{Versuchsaufbau des Probekörper-Setups in der Prüfmaschine}

In diesem Abschnitt erfolgt die experimentelle Validierung des neuen Probekörper-Setups. Dazu werden die entwickelten Halterungskonturen entsprechend ihrer Geometrie präzise gefertigt und im Anschluss getestet (vgl. die vier Konturen in Abbildung 3.32). Zum besseren Verständnis soll zunächst auf den prinzipiellen Versuchsaufbau eingegangen werden. In Abbildung 3.44 ist der allgemeine Versuchsaufbau mit allen wichtigen Komponenten dargestellt und in Abbildung 3.45 sind die Einzelkomponenten für den Zusammenbau des Probekörper-Setups zu sehen. Die Montage des Probekörper-Setups (1) erfolgt über zwei

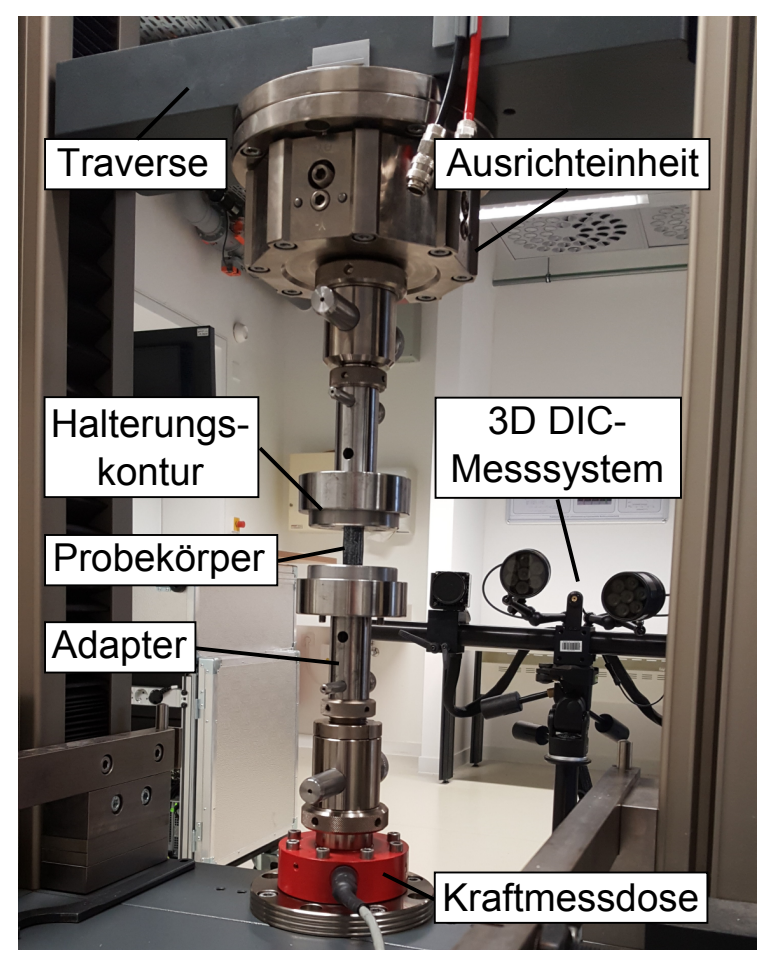

Abb. 3.44 - Versuchsaufbau des Probekörper-Setups mit Messsystem

Adapter (2), die jeweils einen Halterungsring (3), die Halterungskonturen (4) mit Schrauben (5) und den Probekörper umfassen. Die Adapter besitzen eine Bohrung mit Spielpassung (6) und können jeweils über einen Stift (7) und eine Kontermutter (8) mit der Prüfmaschine verbunden werden. Das Probekörper-Setup wird in Reihe mit einer Kraftmessdose (Nennlast: $20 \mathrm{kN}$ ) und einer Ausrichteinheit verbunden. Insbesondere für hochpräzise Druckmessungen spielt die Ausrichteinheit eine entscheidende Rolle. Da die verschiedenen in Reihe geschalteten Anbauteile keine zufriedenstellende Koaxialität besitzen, wird eine Ausrichteinheit zur Korrektur von Exzentrizitäten und Winkelabweichungen benötigt. Die durchgeführte Winkel- und Positionskorrektur kann mit Hilfe von Parallelendmaßen am Probekörper-Setup kontrolliert werden. In der Abbildung 3.46 ist ein schematischer Versuchsaufbau dargestellt, der die Notwendigkeit einer Ausrichteinheit verdeutlicht. Über die 

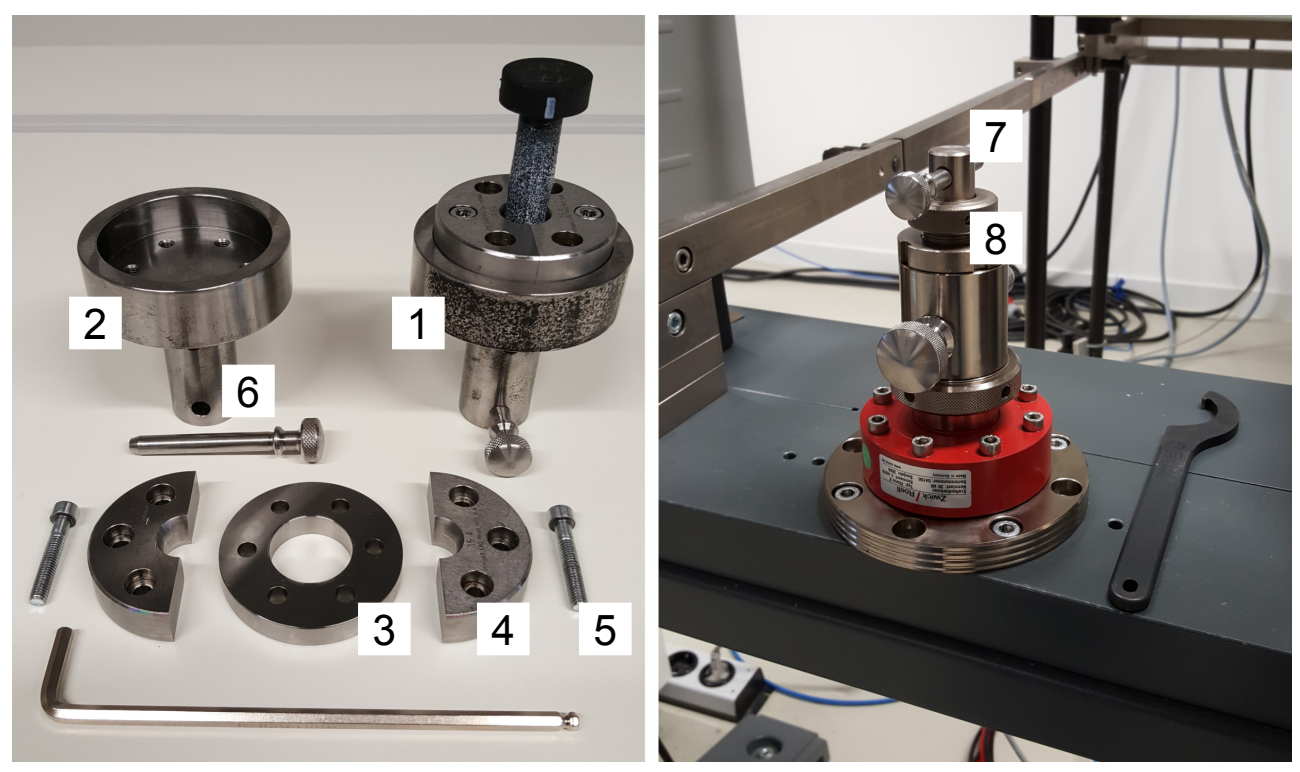

Abb. 3.45 - Zusammenbau des Probekörper-Setups
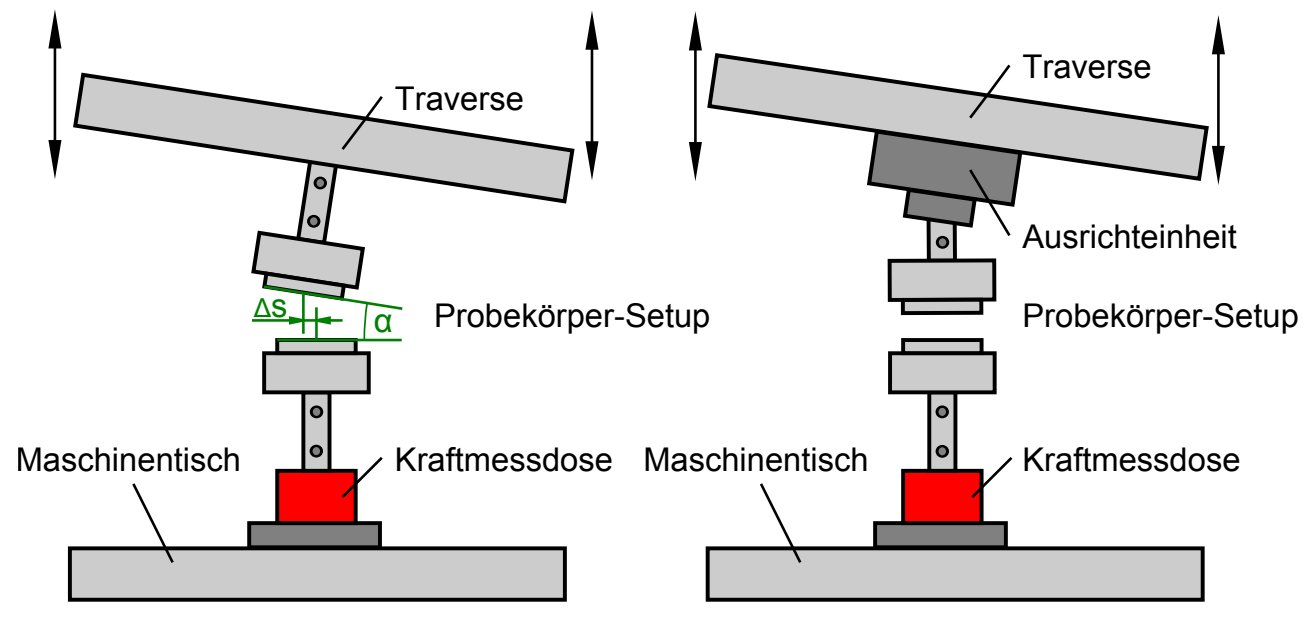

Versuchsaufbau ohne Ausrichteinheit

Versuchsaufbau mit Ausrichteinheit

Abb. 3.46 - Versuchsaufbau mit und ohne Ausrichteinheit

Traverse können weg- oder kraftgesteuerte Versuche gefahren werden. Die Standarduntersuchungen werden mit einer elektromechanischen Zug-/Druckprüfmaschine von der Firma Zwick/Roell durchgeführt. Für dynamische Untersuchungen steht eine servo-hydraulische Prüfmaschine von der Firma Instron zur Verfügung. Auf die Prüfmaschinensteuerung und deren Erweiterung soll im nächsten Abschnitt gesondert Bezug genommen werden. Die Verschiebungsmessung am Probekörper erfolgt, wenn nicht anders angegeben, optisch mit einem 3D Digital Image Correlation-Messsystem (DIC-Messsystem) von der Firma GOM (Aramis 3D 4M-System). Auf die Besonderheiten und Feineinstellungen des hochpräzisen 3D Messsystems, wird an entsprechender Stelle eingegangen. Das Prinzip der DIC-Messung kann dem Buch von Sutton u. a. (2009) entnommen werden. 


\subsubsection{Erweiterte Prüfmaschinensteuerung}

Standardmäßig besitzen moderne Prüfmaschinen (z.B. Zwick/Roell) einen grafischen Ablaufeditor (ein Teil von testXpert II) mit dessen Hilfe Prüfabläufe in Blöcken programmiert werden können. Diese Herangehensweise bietet für Standarduntersuchungen und einfache Prüfsequenzen eine komfortable Handhabbarkeit. Der große Nachteil besteht aber darin, dass keine beliebigen freien Funktionsverläufe generiert werden können. Die standardmäßig zur Verfügung stehenden grafischen Blöcke sind für solche Anwendungsfälle nicht geeignet. Es lassen sich beispielsweise keine kontinuierlichen Übergänge zwischen verschiedenen Sinusbausteinen programmieren. Diese Übergänge werden automatisch mit Haltezeiten und Anfahrrampen verbunden (vgl. Abbildung 3.47 oben). Gerade im Bereich der Ma-

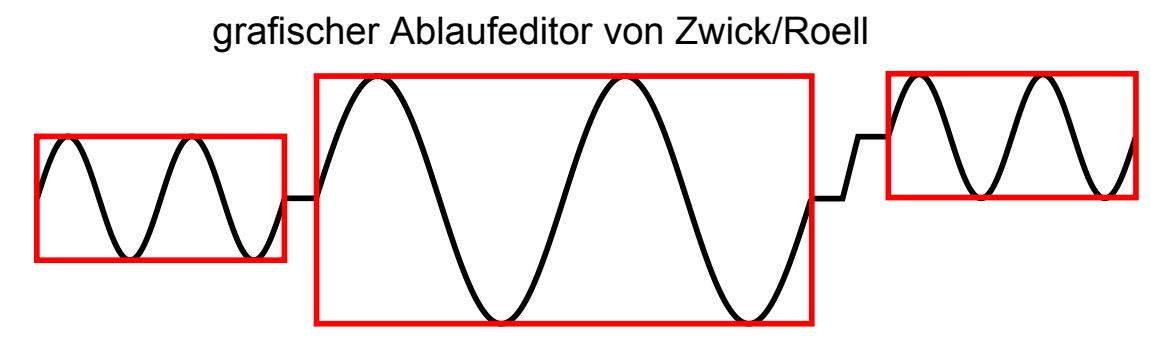

1. Sinusbaustein

2. Sinusbaustein

3. Sinusbaustein

Verwendung der externen Sollwertsteuerung

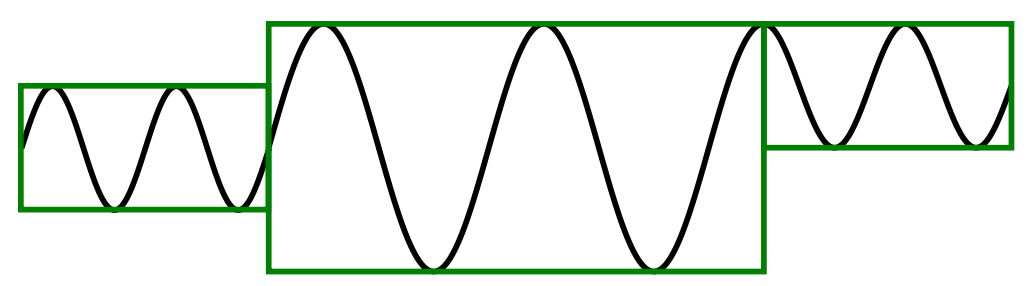

Abb. 3.47 - Gegenüberstellung: grafischer Ablaufeditor von Zwick/Roell und Erweiterung mit der externen Sollwertsteuerung. Die Abbildung ist in leicht modifizierter Form Kanzenbach u. a. (2016a) entnommen. Wiederverwendung mit Genehmigung. (C) 2016 Elsevier Ltd.

terialcharakterisierung, wo kontinuierliche Übergänge sehr wichtig sind, stellt dies einen großen Nachteil dar. Das Ziel besteht in der Erweiterung bzw. Modifizierung der standardmäßigen Steuerung zur Modellierung freier Funktionsverläufe. Mit Hilfe einer externen Sollwertsteuerung soll dieses Ziel realisiert werden. Die Grundidee besteht darin, der Prüfmaschine extern ein vordefiniertes Steuersignal vorzugeben, was dann von der Traverse verfahren wird. In der Abbildung 3.48 ist das Blockdiagramm zur Realisierung der externen Sollwertsteuerung dargestellt. Zu Beginn wird mit Hilfe einer Mathematik-Software (z.B. Matlab) der gewünschte Funktionsverlauf erstellt. Dieser Funktionsverlauf wird im Anschluss in LabVIEW verarbeitet und in ein analoges Ausgangssignal umgewandelt. Dieses Signal, welches in einem Bereich von $\pm 10 \mathrm{~V}$ aufgelöst ist, steuert dann die Traverse. Die Implementierung wurde so realisiert, dass die Standardsoftware von Zwick/Roell weiterhin genutzt werden kann, es aber jederzeit möglich ist, über einen neuen Software- 


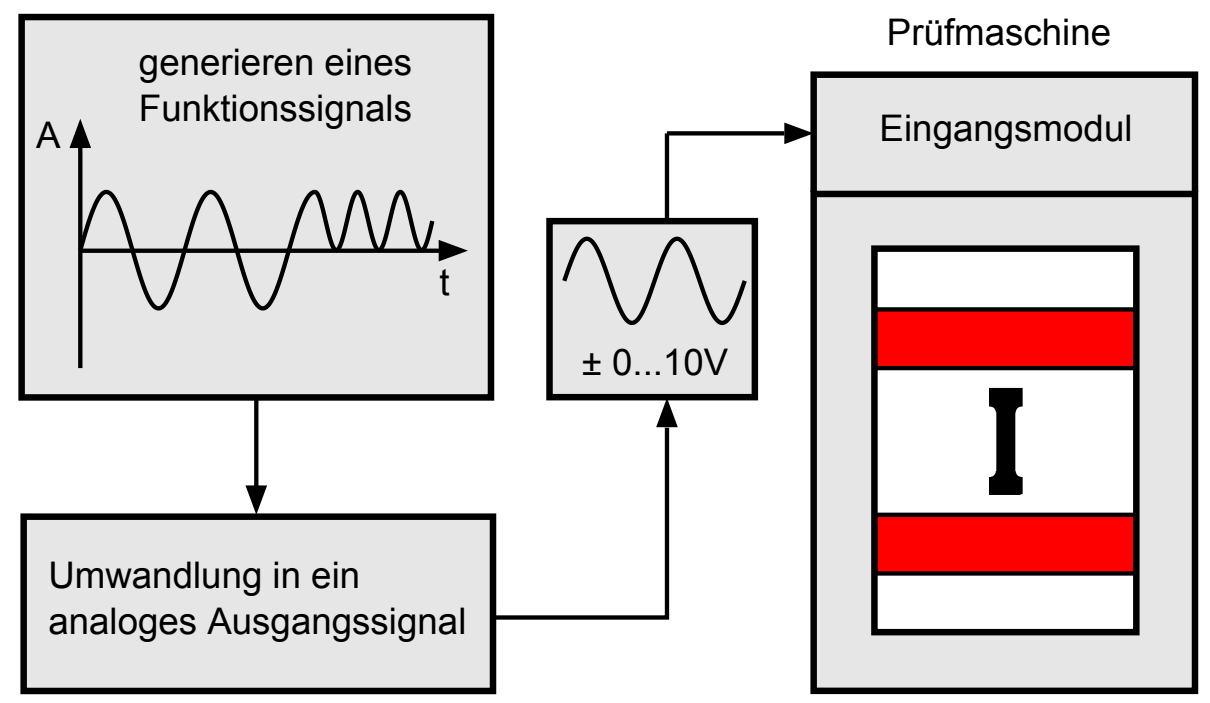

Abb. 3.48 - Blockdiagramm zur Realisierung der externen Sollwertsteuerung. Die Abbildung ist in leicht modifizierter Form Kanzenbach u. a. (2016a) entnommen. Wiederverwendung mit Genehmigung. () 2016 Elsevier Ltd.

baustein das gewünschte Funktionssignal zu verfahren (vgl. Abbildung 3.47 unten). Durch diese Erweiterung entsteht eine Modellierungsfreiheit, die im Bereich der Materialcharakterisierung ganz neue Untersuchungsmöglichkeiten eröffnet. Mit der erweiterten Prüfmaschinensteuerung wurde ein Werkzeug geschaffen, mit dem nun beliebige Amplituden-, Frequenz-, und/oder Offsetwechsel erstellt und verfahren werden können. Damit erlaubt die Prüfmaschine neben der Steuerung vorgegebener Funktionsmodule auch die direkte Vorgabe der Traversenbewegung durch freie analoge Eingangssignale. Dadurch kann die Bewegung komplett frei gesteuert werden und ist nur noch durch „harte“ interne Größen wie Trägheits- und Antriebskräfte begrenzt. Sämtliche nur denkbare Funktionsverläufe im Rahmen der physikalischen Möglichkeiten der Maschine lassen sich somit modellieren und abfahren. In der Veröffentlichung von Kanzenbach u.a. (2016a) sind weitere Einzelheiten und ein Anwendungsbeispiel zur externen Sollwertsteuerung vorgestellt. In den nachfolgenden Abschnitten kann nun auf die erweiterte Prüfmaschinensteuerung zur phänomenologischen Untersuchung von technischen Elastomeren zurückgegriffen werden.

\subsubsection{Voruntersuchungen und Qualitätsprüfungen}

Bevor mit der phänomenologischen Untersuchung technischer Elastomere begonnen werden kann, soll das Probekörper-Setup auf seine Funktionstauglichkeit hin getestet werden. Weiterhin werden Standards für die Messprozedur festgelegt, die es später erlauben, gezielt messen und reproduzierbar Eigenschaften von Elastomeren untersuchen zu können. Die Messergebnisse sollen im Anschluss den Simulationsdaten gegenübergestellt und die Vorhersagegenauigkeit abgeschätzt werden. Durch diese systematische Vorgehensweise können Fehlerquellen eliminiert und das Probekörper-Setup für die industrielle Anwendung nutzbar gemacht werden. Folgende Kriterien/Aspekte sollen zunächst näher untersucht 
werden:

- Homogenität im Messbereich bei großen Stauchungen

- Einfluss von Imperfektionen im Druckversuch

- Einfluss der Reibung zwischen Probekörper und Halterungskontur

- Validierung der verschiedenen Halterungskonturen

Um diese Kriterien genau erfassen und charakterisieren zu können, bedarf es einer geeigneten Messstrategie. Dabei lassen sich die verschiedenen Messsysteme zunächst in berührende und nicht berührende Verfahren einteilen. Vertreter für berührende Messsysteme wären der Clip Gage oder der MultiXtens (Zwick/Roell-Messsystem, welches in das PrüfmaschinenSetup integriert wird). Beide Systeme funktionieren nach dem Prinzip eines Längenänderungsaufnehmers (Extensometer). Für die nicht berührenden Systeme bietet sich z.B. ein Laserextensometer oder die Grauwertkorrelation an (im Folgenden abgekürzt mit DIC). Da die berührenden Systeme gerade für große Stauchungen aufgrund des sich stetig verringernden Messbereiches und der Verstärkung von Imperfektionen ungeeignet sind, soll weitestgehend auf diese Systeme verzichtet werden. Es sei an dieser Stelle aber darauf hingewiesen, dass für reine Zugversuche und Druckversuche bis maximal $20 \%$ Stauchung ein MultiXtens verwendet werden kann. Im direkten Vergleich zwischen Laserextensometer und einem DIC-System besitzt letzteres den großen Vorteil, dass es als Feldmessverfahren einsetzbar ist. Dadurch kann ein großer Messbereich des Probekörpers erfasst und ausgewertet werden. Für die hier durchgeführten Messungen mittels DIC (wenn nicht anders angegeben) erfolgt die axiale Dehnungsberechnung über einen Extensometer: $\varepsilon=\Delta l / l_{\mathrm{M}}$ mit $l_{\mathrm{M}}=20 \mathrm{~mm}$ (vgl. Abbildung 1.1). In Abbildung 3.49 sind diesbezüglich zwei beschich-

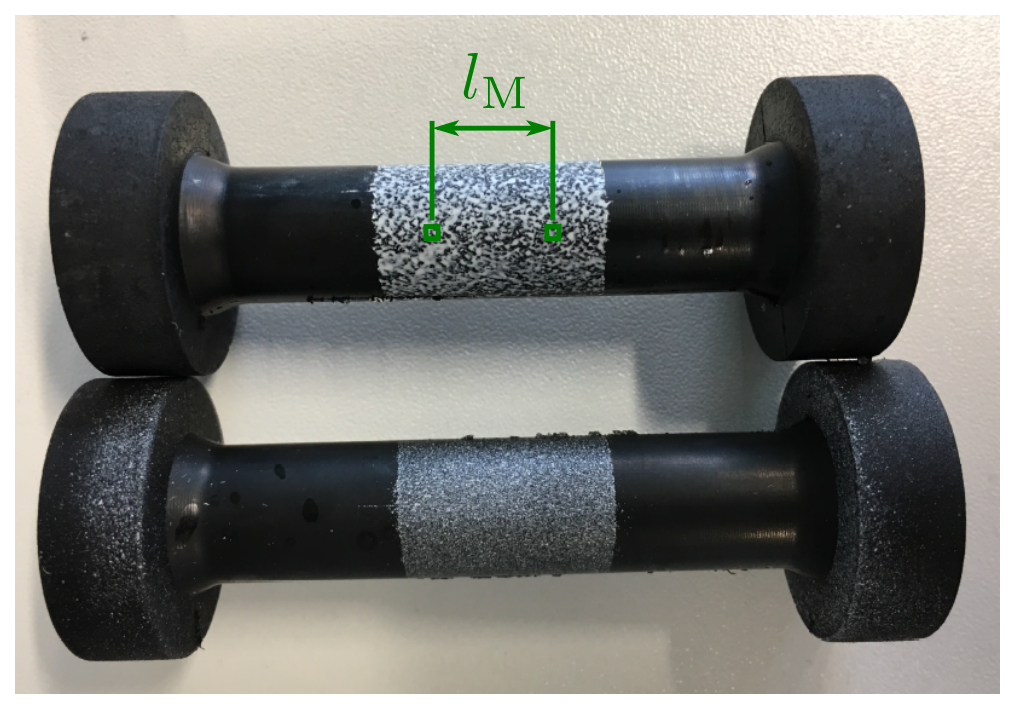

Abb. 3.49 - Hantelprobekörper (gefüllter EPDM) mit unterschiedlicher Beschichtung in Vorbereitung für die Messung mittels DIC (Abbildung in Anlehnung an die Arbeit von Oelsch (2017) durchgeführt an der Professur Festkörpermechanik, TU Chemnitz)

tete Hantelprobekörper dargestellt, die für die DIC-Messung genutzt werden können. Die 
unterschiedliche Beschichtung, die im Rahmen der Arbeit von Oelsch (2017) optimiert wurde, ist auf das verwendete Messvolumen des Aramis-Systems zurückzuführen. Je nach gewähltem Kalibrierobjekt stehen unterschiedliche Messvolumina zur Verfügung, die auf verschieden feine Muster angewiesen sind. Es sei an dieser Stelle mit erwähnt, dass besonders für große Stauchungen eine saubere Präparation der Oberfläche sehr wichtig ist, damit sich bei der Messung keine Beschichtung ablöst und dadurch Facetten ausfallen. Neben der Präparation ist aber auch besonderes Augenmerk auf den spannungsfreien Einbau des Probekörpers in die Prüfmaschine zu legen. Hierbei empfiehlt es sich, die Referenzlänge nach der Montage des Probekörper-Setups zu bestimmen und beim Einbau einzustellen.

Bevor aber die Messung großer Stauchungen erfolgt, soll zunächst ein Stauchversuch ohne Halterungskonturen durchgeführt werden. Dadurch kann zum einen überprüft werden, wie gut der kritische Knickpunkt mit der Vorhersage aus der Simulation übereinstimmt. Zum anderen wird dadurch in sehr praktischer Art und Weise deutlich, wie wichtig die entwickelten Halterungskonturen zur Realisierung hochpräziser Stauchungen sind. In der Abbildung 3.50 ist ein Stauchversuch ohne Halterungskonturen dargestellt. Der Hantelkopf (oben und unten) wird dabei jeweils durch einen Halterungsring geführt (vgl. Euler-IVKnickung). Aus der Abbildung wird deutlich, dass der Knickpunkt bei ca. 22 \% Stauchung

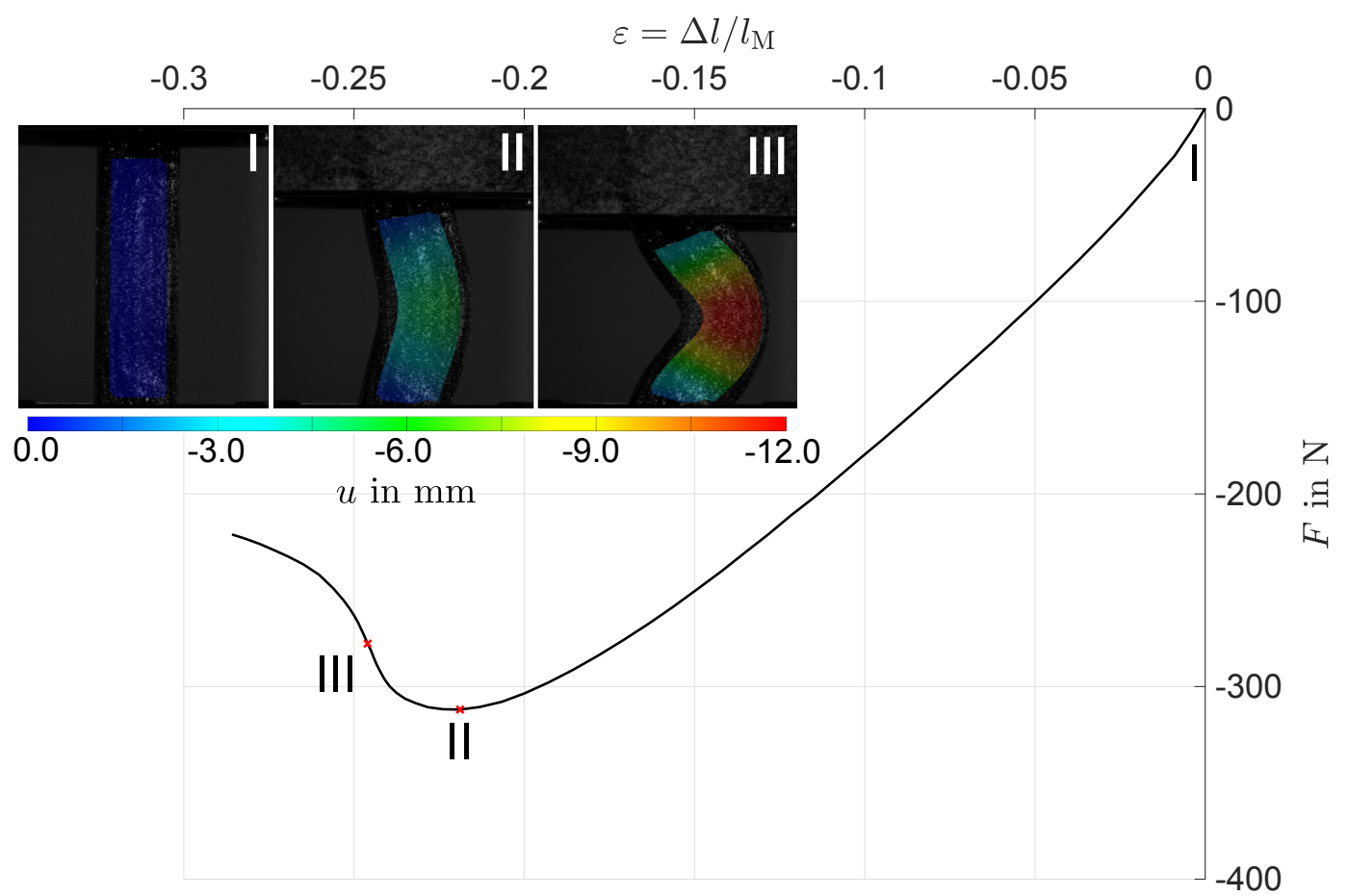

Abb. 3.50 - Stauchversuch ohne Halterungskonturen (Messung für gefüllten EPDM), Messsystem DIC. Quelle der Gesamtabbildung: Kanzenbach u. a. (2019a). Wiederverwendung mit Genehmigung. () 2019 Elsevier Ltd.

liegt. Dieser Wert stimmt sehr gut mit den Ergebnissen aus Abbildung 3.5 und Abbildung 3.8 überein. Damit kann der berechnete Instabilitätspunkt mittels vorgespannter Modal- 
analyse bestätigt werden. Um den Knickprozess weiterhin gut nachvollziehen zu können, sind in der Abbildung 3.50 verschiedene Druckstufen (I bis III) dargestellt. Anhand des Farbverlaufes (Verschiebung $u$ in horizontaler Richtung) wird deutlich, wie sich die erste Knickform symmetrisch ausbildet. Es sei an dieser Stelle mit erwähnt, dass die Knickung etwa senkrecht zur Blickrichtung der Kameras erfolgt.

\section{Homogenität im Messbereich bei großen Stauchungen}

Im Folgenden wird ein Druckversuch mit Halterungskonturen zur Untersuchung der Homogenität im Messbereich bei großen Stauchungen durchgeführt. In Abbildung 3.51 ist ein Druckversuch bei einer Stauchung von 50 \% dargestellt (links Darstellung der Referenzkonfiguration und rechts Darstellung der aktuellen Deformation, vgl. auch Kanzenbach u. a. 2018). Zunächst einmal kann festgehalten werden, dass der Probekörper gleichmäßig ge-

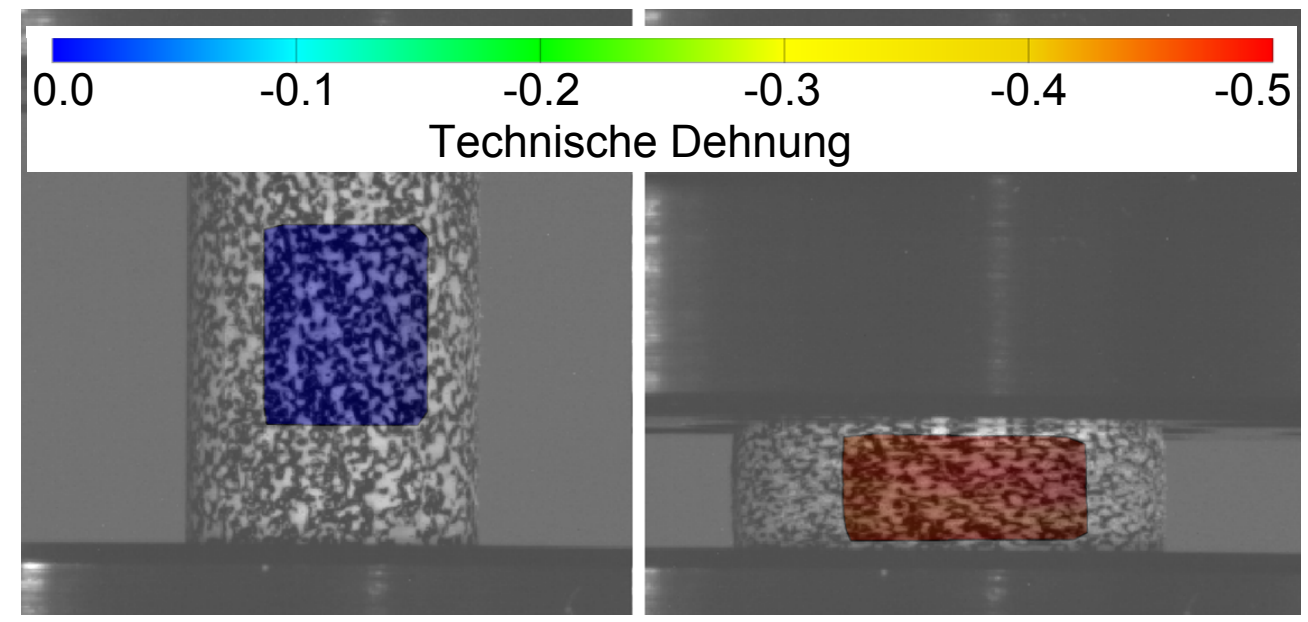

Abb. 3.51 - Stauchversuch des Hantelprobekörpers (gefüllter EPDM) mit Halterungskonturen. Die Abbildung ist in leicht modifizierter Form Kanzenbach u.a. (2018) entnommen. Wiederverwendung mit Genehmigung. () 2018 Elsevier Ltd.

staucht wird und damit die Funktionstauglichkeit des Setups gewährleistet ist. Für diesen Funktionstest wurde die sichere Halterungskontur verwendet. Anhand des Dehnungsfeldes wird deutlich, dass der Probekörper nahezu homogen deformiert wurde. Die Dehnung wird lokal mittels der ausgewerteten Facetten berechnet und dargestellt. Für eine globale Dehnungsauswertung wird das Verschiebungsfeld mit einem optischen Extensometer ausgewertet. Aus Abbildung 3.51 wird weiterhin deutlich, dass der deformierte Probekörper eine leicht tonnenförmige Außenkontur aufzeigt. Diese Kontur stimmt mit Simulationsdaten einer nahezu homogenen Stauchung sehr gut überein. Ein Ausbeulen oder gar Knicken des Probekörpers (vgl. Abbildung 3.50 ist dabei nicht zu erkennen.

\section{Einfluss von Imperfektionen im Druckversuch}

Zur Überprüfung der Knicksicherheit wird als nächstes der Einfluss von Imperfektionen im Stauchprozess untersucht. Dazu wird über den ausgewählten Messbereich des Probe- 
körpers (Zylindersegment) eine Zylinderextrapolation durchgeführt und der Mittelpunktversatz berechnet. Es wird die Annahme zugrunde gelegt, dass der Probekörper seinen Kreisquerschnitt näherungsweise beibehält. Bei einer idealen Stauchung weitet sich der Probekörper in alle Richtungen gleichmäßig aus, was zu einem Mittelpunktversatz von Null führt. In der Abbildung 3.52 ist der Mittelpunktversatz von dem eben gezeigten Druckversuch (Abbildung 3.51) dargestellt. Das Polardiagramm gibt dabei die Richtung

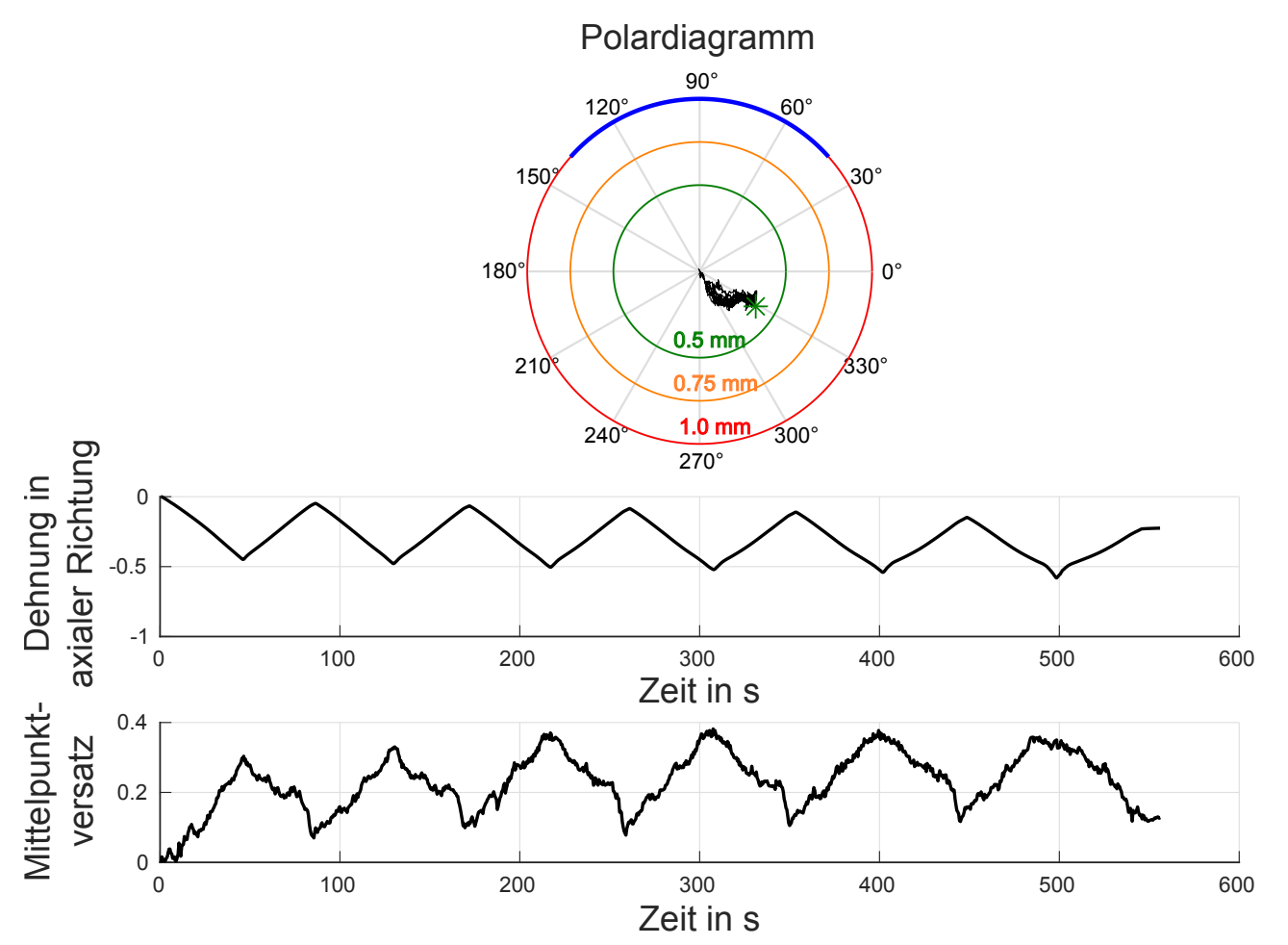

Abb. 3.52 - Darstellung des Mittelpunktversatzes des Hantelprobekörpers (Messung für gefüllten EPDM)

und den Betrag des Auswanderns des Mittelpunktes an. Der blau markierte Sektor kennzeichnet dabei das Blickfeld des 3D DIC-Messsystems. Weiterhin ist in der Abbildung 3.52 die technische Dehnung in axialer Richtung und der Mittelpunktversatz über der Zeit dargestellt. Der größte Mittelpunktversatz beträgt bei der hier verwendeten Halterungskontur $u_{\mathrm{M}} \approx 0.4 \mathrm{~mm}$. Verglichen mit dem Referenzradius führt dies zu einem sehr geringen Instabilitätsmaß $\kappa$. Die Ermittlung des Mittelpunktversatzes stellt somit ein wichtiges Qualitätsmaß für den Stauchprozess dar. Folglich kann festgehalten werden, dass das Probekörper-Setup große Stauchungen ermöglicht und sich mittels der hier verwendeten Halterungskontur präzise deformieren lässt. Der sehr geringe Mittelpunktversatz der Probe unterstreicht zudem die hohe Qualität des gesamten Versuchsaufbaus und der numerischen Vorentwicklung.

\section{Einfluss der Reibung zwischen Probekörper und Halterungskontur}

Für dynamische Untersuchungen, wie beispielsweise die Bestimmung des Speicher- und Verlustmoduls oder die dynamischen Verhärtung, ist es von großem Interesse zu wissen, 
ob zwischen dem Probekörper und der Halterungskontur ein Haftgleiteffekt (Stick-SlipEffekt) auftreten kann. Dieser Effekt würde bei einer dynamischen Belastung zu einer selbsterregten Reibschwingung führen, die das gemessene Materialverhalten verfälschen würde. Im Folgenden werden an einer servo-hydraulischen Prüfmaschine entsprechende Untersuchungen durchgeführt, um den Einfluss unterschiedlicher Reibpaarungen zu charakterisieren. In der Abbildung 3.53 ist der Versuchsaufbau des Probekörper-Setups an einer servo-hydraulischen Prüfmaschine von der Firma INSTRON dargestellt. Besonderes
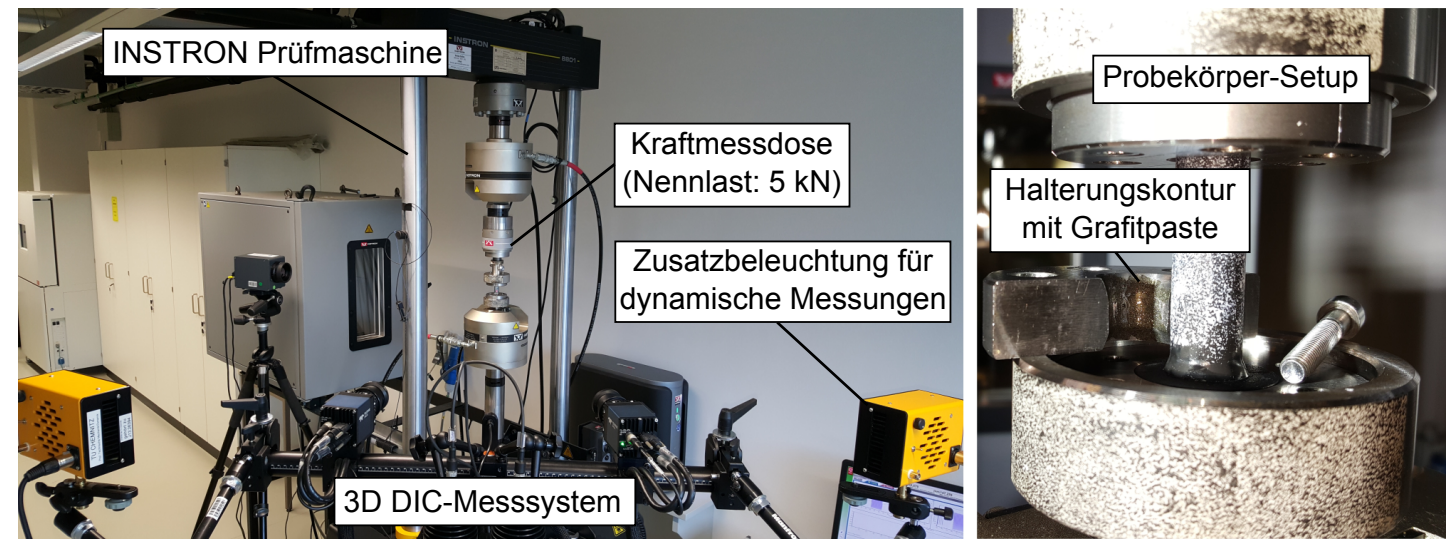

Abb. 3.53 - Versuchsaufbau zur Untersuchung des Reibeinflusses

Augenmerk soll zunächst auf die Messmethodik bei höheren Prüffrequenzen gelegt werden. Im Gegensatz zu quasi-statischen Versuchen muss bei dynamischen Versuchen mit einer Zusatzbeleuchtung (Typ MultiLED PT von der Firma GS Vitec) gearbeitet werden, um die Belichtungszeit des 3D DIC-Messsystems zu verringern. Soll beispielsweise bei einer Belastungsfrequenz von $10 \mathrm{~Hz}$ eine Sinusschwingung mit 20 Messpunkten abgetastet werden, so wird eine Messfrequenz von $200 \mathrm{~Hz}$ benötigt. Bei voller Auflösung des DIC-Systems ist eine maximale Messfrequenz von $168 \mathrm{~Hz}$ möglich. Durch eine weitere Reduzierung des Bildbereiches auf $1 / 2$ oder $1 / 4$ kann die benötigte Messfrequenz erreicht werden. Mit Hilfe der Zusatzbeleuchtung lässt sich dann die benötigte kurze Belichtungszeit realisieren. Die Versuche sollen im Folgenden so gestaffelt sein, dass zuerst zwei Versuche ohne Grafitpaste (Schmiermittel) durchgeführt werden, um die Wiederholgenauigkeit der Messungen zu erfassen. Im Anschluss daran erfolgt ein Wiederholungsversuch mit Umbau des Probekörper-Setups, um den Einfluss der Montage zu ermitteln. Abschließend erfolgen zwei Versuche, bei denen die Halterungskonturen mit Grafitpaste präpariert sind. In Abbildung 3.53 (rechts) ist der Umbau und die präparierte Halterungskontur mit Grafitpaste dargestellt. Die Ergebnisse der durchgeführten Reibuntersuchungen sind in der Abbildung 3.54 zusammengestellt. Hierbei wurde der dynamische Modul über der Frequenz aufgetragen, um Unterschiede besser veranschaulichen zu können. Aus der Abbildung 3.54 wird deutlich, dass die Versuche mit Grafitpaste zu keiner nennenswerten Änderung des absoluten Niveaus und des Anstieges führen. Der Effekt der dynamischen Verhärtung (Materialversteifung über der Frequenz) kann sowohl mit als auch ohne Grafitpaste sehr präzise 


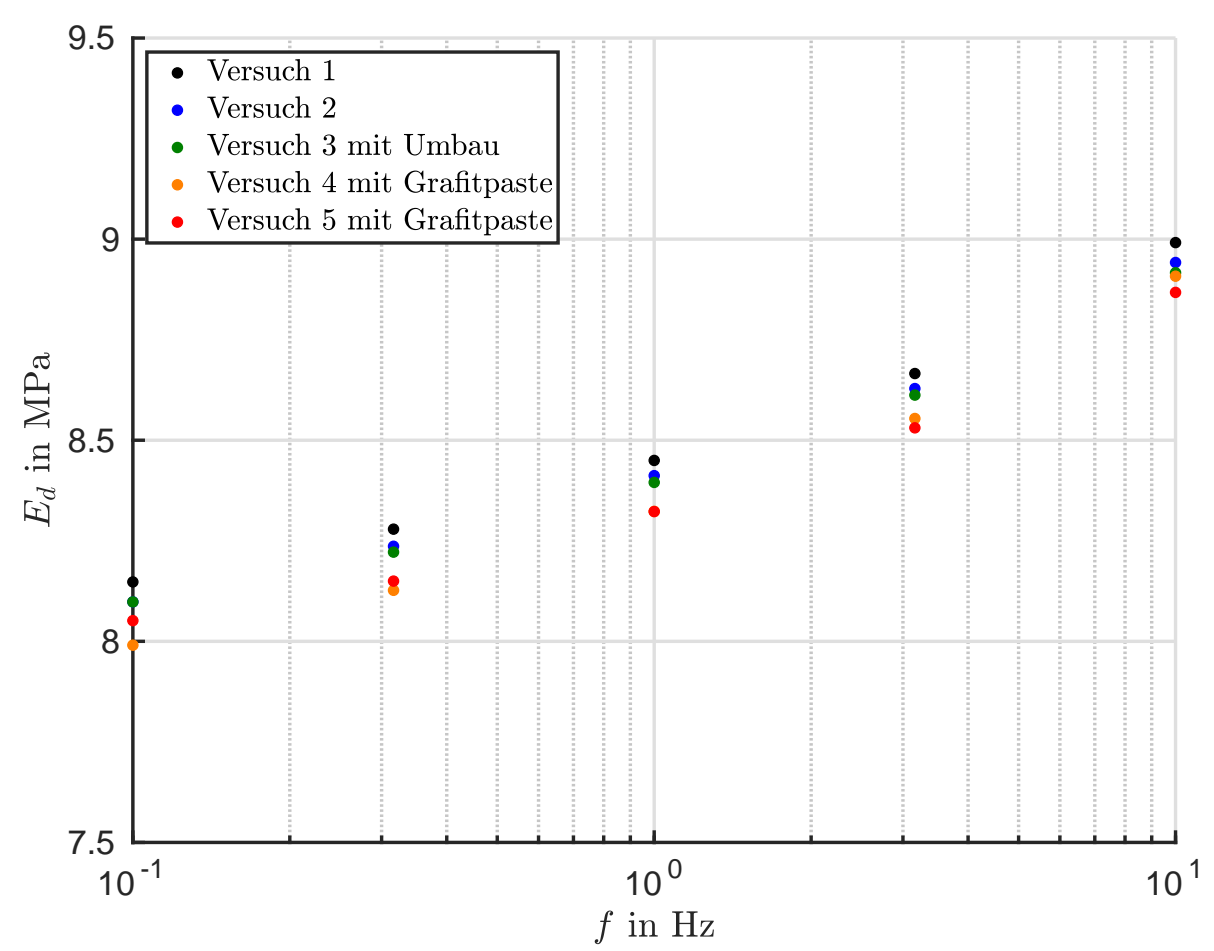

Abb. 3.54 - Darstellung des dynamischen Moduls über der Frequenz zur Untersuchung des Einflusses der Reibung (Messungen für gefüllten EPDM)

erfasst werden. Durch diese Untersuchungen kann sichergestellt werden, dass unterschiedliche Reibpaarungen zwischen Probekörper und Halterungskontur zu keiner Verfälschung der Messergebnisse führen.

\section{Validierung der verschiedenen Halterungskonturen}

Als nächstes werden die Messergebnisse der verschiedenen Halterungskonturen miteinander verglichen und den Simulationsdaten gegenübergestellt. Dazu wird mit jeder Halterungskontur und jeweils mit einem neuen Probekörper (gefüllter EPDM) ein separater Druckversuch bis ca. 50 \% Stauchung durchgeführt. In Abbildung 3.55 sind die Druckversuche für die verschiedenen Halterungen dargestellt. Zunächst soll auf die Halterungskonturen aus der Parameterstudie eingegangen werden. Aus der Abbildung 3.55 wird deutlich, dass die Spannungs-Dehnungskurven bis 30 \% Stauchung zu sehr ähnlichen Ergebnissen führen. Erst bei größeren Stauchungen sind die Einflüsse der verschiedenen Halterungskonturen sichtbar. Hier zeigt sich, dass die sichere Halterung bei gleicher Stauchung größere Spannungswerte aufweist als die kritische Halterung. Legt man die Überlegung zugrunde, dass bei einem erfolgreichen Druckversuch (niedriger Mittelpunktversatz) die SpannungsDehnungskurven der kritischen Halterung am dichtesten an der homogenen Lösung liegen, so zeigt sich, dass die sichere Halterung den stärksten Einfluss auf die Kurven ausübt. Die Standard-Halterung liegt mit ihrem Verlauf zwischen der sicheren und der kritischen Halterungskontur. Diese Reihung passt sehr gut mit den Simulationsergebnissen aus der Abbildung 3.13 zusammen. Die Spannungs-Dehnungskurve, welche aus der optimierten 


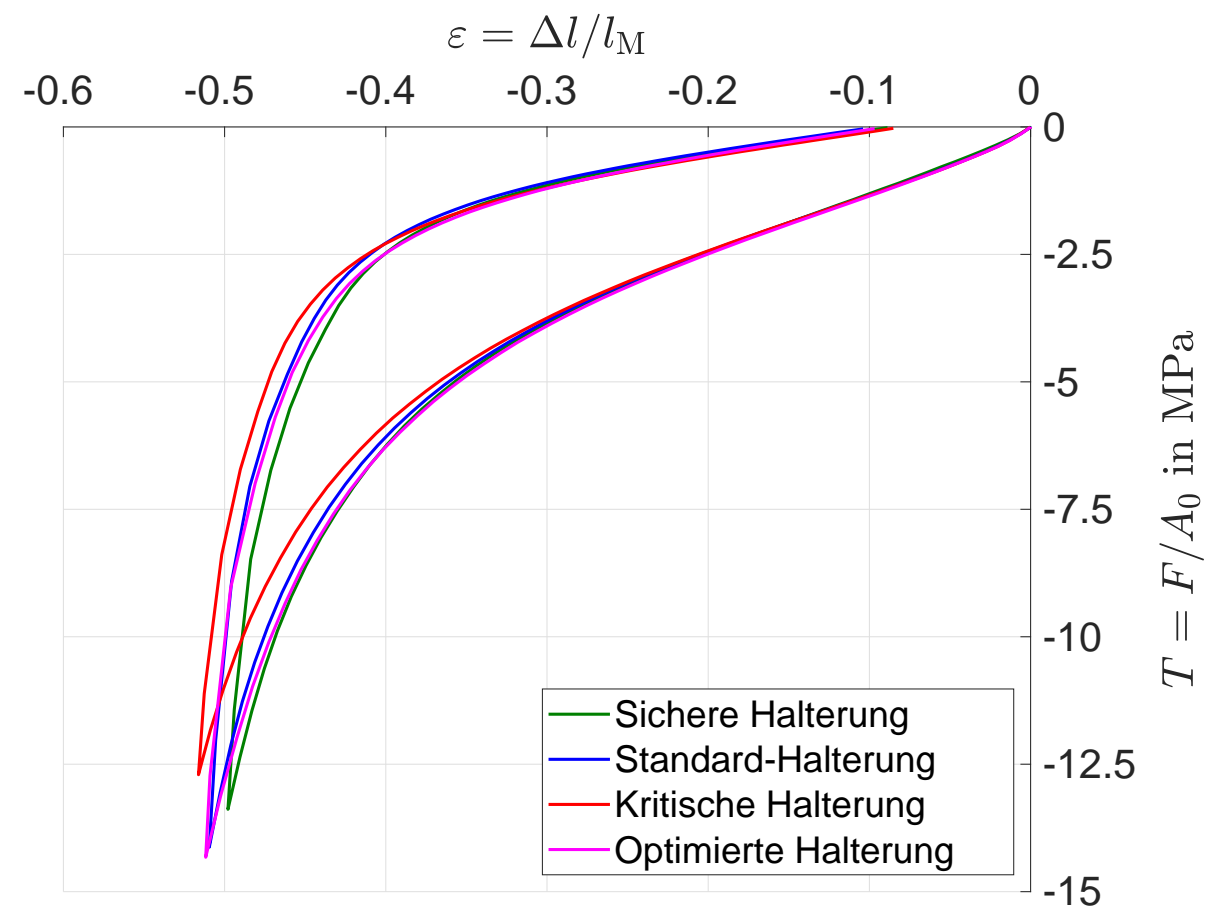

Abb. 3.55 - Vergleich der Halterungskonturen bei einer Stauchung von ca. $50 \%$ (Messungen für gefüllten EPDM), Messsystem DIC

Halterung resultiert, liegt zunächst zwischen dem Verlauf der sicheren und der StandardHalterung. Bei genauerer Betrachtung wird deutlich, dass die optimierte Halterung anfänglich nah an dem Verlauf der sicheren Halterungskontur liegt und sich dann dem Verlauf der Standard-Halterung anpasst. Dieser Trend stimmt ebenfalls gut mit den Simulationsdaten überein. Es bleibt zu prüfen, ob sich neben den verbesserten Eigenschaften in der Homogenität, auch das Stabilitätsverhalten dem der sicheren Halterung angepasst hat. In Abbildung 3.56 sind die Mittelpunktabweichungen der verschiedenen Halterungskonturen gegenübergestellt. Zunächst sollen wieder die Halterungskonturen aus der Parameterstudie diskutiert werden. Aus der unteren Abbildung wird deutlich, dass die kritische Halterung zum größten Mittelpunktversatz führt $\left(u_{\mathrm{M}} \approx 0.6 \mathrm{~mm}\right)$ und die sichere Halterung zum zweitniedrigsten $\left(u_{\mathrm{M}}=0.4 \mathrm{~mm}\right)$. Die Standard-Halterung liegt mit ihren Werten wieder im mittleren Bereich. Diese Reihung stimmt quantitativ gut mit den bisherigen Simulationen überein. Für die optimierte Halterung ergibt sich sogar ein niedrigerer Mittelpunktversatz als für die sichere Halterungskontur. Die als positiv zu wertenden Unterschiede sind aber nur marginal. Entscheidend ist, dass die optimierte Halterung die Vorzüge aus der Standard-Halterung (Homogenität) und der sicheren Halterung (Stabilität) vereinigt. An dieser Stelle soll nochmal auf die Bedeutung der Ausrichteinheit und der ganzen Versuchsmethodik hingewiesen werden, die insgesamt zu sehr guten Messergebnissen führt. Da das hier entwickelte Probekörper-Setup später für die industrielle Anwendung genutzt werden soll, ist ein besonderer Schwerpunkt auf die Knicksicherheit und die damit verbundene Reduzierung des Mittelpunktversatzes gelegt. 


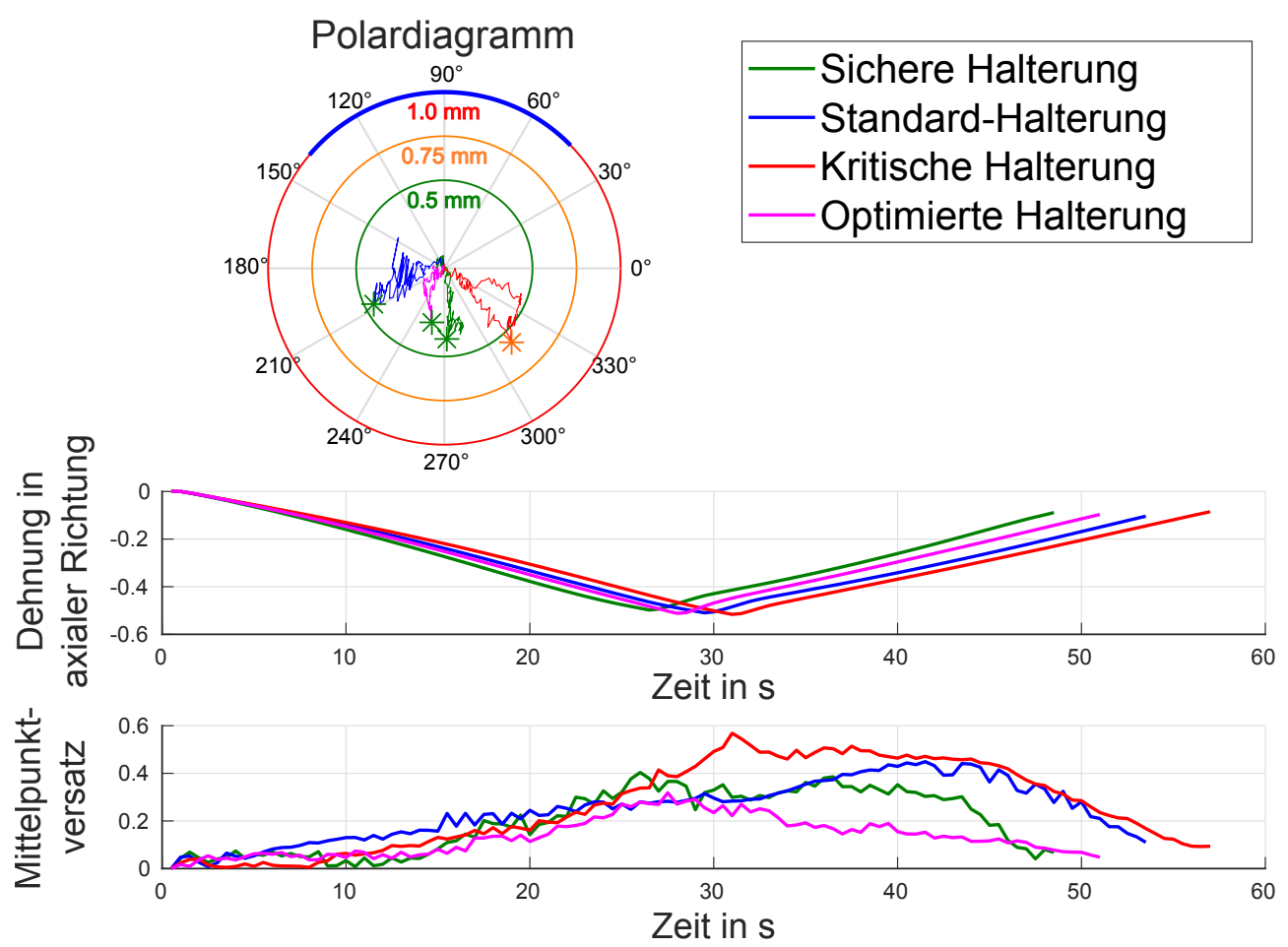

Abb. 3.56 - Darstellung des Mittelpunktversatzes für die verschiedenen Halterungskonturen (Messungen für gefüllten EPDM)

\section{Fazit der Voruntersuchungen und Qualitätsprüfungen:}

Nach Abschluss aller Voruntersuchungen kann festgehalten werden, dass das ProbekörperSetup den gewünschten Anforderungen gerecht wird. Die Homogenität bei großen Stauchungen ist experimentell gewährleistet und mit Hilfe der Messung des Mittelpunktversatzes kann der Einfluss von Imperfektionen überprüft werden. Darüber hinaus zeigt die optimierte Halterungskontur die beste Performance bezüglich Homogenität und Stabilität.

\subsubsection{Phänomenologische Untersuchung technischer Elastomere}

Mit Hilfe des entwickelten Probekörper-Setups sollen im Folgenden eine Reihe phänomenologischer Eigenschaften von Elastomerwerkstoffen untersucht werden. In den vorangegangenen Abschnitten wurde bereits anhand von FE-Simulationen gezeigt, dass sich der Probekörper im Messbereich homogen deformieren lässt. Somit stellt er einen idealen Probekörper für die Untersuchung großer Zug- und Druckbelastungen dar. Darüber hinaus können eine Vielzahl von Prüfabläufen wie kombinierte Zug-/Druckversuche, MultiHystereseversuche, Relaxations- und Kriechversuche gefahren werden. 


\section{Gestufte Zugversuche}

Anhand eines mehrstufigen zyklischen Zugversuches (drei Zyklen pro Laststufe) können die grundlegenden Eigenschaften von Elastomeren beschrieben werden. In der Abbildung 3.57 ist ein vollständiger Zugversuch an gefülltem EPDM dargestellt und in der Abbildung 3.58 einer für gefüllten Naturkautschuk (vgl. dazu auch Kanzenbach u. a. 2016b \& 2018).

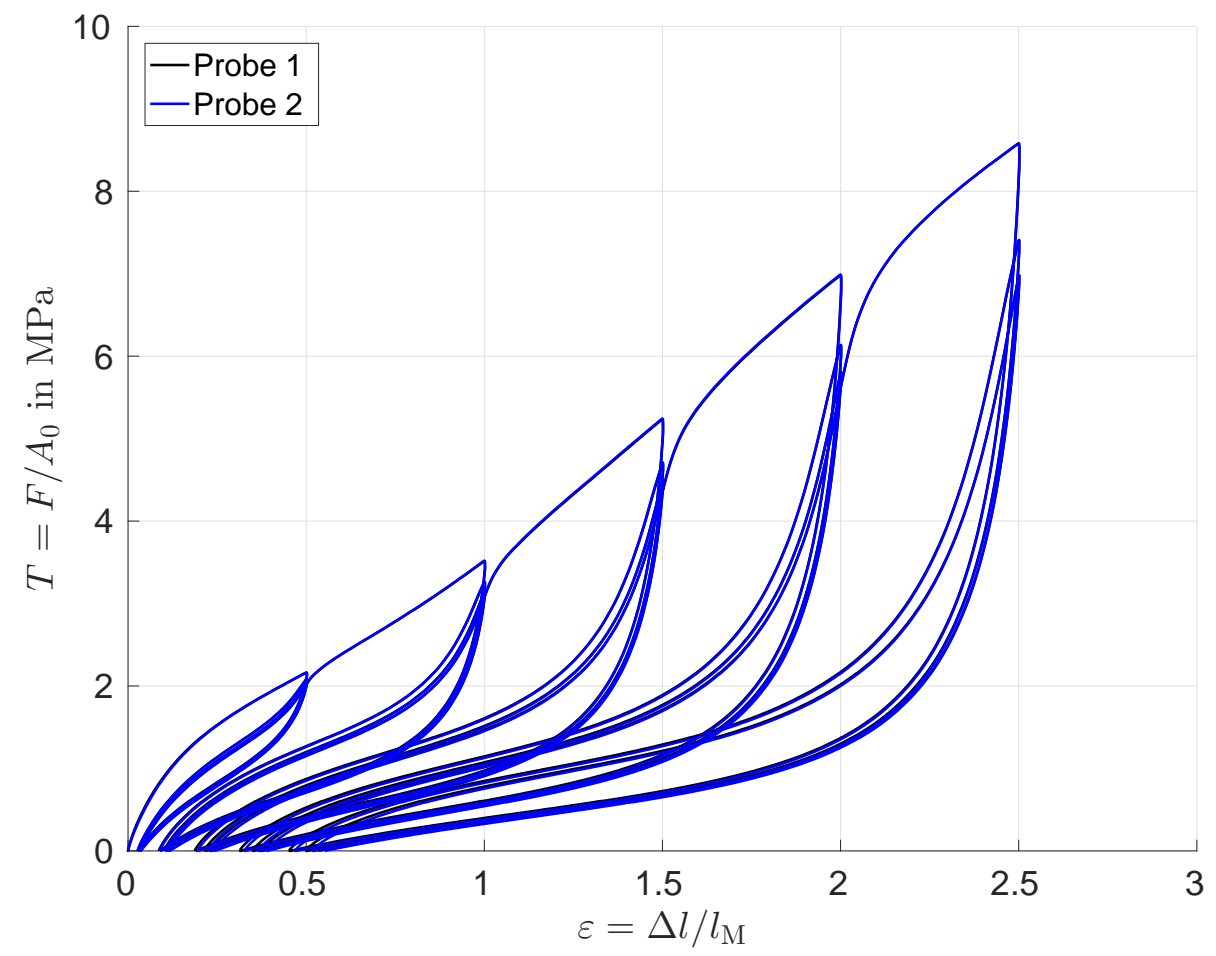

Abb. 3.57 - Mehrstufiger Zugversuch (Messungen für gefüllten EPDM), Messsystem MultiXtens (Die Kurven schwarz und blau fallen zusammen)

Anhand der Abbildungen lassen sich verschiedene Entfestigungs-Effekte detektieren. Zum einen tritt Entfestigung bei wiederholter Deformation, also Lastwiederholung auf, zum anderen lässt sich eine Entfestigung durch Vorreckung erkennen. Beide Entfestigungs-Effekte werden in dieser Arbeit als Aspekte des Mullins-Effekt bezeichnet (vgl. Mullins 1948). Für eine Vertiefung zur Begrifflichkeit und Zuordnung des Mullins-Effekts sei auf die Arbeit von Ihlemann (2003) verwiesen. Charakteristisch für gefüllten EPDM und gefüllten Naturkautschuk sind die großen Hysteresen. Der Inhalt der Hysteresen gibt dabei die dissipierte Energie des jeweiligen Belastungszyklus an. Hieran wird deutlich, dass für eine erstmalige Belastung viel mehr Arbeit benötigt wird. Als letztes soll auf die bleibende Dehnung im Ursprung eingegangen werden. Der mehrstufige Zugversuch wurde dabei so konzipiert, dass nach einer Belastung wieder auf Kraft Null zurückgefahren wird. Hierbei wird erkennbar, dass der Probekörper nicht wieder in seine Ursprungsform zurückgeht, sondern eine neue Referenzlage aufweist. Bei ausreichend langer Ruhezeit ist dieser Effekt der bleibenden Restdehnung teilweise reversibel. Es sei an dieser Stelle schon darauf hingewiesen, dass das neu entwickelte Probekörper-Setup auch für nachfolgende Druckbeanspruchungen 


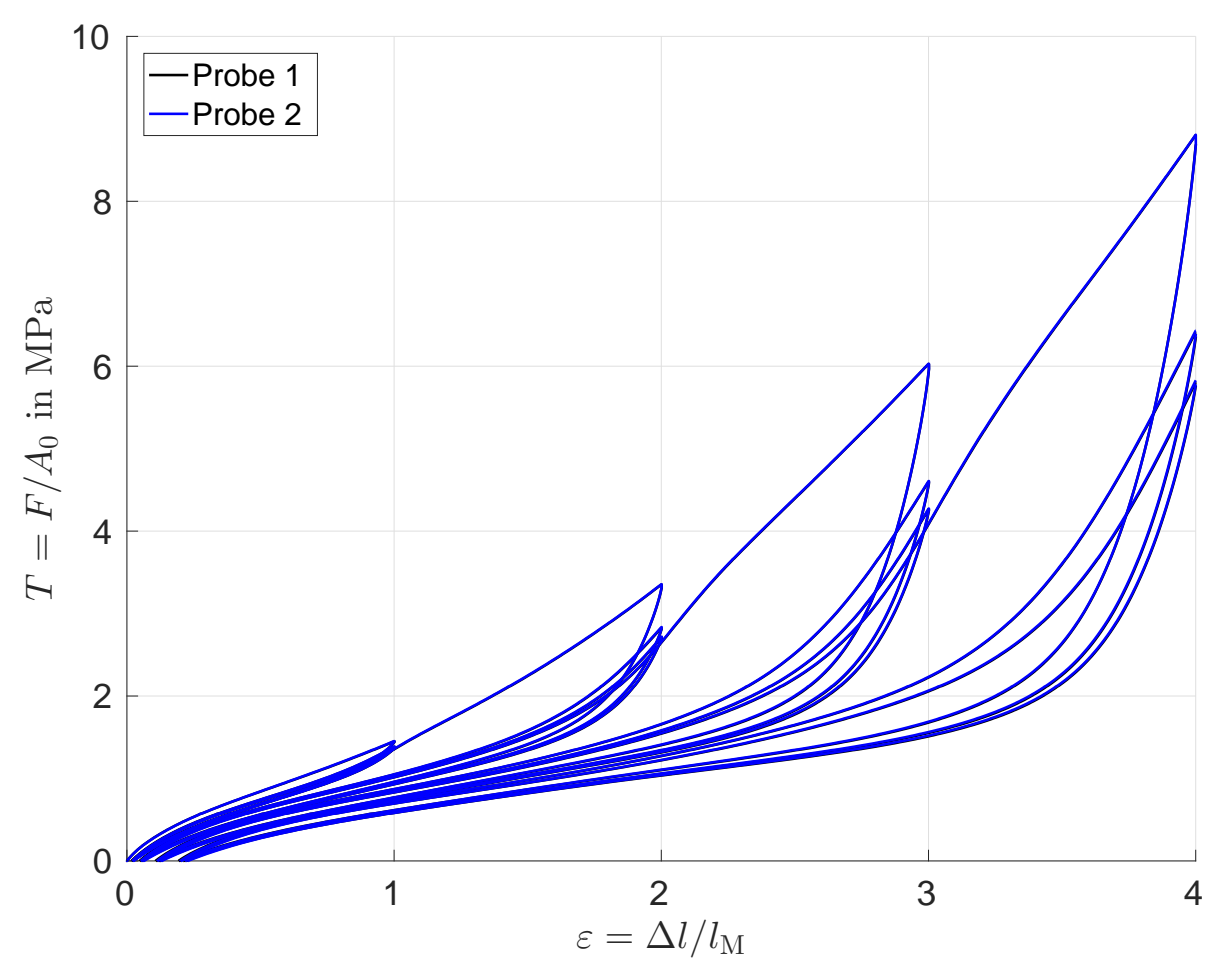

Abb. 3.58 - Mehrstufiger Zugversuch (Messungen für gefüllten NR), Messsystem MultiXtens (Die Kurven schwarz und blau fallen zusammen). Die Abbildung ist in leicht modifizierter Form Kanzenbach u. a. (2016b) entnommen. Wiederverwendung mit Genehmigung. (ㅇ 2016 Wiley-VCH Verlag GmbH \& Co. KGaA, Weinheim

geeignet ist. Der hier verwendete Prüfablauf eignet sich hingegen auch für Versuche mit S2Stäben, welche nicht auf Druck beansprucht werden können. Zusammenfassend lässt sich sagen, dass das Probekörper-Setup einem herkömmlichen S2-Stab in nichts nachsteht und darüber hinaus Druckbeanspruchungen ermöglicht, die im Folgenden untersucht werden sollen.

\section{Gestufte Zug-Druckversuche}

Im Weiteren sollen mit gefüllten EPDM und gefüllten Naturkautschuk gestufte ZugDruckversuche (drei Zyklen pro Laststufe) durchgeführt werden. Die Versuche erfolgen in der Weise, dass zuerst eine reine Druck- gefolgt von einer reinen Zugbeanspruchung realisiert wird (schwarzer Verlauf). Im Anschluss daran wird der Versuchsablauf umgedreht (blauer Verlauf) und die Ergebnisse der Druck-/Zugbeanspruchung werden mit der Zug-/Druckbeanspruchung verglichen. In Abbildung 3.59 und Abbildung 3.60 sind die eben beschriebenen Versuche dargestellt. Anhand der Abbildungen wird zunächst einmal der starke Unterschied der Grundsteifigkeiten der beiden Mischungen von gefüllten EPDM und gefüllten Naturkautschuk deutlich. Es wurden bewusst Mischungen gewählt, die in ihrer Zusammensetzung sehr unterschiedlich sind, um so ein breites Anwendungsspektrum abdecken zu können. Aus der Abbildung 3.59 wird deutlich, dass eine Vorkonditionierung 


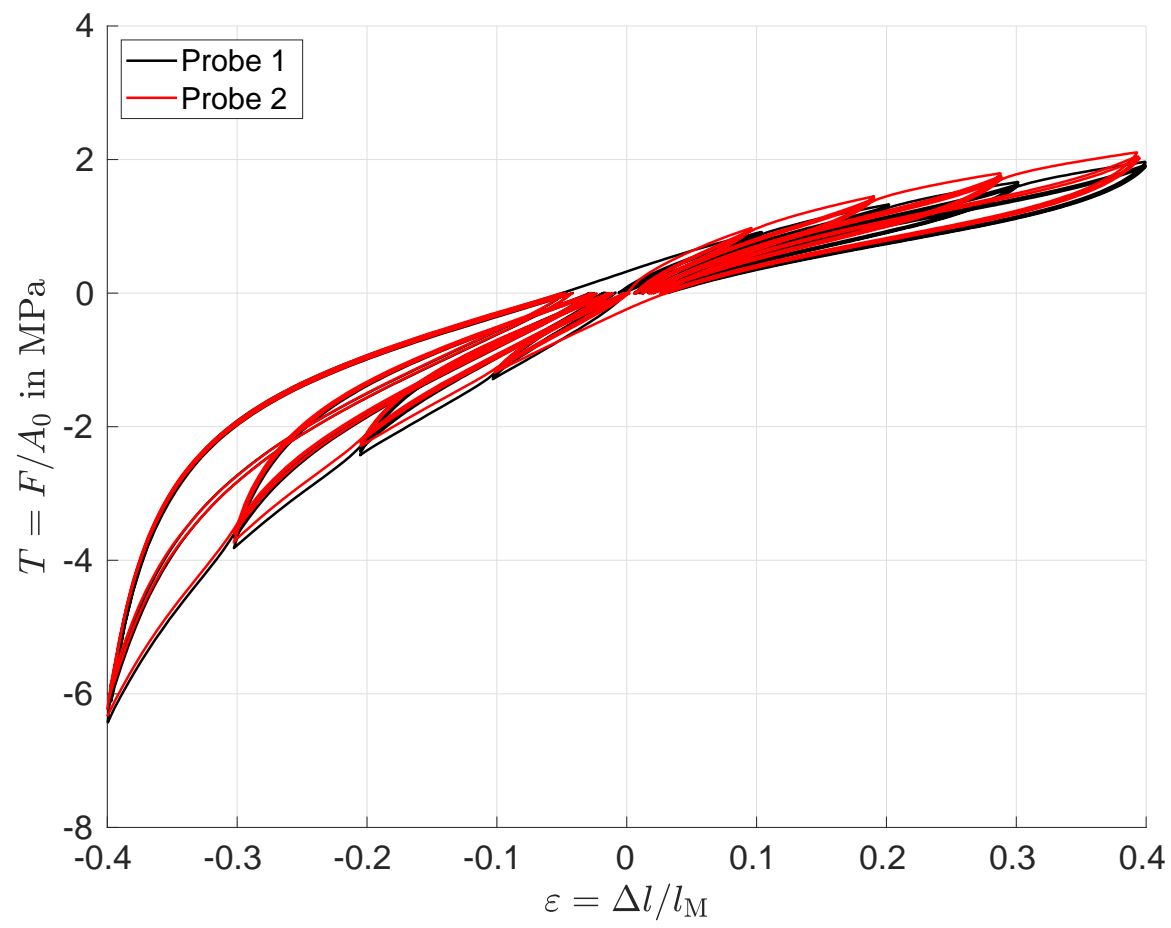

Abb. 3.59 - Vergleich von Druck-Zug (schwarz) und Zug-Druckversuchen (rot) (Messungen für gefüllten EPDM), Messsystem DIC. Die Abbildung ist in leicht modifizierter Form Kanzenbach u. a. (2018) entnommen. Wiederverwendung mit Genehmigung. () 2018 Elsevier Ltd.

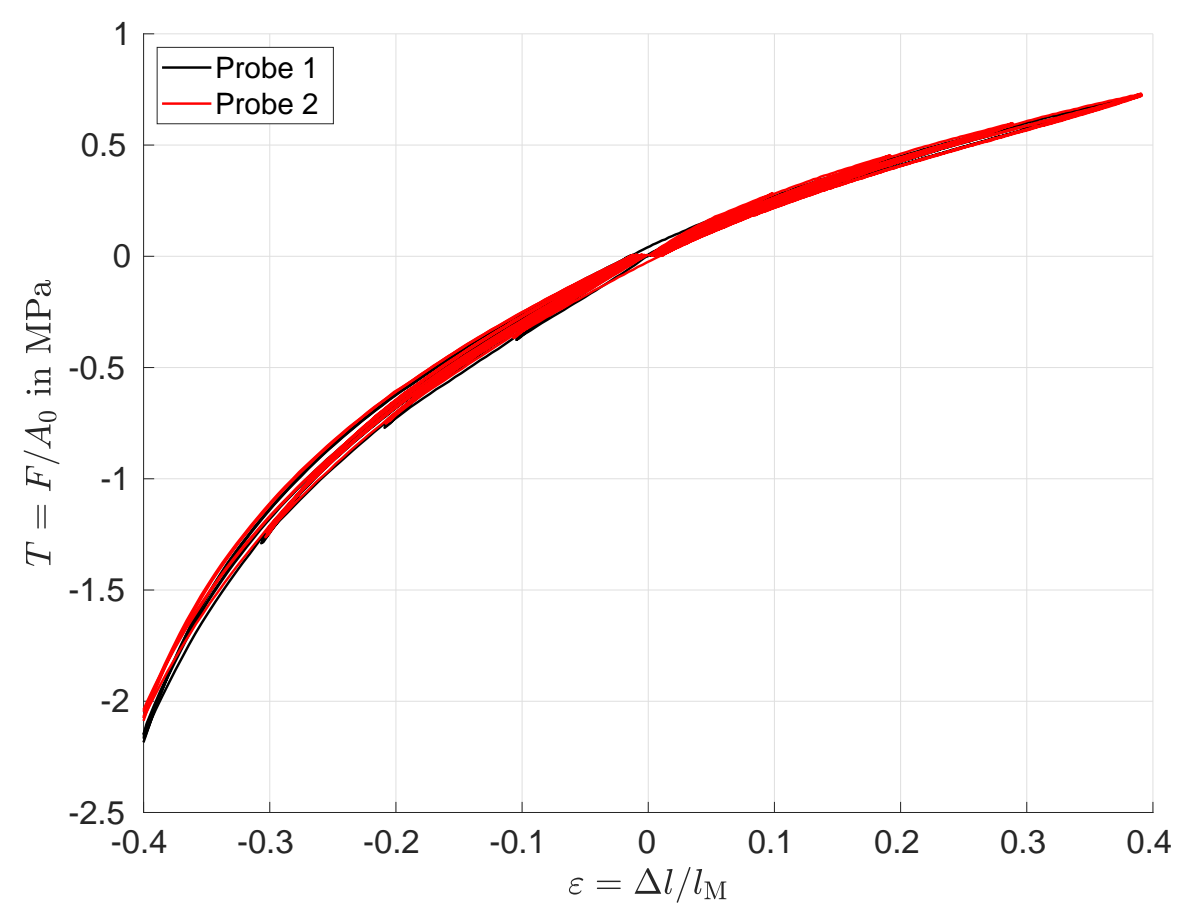

Abb. 3.60 - Vergleich von Druck-Zug (schwarz) und Zug-Druckversuchen (rot) (Messungen für gefüllten NR), Messsystem DIC. Die Abbildung ist in leicht modifizierter Form Kanzenbach u. a. (2018) entnommen. Wiederverwendung mit Genehmigung. (c) 2018 Elsevier Ltd. 
im Druckbereich einen nicht zu vernachlässigenden Einfluss auf den Zugbereich hat. Dieser Effekt, der die Steifigkeit in der entgegengesetzten Belastungsrichtung herabsetzt, wird auch als belastungsinduzierte Anisotropie bezeichnet (vgl. Ihlemann 2003, Wulf 2016). Im Vergleich dazu scheint die Vorkonditionierung im Zugbereich (blauer Verlauf) nur einen sehr geringen Einfluss auf den Druckbereich auszuüben. Die belastungsinduzierte Anisotropie tritt hier wesentlich abgeschwächt auf. Im Gegensatz zu symmetrischen Scherversuchen (vgl. Wulf 2016), wo sich im stationären Zustand eine Punktsymmetrie der beiden Scherrichtungen herausbildet, ist diese Symmetrie im einachsigen Zug-Druckversuch nicht wiederzufinden. Ein Grund dafür ist der Einfluss der Halterungen im Druckbereich, welche die Homogenität im Messbereich leicht beeinflussen. Der Effekt der belastungsinduzierten Anisotropie ist in Abbildung 3.60 aufgrund der sehr gering ausgeprägten Hysterese nicht klar detektierbar. Der Vollständigkeit halber wurde aber der gleiche Versuchsplan wie bei gefülltem EPDM gefahren.

\section{Grenzen gestufter Zug-Druckversuche}

In diesem Abschnitt soll der Einfluss einer starken Vorkonditionierung im Zugbereich auf den Druckbereich hin untersucht werden. Hintergrund dieser Charakterisierung ist, dass der Probekörper nach großen Deformationen bleibende Dehnungen aufweist, was mit einer Veränderung der Ausgangsgeometrie einhergeht. Ziel des Versuchs ist es, zu überprüfen, wie weit der Probekörper zunächst im Zugbereich belastet werden darf, um anschließend problemlos eine Druckbeanspruchung realisieren zu können. In der Abbildung 3.61 ist ein Multi-Hystereseversuch bis zu einer Zugbeanspruchung von $140 \%$ dargestellt. Aus der Abbildung wird deutlich, dass die realisierten Zugbelastungen zu keiner signifikanten Änderung des Druckzustandes führen. Für Zugbeanspruchungen $>140 \%$ wird der Mittelpunktversatz für gefüllten Naturkautschuk und die damit einhergehende Abweichung der homogenen Druckdeformation signifikant größer, weshalb für diese Versuchsmethodik eine Grenze empfohlen wird.

\section{Extreme Stauchungen}

Abschließend soll untersucht werden, inwieweit sich mit dem entwickelten ProbekörperSetup und der neuen Messstrategie extreme Stauchungen (Stauchungen bis $70 \%$ ) realisieren lassen. In Abschnitt 3.3 .7 wurden dazu die theoretischen Grundlagen erläutert. Nachfolgend soll die experimentelle Validierung mit Hilfe der neuen Messstrategie für gefüllten EPDM und gefüllten Naturkautschuk durchgeführt werden. Für die Bestimmung der Umfangsstreckung wird ein Extensometer in Umfangsrichtung eingesetzt. Aufgrund der optischen Auswertung mittels DIC bei extremen Deformationen wird der Versuchsablauf so konzipiert, dass nicht gleich die maximale Verschiebung angefahren wird, sondern eine gestufte Belastung erfolgt. Dadurch ist sichergestellt, dass bei eventuellem Versagen der Facettenauswertung (die ideale Facette hat ein möglichst stochastisches Muster) vorherige Druckstufen sauber detektiert werden können (Korrelation von Stufe zu Stufe). 


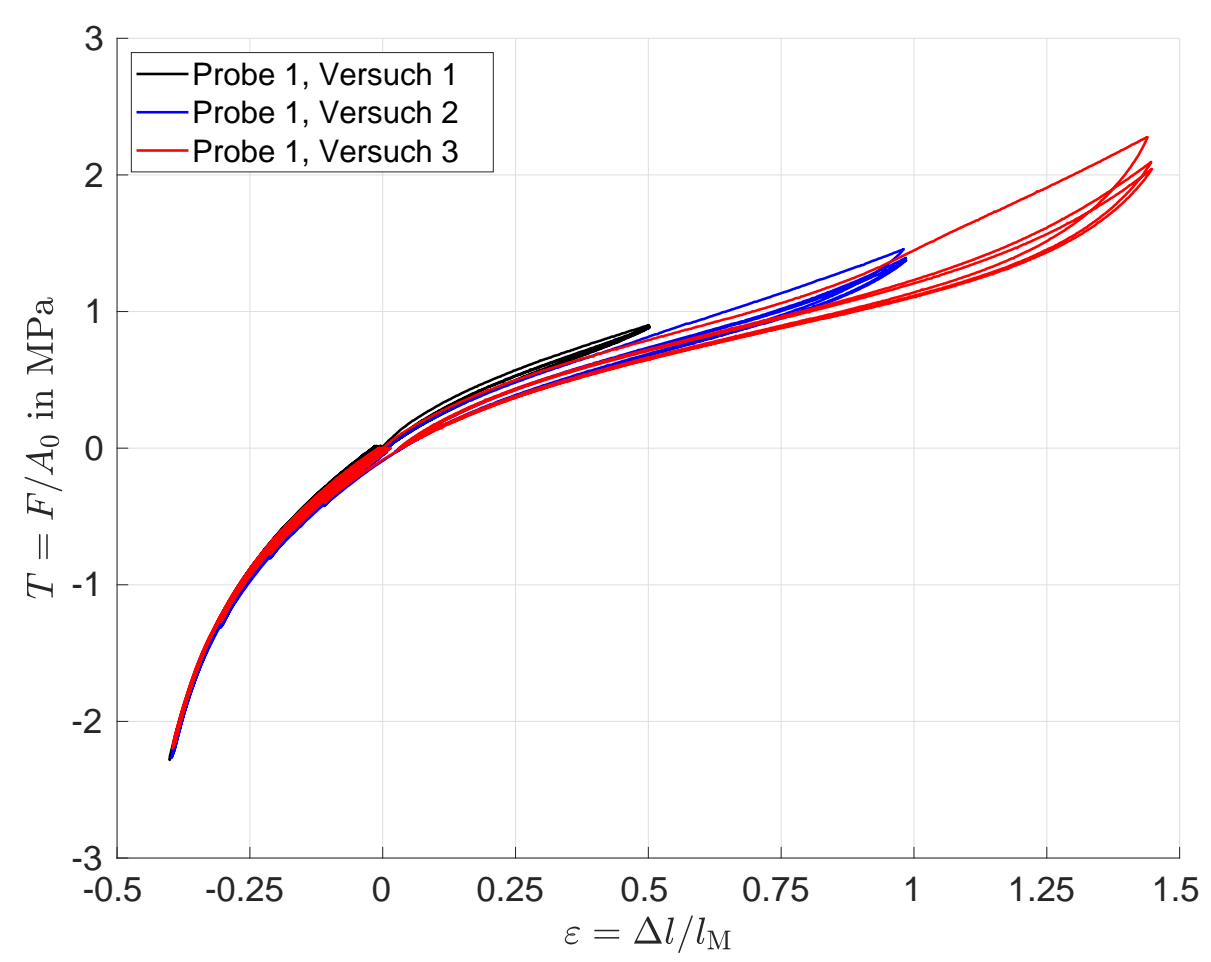

Abb. 3.61 - Multi-Hystereseversuche (Messungen für gefüllten NR), Messsystem DIC (Messungen aus der Arbeit von Oelsch (2017) durchgeführt an der Professur Festkörpermechanik, TU Chemnitz)

In der Abbildung 3.62 sind die extremen Stauchungen für gefüllten EPDM dargestellt. Aus der Abbildung wird deutlich, dass sich diese bis einschließlich $72 \%$ realisieren lassen. Dieses Ergebnis unterstreicht das Potenzial des neuen Probekörper-Setups und eröffnet damit ganz neue Anwendungsmöglichkeiten in der Materialcharakterisierung. Zur Validierung der neuen Messstrategie werden drei verschiedene Probekörper miteinander verglichen. Die Abbildung 3.62 zeigt für die durchgeführten Versuche eine gute Reproduzierbarkeit. Weiterhin ist in der Abbildung 3.62 ein Polardiagramm eingezeichnet, das die Entwicklung des Mittelpunktversatzes der Probekörper beschreibt. Obwohl der Probekörper bis einschließlich $72 \%$ gestaucht wird, beträgt der maximale Mittelpunktversatz nur $u_{\mathrm{M}}=0.4 \mathrm{~mm}$. Dieser Wert, der im Vergleich zur maximalen Durchmesseraufweitung von ca. $25 \mathrm{~mm}$ sehr gering ist, bestätigt die Qualität des gesamten Probekörper-Setups einschließlich Messmethodik.

Als nächstes werden extreme Stauchungen für gefüllten Naturkautschuk durchgeführt. In der Abbildung 3.63 ist der gestufte Druckversuch dargestellt. Im Vergleich zu gefülltem EPDM fallen neben den kleineren Hysteresen für gefüllten Naturkautschuk die größeren Abweichungen im Spannungs-Dehnungsdiagramm auf. Um die Abweichungen besser interpretieren zu können, soll im Folgenden der Mittelpunktversatz im Polardiagramm näher betrachtet werden. Hier wird zunächst deutlich, dass die absoluten Werte wesentlich größer ausfallen als für gefüllten EPDM. Der größte Mittelpunktversatz beträgt $u_{\mathrm{M}}=1.2 \mathrm{~mm}$, 


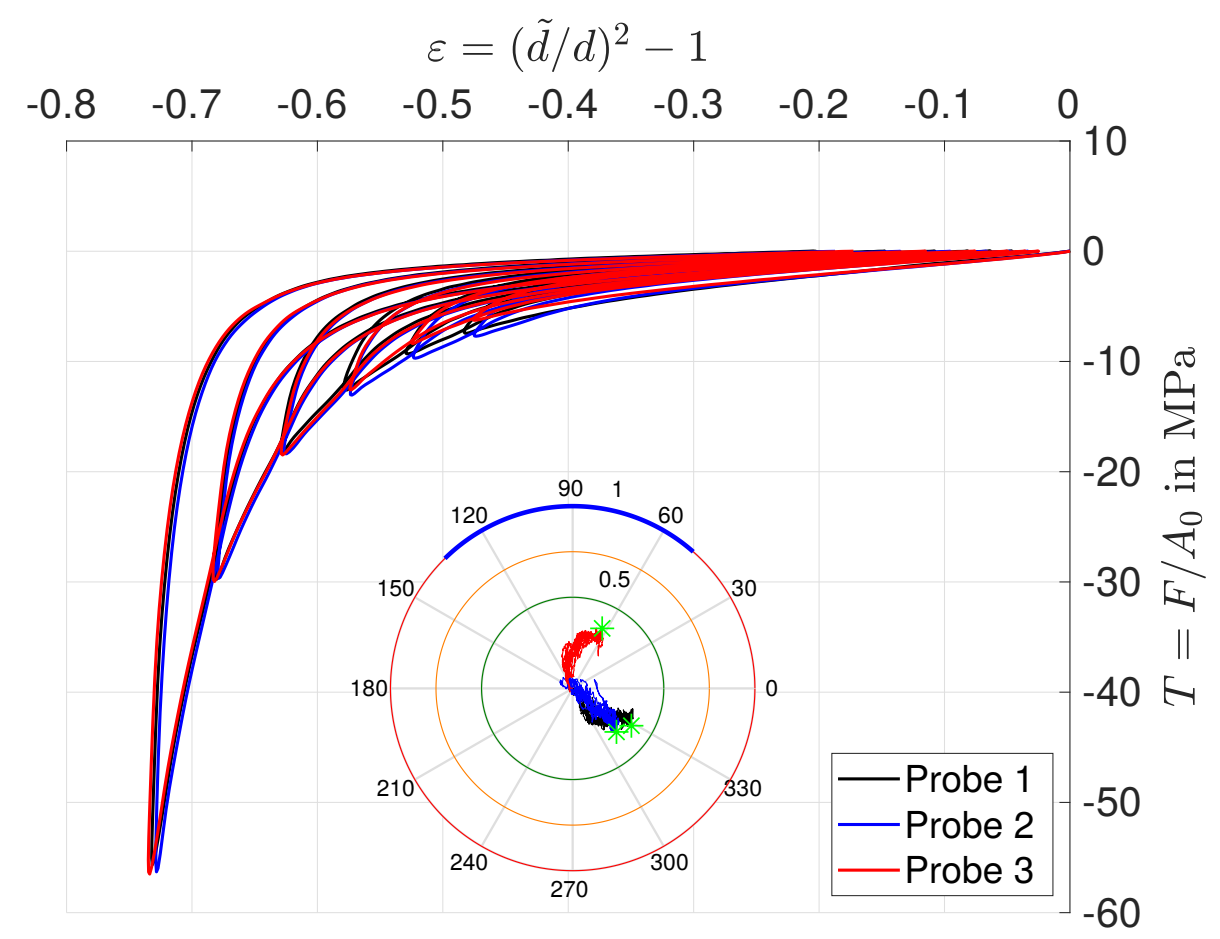

Abb. 3.62 - Darstellung der extremen Stauchung (Messungen für gefüllten EPDM), ermittelt über die Messung der Umfangsstreckung via DIC

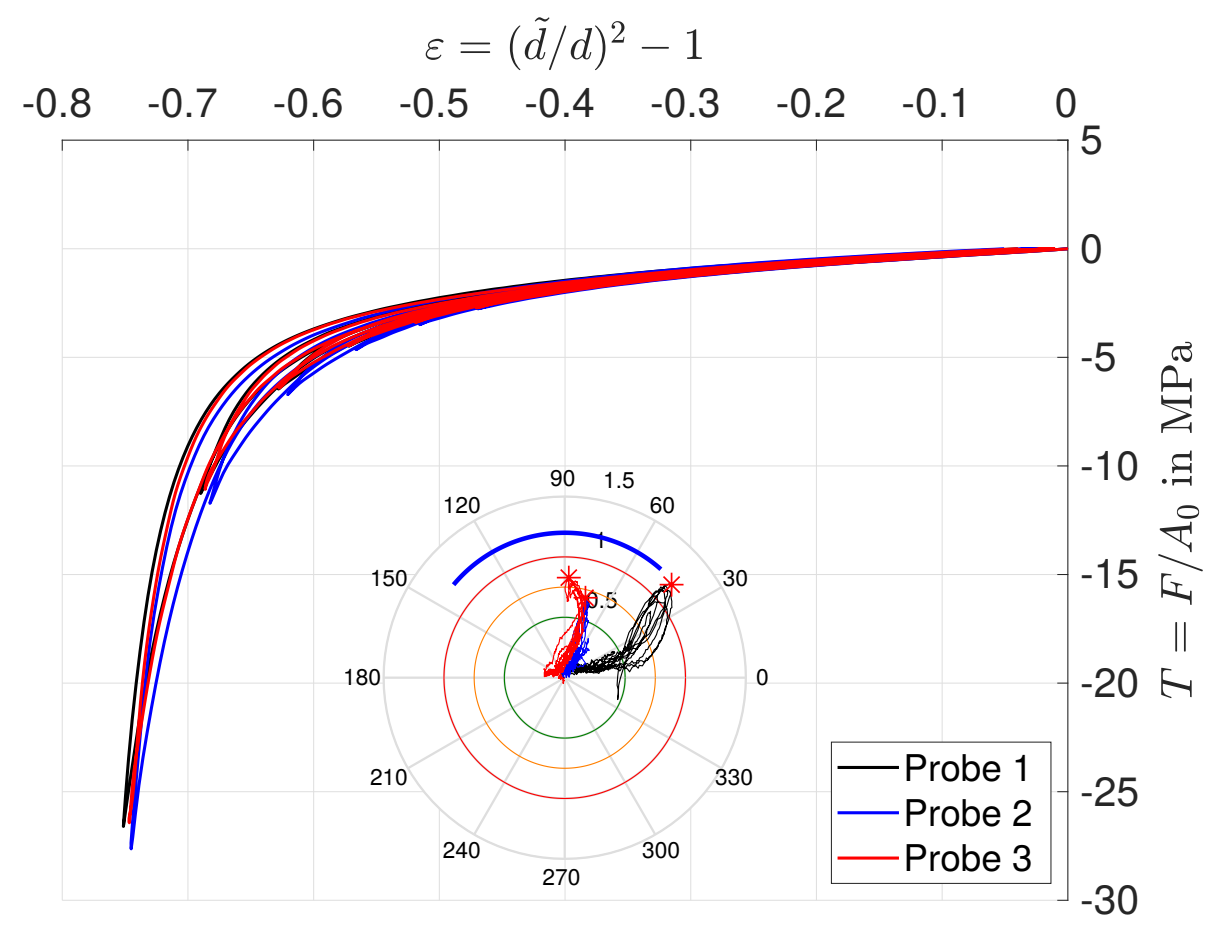

Abb. 3.63 - Darstellung der extremen Stauchung (Messungen für gefüllten NR), ermittelt über die Messung der Umfangsstreckung via DIC

was aber im Vergleich zur maximalen Durchmesseraufweitung immer noch verhältnismäBig gering ist. Nichtsdestotrotz scheint gefüllter Naturkautschuk viel sensitiver auf extre- 
me Stauchungen zu reagieren als gefüllter EPDM. Für den ersten gestuften Druckversuch (schwarzer Verlauf), der die größten Abweichungen aufzeigt, sollen im Folgenden verschiedene Druckstufen näher betrachtet werden. In der Abbildung 3.64 sind die verschiedenen Druckstufen als Bilder dargestellt (von links nach rechts, von oben nach unten). Die
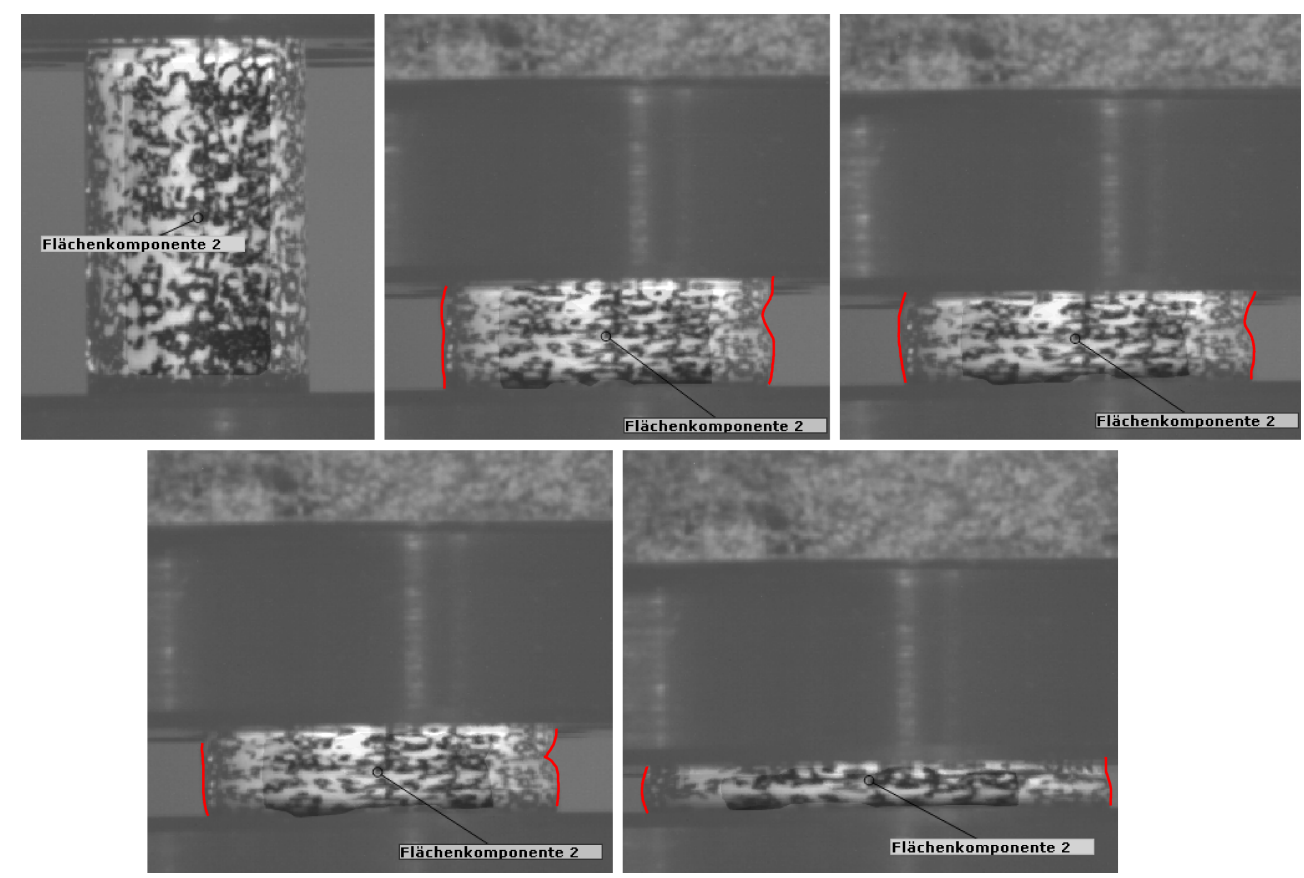

Abb. 3.64 - Entwicklung der lokalen Faltenbildung bei extremen Stauchungen für gefüllten NR

Außenkontur wurde zur besseren Veranschaulichung mit einer roten Linie nachgezeichnet. Anhand der Abbildung 3.64 wird deutlich, dass sich mit zunehmender Stauchung an der rechten Außenkontur eine lokale Falte bildet, die bei noch größeren Stauchungen zugedrückt wird. Diese Faltenbildung ist reproduzierbar für alle durchgeführten extremen Stauchungen mit gefülltem Naturkautschuk (MAA50-00). Es sei an dieser Stelle darauf hingewiesen, dass hier bewusst ein Druckversuch ausgewählt worden ist, bei dem die Faltenbildung seitlich detektiert werden kann. Diese Materialinhomogenität, die bisher nur bei gefülltem Naturkautschuk (MAA50-00) im Zusammenhang mit extremen Stauchungen beobachtet werden kann, erklärt die Abweichungen im Spannungs-Dehnungsdiagramm, siehe Abbildung 3.63. Gefüllter Naturkautschuk scheint bei solchen extremen Belastungen sehr sensitiv auf lokale Störungen zu reagieren, welche leicht im Material durch den Herstellungsprozess entstehen können. Beispielsweise können bei der Herstellung von MAA50-00 Endmischungseffekte auftreten, die aus dem Compound der zwei Basispolymere resultieren. Es bleibt in weiterführenden Arbeiten zu klären, inwieweit sich diese Materialinhomogenität beheben lässt. Zusammenfassend kann gesagt werden, dass sich extreme Stauchungen realisieren lassen, trotz der Einschränkungen bei gefülltem Naturkautschuk. Besonders bei gefülltem EPDM werden sehr gut reproduzierbare Messergebnisse erzielt. 


\section{Entwicklung eines Scherprobekörpers für präzise Schermessungen}

In diesem Kapitel erfolgt die numerische Entwicklung eines präzisen Scherprobekörpers zur Realisierung homogener einfacher Scherungen. Ein besonderer Schwerpunkt liegt dabei auf der Entwicklung eines speziellen Klemmdesigns, welches zur Ausbildung nahezu homogener Scherdeformationen führt. Im Anschluss erfolgt die experimentelle Validierung und der Abgleich mit Simulationsergebnissen.

\subsection{Grundlegendes zu einfachen Scherversuchen}

Im vorherigen Kapitel wurde bereits ein neu konzipiertes Probekörper-Setup vorgestellt, welches nun erstmalig hochpräzise einachsige Zug-/Druckversuche ermöglicht. Dabei konnten grundlegende elastomertypische Effekte auch bei extremen Stauchungen untersucht werden. Neben der einachsigen Zug-/Druckbeanspruchung spielen aber auch andere Beanspruchungsarten, wie die einfache Scherung, eine elementare Rolle bei der phänomenologischen Untersuchung technischer Elastomere. Beispielsweise können hier Aussagen zur belastungsinduzierten Anisotropie getroffen werden. Neben der phänomenologischen Untersuchung grundlegender Effekte werden die neu entwickelten homogenen Probekörper auch für die Identifikation von Materialparametern benötigt. Zum Beispiel treten bei Fahrwerksbuchsen eine Vielzahl von Belastungsarten auf, wofür Anpassungen an verschiedenen homogenen Probekörpern benötigt werden (vgl. Schellenberg 2017). Um unterschiedliche Belastungsarten in einem Abbildungsraum miteinander vergleichen zu können, empfiehlt sich eine Darstellung mittels der Hauptinvarianten $I_{1}$ und $I_{2}$ :

$$
I_{1}(\underline{\underline{C}})=\lambda_{1}^{2}+\lambda_{2}^{2}+\lambda_{3}^{2}, \quad I_{2}(\underline{\underline{C}})=\lambda_{1}^{2} \lambda_{2}^{2}+\lambda_{2}^{2} \lambda_{3}^{2}+\lambda_{1}^{2} \lambda_{3}^{2}
$$

Da technische Elastomere in erster Näherung als inkompressibel angesehen werden können, kann die dritte Hauptinvariante zu $I_{3}(\underline{\underline{F}})=1$ gesetzt werden. Die Hauptstreckungen $\lambda_{1}, \lambda_{2}$ und $\lambda_{3}$ ergeben sich je nach Belastungsrichtung wie folgt:

- einachsiger Zug-/Druckversuch mit $\lambda_{1}=\lambda$ und $\lambda_{2}=\lambda_{3}=\frac{1}{\sqrt{\lambda}}$ 
- biaxialer Zugversuch mit $\lambda_{1}=\lambda_{2}=\lambda$ und $\lambda_{3}=\frac{1}{\lambda^{2}}$

- ebener Zugversuch (engl. pure shear) mit $\lambda_{1}=\lambda, \lambda_{2}=1$ und $\lambda_{3}=\frac{1}{\lambda}$

- einfacher Scherversuch mit dem Schermaß $s: \lambda_{1,2}=\sqrt{1+\frac{s^{2}}{4}} \pm \frac{s}{2}$ und $\lambda_{3}=1$.

In der Abbildung 4.1 sind für einen Materialpunkt verschiedene Beanspruchungsarten dargestellt. Anhand der Abbildung wird deutlich, dass der einachsige Zug- bzw. Druck

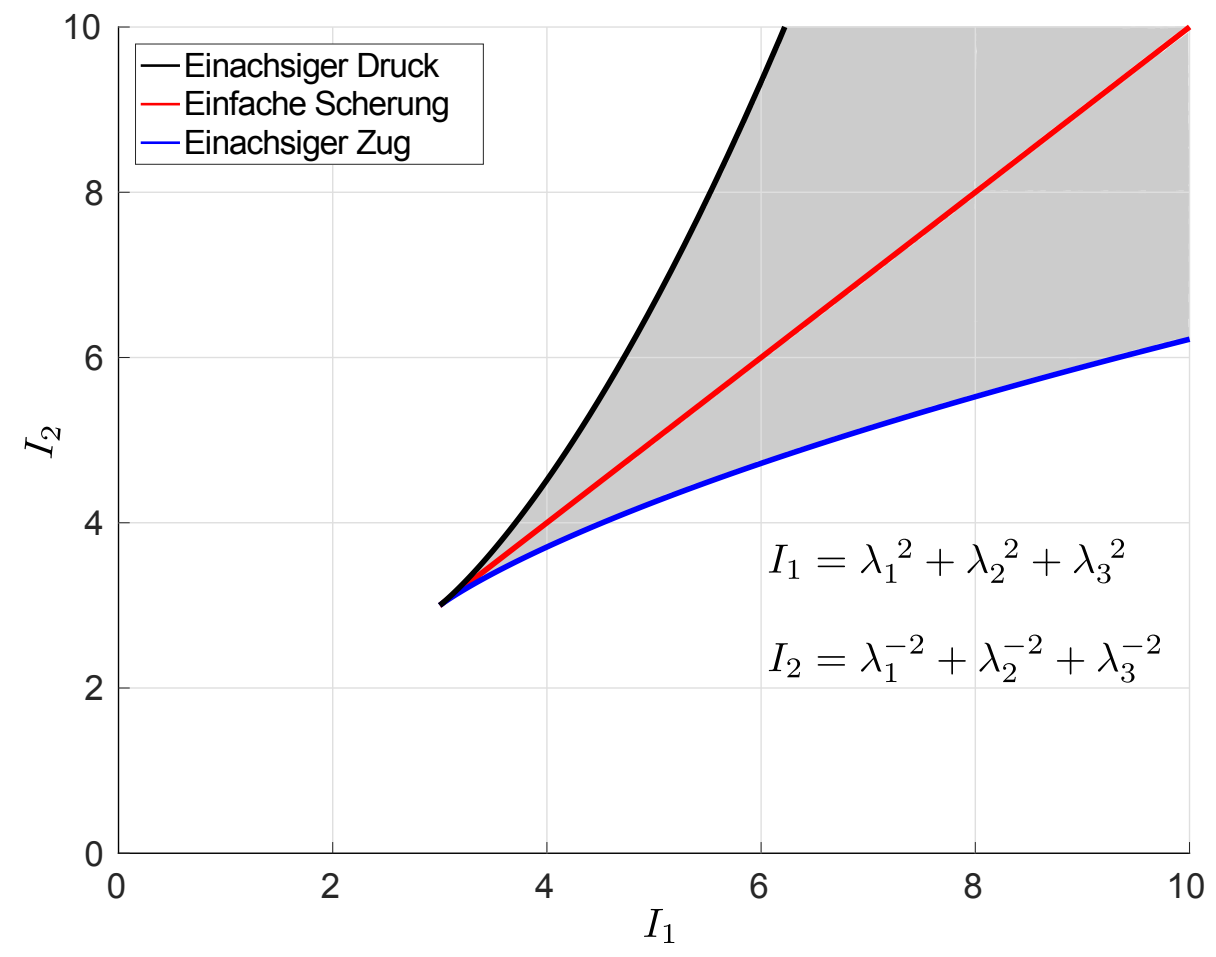

Abb. 4.1 - Darstellung der Hauptinvarianten $I_{1}$ und $I_{2}$ bei verschiedenen Beanspruchungsarten (vgl. Krawietz 1986)

den Grenzbereich angibt. Die graue Fläche kennzeichnet dabei den zulässigen Bereich möglicher Beanspruchungsarten. Die einfache Scherung stellt insofern einen Sonderfall als Beanspruchungsart dar, weil hier die Hauptinvarianten $I_{1}$ und $I_{2}$ identisch sind. Insbesondere bei einer Stoffgesetzanpassung sollte darauf geachtet werden, dass verschiedene Belastungsarten in der Parameteridentifikation mit berücksichtigt werden (vgl. Schellenberg 2017).

Die Hauptinvarianten können im Weiteren auch für die Definition von Fehlermaßen verwendet werden. Beispielsweise kann über die euklidische Norm der senkrechte Abstand zwischen der theoretisch vorliegenden Scherung und der aktuell vorhandenen Scherung berechnet werden. In der Gleichung $44-2$ ist das Fehlermaß $\eta_{\text {I }}$ als normierte Größe darge- 
stellt:

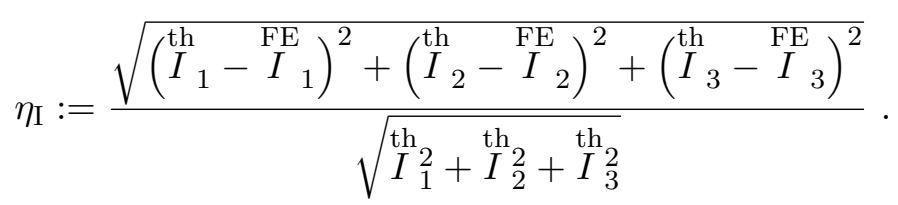

An dieser Stelle sei explizit darauf hingewiesen, dass die Invarianten für das hier definierte Fehlermaß dimensionslos sein müssen. Andernfalls ergeben sich Probleme mit der Dimension. In Abschnitt 4.4.1 erfolgt die Einführung weiterer Fehler- und Inhomogenitätsmaße, die zur Bewertung der entwickelten Scherprobekörper benötigt werden.

\subsection{Motivation für die Verwendung flächiger Scherprobekörper zur Realisierung präziser Schermessungen}

In Abschnitt 2.2.3 erfolgte der Vergleich zwischen flächigen und zylindrischen Scherprobekörpern. Dabei konnte gezeigt werden, dass flächige Scherprobekörper eine Reihe ganz prinzipieller Vorteile aufweisen. Die Wichtigsten seien hier nochmal zusammengestellt:

- homogene Alterungsuntersuchungen sind möglich

- faserverstärkte Materialien können getestet werden

- Scherversuche mit Normalkraft Null durchführbar

- anisotrope Einflüsse (Vorreckung auf Zug) können untersucht werden

- Verwendung von Serienmaterial in Matten

Nachteilig ist dabei das Risiko von Rutsch- und Beuleffekten (vgl. Gross u. a. 2007). Dieser Tatsache kann aber durch konstruktive Maßnahmen bzw. einer geeigneten Dimensionierung der Probekörpergeometrie begegnet werden. In Abschnitt 4.4.1 werden deshalb Störungsrechnungen zur Untersuchung der Knickstabilität durchgeführt.

Nachdem die Vorzüge von flächigen Scherprobekörpern dargelegt sind, besteht nun eines der Hauptziele in der Entwicklung eines geeigneten Klemmdesigns, welches zur Ausbildung nahezu homogener einfacher Schedeformationen führt. Da gerade die Lasteinleitung dabei eine große Herausforderung darstellt, soll im nächsten Abschnitt gesondert darauf eingegangen werden. Das andere Ziel besteht in der Realisierung großer Scherdeformationen $F_{x y} \geq 2.0$.

\subsection{Grundproblem Lasteinleitung}

Im Folgenden wird auf eine Grundproblematik der einfachen Scherdeformation hingewiesen: die gleichmäßige Lasteinleitung. In der Abbildung 4.2 ist die einfache Scherung unter Annahme idealer Inkompressibilität für einen Einheitswürfel schematisch dargestellt. 


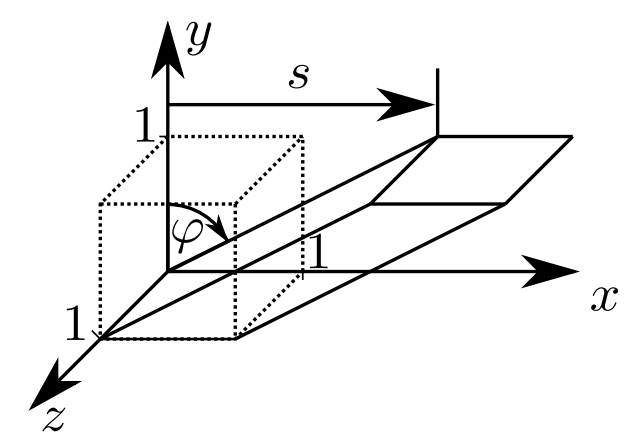

Abb. 4.2 - Darstellung der einfachen Scherung unter Annahme idealer Inkompressibilität

Um diesen Deformationszustand auch mit einem realen flächigen Probekörper zu erzielen, bedarf es spezieller Anforderungen bzgl. der Lasteinleitung. Die dabei zur Verfügung stehenden Grundprinzipien lauten wie folgt:

- stoffschlüssige Verbindung (Kleben/Anvulkanisieren)

- kraftschlüssige Verbindung (mechanische Klemmung durch Normalkräfte/Reibung)

- formschlüssige Verbindung (Profilleisten, Löcher und Stifte) .

Eine stoffschlüssige Verbindung mittels Kleben oder Anvulkanisieren hat den prinzipiellen Vorteil, dass die Last über die Stirn- oder Deckflächen eingeleitet werden kann. Der große Nachteil besteht aber darin, dass die Grenzschicht (Elastomer/Metall-Verbund) zum einen große Beanspruchungen des Elastomers aufnehmen muss und zum anderen starr an das Metall angebunden ist (vgl. Ballhorn 2007). Dadurch treten im Übergangsbereich örtlich sehr große Spannungen auf, die alle durch die Bindeschicht aufgenommen werden müssen. Somit bildet der Kontaktbereich zwischen Elastomer und Metall immer eine Schwachstelle. Ein weiterer prinzipieller Nachteil besteht darin, dass der Vernetzer (der im Bindemittel vorhanden ist) in die Kautschukmischung diffundiert und es dadurch zu Materialirritationen in der Grenzschicht kommt. In der Veröffentlichung von Ballhorn (2007) sind weitere Einflussparameter beschrieben, die sich nachteilig auf den Elastomer/Metall-Verbund auswirken können. Demgegenüber besteht beim Anvulkanisieren der große Nachteil, dass der Probekörper einem Schrumpfprozess ausgesetzt ist. Hinzu kommt, dass durch den Abkühlprozess nach der Vulkanisation Eigenspannungen auftreten, die zu einer Beeinträchtigung der Homogenität im Messbereich führen. Dieser Verzug wirkt sich weiterhin nachteilig auf die Versuchsvorrichtung aus und erschwert den kraft- bzw. momentenfreien Einbau.

Eine kraftschlüssige Verbindung kann beispielsweise durch eine mechanische Klemmung der Elastomermatte zwischen zwei Metallleisten realisiert werden. Über die dabei auftretenden Normalkräfte kann ein Reibschluss erzeugt werden. Der Nachteil, der sich hieraus ergeben kann, liegt in dem Umstand, dass das Material innerhalb des Klemmbereiches in den relevanten Messbereich verdrängt wird. Ein ganz anderes Problem kann dann entstehen, wenn der Reibschluss nicht mehr gewährleistet ist und die Elastomermatte zwischen 
den Metallleisten rausrutscht.

Als letztes soll auf die Vor- und Nachteile einer formschlüssigen Verbindungstechnik eingegangen werden. Hier ergeben sich eine Reihe interessanter Möglichkeiten. Beispielsweise kann eine formschlüssige Verbindung dadurch realisiert werden, dass sowohl die Elastomermatte selbst als auch die umschließende Metallleiste einer Profilform folgen. Eine andere Möglichkeit könnte beispielsweise aus einer Kombination aus Kraft- und Formschluss bestehen, indem eine spezielle Profilkontur in die Elastomermatte gedrückt wird. Die Nachteile, die sich hieraus ergeben können, wären beispielsweise eine komplexere Probenpräparation und gegebenenfalls eine separate Vulkanisationsform, falls die Elastomermatte ein spezielles Profil aufweisen muss. Zusammenfassend lässt sich sagen, dass für die Realisierung präziser Schermessungen auf eine kraft- und/oder formschlüssige Verbindung zurückgegriffen werden soll.

\subsection{Numerische Entwicklung einer Schervorrichtung zur Realisierung präziser Schermessungen}

\subsubsection{Fehler- und Inhomogenitätsmaße}

Aufbauend auf den Erkenntnissen aus Abschnitt 3.3.4 sollen im Folgenden die verschiedenen Fehler- und Inhomogenitätsmaße zur Bewertung der Scherprobekörper definiert werden. Diese Maße werden dann zunächst an einem idealisierten Scherprobekörper (Scherprobekörper mit idealen Randbedingungen) getestet. Die Maße sollen dabei in der Weise definiert werden, dass sie zum einen Auskunft über die Abweichung zur theoretisch vorliegenden Spannung liefern, zum anderen die Abweichungen zur Homogenität selbst angeben. In der Abbildung 4.2 wurde die einfache Scherung unter Annahme idealer Inkompressibilität für einen Einheitswürfel bereits dargestellt. Der zugehörige Deformationsgradient nimmt dabei folgende Form an:

$$
\left[F_{a b}\right]=\left[\begin{array}{ccc}
1 & s & 0 \\
0 & 1 & 0 \\
0 & 0 & 1
\end{array}\right] \text { mit: } s=\tan (\varphi)
$$

Die Größe $s$ beschreibt dabei das Schermaß, welches auch durch den Tangens des Scherwinkels angegeben werden kann (vgl. Ihlemann 2003). Über den Deformationsgradienten und den Cauchy-Spannungstensor kann dann der 1. Piola-Kirchhoff-Spannungstensor berechnet werden (siehe Gleichung (2-28)).

$$
\left[\sigma_{a b}\right]=\left[\begin{array}{ccc}
\sigma_{x x} & \sigma_{x y} & 0 \\
\sigma_{y x} & \sigma_{y y} & 0 \\
0 & 0 & \sigma_{z z}
\end{array}\right] \Rightarrow\left[T_{a b}\right]=\left[\begin{array}{ccc}
\sigma_{x x}-s \sigma_{x y} & \sigma_{x y}-s \sigma_{y y} & 0 \\
\sigma_{y x} & \sigma_{y y} & 0 \\
0 & 0 & \sigma_{z z}
\end{array}\right]
$$


Dabei ist zu berücksichtigen, dass der 1. Piola-Kirchhoff-Spannungstensor im allgemeinen unsymmetrisch ist (vgl. Gleichung (4-4)). Als nächstes wird das Spannungsmaß ؟ eingeführt, welches sich aus der Differenz des aktuell vorliegenden Cauchy-Spannungstensors $\underset{\underline{\sigma}}{\stackrel{\mathrm{F}}{\sigma}}$ (aus FE-Simulation) und des theoretisch vorhandenen Spannungstensors $\stackrel{\text { th }}{\underline{\sigma}}$ (bei gleichem Schermaß s) zusammensetzt. Über die Definition der Von-Mises-Vergleichsspannung kann aus dem Spannungsmaß $\leqq$ ein skalares Vergleichsmaß $\varsigma_{\text {eqv }}$ berechnet werden. Das lokale Fehlermaß $\eta_{\mathrm{s}}$ ergibt sich dann aus dem Verhältnis der berechneten Vergleichsspannung $\varsigma_{\text {eqv }}$ und der theoretisch vorhandenen Vergleichsspannung $\stackrel{\text { th }}{\sigma}_{\text {eqv }}$ (vgl. Gleichung $(3-47)$ ):

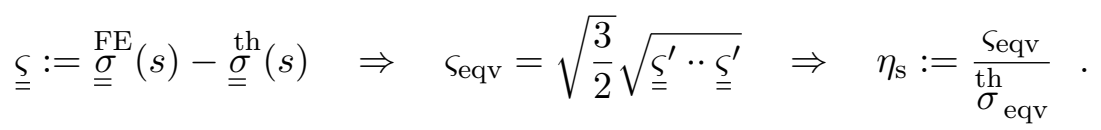

Über die Subroutine PLOTV in der FE-Software Msc.Marc wird die theoretische Lösung $\stackrel{\text { th }}{\underline{\underline{\sigma}}}$ und das entwickelte Fehlermaß $\eta_{\mathrm{s}}$ berechnet. Als Stoffgesetz findet das hyperelastische Yeoh-Modell mit schwacher Kompressibilität Anwendung (vgl. Gleichung (2-50)). Die Materialparameter sind der Veröffentlichung von Schellenberg u. a. (2012) entnommen (vgl. Tabelle 4.1). Für weiterführende Informationen zur Implementierung selbst entwickelter

\begin{tabular}{|c|c|c|c|}
\hline$c_{10}$ in $\mathrm{MPa}$ & $c_{20}$ in $\mathrm{MPa}$ & $c_{30}$ in $\mathrm{MPa}$ & $K$ in $\mathrm{MPa}$ \\
\hline 0.359 & -0.0115 & 0.00454 & 5000 \\
\hline
\end{tabular}

Tabelle 4.1 - Materialparameter für Yeoh (vgl. Schellenberg u.a. 2012)

Ausgabegrößen über die Subroutine PLOTV wird auf das Manual der Msc. Software 2017 Volume A: Theory and User Information verwiesen. In der Abbildung 4.3 ist das lokale Fehlermaß $\eta_{\mathrm{s}}$ für den idealisierten Scherprobekörper dargestellt. Anhand des Farbverlaufes wird deutlich, dass die einfache Scherung entlang der Scherrichtung $x$ sehr gut übertragen werden kann. Die roten Außenbereiche (in der $x z$-Ebene) veranschaulichen dabei sehr deutlich den Kontrast zwischen ungeschertem und geschertem Material. Im Vergleich zum mittleren Bereich sind an den freien Rändern größer werdende Fehlermaße zu verzeichnen. Inwiefern diese freien Ränder (oben und unten) einen Einfluss auf das globale Verhalten ausüben, soll mit Hilfe des nächsten Fehlermaßes näher untersucht werden.

Im Folgenden wird dazu das globale Fehlermaß $\eta_{\mathrm{T}}$ eingeführt, welches den relativen Fehler zwischen vorliegender und theoretischer 1. Piola-Kirchhoff-Spannung für die einfache Scherung angibt (in Anlehnung an Gleichung (3-42):

$$
\eta_{\mathrm{T}}:=\left|\frac{\stackrel{\mathrm{FE}}{T}_{y x}(s)-\stackrel{\mathrm{th}}{T}_{y x}(s)}{\stackrel{\mathrm{th}}{T}_{y x}(s)}\right| \quad \text { mit: } \quad \stackrel{\mathrm{FE}}{T}_{y x}(s):=\frac{F_{x}(s)}{A_{0}} .
$$

Für die $y x$-Komponente des Spannungstensors gilt die Besonderheit: $\stackrel{\mathrm{FE}}{T}_{y x}=\stackrel{\mathrm{FE}}{\sigma}_{y x}(\operatorname{vgl}$. Gleichung (4-4)). Somit kann über die Referenzfläche $A_{0}$ und die gemessene Scherkraft $F_{x}$ 


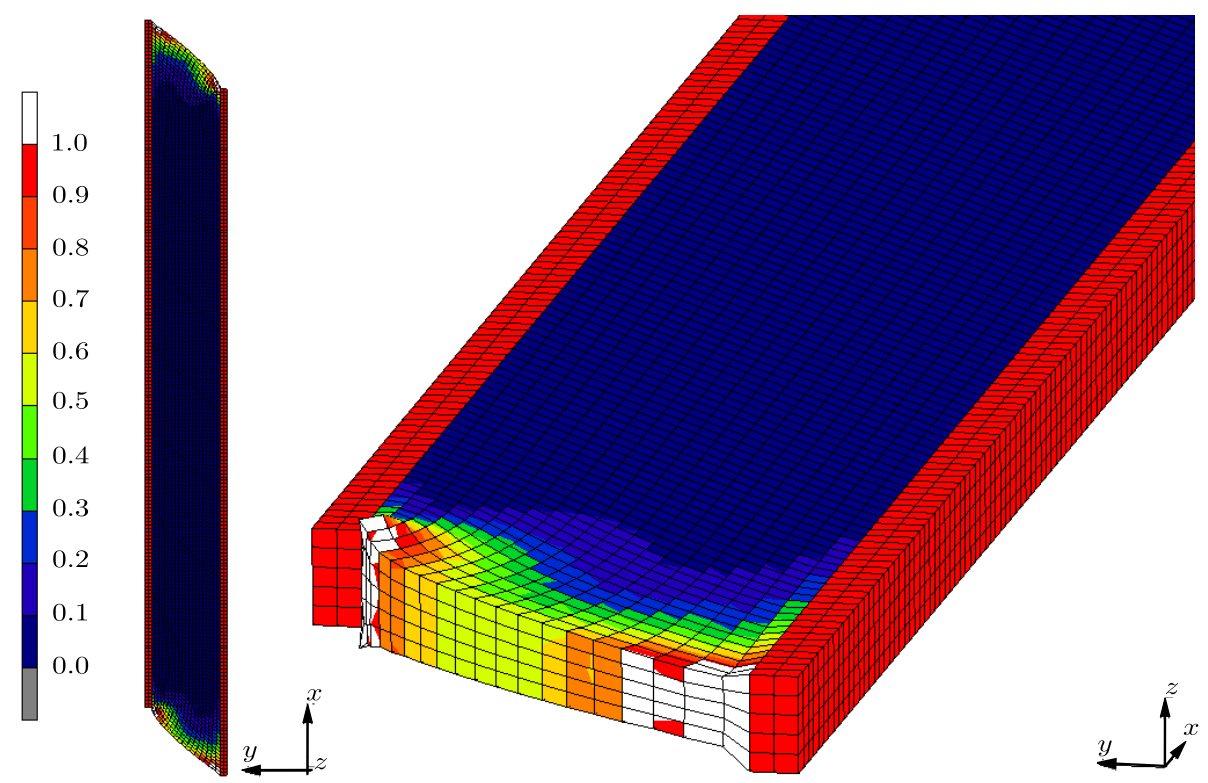

Abb. 4.3 - Darstellung des lokalen Fehlermaßes $\eta_{\mathrm{s}}$ für den idealisierten Scherprobekörper mit den Abmaßen: $10 \times 100 \times 2$ bei einem Schermaß von $s=1$

auch die Cauchy-Spannung berechnet werden. In der Abbildung 4.4 ist das SpannungsSchermaßdiagramm und in der Abbildung 4.5 das globale Fehlerdiagramm $\eta_{\mathrm{T}}$ dargestellt. Aus der Abbildung 4.4 wird ersichtlich, dass die freien Ränder des idealisierten Scherprobekörpers zu keiner signifikanten Beeinflussung des globalen Kraftverhaltens führen.

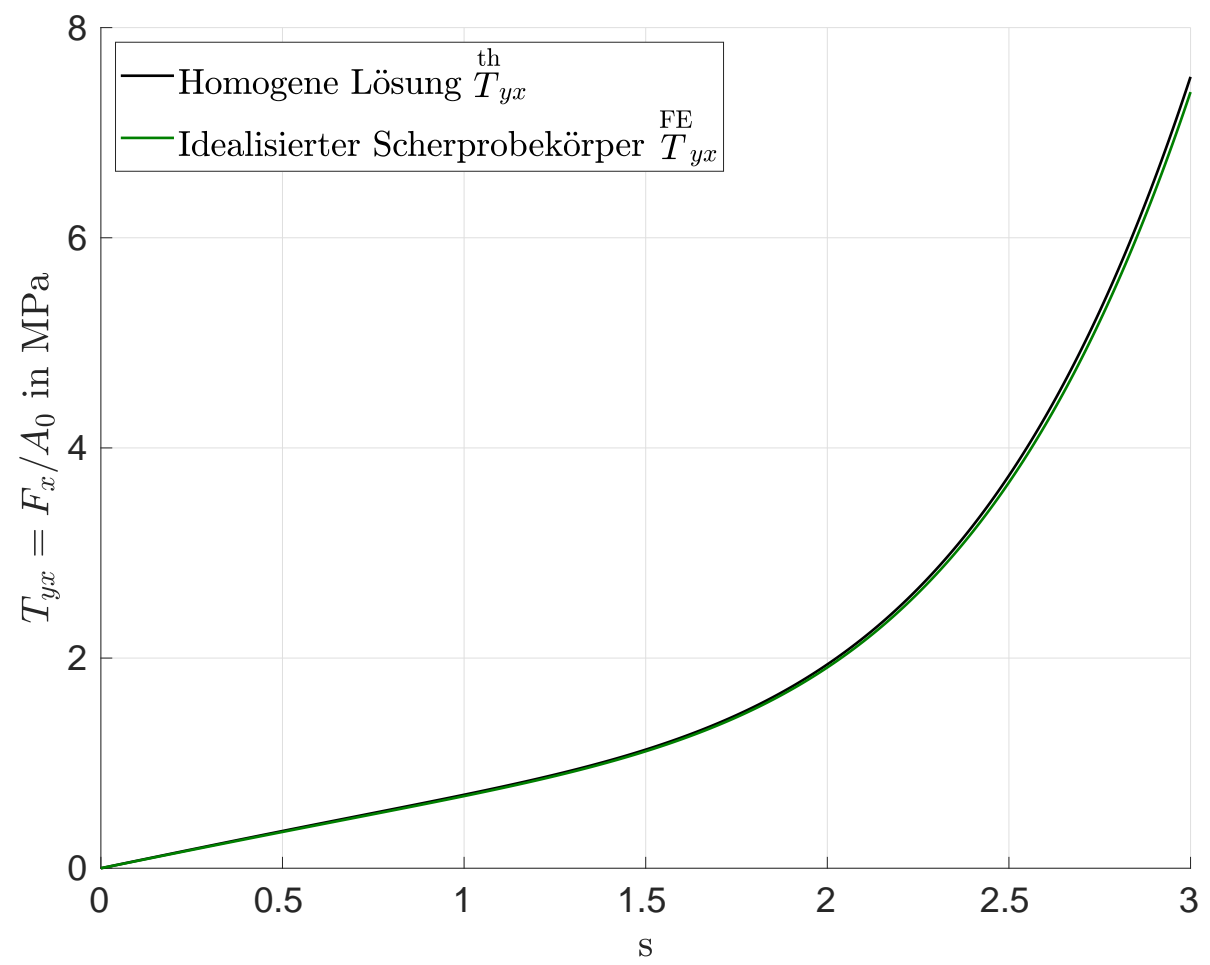

Abb. 4.4 - Spannungs-Schermaßdiagramm für den idealisierten Scherprobekörper (Simulationsergebnisse vgl. Tabelle 4.1p 


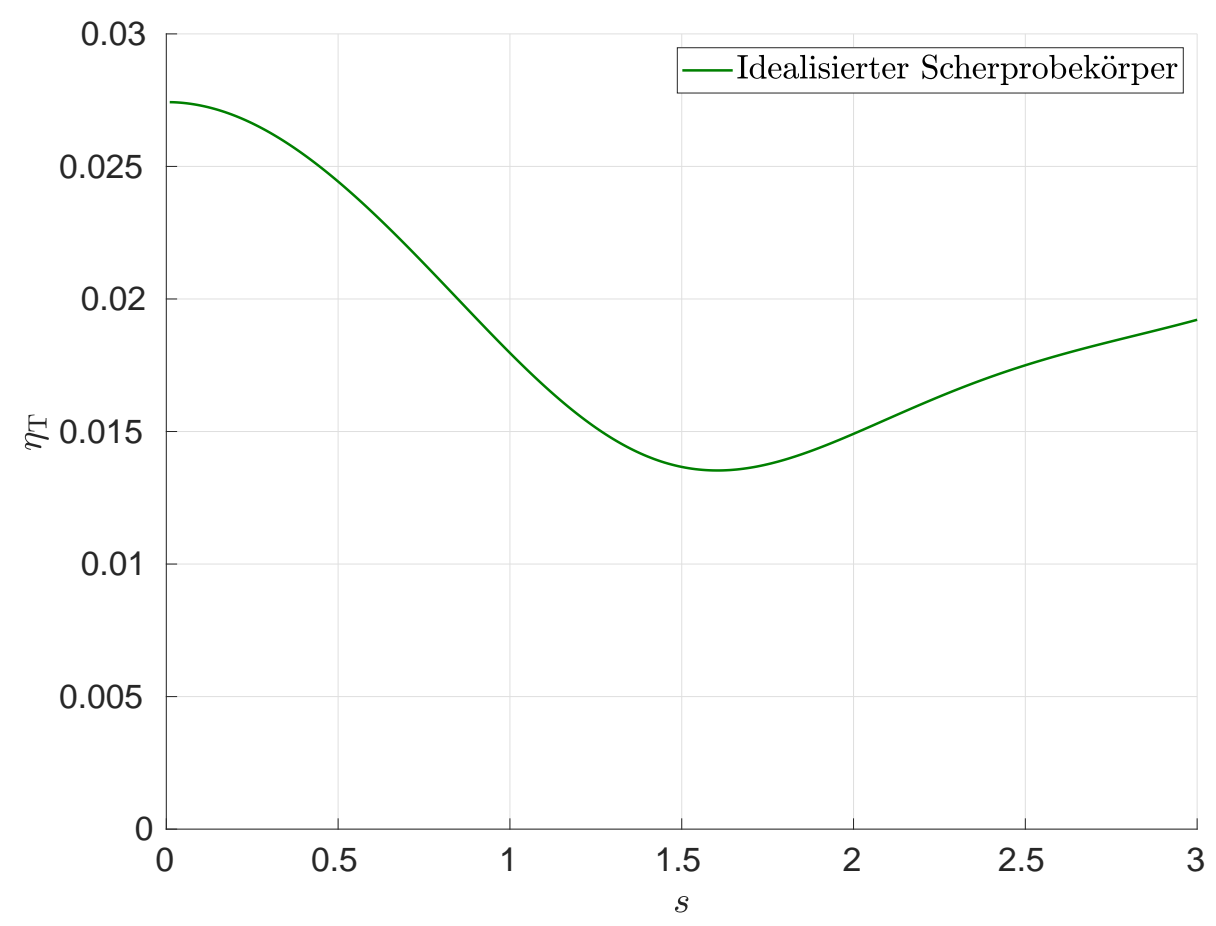

Abb. 4.5 - Globaler Messfehler $\eta_{\mathrm{T}}$ für den idealisierten Scherprobekörper (Simulationsergebnis vgl. Tabelle 4.1)

Betrachtet man dazu das globale Fehlerdiagramm genauer (vgl. Abbildung 4.5), so kann ein Fehler $\eta_{\mathrm{T}} \leq 2.75 \%$ detektiert werden. Auffallend ist, dass die Fehlergröße $\eta_{\mathrm{T}}$ über das Schermaß $s$ einen nicht monotonen Verlauf annimmt, der bei $s=1.6$ ein Minimum findet. Eine feinere Diskretisierung des freien Randes führt zu keiner prinzipiellen Änderung des Fehlerverlaufes. Da der globale Fehler, der durch die freien Ränder entsteht, verhältnismäßig gering ausfällt, soll hier nicht weiter darauf eingegangen werden. Gegebenenfalls kann in einem späteren Optimierungsschritt durch eine leichte Taillierung der freien Ränder der Fehler weiter reduziert werden. Es sei an dieser Stelle auch auf den Sachverhalt hingewiesen, dass sich die Fehler an den Rändern nicht wie in einer Reihenschaltung verhalten (vgl. Randeffekte beim Zug-/Druckprobekörper), sondern sich wie in einer Parallelschaltung aufsummieren.

Im Weiteren wird ein globales Maß definiert, welches sich zur Bewertung der Spannungsinhomogenität im Messbereich eignet. Dieses soll im Gegensatz zu den eingeführten Fehlermaßen nicht auf die Abweichung zu einer theoretisch vorhandenen Lösung Bezug nehmen, sondern die Abweichungen im Messbereich zu einem berechneten Mittelwert selbst bewerten. Dazu wird auf eine bekannte Größe aus der Statistik zurückgegriffen. Das Inhomogenitätsmaß $\xi_{\mathrm{s}}$ wird als Standardabweichung der Von-Mises-Vergleichsspannung definiert:

$$
\xi_{\mathrm{s}}:=\sqrt{\frac{1}{n-1} \sum_{i=1}^{n}\left(\sigma_{\mathrm{eqv}_{i}}-\bar{\sigma}_{\mathrm{eqv}}\right)^{2}} \quad \text { mit: } \quad \bar{\sigma}_{\mathrm{eqv}}=\frac{1}{n} \sum_{i=1}^{n} \sigma_{\mathrm{eqv}_{i}} .
$$


Die Größe $\sigma_{\text {eqv }_{i}}$ gibt dabei die Vergleichsspannung an einem Knoten $i$ an. Weiterhin beschreibt das Maß $\bar{\sigma}_{\text {eqv }}$ den Mittelwert für die Vergleichsspannung für einen ausgewählten Messbereich. In der Abbildung 4.6 ist dieser in Abhängigkeit des Schermaßes $s$ für den idealisierten Scherprobekörper (zum besseren Verständnis vergleiche Scherbereich in Abbildung 4.3 ohne roten Außenbereich) dargestellt. Anhand der Abbildung werden einige

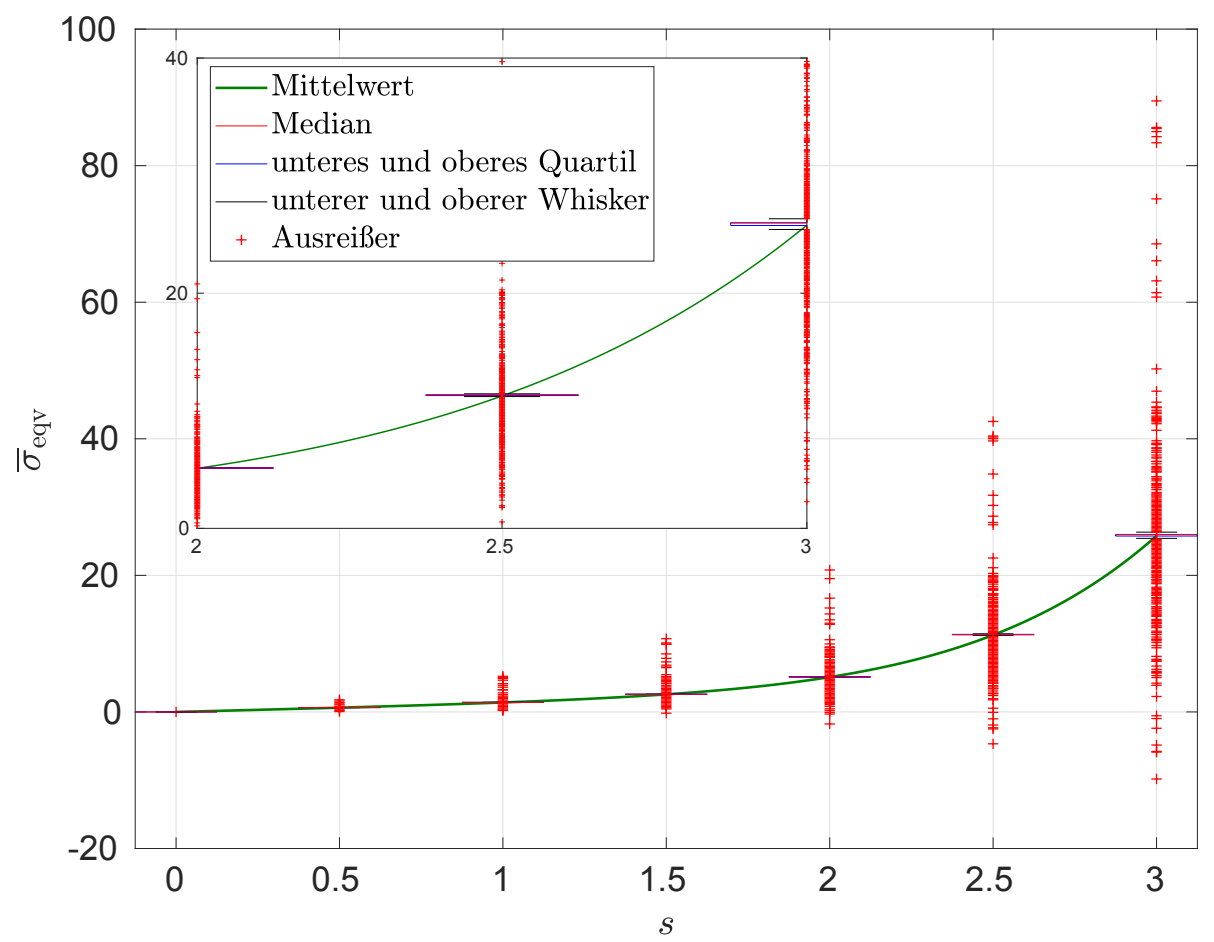

Abb. 4.6 - Mittelwert $\bar{\sigma}_{\text {eqv }}$ für den idealisierten Scherprobekörper (Simulationsergebnisse vgl. Tabelle 4.1 .

Sachverhalte deutlich, die im Folgenden näher diskutiert werden sollen. Zunächst wird ersichtlich, dass sich der Median (rote horizontale Linie) und der untere Whisker (2.5 \%Quantil) bzw. der obere Whisker (97.5\%-Quantil) sehr eng beieinander befinden. Dies bedeutet, dass sich $95 \%$ aller Messwerte sehr nah um den Mittelwert aufhalten. Lediglich die hier rot dargestellten Pluszeichen stellen Ausreißer dar, die aus den freien Rändern des idealisierten Scherprobekörpers resultieren. Da diese wenigen Ausreißer aber verhältnismäßig groß ausfallen (Abweichungen bis $250 \%$ ), haben sie einen starken Einfluss auf die Berechnung des Inhomogenitätsmaßes $\xi_{\mathrm{s}}$ (vgl. Gleichung (4-7)). Da mit Hilfe des Inhomogenitätsmaßes primär der Einfluss der verschiedenen Klemmdesigns auf die Homogenität untersucht werden soll, werden im Folgenden die freien Ränder bei der Auswertung nicht mit betrachtet. In der Abbildung 4.7 ist das Inhomogenitätsmaß $\xi_{\mathrm{s}}$ für den vollständigen $(10 \times 100 \times 2)$ und reduzierten Auswertebereich $(10 \times 80 \times 2)$ des idealisierten Scherprobekörpers dargestellt. Anhand der Verläufe wird qualitativ deutlich, wie stark sich die freien Ränder auf die Inhomogenitätsberechnung auswirken. Es sei nochmal darauf verwiesen, dass bei der globalen Kraftauswertung (vgl. Gleichung (4-6) ) die freien Ränder mit berücksichtigt werden und die daraus resultierenden Abweichungen nur gering ausfallen. 


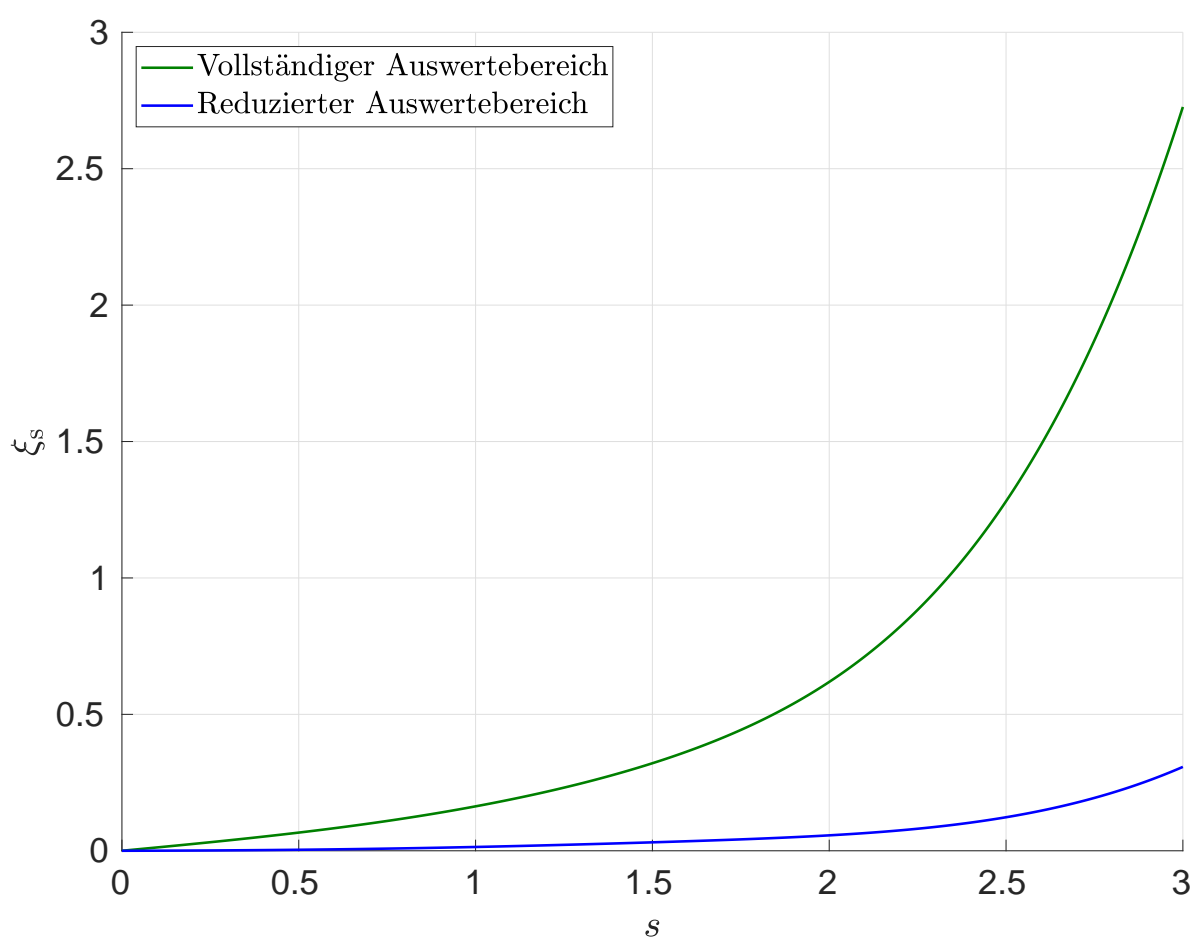

Abb. 4.7 - Inhomogenitätsmaß $\xi_{\text {s }}$ für den idealisierten Scherprobekörper für unterschiedliche Auswertebereiche (Simulationsergebnisse vgl. Tabelle 4.1 P

Für die Untersuchung von Beuleffekten an Schermatten soll in Anlehnung an Gleichung (3-48) ein modifiziertes Instabilitätsmaß $\kappa$ eingeführt werden:

$$
\kappa:=\left|\frac{u_{z, \max }-u_{z, \min }}{t_{0}}\right| .
$$

Dieses ist in der Weise definiert, dass die Differenz der maximalen zur minimalen zVerschiebung, dividiert durch die Probekörperdicke $t_{0}$ angegeben wird. Damit kann in einfacher Art und Weise überprüft werden, wann ein Beulen einsetzt. Für die Charakterisierung von Schermatten der Abmessung: $10 \times 100$ wird im Folgenden die Dicke $t_{0} \in[2,4] \mathrm{mm}$ und die Winkelimperfektion $\varphi \in[2.5,10]^{\circ}$ variiert (vgl. Skizze in Abbildung 4.8). Da die Variation der Winkelimperfektion nur zu verhältnismäßig geringen Abweichungen führt, soll der besseren Veranschaulichung halber nur die Winkelimperfektion von $\varphi=5^{\circ}$ dargestellt werden. In der Abbildung 4.8 ist das Instabilitätsmaß $\kappa$ für die verschiedenen Probekörperdicken dargestellt. Da das Auftreten von Beuleffekten im Messbereich zu einer extremen Spannungsinhomogenität führt, müsste das Inhomogenitätsmaß $\xi_{\mathrm{s}}$ in ähnlicher Weise wie das Instabilitätsmaß $\kappa$ den Beulpunkt voraussagen können. Bei genauerer Betrachtung der Abbildung 4.8 wird erkennbar, dass beide Maße den gleichen Beulpunkt vorhersagen. Die Ergebnisse machen deutlich, dass mit den aktuellen Probekörperabmessungen: $10 \times 100$ nur für die Schermattendicke von $4 \mathrm{~mm}$ ein Schermaß von $s=2$ erzielt werden kann. Eine Variation der hyperelastischen Stoffgesetzparameter führte zu der gleichen Beul-Charakteristik. Im Anhang A.3 ist exemplarisch das Beulverhalten für eine 


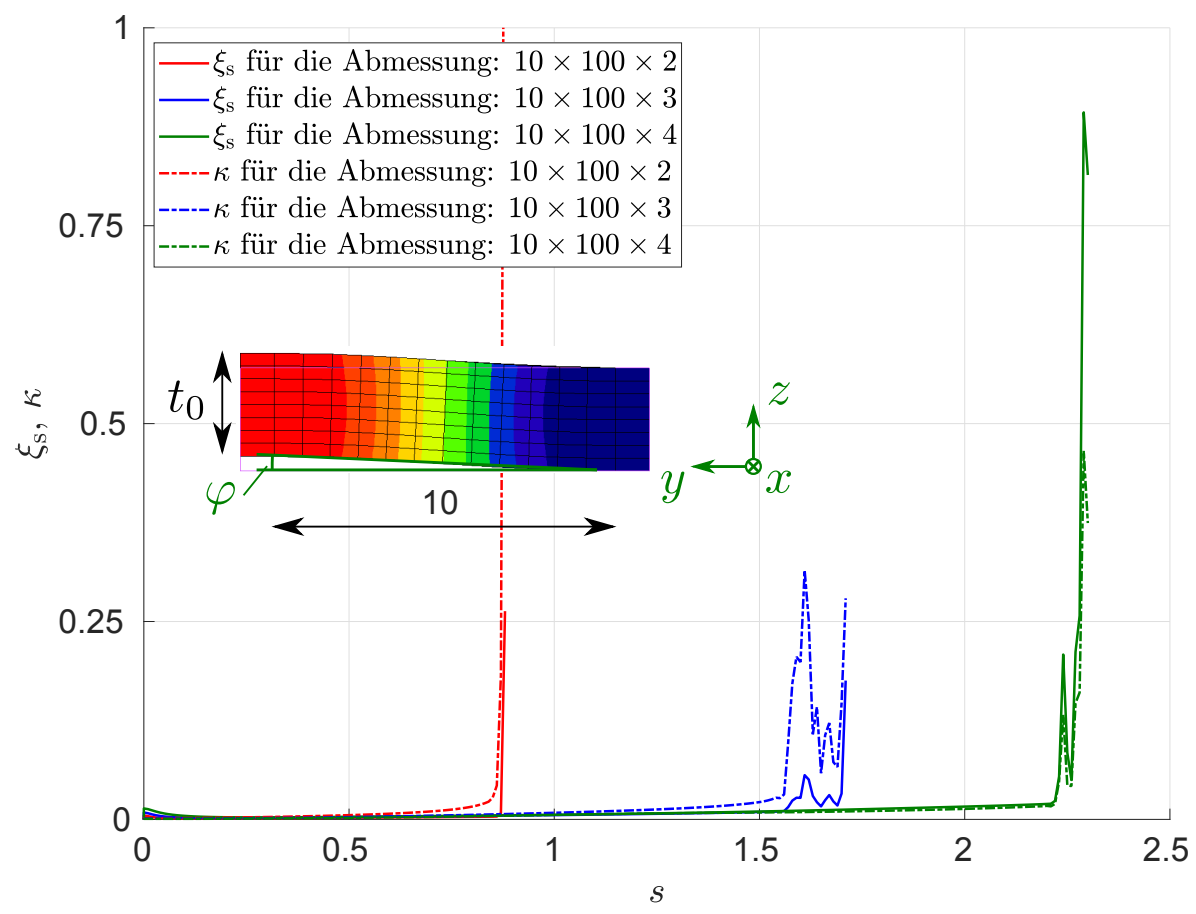

Abb. 4.8 - Beuluntersuchungen für den idealisierten Scherprobekörper unter Variation der Dicke $t_{0} \in[2,4] \mathrm{mm}$ bei einer Winkelimperfektion von $\varphi=5^{\circ}$ (Simulationsergebnisse vgl. Tabelle 4.1

Schermatte mit der Dicke $t_{0}=2 \mathrm{~mm}$ dargestellt. Es bleibt in nachfolgenden experimentellen Untersuchungen zu überprüfen, ob sich diese Beulpunkte bestätigen lassen.

\subsubsection{Untersuchung von Klemmdesigns}

Mit Hilfe der eingeführten Fehler- und Inhomogenitätsmaße sollen im Folgenden verschiedene Klemmdesigns getestet und qualitativ miteinander verglichen werden. Als Ausgangspunkt soll zunächst der Akzo Nobel Scherprobekörper (vgl. Abbildung 2.3) näher untersucht werden. Dieser Scherprobekörper wird standardmäßig über Klemmleisten verspannt und anschließend geschert. Um für spätere Vergleiche einheitliche Ausgangsgrößen zu haben, wird mit den Abmessungen: $10 \times 100 \times 2$ gerechnet. Aufbauend auf diesen Erkenntnissen sollen neue Designkonzepte entwickelt und simulativ getestet werden. In der Abbildung 4.9 ist das lokale Fehlermaß $\eta_{\mathrm{s}}$ für den Akzo Nobel Scherprobekörper für unterschiedliche Stauchungen durch die Klemmleisten dargestellt. Als Scherbelastung wurde eine Verschiebung von $u_{x}=10 \mathrm{~mm}$ vorgegebenen, die einem theoretischen Schermaß von $s=1$ entspricht. Für die Klemmleisten wurde ein Reibwert von $\mu=1$ angenommen. Anhand der Abbildung 4.9 wird deutlich, dass ohne eine Stauchung keine zufriedenstellende Scherdeformation eingeleitet werden kann. Der lokale Messfehler $\eta_{\mathrm{s}}$ im Zentrum des Auswertebereiches beträgt rund 0.5. Vergleicht man dazu den mittleren Bereich der sich bei einer Klemmbelastung von $5 \%$ bzw. $10 \%$ Stauchung einstellt, so wird deutlich, dass die Scherdeformation wesentlich besser übertragen werden kann. Der lokale Messfehler 


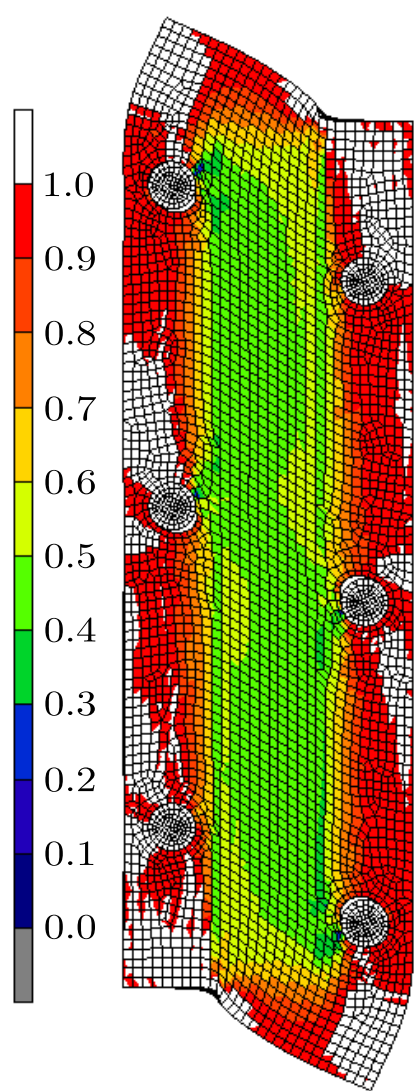

$0 \%$ Stauchung

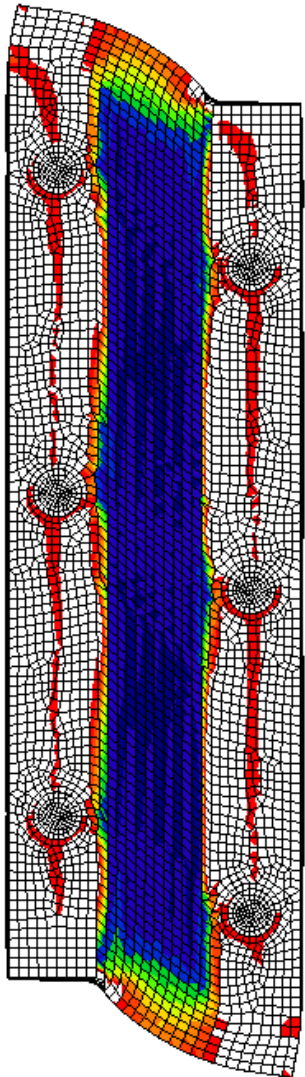

$5 \%$ Stauchung

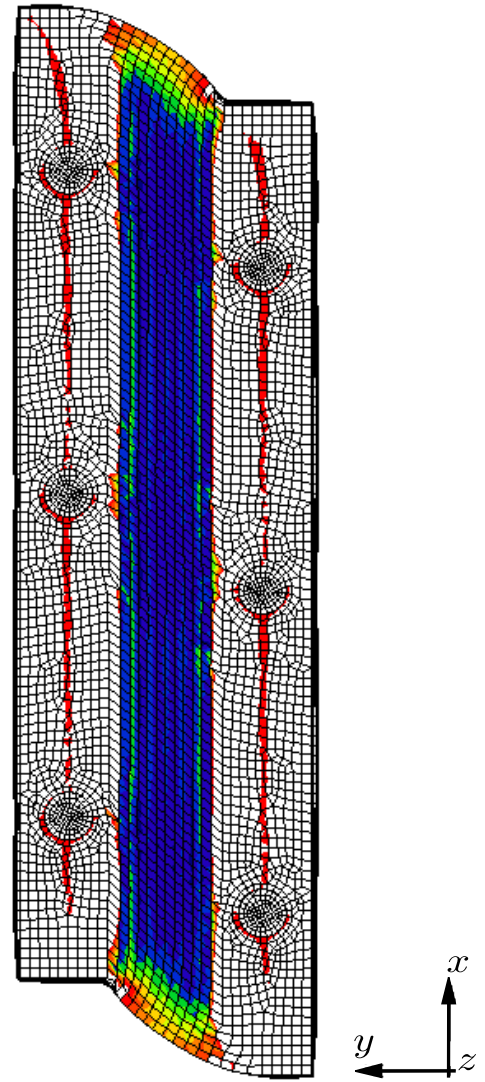

$10 \%$ Stauchung

Abb. 4.9 - Darstellung des lokalen Fehlermaßes $\eta_{\mathrm{s}}$ für den Akzo Nobel Scherprobekörper mit den Abmaßen: $10 \times 100 \times 2$ bei einer Scherbelastung von $u_{x}=10 \mathrm{~mm}$

der sich hier ergibt, liegt im Bereich von 0.2. Auffallend ist, dass sich im Zentrum des Messbereiches schwankende Fehlergrößen ausbilden.

Als nächstes soll das Spannungs-Schermaßdiagramm in Abbildung 4.10 und das sich daraus ergebende globale Messfehler-Schermaßdiagramm in Abbildung 4.11 näher betrachtet werden (bei einer Klemmbelastung von $5 \%$ Stauchung). Anhand der zweiten Abbildung wird deutlich, dass der globale Messfehler schon zu Beginn der Scherung eine Fehlergröße von ca. 0.25 aufweist. Damit liegt der Fehlerwert in der gleichen Größenordnung wie der eben beschriebene lokale Messfehler. Weiterhin wird ersichtlich, dass mit zunehmender Scherung ein monotoner Anstieg des globalen Messfehlers zu verzeichnen ist. Eine Erklärung kann darin gefunden werden, dass die Mattendicke (hier: $t_{0}=2 \mathrm{~mm}$ ) mit zunehmender Scherung leicht abnimmt, sich dadurch die Kontaktzonen verlagern und die Klemmleisten zwangsweise die Scherdeformation schlechter übertragen. Diese Überlegungen legen nahe, dass besonders der Einfluss der Randbedingungen in Tiefenrichtung ( $z$-Richtung) maßgebend für die Übertragung der Scherdeformation ist. Aus diesem Grund wird in einem nächsten Schritt der Deformationsverlauf über der Tiefe näher untersucht. Um weiterhin Einflüsse, die aus den freien Rändern resultieren zu umgehen, wird mit einem repräsentativen Mate- 


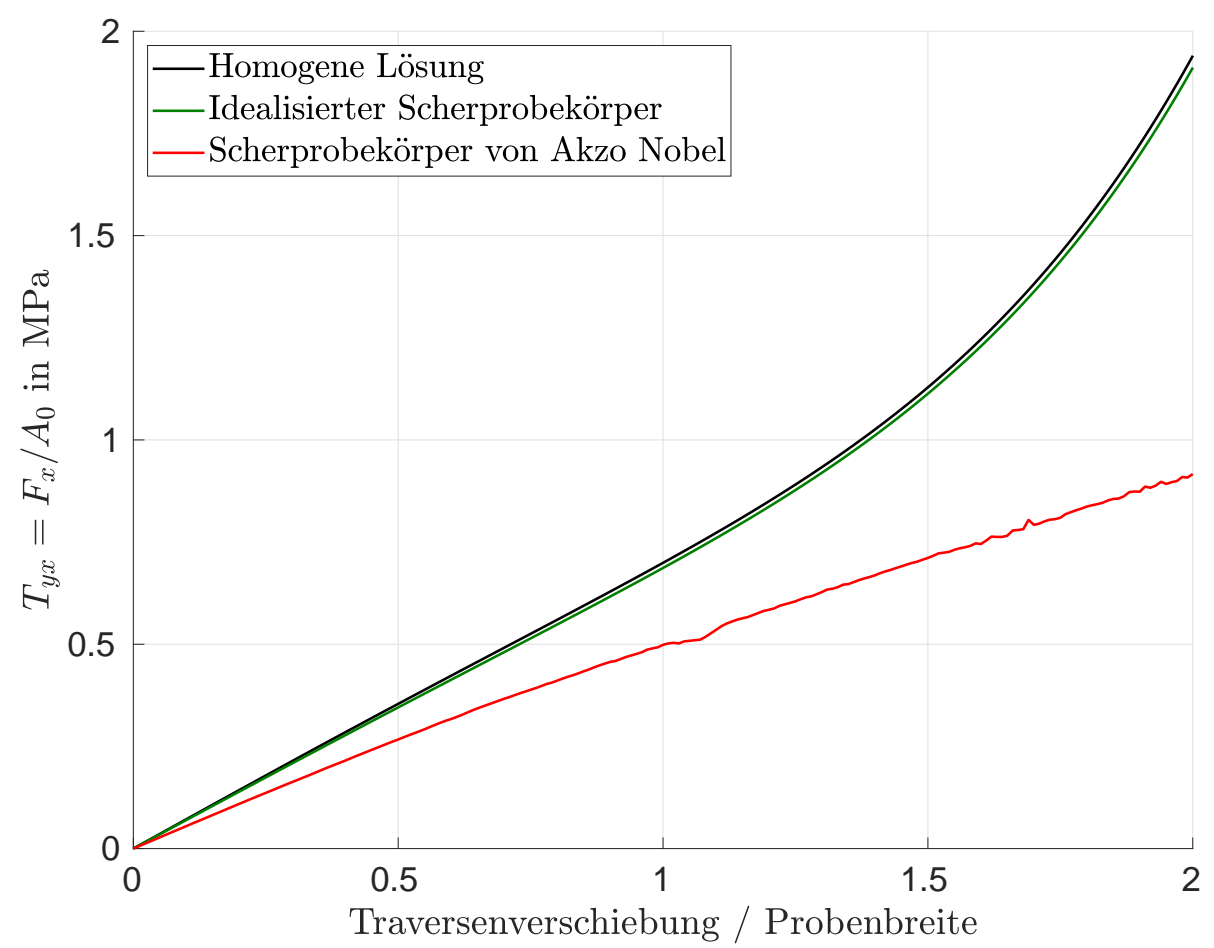

Abb. 4.10 - Spannungs-Schermaßdiagramm für den Akzo Nobel Scherprobekörper (Simulationsergebnisse vgl. Tabelle 4.1. vgl. Abbildung 4.4

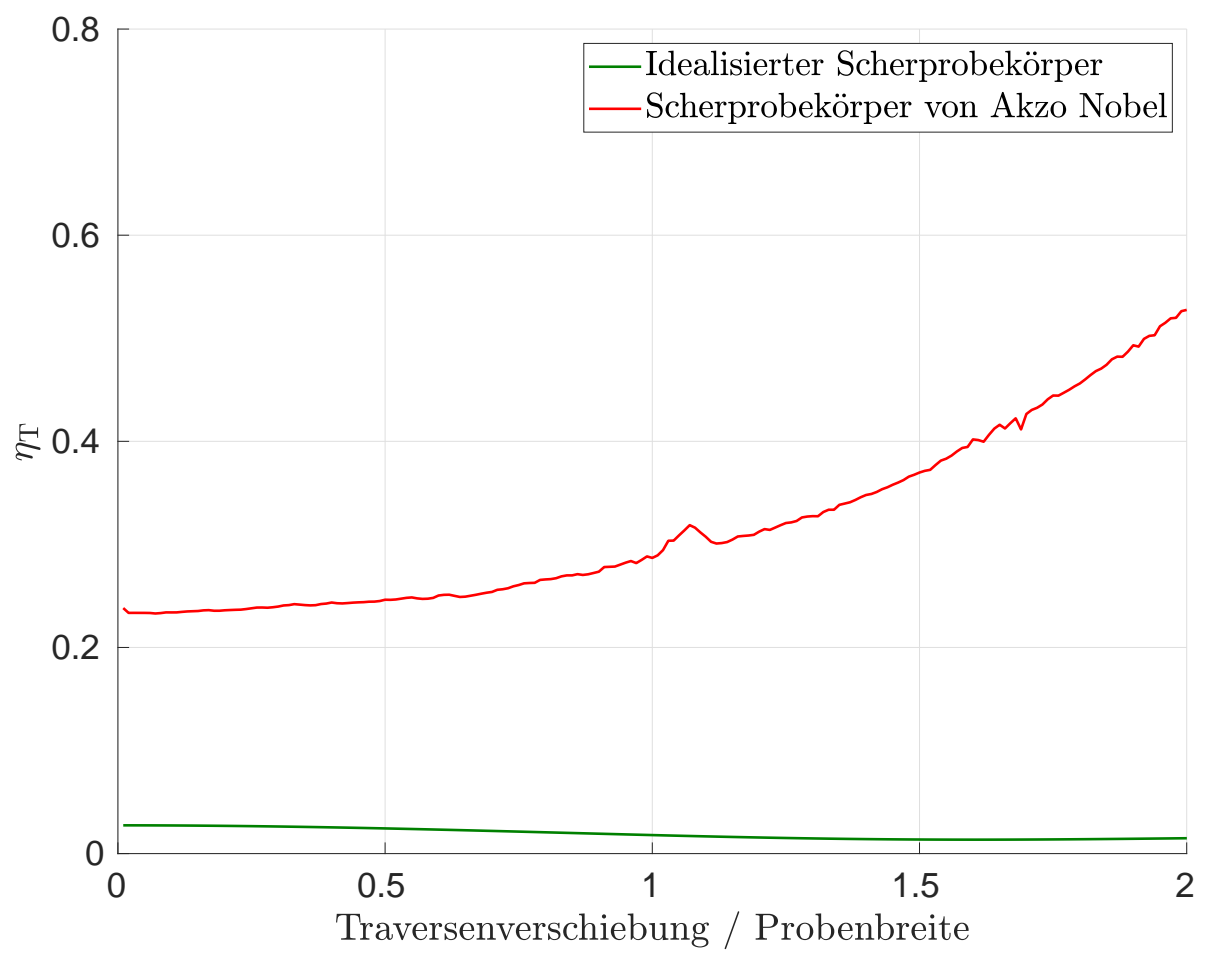

Abb. 4.11 - Globaler Messfehler $\eta_{\mathrm{T}}$ für den Akzo Nobel Scherprobekörper (Simulationsergebnisse vgl. Tabelle 4.1. vgl. Abbildung 4.5

rialstreifen mit periodischen Randbedingungen gearbeitet. Eine ausführliche Beschreibung 
der Besonderheiten von repräsentativen Volumenelementen (RVE) und der Erstellung von periodischen Randbedingungen (engl. periodic boundary conditions $=$ PBC) findet sich in der Arbeit von Donner (2017). In der Gleichung (4-9) ist der Zusammenhang der Ortsvektoren zwischen der Referenz- und der Momentankonfiguration dargestellt:

$$
\underline{r}^{k^{+}}-\underline{r}^{k^{-}}=\underline{\underline{F}} \cdot\left(\underline{\widetilde{r}}^{k^{+}}-\underline{\widetilde{r}}^{k^{-}}\right) \forall \underline{r} \in A^{k}
$$

Die Indizes $k^{+}$und $k^{-}$beschreiben dabei die zugeordneten Flächenpaare (vgl. auch Pahr \& Zysset 2008). In der Abbildung 4.12 ist ein repräsentativer Materialstreifen mit periodischen Randbedingungen in $x$-Richtung dargestellt. Dies bedeutet, dass man sich den

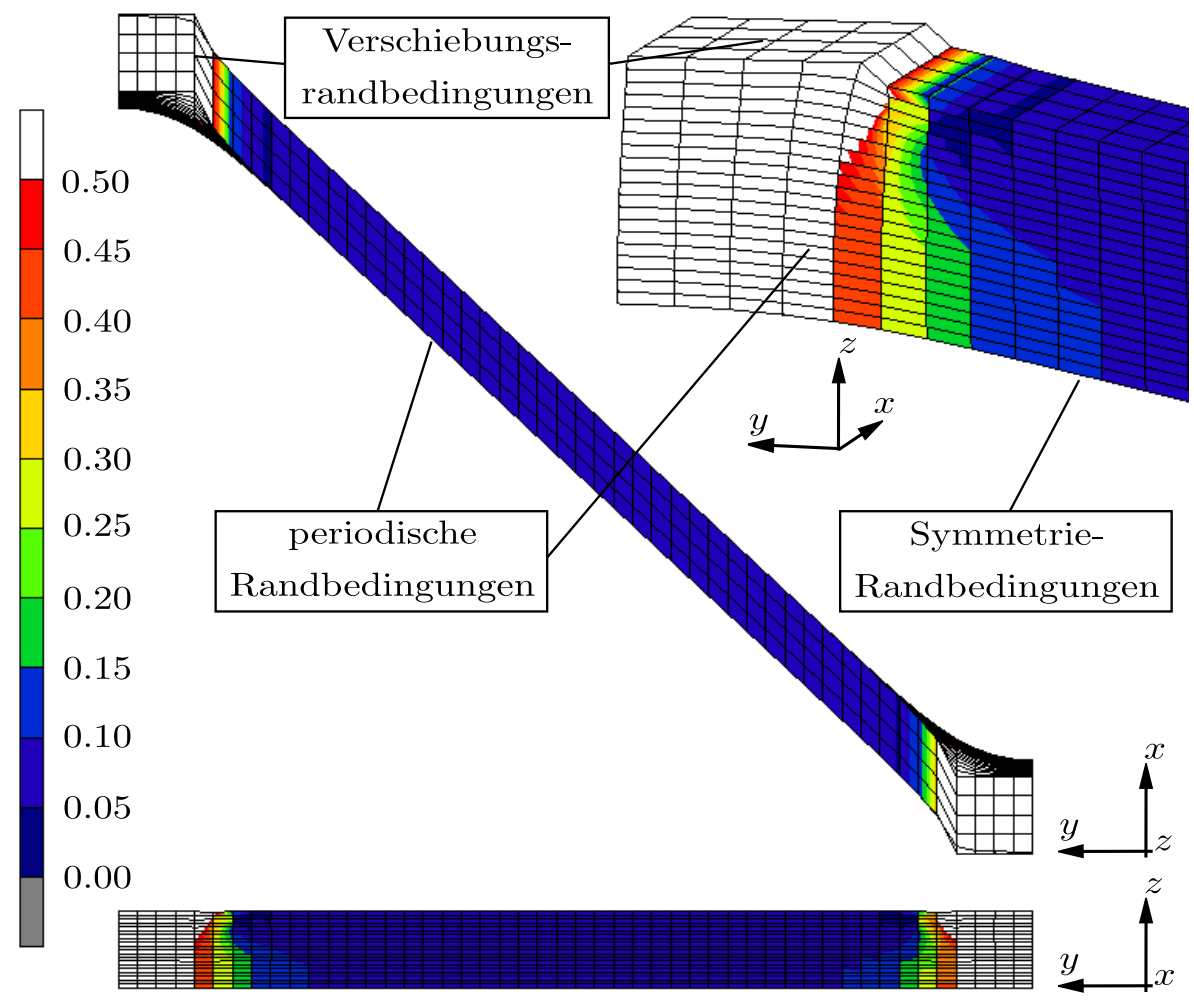

Abb. 4.12 - Darstellung des lokalen Fehlermaßes $\eta_{\mathrm{s}}$ an einem repräsentativen Materialstreifen mit fixierten Stirnflächen bei einem Schermaß von $s=1$

Materialstreifen in $x$-Richtung beliebig oft periodisch fortgesetzt vorstellen kann. Solche Randbedingungen besitzen den entscheidenden Vorteil, dass sich gegenüberliegende Flächen in gleicher Weise deformieren und dadurch Einflüsse, die Flächenverwölbungen hervorrufen, berücksichtigt werden können. Für den hier dargestellten Materialstreifen wird weiterhin in der $x y$-Ebene Symmetrie ausgenutzt. Die Einleitung der Scherdeformation erfolgt über Verschiebungsrandbedingungen, die an den Deckflächen des Materialstreifens aufgebracht werden (siehe quadratische Fläche in positiver $z$-Richtung). Anhand des oberen Ausschnittes in der Abbildung 4.12 wird deutlich, dass sich über der Tiefe ein inhomogenes Verzerrungsfeld einstellt. Weiterhin wird anhand der mittleren Darstellung deutlich, dass sich nahe des Einspannbereiches eine Verwölbung in $x$-Richtung ausbildet. 
Die wichtigsten Erkenntnisse aus den bisherigen Untersuchungen sollen kompakt zusammengetragen werden:

- Ein Kraftschluss der Außenbereiche führt zu keiner zufriedenstellenden Scherdeformation. Weiterhin wird Material aus dem Klemmbereich in den Auswertebereich gedrückt, was zu Spannungsinhomogenitäten führt.

- Verschiebungsrandbedingungen auf den Deckflächen des Materialstreifens führen zu einem inhomogenen Verzerrungsfeld über der Tiefe.

- Das sich dabei einstellende Inhomogenitätsprofil wird mit zunehmender Fixierung von Knoten in $z$-Richtung kleiner (numerisch getestet, aber nicht mit dargestellt).

- Idealerweise wäre ein Klemm- bzw. Spannmechanismus, der gleichmäßig über der Tiefe einsetzbar ist, wünschenswert.

\subsubsection{Numerische Entwicklung eines Scherprobekörpers mit formschlüssiger Lasteinleitung}

Aufbauend auf den bisherigen Erkenntnissen wird ein neues Designkonzept vorgestellt, welches eine gleichmäßige Einleitung der Scherdeformation über der Tiefe ermöglicht. Der Grundgedanke basiert dabei auf einer formschlüssigen Verbindung zwischen einer gleichmäßig gelochten Elastomermatte und dafür speziell vorgesehenen Stiften, welche die Matte führen. Durch diese Herangehensweise soll sichergestellt werden, dass der messtechnisch relevante Bereich homogen deformiert wird. In der Abbildung 4.13 ist zum besseren Verständnis eine Prinzipskizze dargestellt. Dieses Designkonzept bietet eine Reihe ganz prinzipieller Vorteile (aber auch Nachteile), die im Weiteren näher diskutiert werden sollen. Der große Vorteil dieses Designs besteht darin, dass die Scherdeformation sehr präzise über der gesamten Tiefe der Elastomermatte eingeleitet werden kann. Weiterhin wird, im Vergleich zu bisherigen mechanischen Klemmungen, kein Material in den Messbereich gedrückt bzw. verdrängt. Die Verwendung von Stiften hat auch den ganz praktischen Vorteil, dass auf eine stoffschlüssige Verbindung wie Kleben oder Anvulkanisieren verzichtet werden kann (vgl. Abschnitt 2.2.3). Gerade bei größeren Scherdeformationen $s>1$ kann es bei speziellen Kautschukmischungen dazu kommen, dass die Grenzschicht (bestehend aus Haftvermittler und Bindemittel) versagt und ein Einreißen stattfindet (vgl. Ballhorn 2007). Eine formschlüssige Verbindung könnte hier signifikante Vorteile aufweisen, vorausgesetzt, dass die Stifte nicht zu einem Einreißen der zuvor präparierten Elastomermatte führen. Als definitiven Nachteil kann aber angegeben werden, dass das neue Designkonzept in keiner Weise für Lebensdaueruntersuchungen geeignet ist. Da diese Anwendung aber kein vordergründiges Ziel darstellt, soll diese Einschränkung zunächst hingenommen werden.

Um das Potenzial dieses Designkonzepts abschätzen zu können, ist in der Abbildung 4.14 


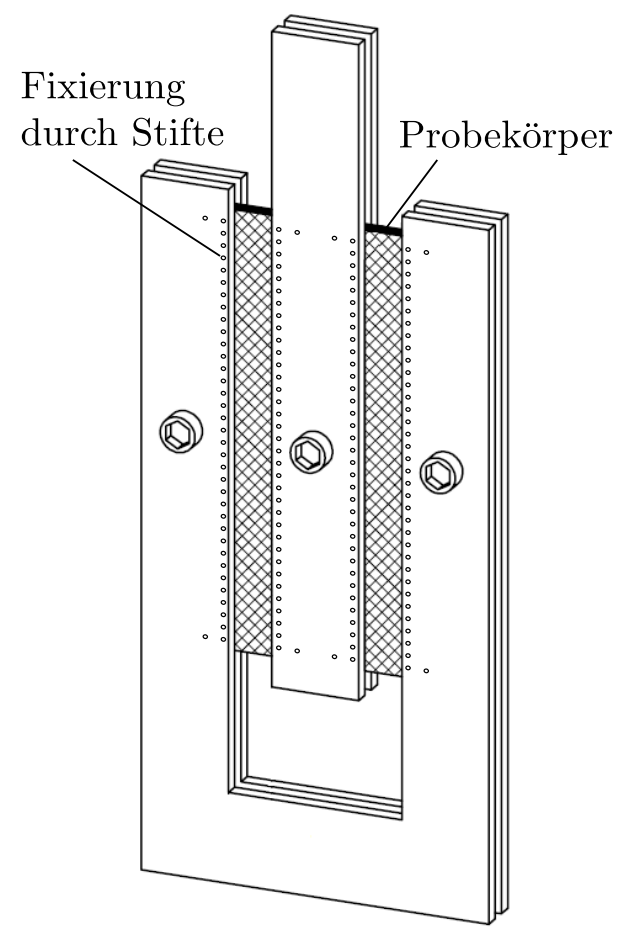

Abb. 4.13 - Prinzipskizze eines Scherprobekörpers mit formschlüssiger Verbindung mittels Stiften (Grundgeometrie in Anlehnung an den Scherprobekörper der Firma Akzo Nobel, siehe Ihlemann 2003)

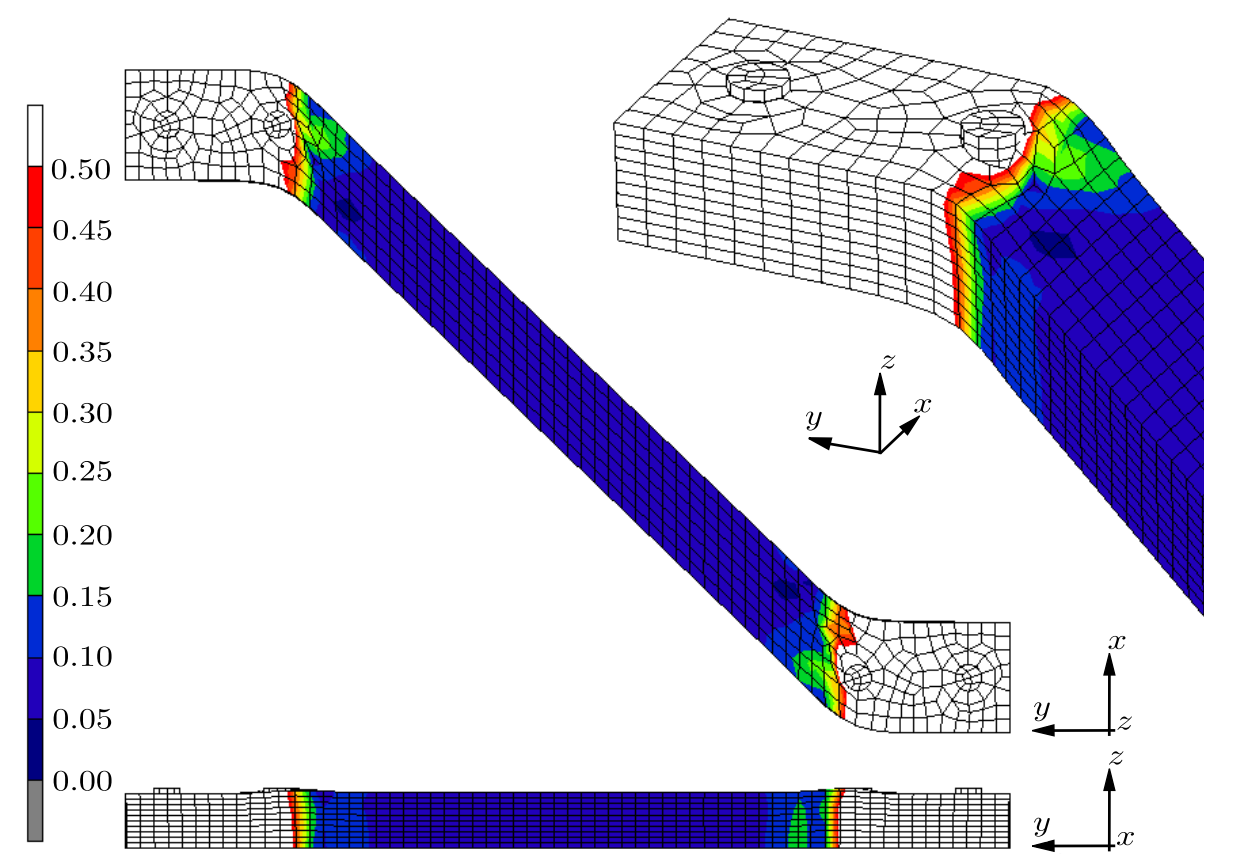

Abb. 4.14 - Darstellung des lokalen Fehlermaßes $\eta_{\mathrm{s}}$ für einen repräsentativen Materialstreifen (Pin-Durchmesser $d=0.5 \mathrm{~mm}$ ) bei einem Schermaß von $s=1$

ein repräsentativer Materialstreifen mit Stiften (im Weiteren auch als Pins bezeichnet) dargestellt. Bei der Modellierung des Materialstreifens mit periodischen Randbedingungen ist 
darauf zu achten, dass sich auf jeder Seite zwei Pins befinden müssen, um den Drehfreiheitsgrad um die $z$-Achse zu unterbinden. Anhand der Abbildung 4.14 werden folgende Sachverhalte deutlich. Zunächst ist zu erkennen, dass sich über der Tiefe ( $z$-Richtung) ein gleichmäßig deformierter Bereich einstellt. Im Vergleich zum Materialstreifen, der nur an den Deckflächen fixiert wurde, bleibt das sich hier ausbildende Verzerrungsfeld auch bei dickeren Elastomermatten erhalten. Weiterhin wird im direkten Vergleich mit dem Scherprobekörper von der Firma Akzo Nobel deutlich, dass das lokale Fehlermaß $\eta_{\mathrm{s}}$ im Messbereich wesentlich kleiner ausfällt.

Um nun auch globale Aussagen zum neuen Designkonzept treffen zu können, soll die Methode für eine Elastomermatte der Dimension: $10 \times 100 \times 2$ getestet werden. Dabei kommen zwei verschiedene Pin-Abmessungen und Stückzahlen zum Einsatz: $d_{\text {Pin }} \in[0.5,1] \mathrm{mm}$ und $n_{\text {Pin }} \in[25,50]$. Beide Parameterkonstellationen sollen zunächst eine erste Abschätzung zum Potenzial des neuen Designkonzepts angeben. In der Abbildung 4.15 ist der globale Messfehler $\eta_{\mathrm{T}}$ und in der Abbildung 4.16 das Inhomogenitätsmaß $\xi_{\mathrm{s}}$ dargestellt. Anhand

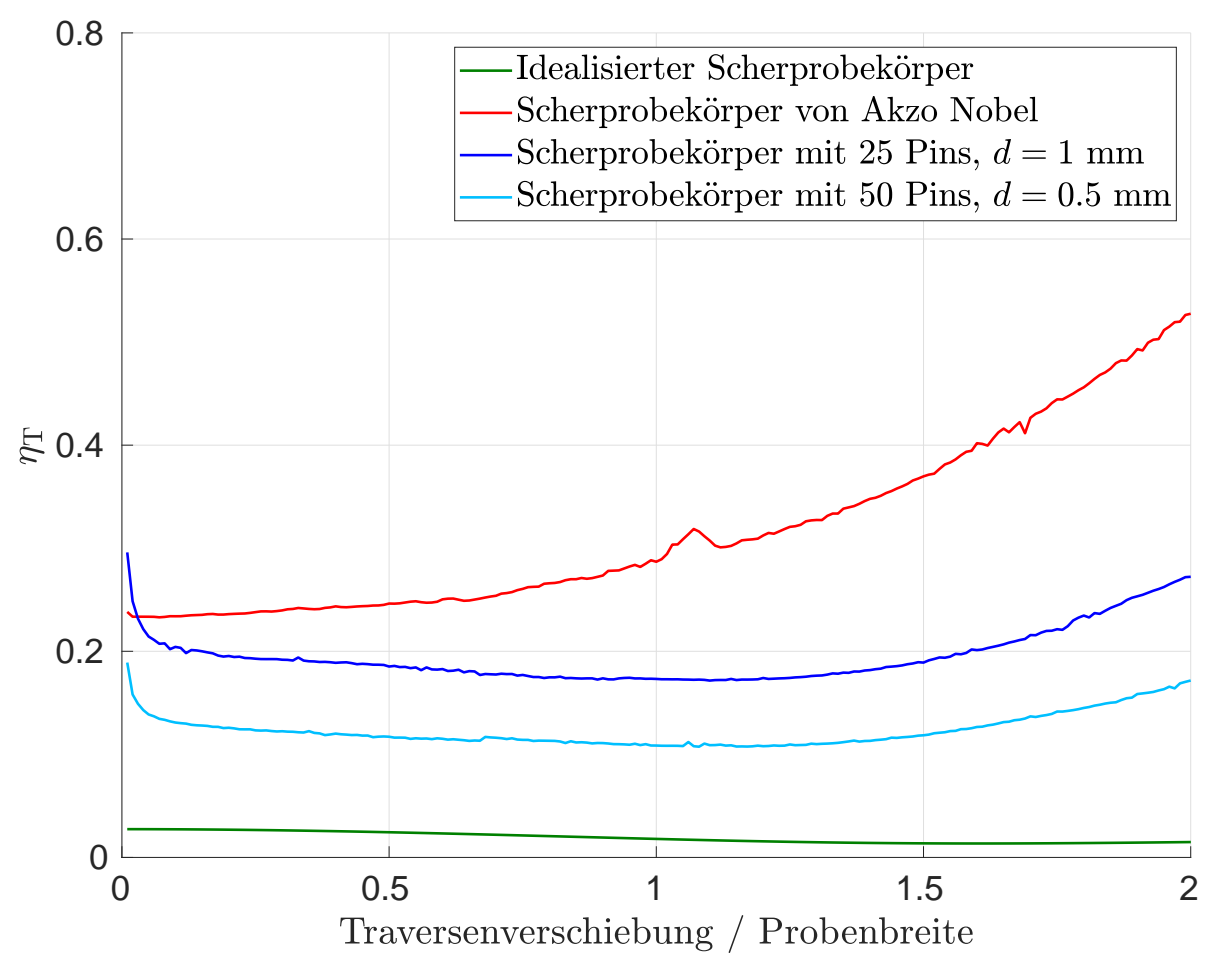

Abb. 4.15 - Darstellung des globalen Messfehlers $\eta_{\mathrm{T}}$ für verschiedene flächige Scherprobekörper (Simulationsergebnisse vgl. Tabelle 4.1. vgl. Abbildung 4.11

der Abbildung 4.15 wird deutlich, dass der globale Messfehler für die Pin-Varianten sehr viel geringer ausfällt als bei dem Scherprobekörper von der Firma Akzo Nobel. Dies zeigt sich insbesondere bei größeren Scherdeformationen (vgl. Traversenverschiebung/Probenbreite). Als nächstes soll das Inhomogenitätsdiagramm in Abbildung 4.16 näher betrachtet werden. Hier zeigt sich, dass das neue Designkonzept (bis Schermaße nahe eins) sehr geringe Abweichungen zum idealisierten Scherprobekörper aufweist. Erst bei größeren De- 


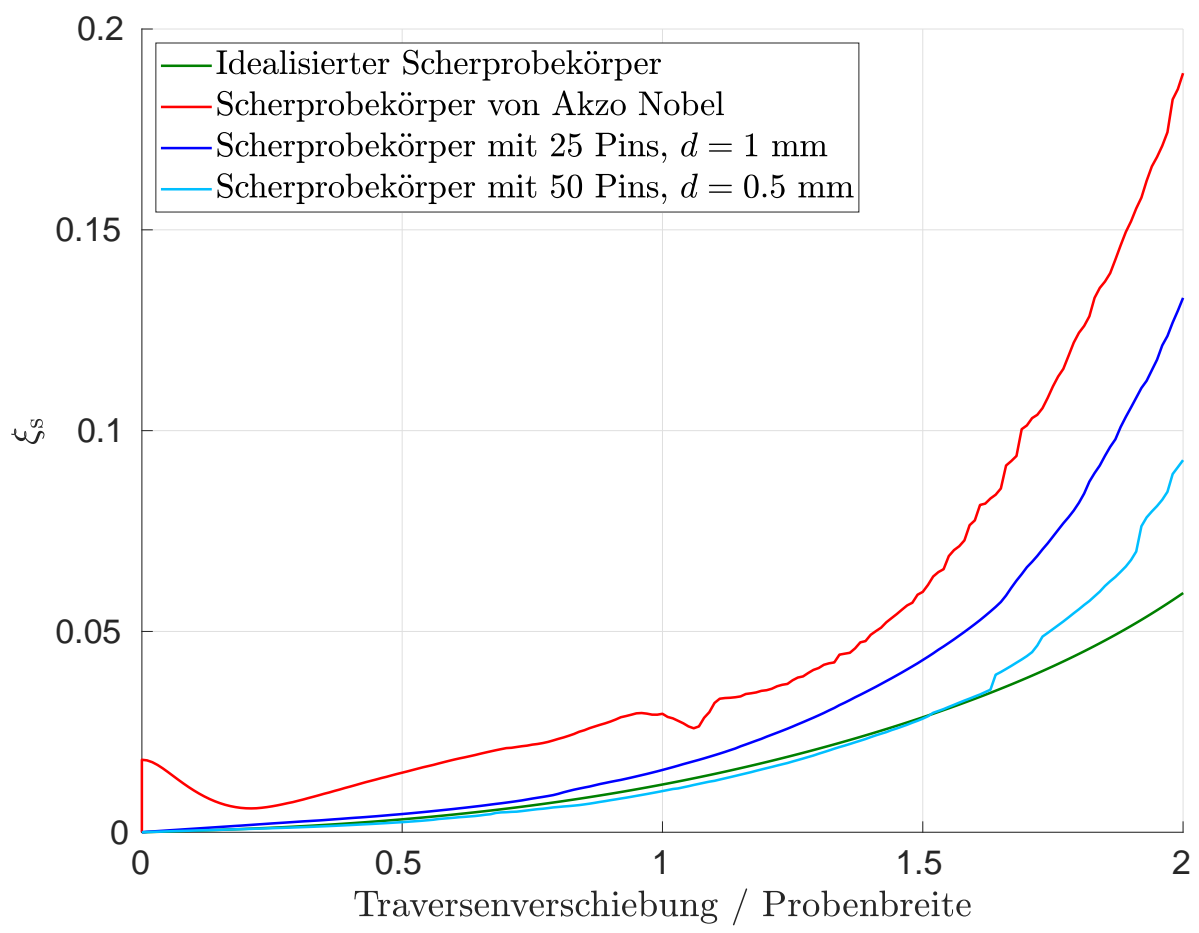

Abb. 4.16 - Darstellung des Inhomogenitätsmaßes $\xi_{\mathrm{s}}$ für verschiedene flächige Scherprobekörper (Simulationsergebnisse vgl. Tabelle 4.1. vgl. Abbildung 4.7)

formationen sind zunehmende Abweichungen zu verzeichnen. Ein direkter Vergleich mit dem Scherprobekörper von der Firma Akzo Nobel zeigt aber auch hier eine deutliche Verbesserung. Im Anhang ist in der Abbildung A.4 das lokale Fehlermaß $\eta_{\mathrm{s}}$ für den Scherprobekörper mit Pins dargestellt. Der Vollständigkeit halber sei an dieser Stelle erwähnt, dass andere entwickelte Einspann- bzw. Klemmvarianten mit unterschiedlichen Profilformen zu keiner wesentlichen Verbesserung der zu übertragenden Scherdeformation beigetragen haben. Aus diesem Grund wurde diesbezüglich auf eine weiterführende Vertiefung verzichtet.

\section{Zusammenfassung des neuen Designkonzepts:}

Das eingeführte Designkonzept, bestehend aus gelochter Elastomermatte und Pins zur Übertragung der Scherdeformation, zeigt eine deutliche Verbesserung gegenüber den herkömmlichen Klemmvarianten. Anhand numerischer Untersuchungen konnte gezeigt werden, dass die Pins zur formschlüssigen Lasteinleitung verwendet werden können. Diese Herangehensweise bringt den großen Vorteil mit sich, dass nun erstmalig flächige Elastomermatten nahezu homogen geschert werden können. Dies bietet insbesondere für Alterungsuntersuchungen, Versuche mit faserverstärkten Materialien und Versuche mit Vorreckung signifikante Vorteile. In experimentellen Untersuchungen bleibt zu klären, inwieweit das Designkonzept zu einem Einreißen der zuvor präparierten Elastomermatte führen kann. Eine weitere Herausforderung stellt die technologische Umsetzung der zu lochenden Matte und deren Monatge mit der Schervorrichtung über Stifte dar. Im nachfolgenden Abschnitt soll diesbezüglich eine erste experimentelle Umsetzung des Konzepts erfolgen. 


\subsection{Experimentelle Realisierung einer Schervorrichtung mit formschlüssiger Lasteinleitung}

\subsubsection{Versuchsaufbau der Schervorrichtung}

In diesem Abschnitt erfolgt die experimentelle Validierung des entwickelten Scherprobekörpers mittels Pins. Zunächst soll dazu näher auf den prinzipiellen Versuchsaufbau eingegangen werden. In der Abbildung 4.17 ist die Prüfmaschine mit eingebauter Schervorrichtung und 3D DIC-Messsystem dargestellt. Die Schervorrichtung lässt sich weiterhin in

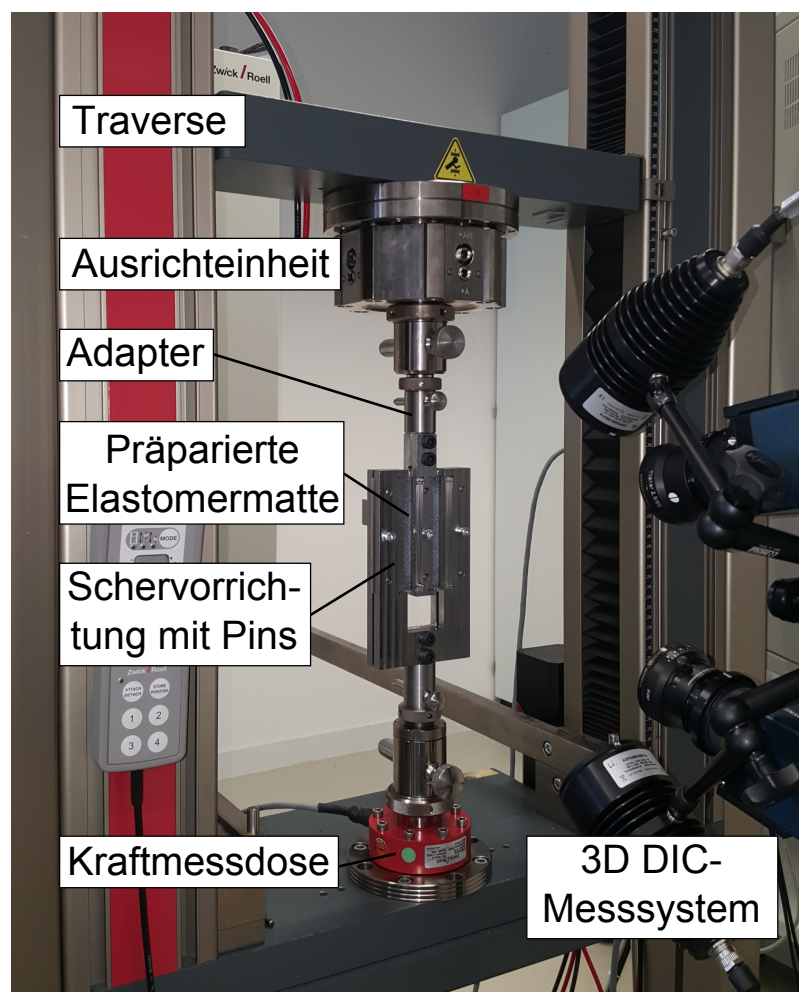

Abb. 4.17 - Versuchsaufbau der Schervorrichtung mit Messsystem

eine untere und eine obere Apparatur einteilen. Die untere Apparatur setzt sich dabei aus einem vorderen und einem hinterem U-Profil zusammen, welches für die starre Fixierung der Elastomermatte verantwortlich ist. Die obere Apparatur besteht ebenfalls aus einem vorderen und einem hinterem I-Profil, welches die verfahrbare Fixierung an der Traverse darstellt. Die präparierte Elastomermatte wird dabei lediglich über Pins mit der Schervorrichtung verbunden. Auf die spezielle Lochung der Elastomermatte soll im nächsten Abschnitt gesondert eingegangen werden. Um einen kraftfreien Einbau der Elastomermatte in die Prüfmaschine zu ermöglichen, kann auf dem hinteren U- und I-Profil eine Montageplatte befestigt werden. Diese gewährleistet beim Einbau eine starre Verbindung zwischen der unteren und der oberen Apparatur und ist insbesondere für sehr dünne Elastomermatten essenziell (vor der Messung zu entfernen). Die Schervorrichtung wird dabei in gleicher Weise wie das Probekörper-Setup über Adapter mit der Kraftmessdose (Nennlast: $20 \mathrm{kN}$ ) 
und der Ausrichteinheit verbunden. Letzteres spielt insbesondere bei der Korrektur von Positions- und Winkelabweichungen eine wichtige Rolle (vgl. dazu Abbildung 3.46). Die Verschiebungsmessung erfolgt auch hier optisch mittels eines 3D DIC-Messsystems (vgl. Abschnitt 3.4). Eine Besonderheit stellt hier lediglich der vertikale Versuchsaufbau des 3D DIC-Messsystems dar. Dieser ist darauf zurückzuführen, dass zum einen ein größerer Messbereich zur Verfügung steht, zum anderen eine bessere Ausleuchtung des Messbereiches möglich ist. Aufgrund der speziellen Geometrie der Schervorrichtung würde eine horizontale Beleuchtung zu einem kleineren detektierbaren Messbereich führen.

\subsubsection{Technologische Überlegungen zur Locherzeugung in Elastomermatten}

Im Folgenden soll auf die spezielle Lochung der Elastomermatte eingegangen werden. Wie in den numerischen Untersuchungen bereits gezeigt, wird die Scherdeformation mittels Pins über die Tiefe der Elastomermatte eingeleitet. Um die Vielzahl an Pins in die Elastomermatte einführen zu können, muss diese zuvor gelocht werden. Dies soll im Weiteren mit Hilfe spezieller Stanzwerkzeuge realisiert werden. In der Abbildung 4.18 ist die Matrix, in welche die einzelnen Stanzwerkzeuge eingesetzt werden können, dargestellt. Die

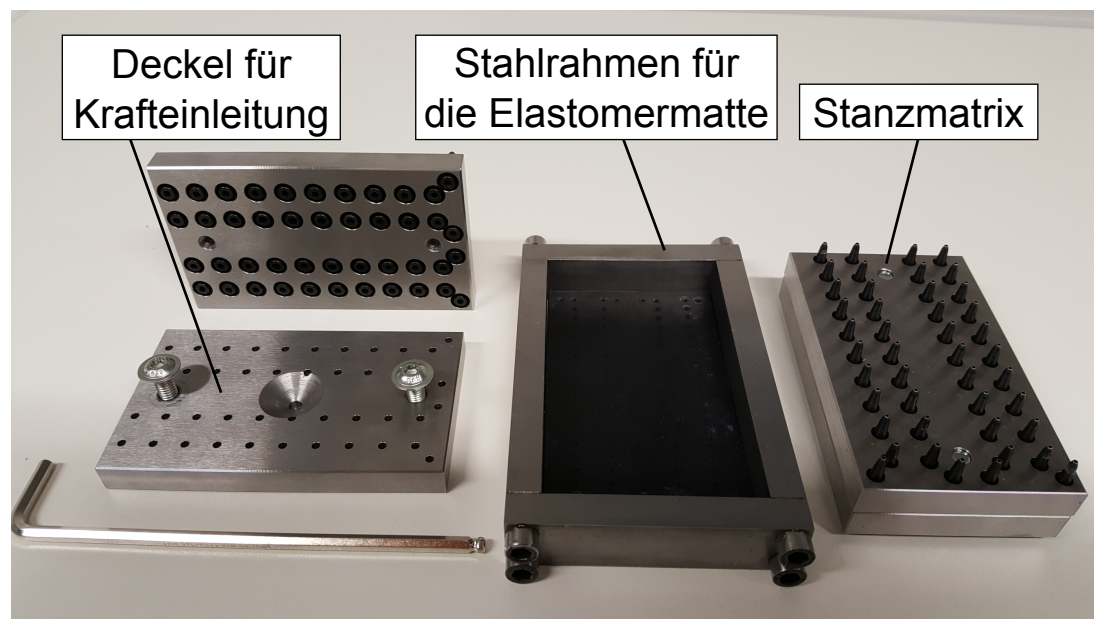

Abb. 4.18 - Lochung der Elastomermatte mittels Stanzmatrix

Matrix wurde dabei so konzipiert, dass verschiedene Stanzwerkzeuge mit unterschiedlichen Lochgrößen $d \in[1,2] \mathrm{mm}$ eingesetzt werden können. Eine weitere Besonderheit stellt das spezielle Muster der Bohrungen in der Matrix dar. Dieses wurde so erstellt, dass nach dem ersten Stanzvorgang die komplette Matrix um $180^{\circ}$ gedreht werden kann. Mittels eines Stahlrahmens, der als Führung dient, wird sichergestellt, dass alle Löcher symmetrisch gestanzt werden. Diese Herangehensweise bietet zwei prinzipielle Vorteile. Zum einen wird damit nur die Hälfte der Stanzkraft benötigt, was besonders für Mattendicken größer $6 \mathrm{~mm}$ empfehlenswert ist, zum anderen kann aufgrund der speziellen Geometrie der Stanzwerkzeuge die feine Reihung (hier Lochabstand $5 \mathrm{~mm}$ ) mit handelsüblichen Kaufteilen realisiert werden. Für den Deckel der Matrix wurde eine Kegelbohrung vorgesehen, damit durch eine 
Stahlkugel eine symmetrische Krafteinleitung erfolgen kann. Weiterhin befinden sich im Deckel kleine Durchgangsbohrungen, die einen Materialauswurf ermöglichen. Nach abgeschlossenem Stanzprozess wird die Elastomermatte für die DIC Messung beschichtet (vgl. Abbildung 3.49.

\subsubsection{Schermessungen mit Elastomermatten}

In diesem Abschnitt erfolgt die experimentelle Validierung der entwickelten Schervorrichtung mit der präparierten Elastomermatte (vgl. Abbildung 4.17). Um die Schervorrichtung auf ihre Funktionstauglichkeit testen zu können, wird zunächst ein zweiseitiger, gestufter Scherversuch durchgeführt. Dabei kann schrittweise überprüft werden, ob das Funktionsprinzip des Pin-Designs gewährleistet ist oder es zum Einreißen und damit frühzeitigen Versagen des Scherprobekörpers kommt. In Abbildung 4.19 ist der gestufte Versuch in einem Spannungs-Schermaßdiagramm für eine Matte aus gefülltem EPDM $\left(t_{0}=4 \mathrm{~mm}\right)$ dargestellt. Zunächst fällt der nahezu punktsymmetrische Spannungsverlauf auf, der für

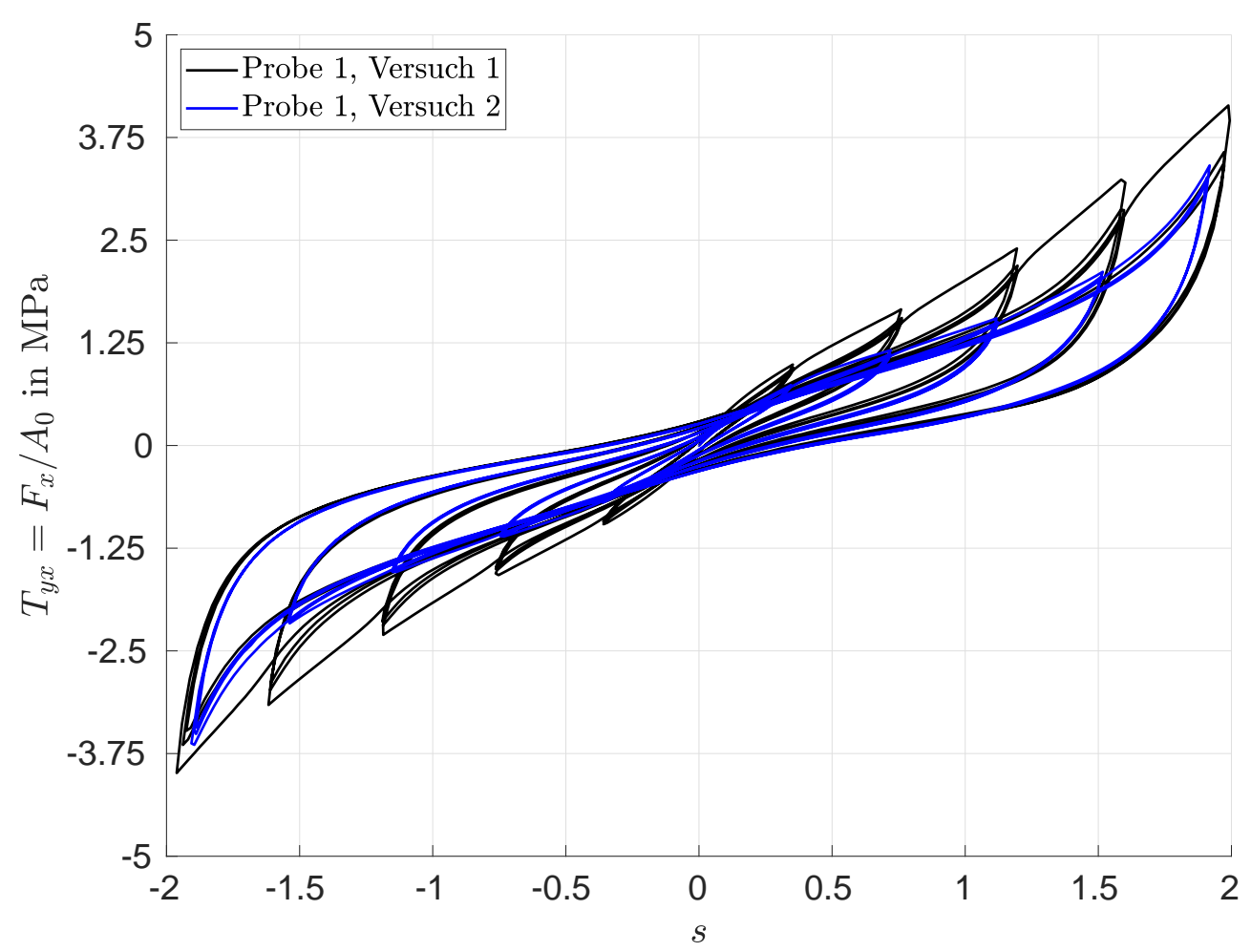

Abb. 4.19 - Symmetrischer Scherversuch mit der neuen Schervorrichtung (Messungen für gefüllten EPDM)

zweiseitig gestufte Scherversuche charakteristisch ist (vgl. Ahmadi u. a. 2007). Bei genauerer Betrachtung der Umkehrpunkte wird jedoch der Einfluss der belastungsinduzierten Anisotropie deutlich. Dieses Phänomen lässt sich dadurch erklären, dass bei der rechtsseitigen Scherung das Material erstmalig entfestigt wird, bevor die linksseitige Scherung erfolgt. Dadurch hat das Material bereits teilweise eine Entfestigung bei gleichem Schermaß erfahren, weshalb für die linksseitige Scherung weniger Kraft bei gleicher Deformation 
benötigt wird (vgl. auch Ihlemann 2003, Wulf 2016). Demgegenüber würde bei einer isotropen Entfestigung das Material nach der rechtsseitigen Scherung linksseitig komplett entfestigt sein. Weiterhin lassen sich aus der Abbildung 4.19 wichtige Eigenschaften wie Hysteresenform und bleibende Verzerrungen ableiten. Aus dem dargestellten SpannungsVerzerrungsdiagramm kann zunächst kein Einreißen oder frühzeitiges Versagen des PinDesigns festgestellt werden. Eine Demontage der Schervorrichtung bestätigt diesen Sachverhalt. Um die Schervorrichtung mit der Elastomermatte auf ihre Reproduzierbarkeit testen zu können, wird ein Wiederholungsversuch mit der gleichen Probe durchgeführt. Da die Probe bereits bis zu einem Schermaß von $s=2.0$ entfestigt wurde, müssten die stationären Zyklen der höchsten Laststufe aufeinanderliegen. Ein Vergleich zwischen Versuch 1 und 2 bestätigt diese Annahme. Damit kann sichergestellt werden, dass das Funktionsprinzip auch bei Wiederholungsversuchen gewährleistet ist. Anhand der Versuche 1 und 2 werden auch die verschiedenen Aspekte des Mullins-Effekt deutlich (Entfestigung durch Lastwiederholung und Entfestigung durch Vorreckung).

Als nächstes soll die Homogenität der Verzerrungen im Messbereich näher untersucht werden. Dafür erfolgt zunächst die Verschiebungsmessung optisch mittels eines 3D DICMesssystems. Um das Schermaß $s$ darzustellen, muss entweder auf die Schubverzerrung $\varepsilon_{x y}=s / 2.0$ oder den Scherwinkel $\varphi=\arctan (s)$ zurückgegriffen werden. Da in der Software von GOM Correlate die Schubverzerrungen aus dem jeweils lokal betrachteten Koordinatensystem berechnet werden und diese bei großen Schubverzerrungen stark vom Ausgangssystem abweichen können, wird der Scherwinkel $\varphi$ zur Schermaßberechnung herangezogen. In der Abbildung 4.20 sind die Messbereiche für zwei verschiedene Scherstufen

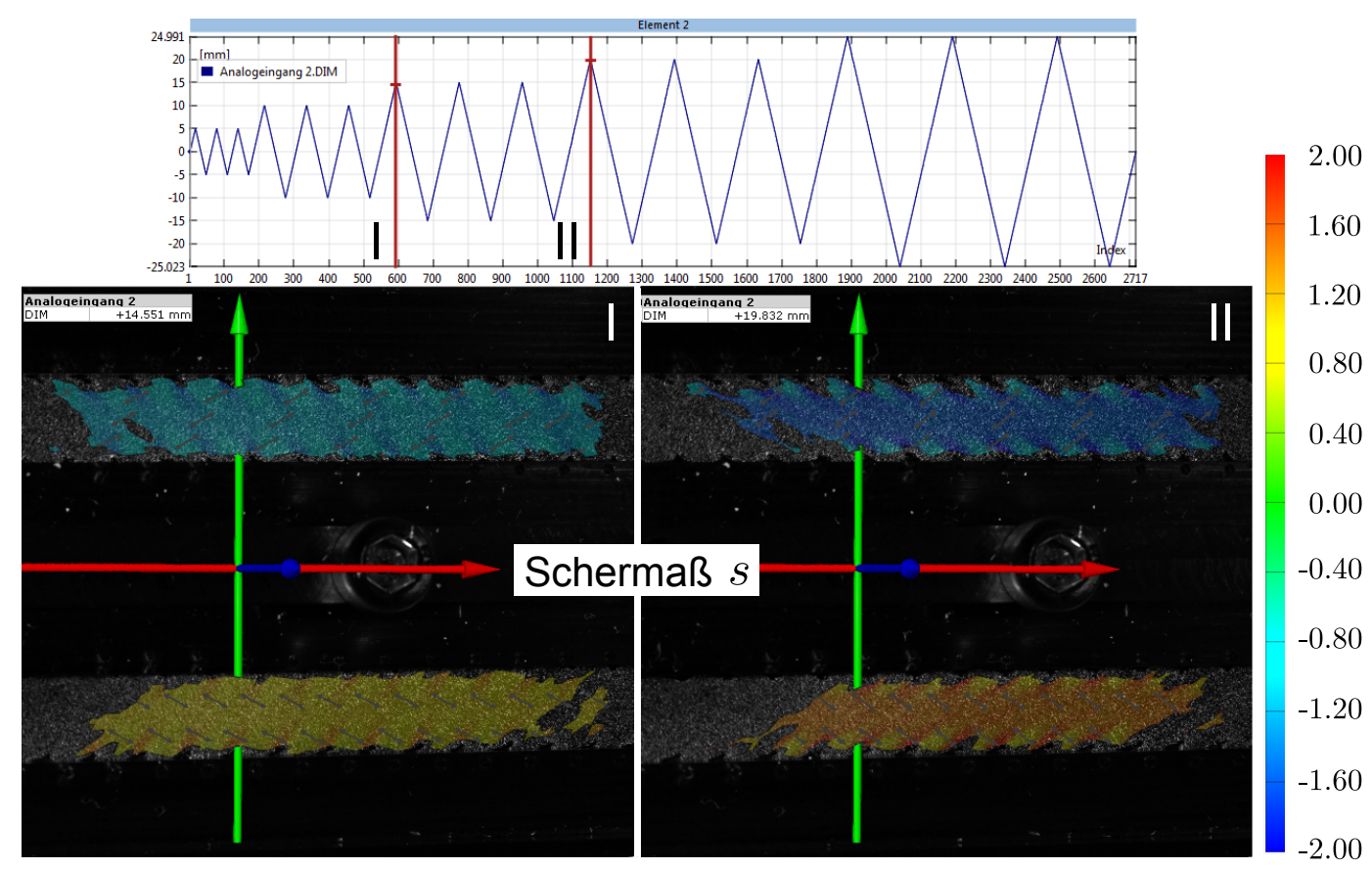

Abb. 4.20 - Lokale Darstellung des Schermaßes $s$ für verschiedene Laststufen 
dargestellt. Aus der linken Darstellung wird ersichtlich, dass sich bei einer Verschiebung von $u_{x}=15 \mathrm{~mm}$ (vgl. Diagramm in Abbildung 4.20) immer noch ein nahezu homogenes Verzerrungsfeld einstellt. Lediglich im Übergangsbereich zwischen gestanztem Loch und Pin sind größer werdende Inhomogenitäten der Verzerrung zu verzeichnen. Im Vergleich dazu sind die Inhomogenitäten in der rechten Darstellung (Verschiebung $u_{x}=20 \mathrm{~mm}$ ) im Übergangsbereich stärker ausgeprägt. Hier sollte insbesondere für eine globale Auswertung darauf geachtet werden, dass im Zentrum des Messbereiches gemessen wird. Nichtsdestotrotz zeigt der Scherprobekörper bei diesen großen Scherdeformationen (vgl. Abbildung 4.19 bei einem Schermaß von $s=1.6$ ) eine verhältnismäßig homogene Verzerrungsverteilung und bietet somit Potential für Weiterentwicklungen. Diesbezüglich sei darauf hingewiesen, dass diese Schervorrichtung ein erster Prototyp ist und zunächst die Funktionsweise des Pin-Konzepts sicherstellen soll. In weiterführenden Arbeiten wäre es überaus empfehlenswert sowohl den Pin-Durchmesser, als auch die Pin-Anzahl zur Verbesserung der Homogenität zu optimieren.

\section{Zusammenfassung der präzisen Schermessungen:}

Generell kann festgehalten werden, dass sich das Pin-Design für präzise Schermessungen eignet und sich dadurch eine Reihe interessanter Anwendungsmöglichkeiten wie Alterungsuntersuchungen mit Matten, Versuche mit faserverstärkten Materialien und Versuche mit Vorreckung auf Zug eröffnen. Weiterhin bietet eine automatisierte Stanz-/Fixiervorrichtung die Möglichkeit einer standardisierten Implementierung in den Industriealltag, bei der gänzlich auf aufwändige Vorarbeiten wie Kleben oder Anvulkaniseren verzichtet werden kann.

\section{Beuluntersuchungen}

Abschließend wird das Beulverhalten für verschiedene Mattendicken charakterisiert. In Abschnitt 4.4 .1 wurden dazu bereits erste Simulationsergebnisse gezeigt (vgl. Abbildung 4.8). Diese numerischen Untersuchungen sollen im Folgenden durch experimentelle Daten validiert werden. Hierfür werden Elastomermatten unterschiedlicher Dicke präpariert und in die Schervorrichtung eingebaut. Die Scherversuche werden dabei so realisiert, dass solange in eine Richtung geschert wird, bis ein eindeutiges Beulen zu verzeichnen ist. Im Anschluss daran wird auf Verschiebung Null zurückgefahren. In der Abbildung 4.21 ist das Spannungs-Schermaßdiagramm für drei verschiedene Mattendicken dargestellt. Dabei kann der grüne Verlauf (Mattendicke $t_{0}=4.4 \mathrm{~mm}$ ) bis zu einem Schermaß nahe $s=3.0$ als Referenzkurve angesehen werden. Die Abweichung der anderen beiden Verläufe (Mattendicke $t_{0}=2.3 \mathrm{~mm}$ und $t_{0}=3.4 \mathrm{~mm}$ ) zu dieser Referenzkurve kennzeichnet dabei den vermuteten Beulpunkt. Demzufolge scheint die Elastomermatte mit der Dicke $t_{0}=2.3 \mathrm{~mm}$ bei einem Schermaß von $s=1.1 \mathrm{zu}$ beulen und die Elastomermatte mit der Dicke $t_{0}=3.4 \mathrm{~mm}$ bei einem Schermaß von $s=2.0$. Für die Mattendicke $t_{0}=4.4 \mathrm{~mm}$ kann der Beulpunkt zunächst nur nahe dem Schermaß $s=3.0$ abgeschätzt werden. Um 


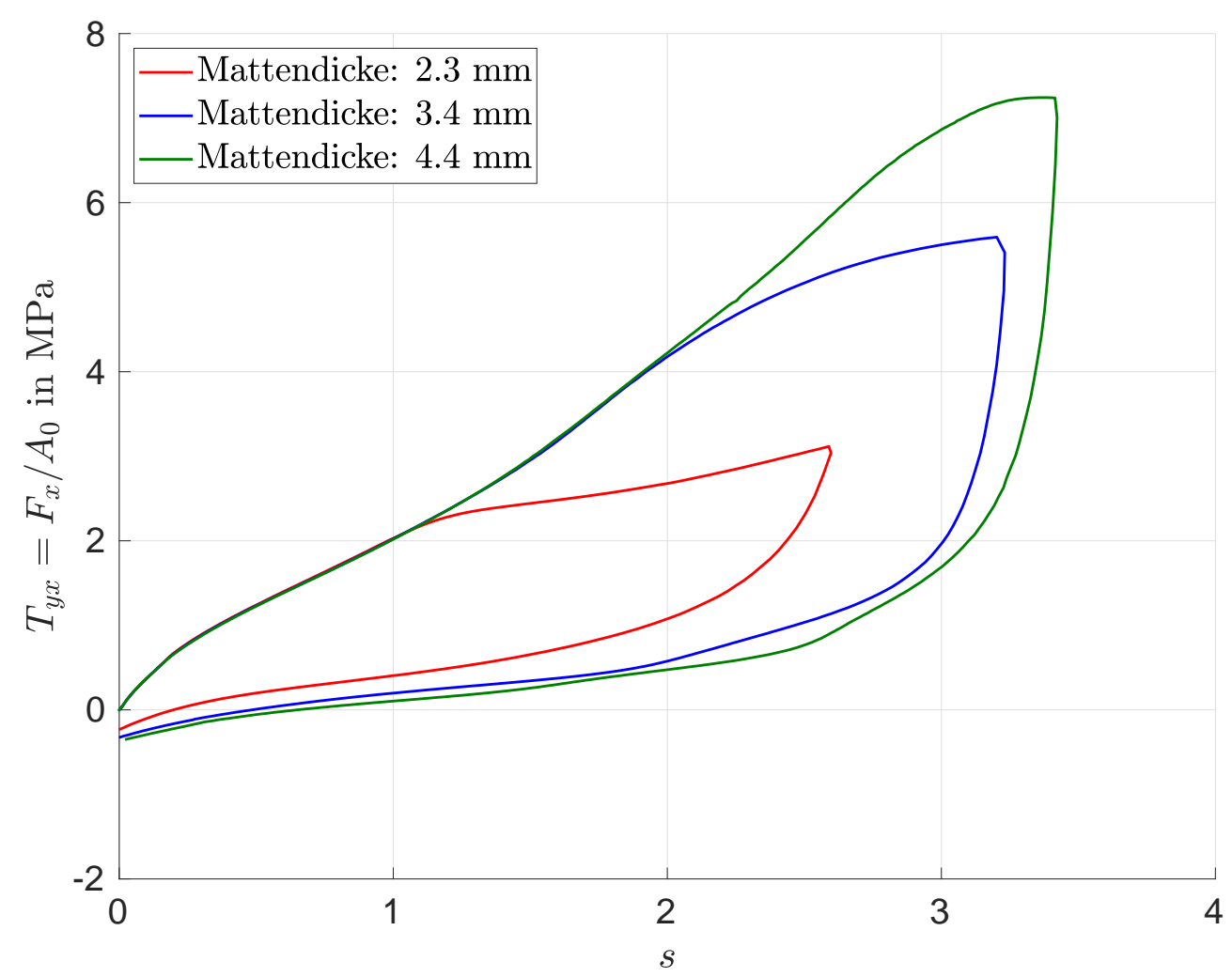

Abb. 4.21 - Spannungs-Schermaßdiagramm für verschiedene Mattendicken (Messungen für gefüllten EPDM)

diese Annahmen zu überprüfen, soll im Weiteren für die hier dargestellten Elastomermatten das Instabilitätsmaß $\kappa$ berechnet werden. Dies soll mit Hilfe der gemessenen „out-ofplane“-Komponente eines 3D DIC-Messsystems realisiert werden. In der Abbildung 4.22 ist das berechnete Instabilitätsmaß für die drei verschiedenen Mattendicken dargestellt. Zur besseren Vergleichbarkeit wurden ebenso die aus der Simulation berechneten Instabilitätsmaße mit abgebildet. Für den roten Verlauf der Mattendicke $t_{0}=2.3 \mathrm{~mm}$ ergibt sich ein signifikanter Anstieg des Instabilitätsmaßes bei $s=0.9$. Damit stimmt die Messung mit den simulierten Ergebnissen sehr gut überein. Für die Elastomermatte mit der Dicke $t_{0}=3.4 \mathrm{~mm}$ ist ein signifikanter Anstieg ab $s=1.6 \mathrm{zu}$ detektieren. Auch dieses Ergebnis kann gut mit den Vorhersagen aus der Simulation bestätigt werden. Als letztes soll der Verlauf der Mattendicke $t_{0}=4.4 \mathrm{~mm}$ näher betrachtet werden. Hier wird deutlich, dass bei einem Schermaß ab $s=2.6$ ein monoton steigender Verlauf des Instabilitätsmaßes zu verzeichnen ist. Dieses Ergebnis zeigt die größte Abweichung zu den simulierten Vorhersagen, welche den Beulpunkt bei $s=2.25$ angeben. Eine Erklärung kann darin gefunden werden, dass die hier verwendeten Elastomermatten herstellungsbedingt ein leichtes Übermaß aufweisen, welches sich im Bereich weniger Zehntel Millimeter bewegt. Dies würde auch erklären, warum die simulierten Daten eine leichte Unterschätzung der Beulpunkte angeben. Zusammenfassend kann aber festgehalten werden, dass sich die Beulpunkte in erster Näherung gut mit hyperelastischen Stoffgesetzen vorhersagen lassen. 


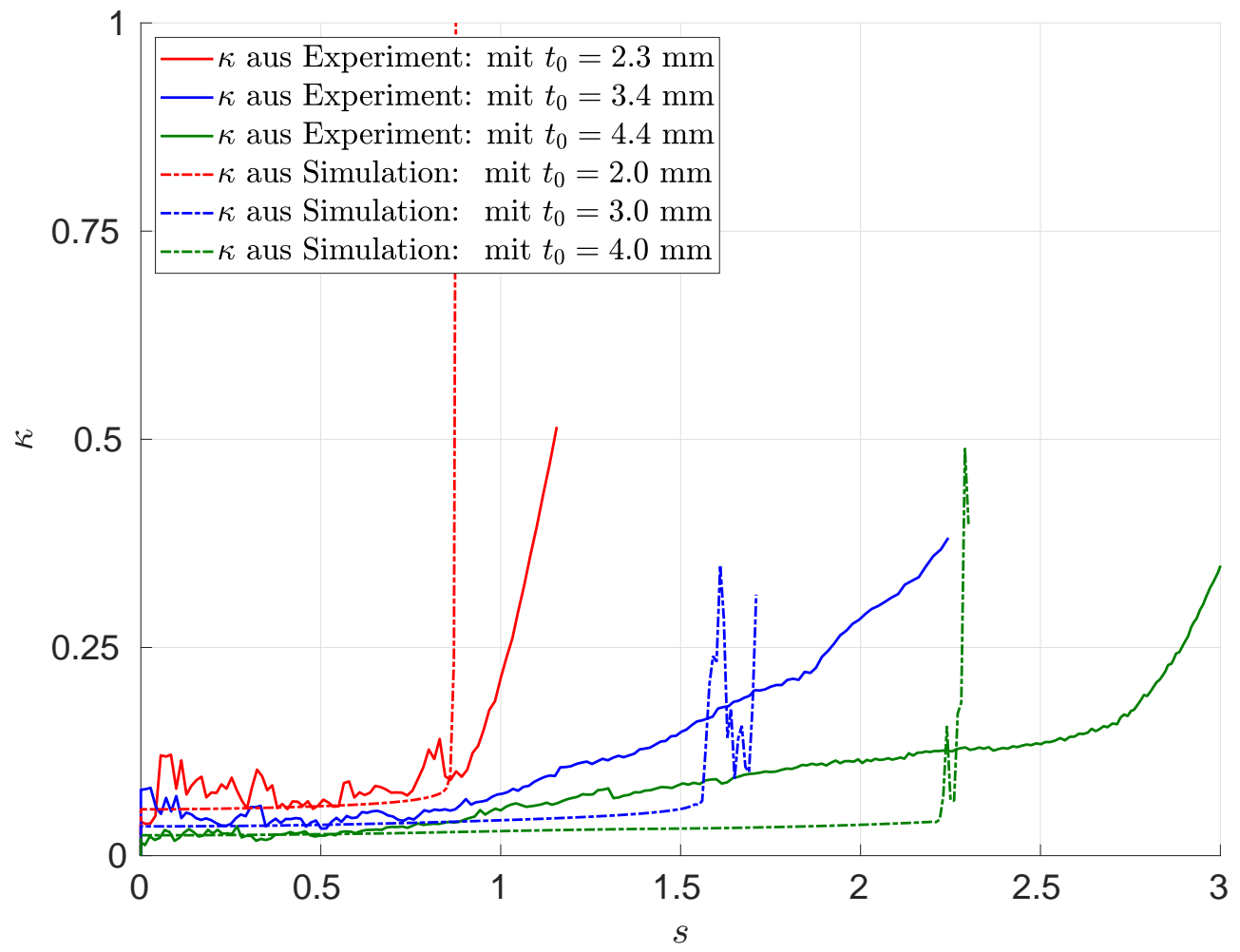

Abb. 4.22 - Kappa-Schermaßdiagramm für verschiedene Mattendicken (Simulationsergebnisse vgl. Tabelle 4.1 und Messungen für gefüllten EPDM, vgl. Abbildung 4.8 



\section{Anwendungsbeispiele für die entwickelten homogenen Probekörper}

In diesem Kapitel werden mit Hilfe der entwickelten homogenen Probekörper einige ausgewählte Anwendungsbeispiele vorgestellt. Nach einer Parameteranpassung eines viskoelastoplastischen Stoffgesetzes, vorzugsweise bei großen Stauchungen, kann eine Probekörpersimulation unter Berücksichtigung inelastischer Effekte durchgeführt werden. Als nächstes Anwendungsbeispiel soll das ratenabhängige Materialverhalten im Zug-/Druckbereich mit Hilfe ausgewählter Funktionssignale untersucht werden. Abschließend wird das ProbekörperSetup mittels einer automatischen Einspannvorrichtung für den Industriealltag zugänglich gemacht.

\subsection{Homogene Standardversuche zur Identifikation von Materialparametern}

Homogene Standardversuche werden sowohl für die phänomenologische Untersuchung technischer Elastomere als auch für die Parameteranpassung von Stoffgesetzen benötigt. In diesem Abschnitt soll zunächst ein viskoelastoplastisches Stoffgesetz, welches die komplexe Phänomenologie von technischen Elastomeren sehr gut beschreibt, vorgestellt werden. Im Anschluss daran erfolgt eine Parameteranpassung anhand der homogenen Zug/Druckversuche. Durch den Vergleich einer homogenen Simulation (Simulation mit einem finiten Element) mit einer Probekörpersimulation kann in direkter Weise das große Potential des Probekörper-Setups aufgezeigt werden. Dadurch wird auch einmal mehr ersichtlich, wie eng die Stoffgesetzentwicklung mit der experimentellen Versuchsdurchführung verbunden ist. Abschließend wird das im Experiment gemessene globale Kraft-Wegverhalten mit der Vorhersage einer Probekörpersimulation verglichen.

Im Folgenden soll das viskoelastoplastische Stoffgesetz, welches in Kooperation mit der Firma Vibracoustic GmbH unter den Vorarbeiten von Michael Rabkin entwickelt wurde, sehr kompakt vorgestellt werden (nachfolgend als Rabkin-Modell bezeichnet). Das Stoffgesetz gliedert sich dabei phänomenologisch in drei Spannungsanteile auf, die additiv miteinander verrechnet werden. In der Gleichung (5-1) sind die verschiedenen Spannungsanteile als hyperelastisch, elastoplastisch und viskoelastisch gekennzeichnet:

$$
T=T_{\text {he }}+T_{\text {ep }}+T_{\text {ve }} .
$$


Zur Beschreibung des hyperelastischen Anteils wird einer Gleichgewichtsfeder das nichtaffine Röhrenmodell von Heinrich u. a. (1988) zugrunde gelegt. Bei diesem Modell erfolgt eine Berücksichtigung der Behinderung von Netzwerkketten durch die Verschlaufung mit benachbarten Ketten. Der Begriff „nicht-affine Röhre“ ist dabei auf die topologische Behinderung der Netzwerkkette innerhalb einer virtuellen Röhre zurückzuführen (vgl. Staudinger 2007). Der elastoplastische Anteil des Rabkin-Modells beruht auf einem Modell von Palmov (1998), welches aus einer Parallelschaltung unendlich vieler Prandtl-Elemente (Reihenschaltung einer Hooke'schen Feder und eines Saint-Venant-Reibelements) besteht. Zur Berücksichtigung von zeitabhängigen Effekten wird ein weiterer viskoelastischer Anteil ergänzt. Dieser setzt sich aus einem generalisierten Maxwell-Modell zusammen. Für eine weiterführende Vertiefung des Rabkin-Modells wird auf die Arbeit von Freund (2013) und die Veröffentlichung von Gelke \& Ihlemann (2016) verwiesen. Dort finden sich neben einer detaillierten Beschreibung aller wichtigen Materialgleichungen, auch Anpassungen zum Speicher- und Verlustmodul.

Nachfolgend wird ein Parametersatz des Rabkin-Modells verwendet, der von M. Sc. Gelke an der Professur Festkörpermechanik mit Hilfe der Optimierungstoolbox von Matlab (Levenberg-Marquardt-Verfahren) indentifiziert wurde. In der Abbildung 5.1 sind sowohl die Messdaten für gefüllten EPDM (schwarzer Kurvenverlauf) als auch die zugehörige Anpassung (roter Kurvenverlauf) dargestellt. Zur besseren Veranschaulichung der großen

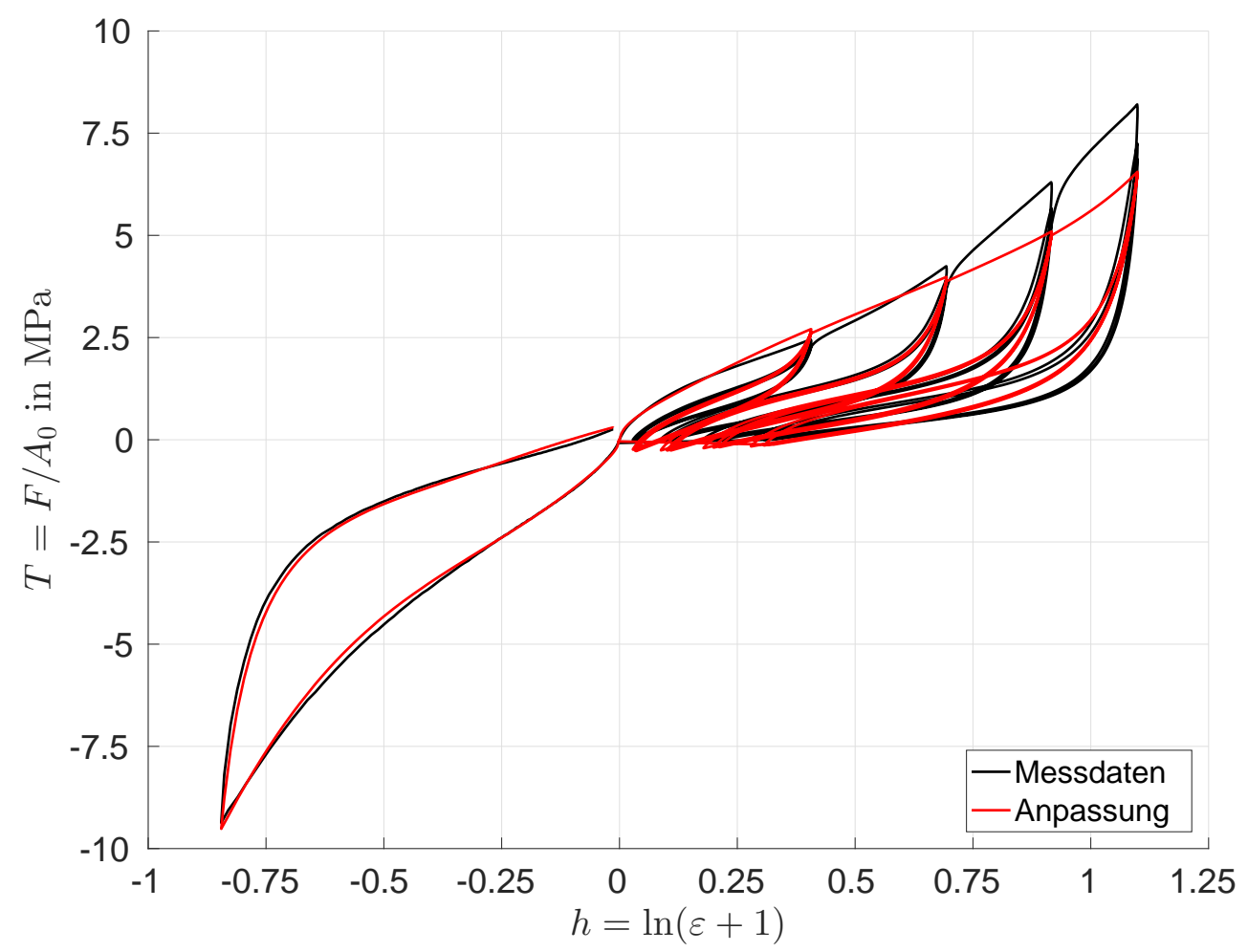

Abb. 5.1 - Parameteranpassung des Rabkin-Modells an die homogenen Zug/Druckversuche (Messungen für gefüllten EPDM, Anpassung von M. Sc. Gelke an der Professur Festkörpermechanik, TU Chemnitz) 
Zug- bzw. Druckstufen wird hier das logarithmische Hencky-Dehnungsmaß $h=\ln (\varepsilon+1)$ aufgetragen. Für eine Anpassung des Rabkin-Modells können dabei verschiedene Komplexitätsstufen ausgewählt werden. Die Anpassung, die hier durchgeführt wurde, erfolgte bei der höchsten Komplexitätsstufe (mit Berücksichtigung der Elastoplastizität, der Viskoelastizität und des Mullins-Effekts). Es sei an dieser Stelle darauf hingewiesen, dass der Druckbereich etwas stärker gewichtet wurde als der Zugbereich. Dies erklärt auch, warum die Anpassung im Druckbereich (vgl. Abbildung 5.1) wesentlich besser ausgefallen ist als im Zugbereich. Hier sind insbesondere bei den größten Zugstufen zunehmende Abweichungen in den Hysteresespitzen zu verzeichnen. Nichtsdestotrotz besitzt die Anpassung einen hohen Gütegrad, bei der alle grundlegenden Eigenschaften von Elastomeren wie Hysterese, Mullins-Effekt und bleibende Dehnung sehr gut abgebildet werden.

Als nächstes wird mit dem angepassten Stoffgesetz eine Probekörpersimulation durchgeführt. Es soll dabei überprüft werden welche Abweichung bei Verwendung der neuen Messstrategie im Vergleich zur homogenen Simulation mit einem finiten Element (entspricht der Anpassung in Abbildung 5.1 entsteht. Bei einem idealen Probekörper dürfte zwischen der Probekörpersimulation und der homogenen Simulation mit einem Element kein Unterschied mehr auftreten. In der Abbildung 5.2 ist der Vergleich zwischen Probekörper- und homogener Simulation mit einem Element dargestellt. Anhand der Abbildung wird deut-

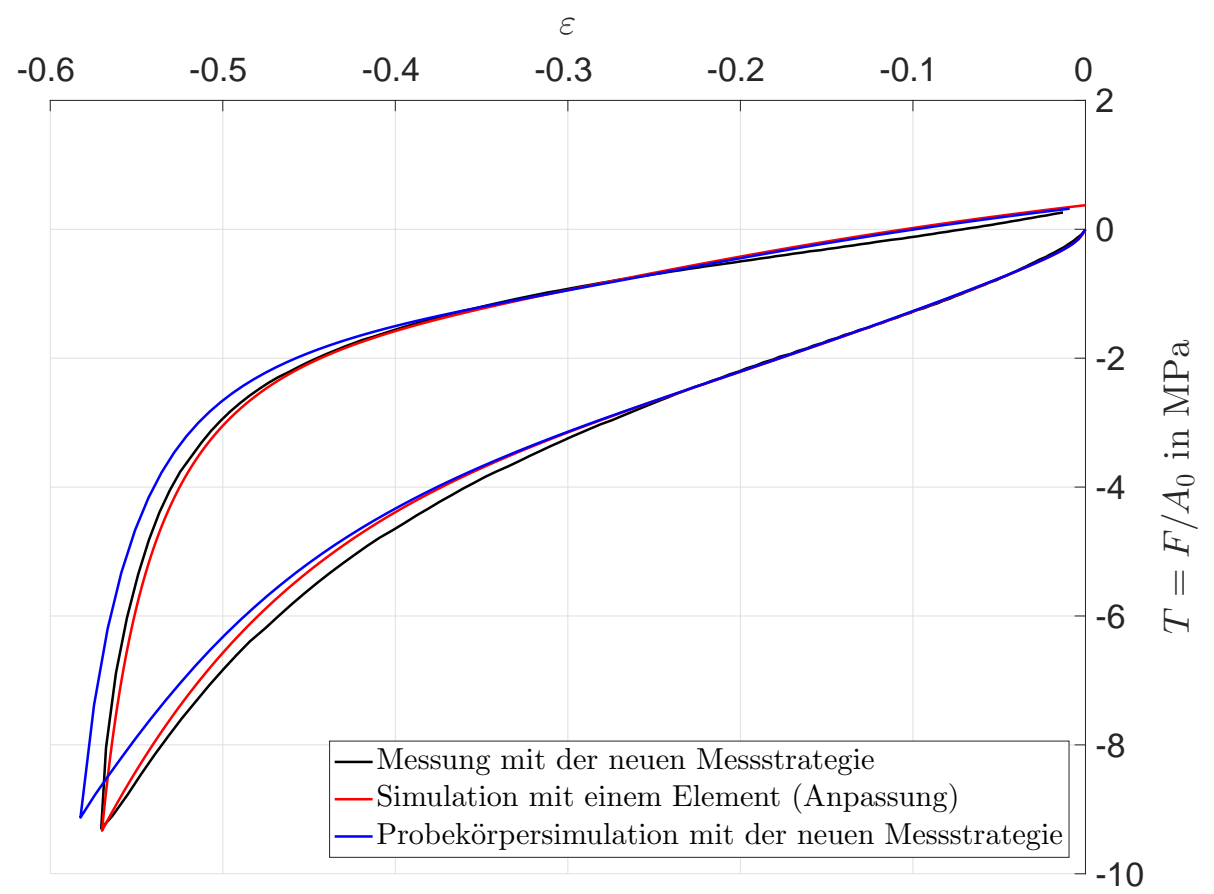

Abb. 5.2 - Vergleich der Probekörper und der homogenen Simulation (Messung für gefüllten EPDM, vgl. Abbildung 5.1

lich, dass das Probekörper-Setup im Druckbereich eine zutreffende Prognose liefert. Die Abweichungen, die hier noch auftreten, sollen im Folgenden näher diskutiert werden. Zunächst kann festgehalten werden, dass erst bei einer Stauchung größer $40 \%$ Unterschiede 
zur homogenen Lösung (Simulation mit einem Element) sichtbar sind. Mit zunehmender Stauchung wird die Annahme der homogenen Druckdeformation immer stärker verletzt, was die leicht anwachsenden Abweichungen erklärt. Abgesehen davon liefert das entwickelte Probekörper-Setup aber hervorragende Messkurven für diesen großen Druckbereich. Mit dem angepassten Stoffgesetz können weiterhin in direkter Weise inelastische Effekte mit dem Probekörper-Setup simuliert werden. Zum Beispiel kann der Einfluss der Restverformung untersucht werden. Durch das veränderte Längen-Durchmesserverhältnis wirken sich Imperfektionen unterschiedlich stark auf das Stabilitätsverhalten des Probekörpers aus.

Im Weiteren soll anhand einer Simulation überprüft werden, wie gut sich das globale Kraft-Wegverhalten (Traversenweg) im Vergleich zur realen Messung vorhersagen lässt. In der Abbildung 5.3 ist der globale Vergleich dargestellt. Aus der Abbildung wird deutlich,

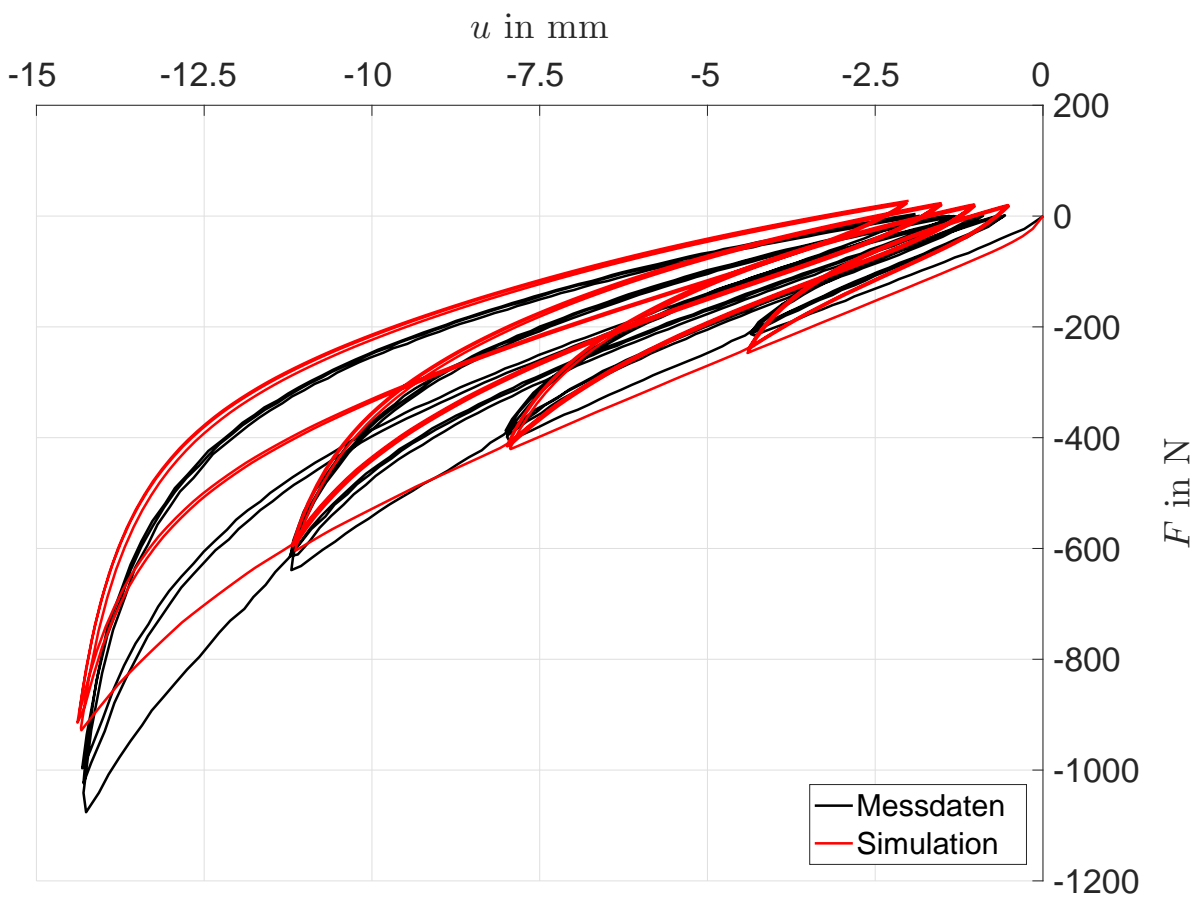

Abb. 5.3 - Vergleich des globalen Kraft-Wegverhaltens (Traversenweg) zwischen Messung für gefüllten EPDM und Simulation

dass das globale Verhalten nur näherungsweise wiedergegeben werden kann. Die Abweichungen, die aus dem Kraftverhalten resultieren, sollen im Folgenden näher diskutiert werden. Zunächst einmal muss darauf hingewiesen werden, dass die Probekörper, die für die Identifikation von Materialparametern und für die Vergleichsmessung verwendet wurden, aus nachfolgenden Materialchargen stammen. Erfahrungsgemäß können hier leichte Abweichungen durch den Herstellungsprozess auftreten. Diese Vermutung wird auch dadurch gestützt, dass schon bei kleinen Verschiebungen die Grundsteifigkeit leicht abweicht. Weiterhin muss berücksichtigt werden, dass die Probekörpersimulation bewusst mit einem vereinfachten axialsymmetrischen Modell ohne Hantelkopf durchgeführt wurde. Mit dieser 
Abstraktionsstufe und der Tatsache, dass keine weitere Anpassung an diesen Messdaten vorgenommen wurde, lässt sich das globale Kraft-Wegverhalten in erster Näherung gut abschätzen. Als besonderes Augenmerk sei hier die Vorhersage der bleibenden Dehnung im Nulldurchgang zu nennen, die erstaunlich gut wiedergegeben wird (vgl. dazu Abbildung 5.1. wo diesbezüglich keine Anpassung stattfand).

Abschließend wird mit dem angepassten Stoffgesetz (Anpassung erfolgte an die Zug/Druckkurven) ein symmetrischer Scherversuch durchgeführt, indem die gleichen Schermaßstufen wie im Experiment angefahren werden (vgl. Abbildung 4.19). Ziel der Untersuchung ist es zu überprüfen, wie gut sich mit einer homogenen Anpassung ein Scherversuch vorhersagen lässt. In der Abbildung 5.4 ist der Vergleich zwischen dem Experiment und der Simulation des Scherversuches für gefüllten EPDM (gefüllter EPDM210 72 ShA) dargestellt. Anhand der Abbildung wird deutlich, dass der Scherversuch in erster Näherung sehr

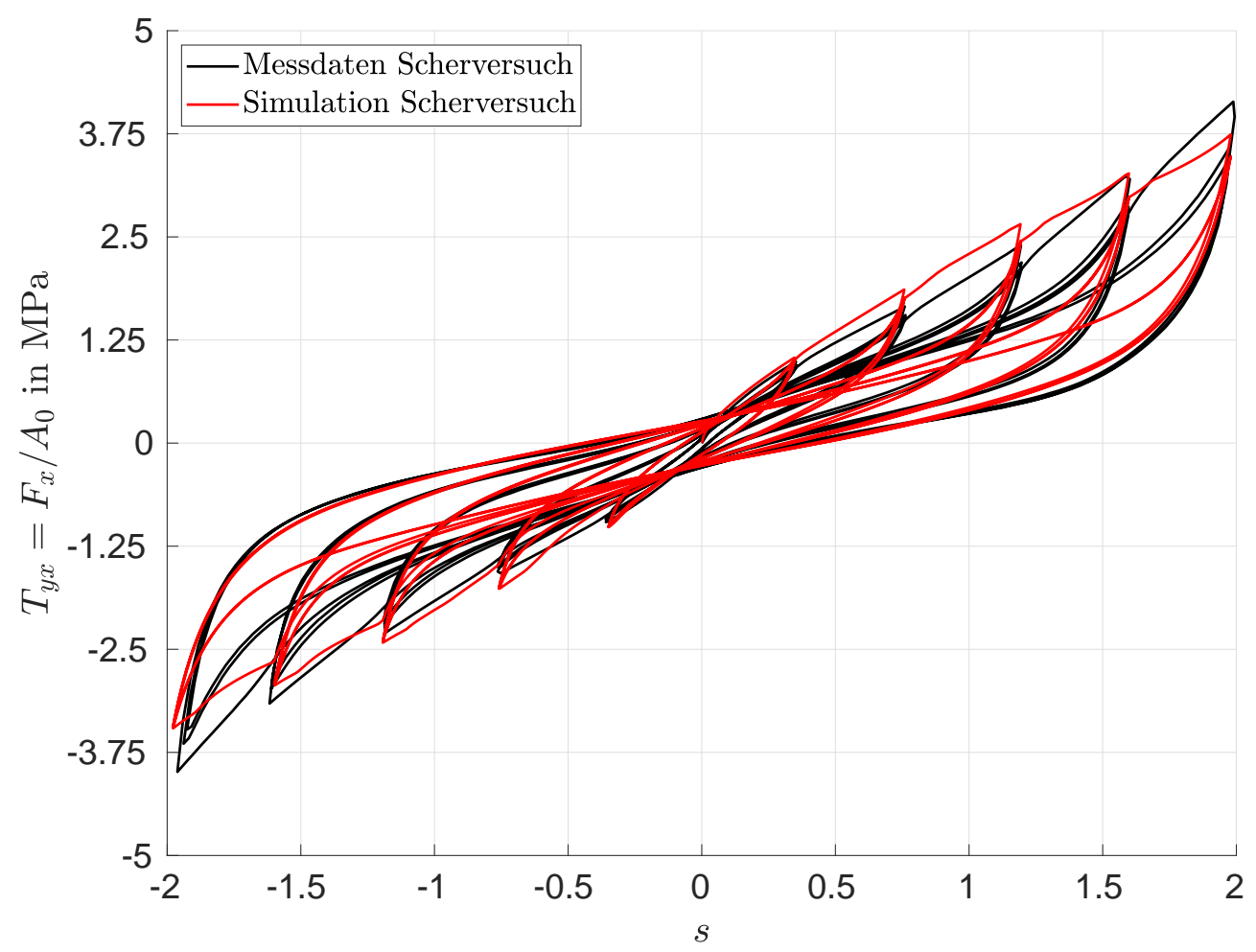

Abb. 5.4 - Vergleich zwischen Scherversuch für gefüllten EPDM (vgl. Abbildung 4.19) und homogener Simulation (Anpassung erfolgte an Zug/Druckkurven)

gut vorhergesagt werden kann. Bei den Belastungskurven ist zu erkennen, dass die Messung einen leicht progressiven Verlauf und die Simulation einen leicht degressiven Verlauf aufweist. Demgegenüber zeigen die Entlastungskurven eine sehr gute Übereinstimmung. Es sei hier aber nochmal explizit darauf hingewiesen, dass für das angepasste Stoffgesetz kein Scherversuch berücksichtigt worden ist. Für eine spätere Bauteilsimulation, beispielsweise Fahrwerksbuchsen, sollte jedoch darauf geachtet werden, dass der Anpassung verschiedene homogene Datensätze mit verschiedenen Belastungsarten zugrunde liegen (vgl. Schellen- 
berg 2017).

\section{Zusammenfassung der Anpassung eines Stoffgesetzes:}

Mit Hilfe homogener Standardversuche konnte zunächst ein viskoelastoplastisches Stoffgesetz angepasst werden. Diese Anpassung ermöglicht den qualitativen Vergleich zwischen der homogenen Simulation mit einem Element und der Simulation mit dem ProbekörperSetup. Dabei konnte festgehalten werden, dass das Probekörper-Setup auch bei großen Stauchungen zu nahezu homogenen Messergebnissen führt. Die Anpassung ermöglicht darüber hinaus auch die Vorhersage von recht komplexen Bauteilverhalten. Beispielsweise können inelastische Effekte wie Restverformung in einem nächsten Iterationsschritt der Halterungsentwicklung mit berücksichtigt werden.

\subsection{Untersuchung des ratenabhängigen Materialverhaltens von Elastomeren}

Insbesondere bei zyklischen Belastungen zeigen technische Elastomere ein niedriges ratenabhängiges Materialverhalten über einen großen Frequenzbereich. Dieser Effekt lässt sich sehr gut im direkten Vergleich zwischen einem vorgegebenen Zickzackverlauf und einem Sinusverlauf veranschaulichen (vgl. Besdo u. a. 2003). Hier zeigt sich an den Umkehrpunkten eine unterschiedlich stark ausgeprägte Hysteresespitze. Bei der Modellierung von Stoffgesetzen für technische Elastomere wird aber oftmals von einem ratenunabhängigen Materialverhalten ausgegangen (vgl. MorPH-Modell (Ihlemann 2003) und RabkinModell (Freund 2013)). Dies führt zwangsweise dazu, dass wichtige geschwindigkeitsabhängige Effekte nicht abgebildet werden können. Ziel dieses Abschnittes ist es, mit Hilfe der erweiterten Prüfmaschinensteuerung (siehe Abschnitt 3.4.2) und dem neu entwickelten Probekörper-Setup die geschwindigkeitsabhängigen Effekte von technischen Elastomeren noch eingehender zu untersuchen. Auf der Grundlage dieser Messergebnisse können dann auch zeit-invariante Materialmodelle getestet und miteinander verglichen werden (vgl. Donner u.a. 2017). Diese Untersuchungen bilden somit eine wichtige experimentelle Datenbasis für die Entwicklung neuer Stoffgesetze. Zunächst werden mit Hilfe der erweiterten Prüfmaschinensteuerung Prüfabläufe modelliert, die zu einem ratenabhängigen Materialverhalten führen. Dazu wird ein harmonischer Sinusverlauf mittels ungerader Potenzen wie folgt modifiziert:

$$
u(t)=\hat{u} \sin ^{n}(\omega t) \text { mit } n=1,3,5,7 .
$$

In der Abbildung 5.5 sind die Sinusverläufe mit den unterschiedlichen Potenzen dargestellt. Anhand der Abbildung wird deutlich, dass die Modifizierung dazu führt, dass es Bereiche im Sinusverlauf gibt, in denen ein sehr flacher Anstieg entsteht und Bereiche, wo dieser sehr stark ausgeprägt ist. Diese Prüfverläufe $u(t)$, die im Weiteren mit der Traverse verfahren werden, sind in der Abbildung 5.5 bereits in der entsprechenden Wegskalierung 


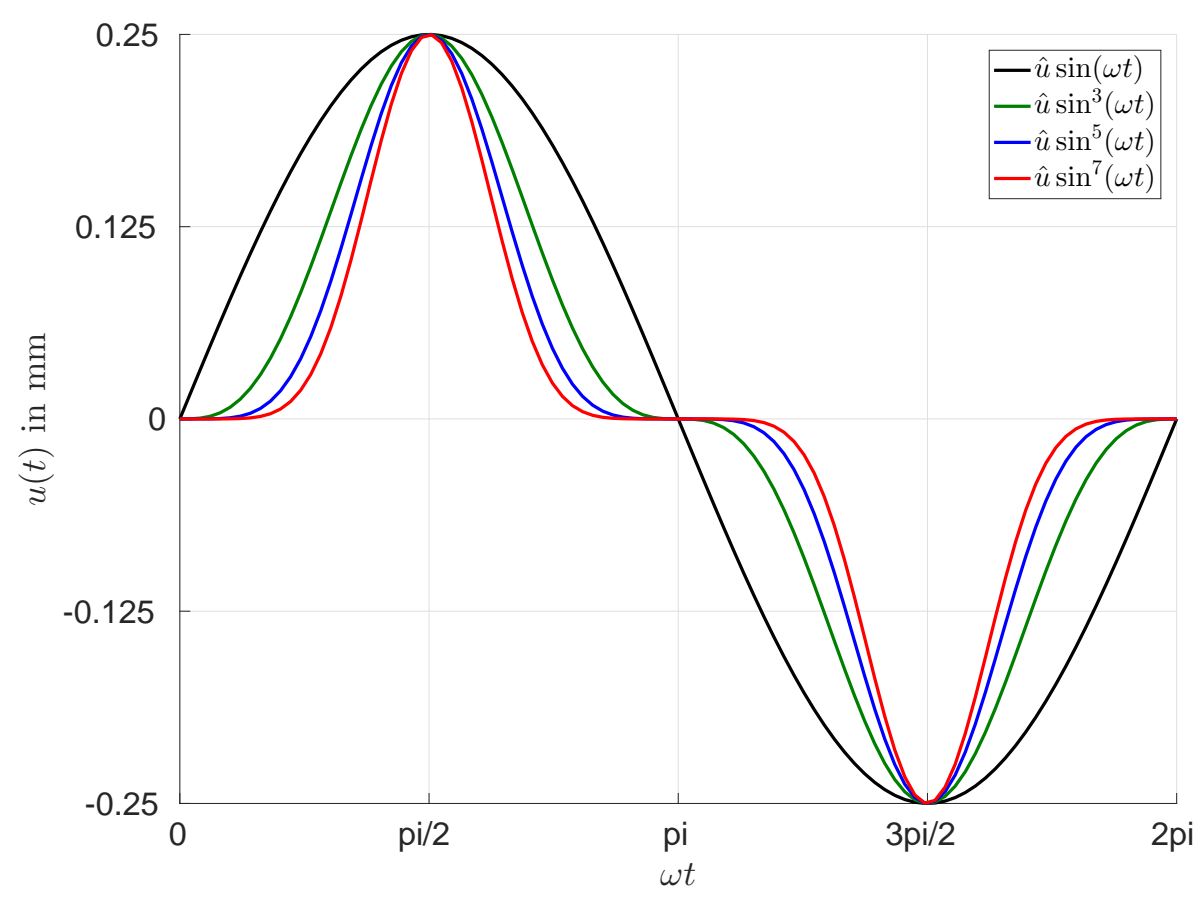

Abb. 5.5 - Darstellung der Sinusverläufe mit unterschiedlichen Potenzen zur Untersuchung des ratenabhängigen Materialverhaltens (Simulationsergebnis$\mathrm{se})$

dargestellt. Aufgrund der kleinen Verfahrwege wird für das Probekörper-Setup der hochpräzise MultiXtens als Messsystem verwendet (vgl. Abbildung 5.6. Da dieses System für

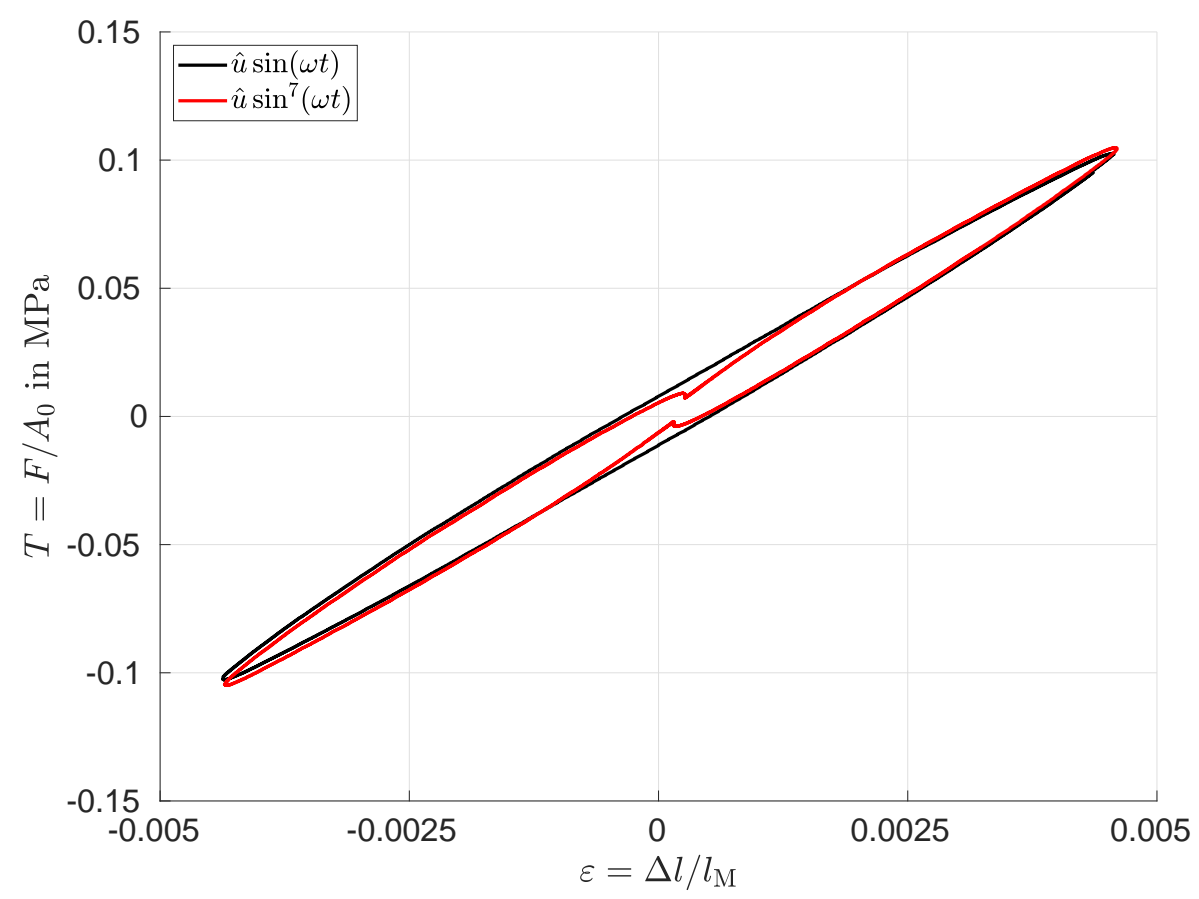

Abb. 5.6 - Spannungs-Dehnungsantwort der Sinusverläufe mit unterschiedlichen Potenzen für den Zug-/Druckprobekörper (Messungen für gefüllten EPDM) 
den Scherprobekörper nicht angewendet werden kann, kommt hier das 3D DIC-Messsystem zum Einsatz. Für ein geringeres Verhältnis von Rauschen zu Messsignal wurden diesbezüglich etwas größere Amplituden verwendet. Zur besseren Übersicht werden in Abbildung 5.6 die experimentellen Spannungs-Dehnungsantworten resultierend aus dem harmonischen Signal mit dem (resultierenden) Sinusverlauf der Potenz 7 verglichen. Es sei an dieser Stelle darauf hingewiesen, dass im Spannungs-Dehnungsdiagramm nur die stationären Zyklen dargestellt sind. Aus der Abbildung 5.6 lassen sich eine Reihe interessanter Erkenntnisse gewinnen. Zunächst wird deutlich, dass sich im Ursprung eine eingeschnürte Hystereseform ausbildet, die mit zunehmender Potenz des Eingangssignals immer stärker in den Ursprung wandert. Dieser Effekt kann dadurch erklärt werden, dass durch die sehr flachen Übergangsphasen in den Sinusverläufen (Haltephasen) ein Relaxieren des Materials stattfindet. Weiterhin ist zu beobachten, dass keine perfekte Punktsymmetrie innerhalb des Ursprunges vorliegt. Diese leichte Asymmetrie ist auf das unterschiedliche Zug-Druckverhalten von technischen Elastomeren zurückzuführen. Um diese Behauptung zu stützen, sind die gleichen Sinusverläufe für den Scherprobekörper gefahren worden (siehe Abbildung 5.7). Betrachtet man die Umkehrpunkte der Hysteresen, so ist für die modifizierten Signale

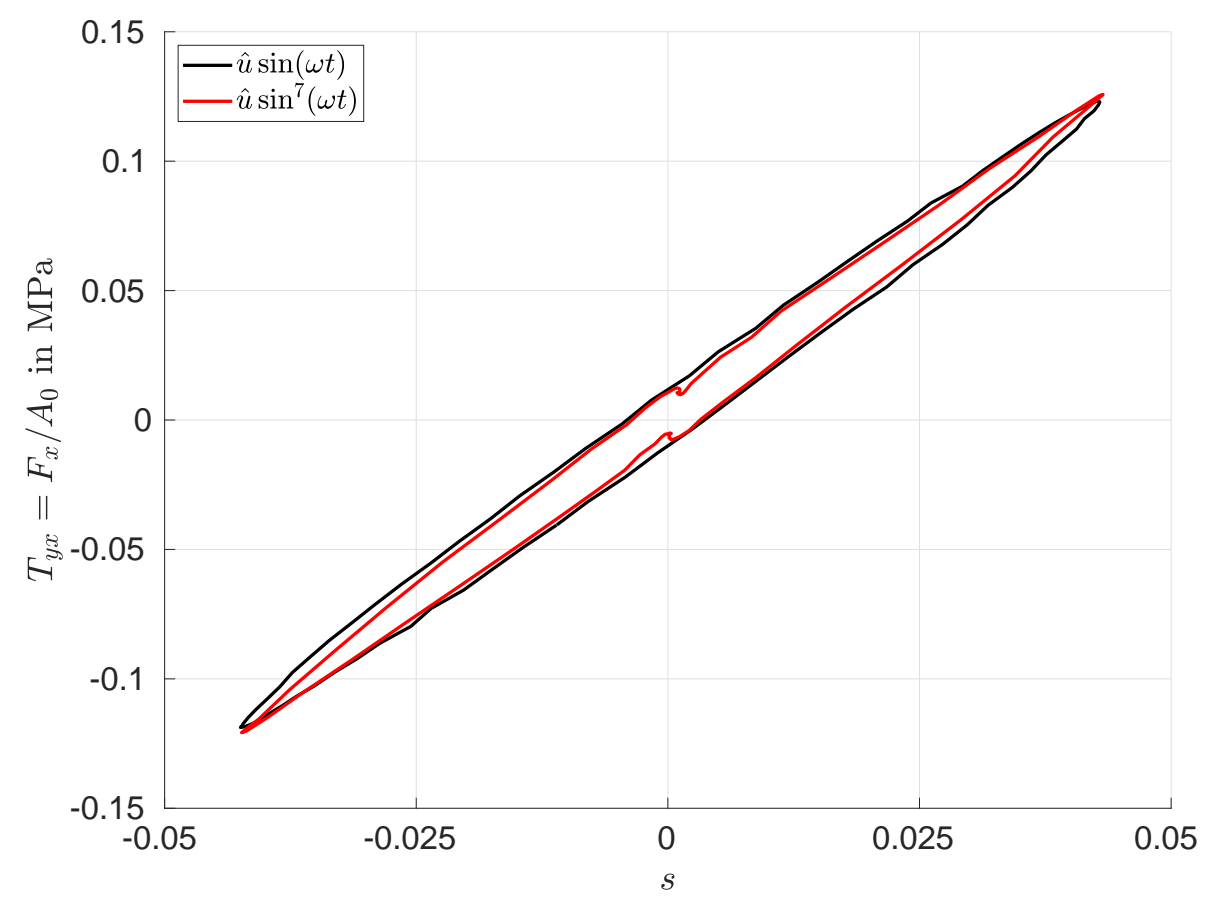

Abb. 5.7 - Spannungs-Schermaßantwort der Sinusverläufe mit unterschiedlichen Potenzen für den Scherprobekörper (Messungen für gefüllten EPDM)

ein steilerer Anstieg der Spitzen zu verzeichnen. Dieser Effekt kann durch den steileren Anstieg der Amplituden im modifizierten Sinusverlauf erklärt werden (vgl. Abbildung 5.5).

Im Folgenden soll das harmonische Sinussignal in der Weise modifiziert werden, dass sich eine größtmögliche Ratenabhängigkeit gegenüber dem herkömmlichen Sinusverlauf einstellt (vgl. Donner u. a. 2017). Dabei ergeben sich zwei prinzipielle Modellierungsvarianten. Bei 
der ersten Variante soll ein stark variierender Übergang zwischen den beiden Amplitudenspitzen erfolgen, im Weiteren als Sägezahnverlauf bezeichnet. In der zweiten Variante soll ein größtmöglicher Unterschied bei den Amplitudenformen erzielt werden, nachfolgend als Kettenradverlauf bezeichnet. Die mathematische Beschreibung dieser verzerrten Verläufe erfolgt in der Gleichung (5-3). Dabei ist zu beachten, dass für den Sägezahnverlauf eine Sinusfunktion verwendet wird und für den Kettenradverlauf eine Cosinusfunktion:

$$
u(t)=\hat{u} \sin (z(\omega t)) \begin{cases}\text { mit: } z(\omega t)=\frac{\omega t}{v^{2}(1-2 \omega t)+2 \omega t} & \text { für: } 0 \leq \omega t \leq \pi \\ \text { mit: } z(\omega t)=\frac{\omega t-1 / 2}{2 / v^{2}(1-\omega t)+2 \omega t-1}+\frac{1}{2} & \text { für: } \pi<\omega t<2 \pi\end{cases}
$$

Der Parameter $v$ beschreibt dabei den Grad der Verzerrung der herkömmlichen Sinus- bzw. Cosinusfunktion. Beispielsweise führt eine Verzerrung mit Wert $v=1 \mathrm{zu}$ dem harmonischen Ausgangssignal. In der Abbildung 5.8 und der Abbildung 5.10 sind die Sägezahnund Kettenradverläufe für die Verzerrungen $v=1$ bis 4 dargestellt. Zur besseren Ver-

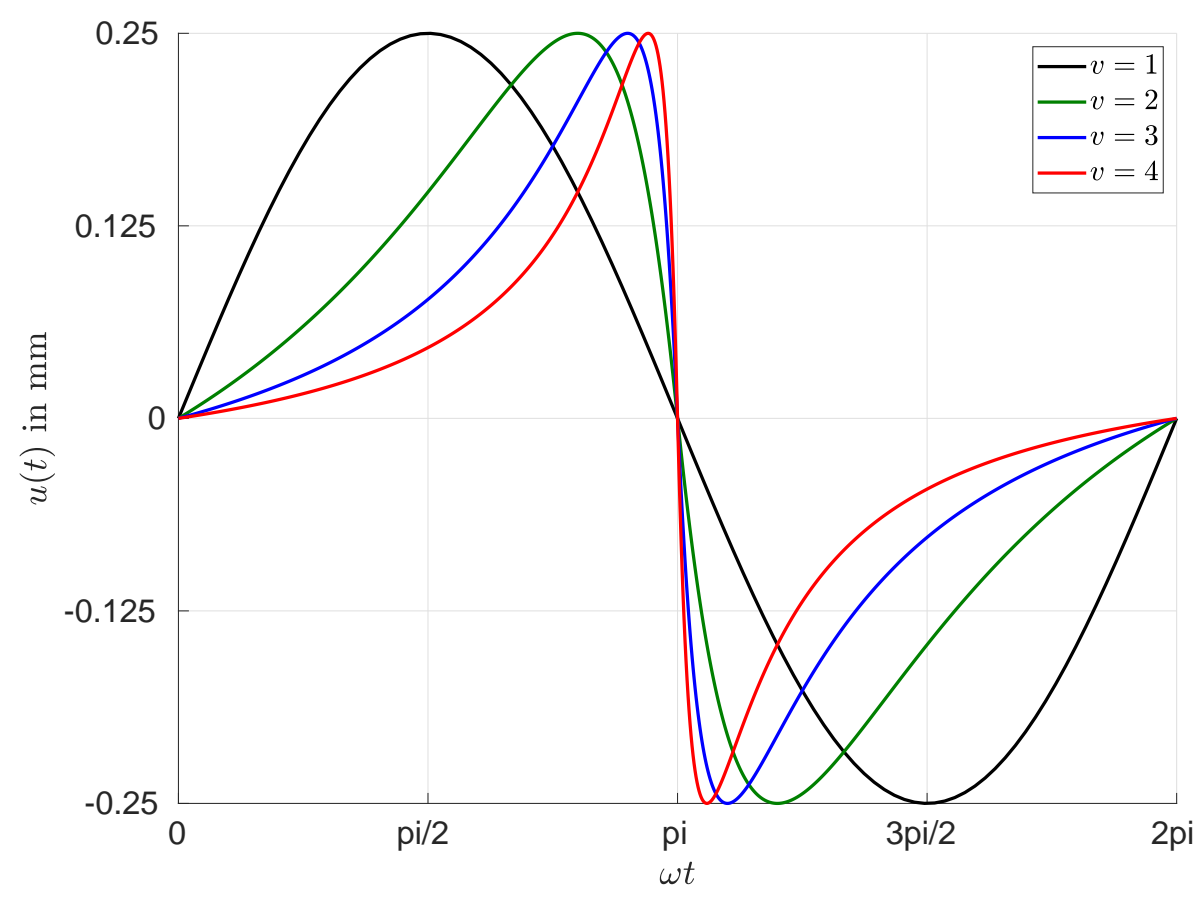

Abb. 5.8 - Darstellung der verzerrten Sinusverläufe (Sägezahnverlauf) zur Untersuchung des ratenabhängigen Materialverhaltens (Simulationsergebnisse)

gleichbarkeit werden den verzerrten Eingangssignalen gleich die zugehörigen SpannungsDehnungsantworten beigefügt (vgl. Abbildung 5.9 und Abbildung 5.11). Zunächst soll dazu auf die Abbildung 5.9 näher eingegangen werden. Hier wird deutlich, dass der Sägezahnverlauf zu einer unsymmetrischen Hystereseform führt. Diese weißt im Zugbereich einen schmalen und zum Druckbereich einen größer werdenden Hysteresebauch auf. Weiterhin ist zu beobachten, dass sich der untere breitere Hysteresebauch auch unterhalb der 


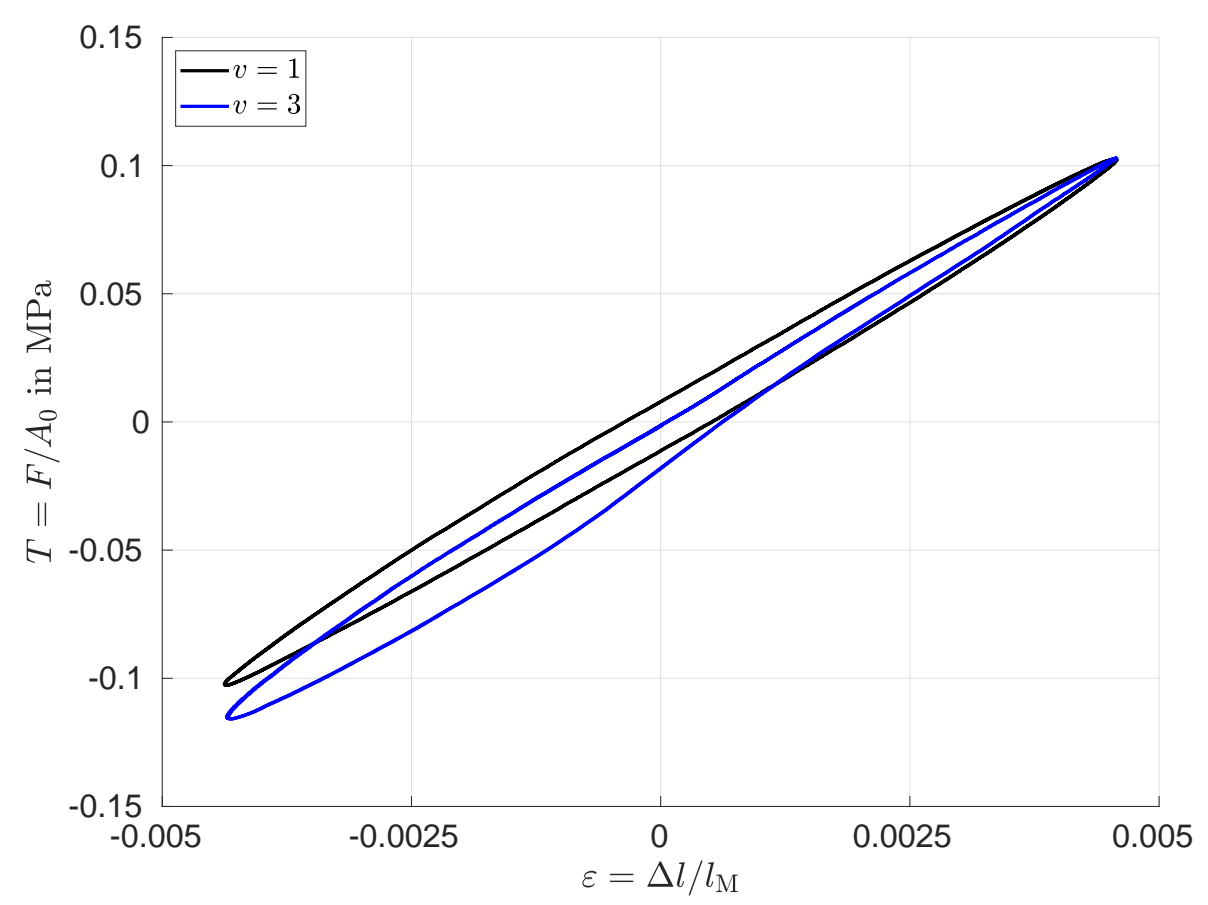

Abb. 5.9 - Spannungs-Dehnungsantwort der verzerrten Sinusverläufe (Sägezahnverlauf) für den Zug-/Druckprobekörper (Messungen für gefüllten EPDM)

gewöhnlichen Hysterese, resultierend aus dem harmonischen Sinussignal, befindet. Diese starke Asymmetrie ist nicht zuletzt auf die stark variierenden Übergangsbereiche zwischen den Amplitudenspitzen zurückzuführen. Es kann dabei ein sehr schneller und ein sehr langsamer Pfad detektiert werden. Diese großen Geschwindigkeitsunterschiede führen zwangsweise dazu, dass das Material unterschiedlich stark relaxiert. Zur besseren Übersicht wurde hier nur ein verzerrtes Signal $(v=3)$ dem harmonischen gegenübergestellt. Es kann jedoch festgehalten werden, dass mit zunehmender Verzerrung $v$ der obere Hysteresebauch schmaler und der untere breiter wird. Interessanterweise scheint auch die dissipierte Energie vom Grad der Verzerrung abzuhängen, weil der Inhalt der Hystereseflächen unterschiedlich groß ausgeprägt ist. Im Anhang ist der gleiche Versuchsablauf für den Scherprobekörper dargestellt (siehe Abbildung A.5. Alle eben beschriebenen Effekte lassen sich auch hier wiederfinden. Allerdings sind diese in der einfachen Scherung nicht so stark ausgeprägt.

Als nächstes sollen die Messergebnisse der Abbildung 5.11 näher diskutiert werden. Zunächst wird ersichtlich, dass sich die stationären Hysteresen mit zunehmender Verzerrung $v$ parallel zur Referenzhysterese nach unten bewegen. Die Hysteresefläche scheint dabei konstant zu bleiben. Bei genauerer Betrachtung der Abbildung 5.11 fällt auf, dass die oberen Umkehrpunkte enger zusammen liegen als die unteren. Dieser Effekt soll anhand der Abbildung 5.10 näher untersucht werden. Hier wird deutlich, dass der obere Amplitudenverlauf aufgrund seiner ausgedehnten Struktur zu einem starken Relaxieren des Materials im oberen Umkehrbereich führt. Der danach sehr schnell wechselnde Verlauf in den unte- 


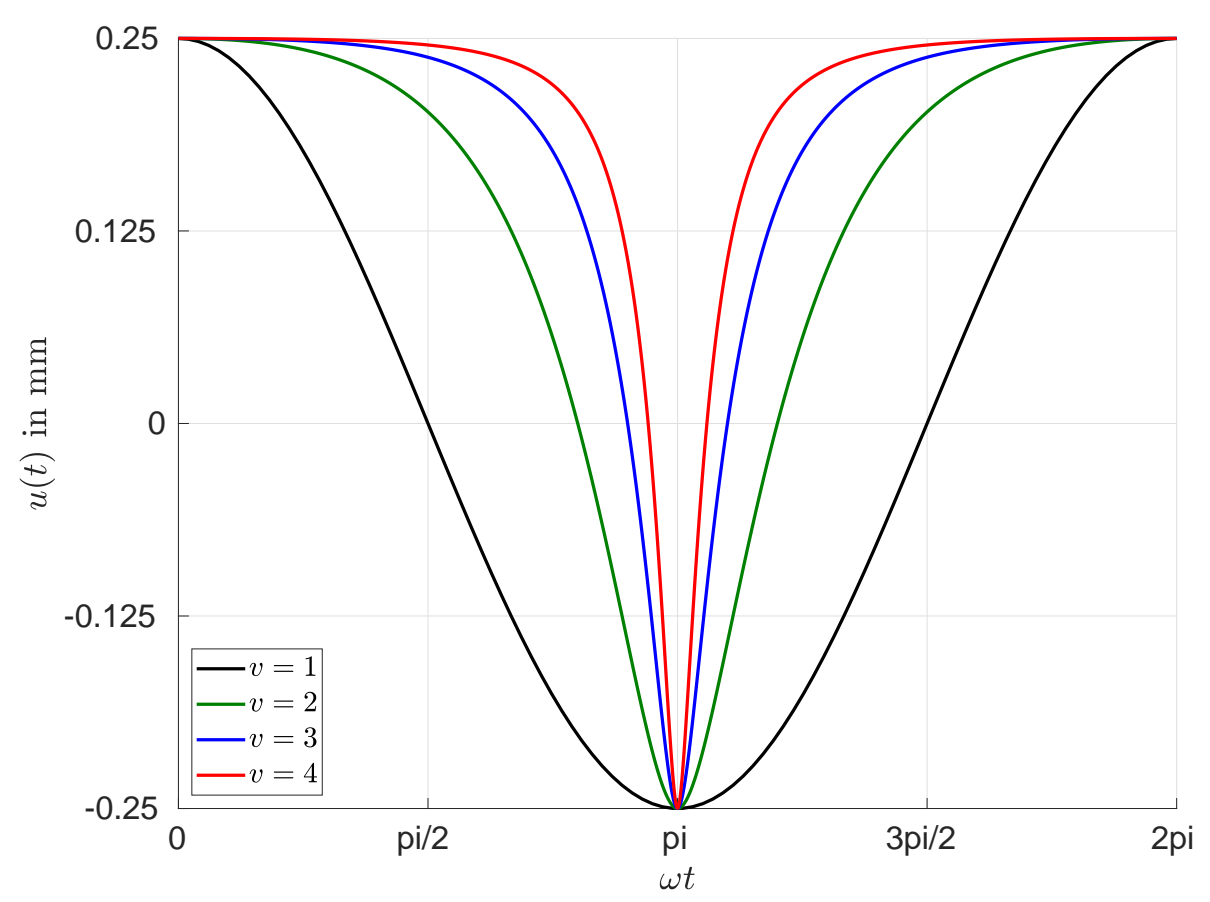

Abb. 5.10 - Darstellung der gestreckten Cosinusverläufe (Kettenradverlauf) zur Untersuchung des ratenabhängigen Materialverhaltens (Simulationsergebnisse)

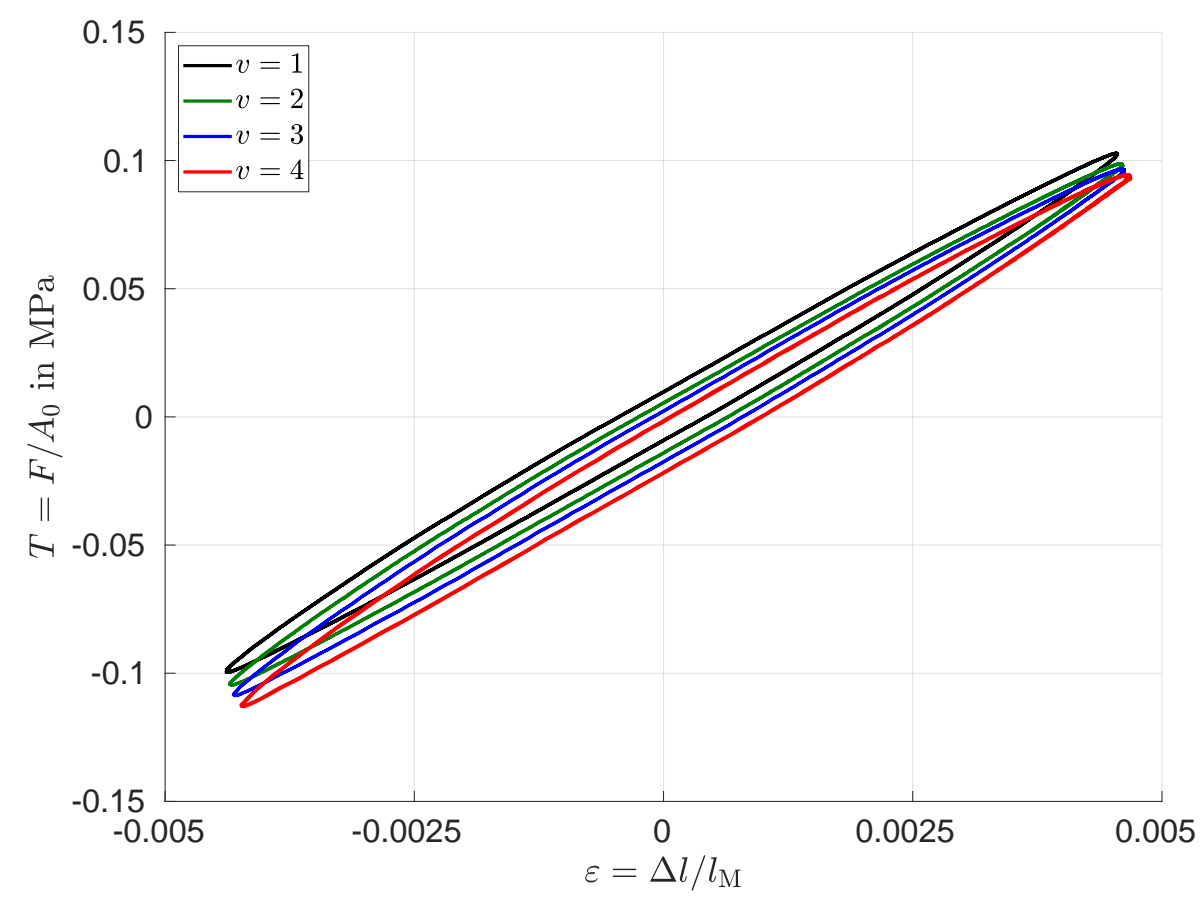

Abb. 5.11 - Spannungs-Dehnungsantwort der gestreckten Cosinusverläufe (Kettenradverlauf) für den Zug-/Druckprobekörper (Messungen für gefüllten EPDM)

ren Umkehrpunkt verhindert zwangsweise ein langsames Zurückrelaxieren des Materials zur Referenzhysterese. Die Versuche mit den Sägezahn- bzw. Kettenradverläufen machen 
deutlich, wie stark ratenabhängig sich technische Elastomere verhalten.

\section{Zusammenfassung der Untersuchung zur Ratenabhängigkeit:}

Anhand der messtechnischen Befunde kann festgehalten werden, dass technische Elastomere (hier: gefüllter EPDM) ein stark ratenabhängiges Materialverhalten zeigen. Obwohl die gewählte Amplitude und die verwendete Prüffrequenz in allen Versuchen konstant gehalten wurde, führen die unterschiedlichen Dehnraten zu einer starken Beeinflussung der Hystereseform. Mit Hilfe dieser Messergebnisse können unter anderem auch zeit-invariante Materialmodelle, wie sie in der Veröffentlichung von Donner u.a. (2017) vorgestellt wurden, getestet und validiert werden. Die hier zugrunde gelegte Datenbasis bietet somit eine ideale Vergleichsmöglichkeit für neu entwickelte Stoffgesetze. Es sei an dieser Stelle nochmal explizit darauf hingewiesen, dass es mit dem neu konzipierten Probekörper-Setup ebenso denkbar wäre, die Ratenabhängigkeit bei unterschiedlichen Zug- bzw. Druckstufen zu untersuchen. Im Rahmen der messtechnischen Realisierung wird empfohlen, die gewünschten Zug- bzw. Druckstufen mit der Standardsteuerung anzufahren und dann auf die erweitere Prüfmaschinensteuerung zu wechseln. Somit ist gewährleistet, dass die verzerrten Eingangssignale bestmöglich in dem Bereich von $\pm 10 \mathrm{~V}$ aufgelöst sind.

\subsection{Implementierung des Probekörper-Setups in den Industriealltag}

Nachfolgend soll das entwickelte Probekörper-Setup, wie es im Rahmen dieser Arbeit vorgestellt wurde, für den Industriealltag zugänglich gemacht werden. Die Zielstellung dabei ist, dass das Probekörper-Setup zum einen für die tägliche Qualitätskontrolle der verschiedenen Materialmischungen, zum anderen für die Parameteranpassung von Stoffgesetzen eingesetzt werden kann. Die wichtigsten Neuerungen dabei sind:

- Die Qualitätskontrolle der verschiedenen Materialmischungen kann auf Grundlage phänomenologischer Eigenschaften technischer Elastomere durchgeführt werden.

- Bisherige verschiedene Zug- bzw. Druckprobekörper können durch das neue Probekörper-Setup ersetzt werden (Reduzierung der Probekörpertypen).

- Der zur Verfügung stehende Messbereich wurde für Zug-/Druckmessungen deutlich erweitert.

- Die Homogenität im Messbereich, vorzugsweise im Druck, wurde deutlich verbessert.

- Es können in direkter Weise homogene Messdaten für die Stoffgesetzanpassungen herangezogenen werden.

Um das Probekörper-Setup in den Industriealltag implementieren zu können, muss im Wesentlichen der gesamte Ein- und Ausbauprozess beschleunigt werden (Minimierung der 
Taktzeit für eine standardisierte Prüfung). Dies wird im Folgenden durch eine automatische Einspannvorrichtung realisiert. In der Abbildung 5.12 ist die automatische Einspannvorrichtung, wie sie bei der Firma Vibracoustic GmbH eingesetzt wird, dargestellt (vgl. Tölle u. a. 2018). Die Grundidee zum Einspannen des Probekörpers geht dabei auf
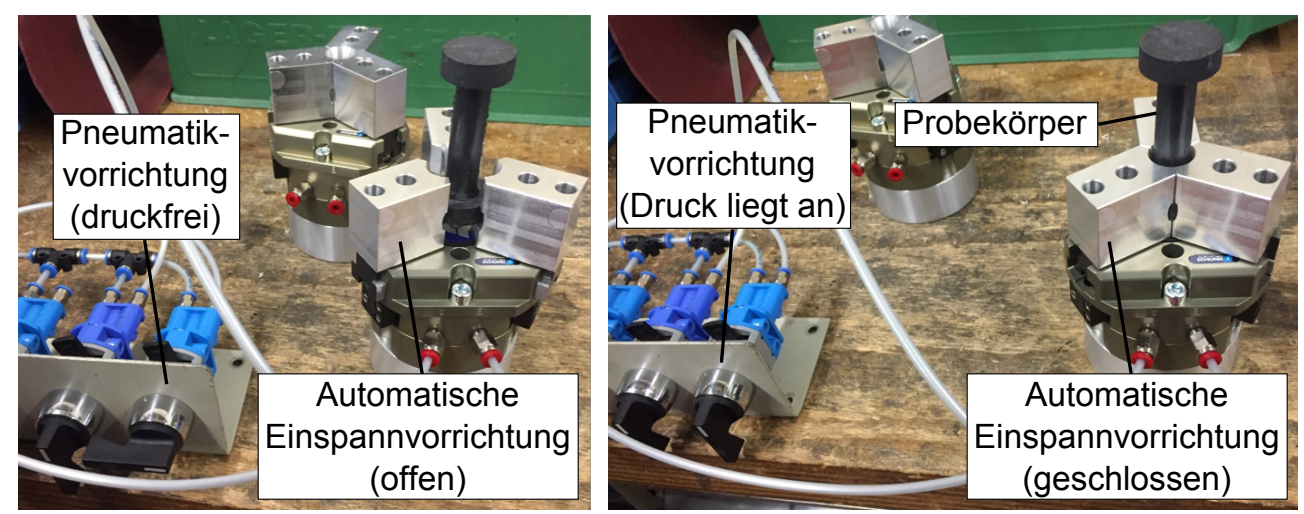

Abb. 5.12 - Automatische Einspannvorrichtung zur Implementierung des Probekörper-Setups in den Industriealltag (Abbildung von Dr. Folke J. Tölle aus der Firma Vibracoustic GmbH)

ein Dreibackenfutter von Drehmaschinen zurück. Die Zustellung der Halterungssegmente wird dabei über eine Pneumatikvorrichtung realisiert. Neben der erheblichen Zeitersparnis ist durch das Dreibackensystem sichergestellt, dass der Probekörper zentrisch eingebaut wird. Es sollte allerdings zuvor darauf geachtet werden, dass die automatischen Einspannvorrichtungen koaxial in der Prüfmaschine verbaut sind. Gegebenenfalls kann durch eine Ausrichteinheit eine Korrektur vorgenommen werden (vgl. Abbildung 3.46). Um mit der automatischen Einspannvorrichtung möglichst universell verschiedene Materialmischungen testen zu können, muss der Schrumpf der Probe bestmöglich kompensiert werden. Anhand von Untersuchungen mit gefülltem Naturkautschuk, gefülltem EPDM und FKMMaterial konnte gezeigt werden, dass bei einem Solldurchmesser der Vulkanisationsform von $15 \mathrm{~mm}$ ein Schrumpf in der Größenordnung von $2 \%$ auftritt. Dieser Schrumpf wurde bisher mit entsprechenden Halterungsringen kompensiert. Für zusätzliche Reserven gegenüber unbekannten Materialmischungen, wird eine Kompensation des Schrumpfes von $3 \%$ empfohlen. Vergleichsmessungen mit $2 \%$ und $3 \%$ Kompensation des Schrumpfes haben dabei eine gute Übereinstimmung der Spannungs-Dehnungskurven ergeben.

Abschließend wird für das entwickelte Probekörper-Setup eine automatisierbare Messprozedur vorgestellt, welche für große Zug-/Druckversuche geeignet ist. Das große Ziel ist dabei zum einen die einfache Handhabbarkeit des Messsystems (Bedienung und Auswertung), zum anderen die Gewährleistung einer schnellen und sicheren Messung im Industriealltag (Taktzeit, Qualitätskontrollen). Im Vergleich zu dem bisher verwendeten 3D DIC-Messsystem, mit dem eine Vielzahl wichtiger Größen gemessen werden können, besitzt es die Nachteile, dass es verhältnismäßig aufwendig zu bedienen und sehr kostenintensiv ist. Hinzu kommt, dass die Probenpräparation, die Justage bzw. Kalibrierung des Messsys- 
tems und schlussendlich die Auswertung mit einem gewissen Erfahrungsschatz verbunden sind. Demgegenüber ist die Bedienung eines Längenänderungsaufnehmers, wie dem MultiXtens oder Clip Gage, mit wesentlich weniger Aufwand verbunden. Allerdings eignen sich diese berührenden Systeme in keiner Weise für große Stauchungen. Aus diesem Grund soll ein Messsystem ausgewählt werden, was zum einen auf einem berührungslosen Funktionsprinzip beruht und zum anderen auch eine einfache Handhabbarkeit ermöglicht. Ein sich hierfür eignendes System stellt das High-Speed 2D optische Mikrometer von der Firma KEYENCE dar, welches als Durchlichtverfahren angewendet wird. Befindet sich zwischen der Lichtquelle und einer Abtastplatte ein Probekörper, so kann dessen Außenkontur sehr präzise vermessen werden. Da der Probekörper im Weiteren rotationssymmetrisch ist und für die neue Messstrategie (vgl. Abschnitt 3.3.7) lediglich die Durchmesseränderung benötigt wird, eignet sich das Messsystem in idealer Weise für standardisierte Messungen im Zug-/Druckbereich. In Abbildung 5.13 ist der Versuchsaufbau des Probekörper-Setups mit dem integrierten KEYENCE-Messsystem für einen ausgewählten Zug- und Druckversuch dargestellt. An dieser Stelle sei darauf hingewiesen, dass im Zug der minimale und im

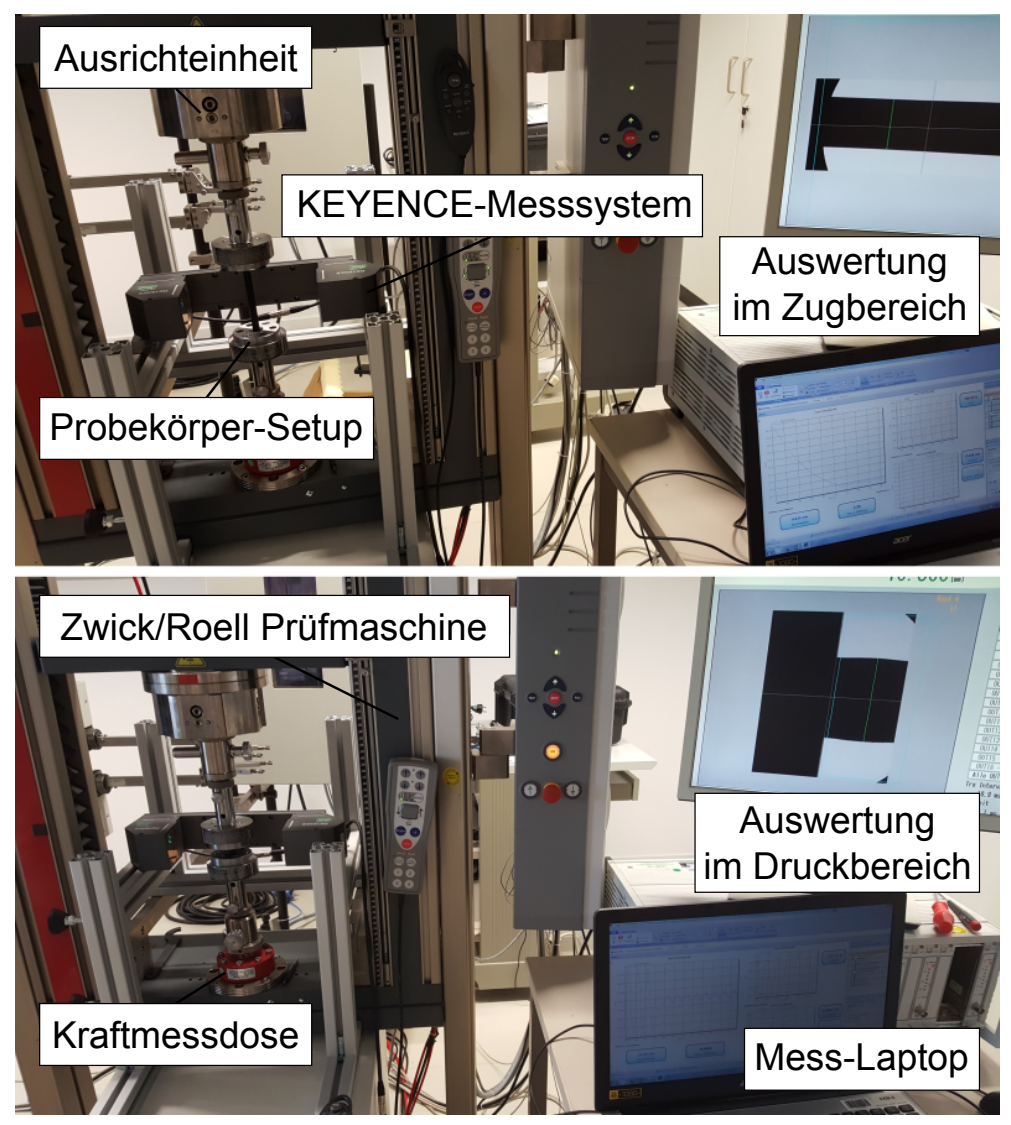

Abb. 5.13 - Versuchsaufbau des Probekörper-Setups an einer elektromechanischen Prüfmaschine mit integriertem KEYENCE-Messsystem: (oben) Zugversuch, (unten) Druckversuch

Druck der maximale Durchmesser zu bestimmen ist (siehe grüne Linie im Auswertebildschirm). Solche Restriktionen lassen sich aber durch vorgefertigte Funktionen innerhalb 
der KEYENCE-Software realisieren. Für die Praxis wäre es jetzt denkbar, Hantelproben aus dem Herstellungsprozess zu entnehmen, diese in die automatische Einspannvorrichtung zu implementieren und Materialeigenschaften mit dem KEYENCE-System zu messen. Dadurch können wichtige Qualitätskontrollen auf Grundlage phänomenologischer Eigenschaften von Elastomeren durchgeführt werden. Als letztes soll überprüft werden, inwieweit sich das Messsystem für dynamische Untersuchungen eignet. In der Abbildung 5.14 ist dazu der Versuchsaufbau einer servo-hydraulischen Prüfmaschine mit dem Probekörper-Setup und dem integrierten KEYENCE-Messsystem dargestellt. Die Untersuchungen haben da-
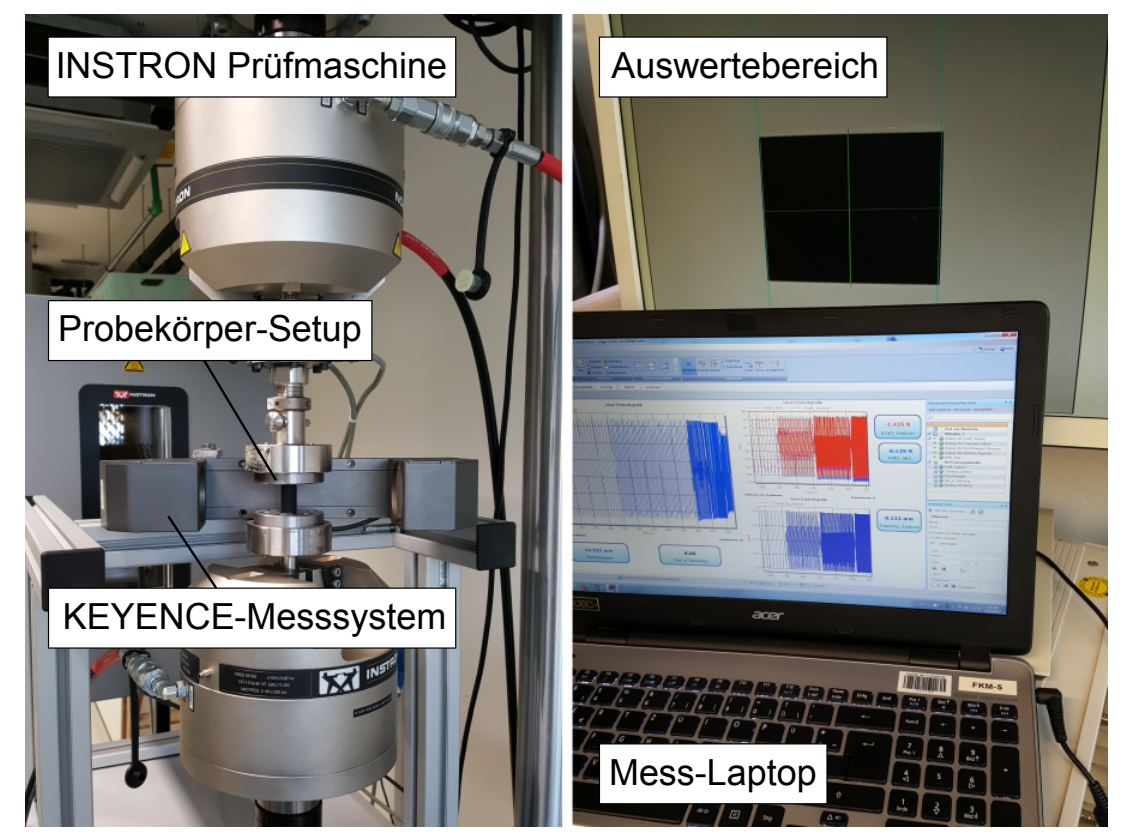

Abb. 5.14 - Versuchsaufbau des Probekörper-Setups an einer servohydraulischen Prüfmaschine mit integriertem KEYENCE-Messsystem

bei ergeben, dass bis zu einer Prüffrequenz von $10 \mathrm{~Hz}$ die Durchmesseränderungen sehr präzise gemessen werden können. Erst bei höheren Prüffrequenzen ergeben sich Probleme mit der Abtastrate. Abhilfe könnte hier ein Mikrometer mit vergrößertem Messbereich schaffen, bei dem die Reduzierung des Auswertebereiches zu einer Erhöhung der Abtastrate führt. Zusammenfassend kann gesagt werden, dass das Probekörper-Setup für die Implementierung in den Industriealltag geeignet ist und gerade im Bereich der Materialcharakterisierung ganz neue Anwendungsmöglichkeiten eröffnet. Beispielsweise wäre gerade für Lebensdaueruntersuchungen das KEYENCE-Messsystem sehr vielversprechend, da hier eine sehr effiziente Messdatenaufzeichnung möglich ist. 



\section{Zusammenfassung und Ausblick}

Elastomerwerkstoffe stellen in vielen technischen Bereichen, beispielsweise in Form von Fahrwerksbuchsen, Dämpfern oder Dichtungen, wichtige Funktionselemente dar. Um grundlegende Eigenschaften technischer Elastomere untersuchen zu können, werden Probekörper benötigt. Die Qualität der Messergebnisse hängt dabei entscheidend von der Homogenität des Messbereiches ab. Für die meisten Standardversuche kommt diesbezüglich eine Zugprobe zum Einsatz (vgl. Brown 2006). Demgegenüber stellt aber gerade die Druckbelastung für viele technische Bauteile eine wichtige Grundbeanspruchungsart dar. Bestehende Probekörperkonzepte zeigen aber schon bei Stauchungen größer 30 \% signifikante Abweichungen gegenüber der homogenen Lösung (vgl. Alshuth u. a. 2007), welche zu erheblichen Fehlern in der Materialcharakterisierung führen. Vor diesem Hintergrund bestand ein Ziel dieser Arbeit in der Entwicklung eines verbesserten Zug-/Druckprobekörpers zur Realisierung homogener Stauchungen. Neben dem einachsigen Zug-/Druckversuch stellt aber auch die einfache Scherung eine weitere wichtige Grundbeanspruchungsart für technische Elastomere dar. Aus diesem Grund erfolgte im Rahmen dieser Arbeit ebenfalls die Entwicklung einer Schervorrichtung für Elastomermatten zur Realisierung präziser Schermessungen.

\section{Ergebnisse dieser Arbeit}

\section{Entwicklung eines Probekörper-Setups für hochpräzise Zug-/Druckmessungen}

Für die Entwicklung eines neuen verbesserten Zug-/Druckprobekörpers findet ein spezielles Design der Halterungskonturen Anwendung. Mit Hilfe eines Berechnungsalgorithmus (vgl. Naumann 2010), bei dem über einen vorgegebenen Eigenfrequenzverlauf in indirekter Weise Einfluss auf die Homogenität und die Sicherheit gegenüber Knicken Bezug genommen werden kann, lassen sich neue Halterungsgeometrien bestimmen. Mittels einer Formoptimierung konnte dabei die ideale Halterungskontur berechnet werden. Da bei Stauchungen größer $50 \%$ der Messbereich sehr limitiert ist, was eine Messung mit Extensometer in axialer Richtung sehr schwierig macht, wurde im Weiteren eine neue Messstrategie für extreme Stauchungen entwickelt. Über die Messung der Umfangsstreckung kann dabei auf eine gemittelte axiale Dehnung geschlossenen werden. Experimentelle Voruntersuchungen und Qualitätsprüfungen haben gezeigt, dass der Probekörper sich nahezu homogen deformieren lässt und hochpräzise Messungen realisiert werden können. Mit dem entwickelten Probekörper-Setup und der neuen Messstrategie lassen sich nun erstmalig Stauchungen 
bis $70 \%$ realisieren und messen. Der Probekörper bietet dabei eine Vielzahl verschiedener Anwendungsmöglichkeiten. Zum einen lassen sich phänomenologische Eigenschaften von Elastomeren vorzugsweise bei großen Stauchungen untersuchen, zum anderen können die nahezu homogenen Messdaten für Stoffgesetzanpassungen verwendet werden. Dadurch können beispielsweise viskoelastoplastische Stoffgesetze wie das MorPH-Modell (vgl. Ihlemann 2003) oder das Rabkin-Modell (vgl. Freund 2013) für einen wesentlich größeren Anwendungsbereich zugänglich gemacht werden. Ein weiterer, ganz praktischer Aspekt besteht darin, dass die Anzahl bestehender Probekörpertypen im Zug-/Druckbereich reduziert werden kann.

\section{Entwicklung einer Schervorrichtung für flächige Elastomermatten zur Realisierung präziser Schermessungen}

Für die Entwicklung eines neuen Scherprobekörpers wurde auf eine stoffschlüssige Verbindung mittels Anvulkanisieren oder Kleben aufgrund von Schrumpf oder Materialirritationen verzichtet (vgl. Ballhorn 2007). Aus diesem Grund lag ein weiterer Schwerpunkt dieser Arbeit in der Entwicklung eines geeigneten Lasteinleitungsdesigns für Elastomermatten zur Realisierung präziser Schermessungen. Als ein sehr vielversprechendes Design stellte sich dabei die Fixierung der Elastomermatte mit dünnen Stahlstiften heraus, die zu einer gleichmäßigen Einleitung der Scherdeformation über der Tiefe beitragen. Anhand von numerischen Untersuchungen konnte gezeigt werden, dass das globale Fehler- und Inhomogenitätsmaß deutlich niedriger ausfällt als bei bisherigen Schervorrichtungen für Elastomermatten. Die experimentelle Versuchsdurchführung mit der neuen Schervorrichtung bestätigt dieses Ergebnis und ermöglicht nun ganz neue Anwendungsmöglichkeiten im Bereich der Materialcharakterisierung. Außer der phänomenologischen Untersuchung grundlegender Eigenschaften können nun auch Versuche mit gealterten oder faserverstärkten Elastomermatten durchgeführt werden. Weiterhin wurde das Beulverhalten bei großen Scherungen für verschiedene Mattendicken charakterisiert. Dabei zeigte sich eine gute Übereinstimmung zwischen Simulation und Experiment. Mit Hilfe dieser Erkenntnisse kann der Scherprobekörper in weiterführenden Arbeiten hinsichtlich Homogenität und Sicherheit gegenüber Beulen optimiert werden.

\section{Phänomenologische Charakterisierung technischer Elastomere und Stoffgesetzanpassungen}

Mit den entwickelten homogenen Probekörpern für Zug-/Druck und Scherung lassen sich viele wichtige Eigenschaften untersuchen, wie zum Beispiel: Payne-Effekt, Mullins-Effekt, anisotrope Entfestigung, bleibende Verzerrungen, Relaxations- und Kriechverhalten für technische Elastomere. In dieser Arbeit wurde weiterhin eine externe Sollwertsteuerung für eine Zwick/Roell Zug-/Druckprüfmaschine entwickelt, mit der sämtliche nur denkbare Funktionsverläufe im Rahmen der physikalischen Möglichkeiten der Maschine verfahren 
werden können. Damit wurde ein Werkzeug zur Verfügung gestellt, mit dem das ratenabhängige Materialverhalten von technischen Elastomeren in sehr präziser Weise untersucht werden konnte. Dabei zeigten sich sehr interessante geschwindigkeitsabhängige Effekte, die für die Validierung ratenabhängiger Stoffgesetzformulierungen verwendet werden können (vgl. Donner u. a. 2017). Im Weiteren wurde ein viskoelastoplastisches Stoffgesetz mit Zug/Druckdaten angepasst und eine Probekörpersimulation zur Abschätzung des Messfehlers durchgeführt. Dabei stellte sich heraus, dass der Fehler zwischen Anpassung und Probekörpersimulation mit der neuen Messstrategie sehr gering ausfällt, was die hohe Güte des Zug-/Druckprobekörpers bestätigt. Mit der Implementierung des Probekörper-Setups in den Industriealltag findet die Entwicklung einen erfolgreichen Abschluss.

\section{Ausblick}

\section{Lebensdaueruntersuchungen}

Für den neu entwickelten Zug-/Druckprobekörper kann gezeigt werden, dass im Druckversuch die maximalen Spannungen im Messbereich auftreten. Eine Erklärung hierfür ist das spezielle Design der Halterungskonturen, welches zu einer nahezu homogenen Bauteildeformation beiträgt. Diese Maximalbeanspruchung im Messbereich prädestiniert den Zug-/Druckprobekörper in idealer Weise für Lebensdaueruntersuchungen. In einer ersten Versuchsserie im Zugschwellbereich konnte mit dem neuen Probekörper-Setup bereits bestätigt werden, dass die maximale Schädigung (Rissentstehung und Wachstum) im Messbereich auftritt. In weiterführenden Lebensdaueruntersuchungen soll der Probekörper für den Wechselbereich und den Druckschwellbereich eingesetzt werden. Dieser erweiterte Messbereich bietet insbesondere für die Entwicklung neuer Lebensdauerhypothesen vielfältige Anwendungsmöglichkeiten. Beispielsweise wäre es denkbar, den Probekörper auf unterschiedliche Druckstufen anzufahren und dann mit einer kraftgesteuerten Belastung fortzufahren. Darauf aufbauend ergeben sich eine Reihe interessanter Fragestellungen, wie die der Rissentstehung, der Rissausbreitung und der Versagensmechanismen im Druckbereich. In der Veröffentlichung von Abraham u. a. (2001) finden sich Lebensdaueruntersuchungen mit der bisherigen Standard-Hantel und der vorliegenden Halterung, allerdings nur für den Zugbereich.

\section{Alterungsuntersuchungen}

Ein weiterer Anknüpfungspunkt für spätere Arbeiten könnte die Miniaturisierung des Probekörper-Setups für homogene Alterungsuntersuchungen sein. Beispielsweise hätte ein skalierter Zug-/Druckprobekörper den großen Vorteil, dass der Sauerstoff gleichmäßig in das Innere des Probekörpers diffundieren kann und sich dadurch eine weniger inhomogen gealterte Grenzschicht ausbildet. Im Anhang A.6 ist zur besseren Veranschaulichung das Probekörper-Setup und ein weiteres, um den Faktor 5 skaliertes, dargestellt. Nach verschiedenen Alterungsphasen könnten dann zyklische Druckversuche gefahren werden, um 
die Phänomenologie gealterter Elastomerproben zu charakterisieren. Diese Erkenntnisse stellen eine wichtige Grundlage für die Weiterentwicklung bestehender Alterungsmodelle von Elastomeren dar (vgl. Naumnann 2016). Neben den fertigungsbedingten Herausforderungen ist der Einfluss von Imperfektionen vermutlich die größte Schwierigkeit. Hierbei ist zu beachten, dass Störungen in Form von Materialinhomogenitäten oder Geometrieimperfektionen sich bei einer Miniaturisierung nicht mit skalieren. Demgegenüber wären Lebensdaueruntersuchungen mit unterschiedlich skalierten Hantelproben sehr reizvoll, da hier der Einfluss von Fehlstellen auf das Versagen untersucht werden könnte.

\section{Untersuchung faserverstärkter Materialien}

Die experimentelle Charakterisierung faserverstärkter Materialien anhand der entwickelten Probekörper ist gleichfalls ein interessanter Aspekt. Beispielsweise wäre es denkbar in der Vulkanisationsform des Hantelprobekörpers einzelne Garne bzw. Hybridcorde einzubetten (Corde müssten zwischen den Hantelköpfen verspannt werden), bevor das Elastomer eingespritzt wird. Damit könnten wichtige Untersuchungen im Zug-/Druckbereich durchgeführt werden, um Materialkennwerte für Reifen und Luftfedern zu bestimmen (vgl. Donner 2017). Eines der Hauptprobleme in der Modellierung faserverstärkter Strukturen ist der große Steifigkeitsunterschied zwischen weichem Elastomer und steifem Hybridcord. Das neu entwickelte Probekörper-Setup könnte hier signifikante Vorteile für experimentelle Untersuchungen aufweisen. Mit der Schervorrichtung ließen sich dann weiterhin faserverstärkte Elastomermatten testen. Diese Ergebnisse könnten dann den Zug-/Druckversuchen gegenübergestellt werden. Ein 3D DIC-Messsystem hätte hier den ganz praktischen Vorteil, dass der inhomogene Messbereich (Übergang zwischen Cord und Elastomer) sehr präzise ausgewertet werden könnte. 


\section{A Anhang}

\section{Anhang zur Optimierung der Probekörperlänge}

In Abbildung A.1 und A.2 ist der Zielfunktionsverlauf für identische asymptotische Eigenfrequenzvorgaben der unterschiedlichen Parameterkonstellationen dargestellt. Die Abbildungen zeigen, dass eine Variation dieser Parameter zu keiner signifikanten Änderung des Zielfunktionsverlaufes führt.

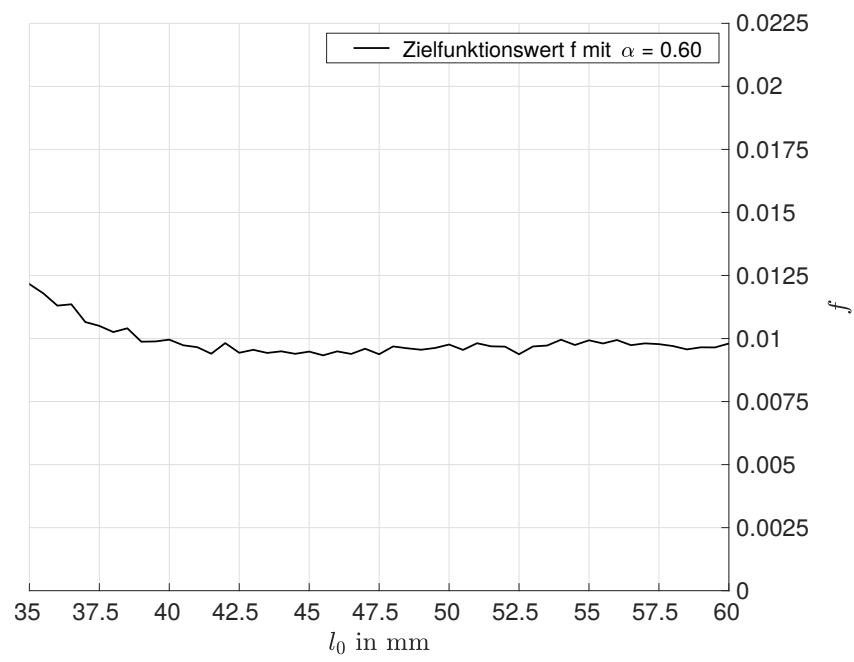

Abb. A.1 - Darstellung der Zielfunktion $f$ für identische asymptotische Eigenfrequenzvorgaben $l_{0}=50 \mathrm{~mm}$, Schnittpunkt $\varepsilon=-0.4$ (Simulationsergebnis)

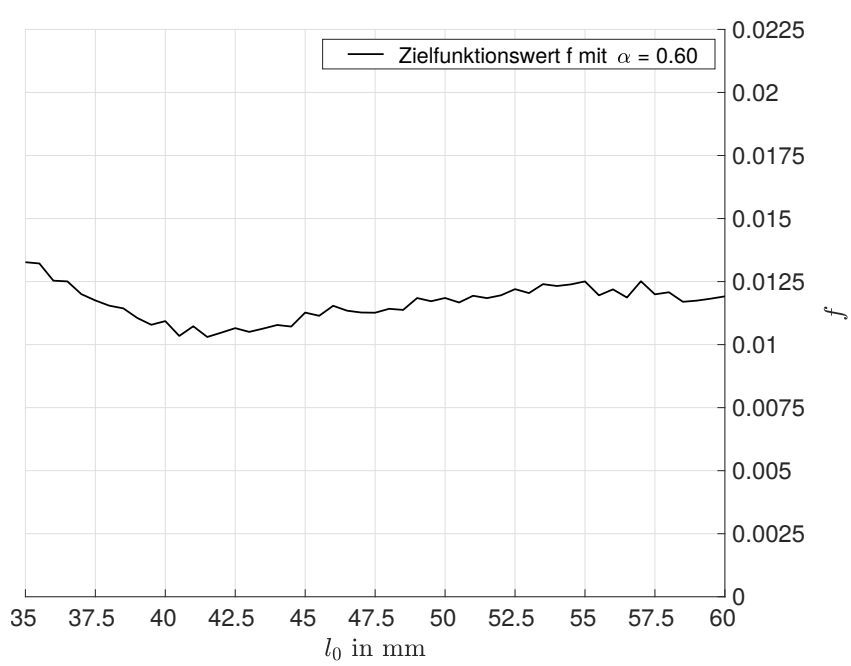

Abb. A.2 - Darstellung der Zielfunktion $f$ für identische asymptotische Eigenfrequenzvorgaben $l_{0}=55 \mathrm{~mm}$, Schnittpunkt $\varepsilon=-0.3$ (Simulationsergebnis) 


\section{Anhang zur Beuluntersuchung für den idealisierten Scherprobekörper}

In Abbildung A.3 ist das Beulverhalten für den idealisierten Scherprobekörper bei einer Winkelimperfektion von $\varphi=2.5^{\circ}$ dargestellt.

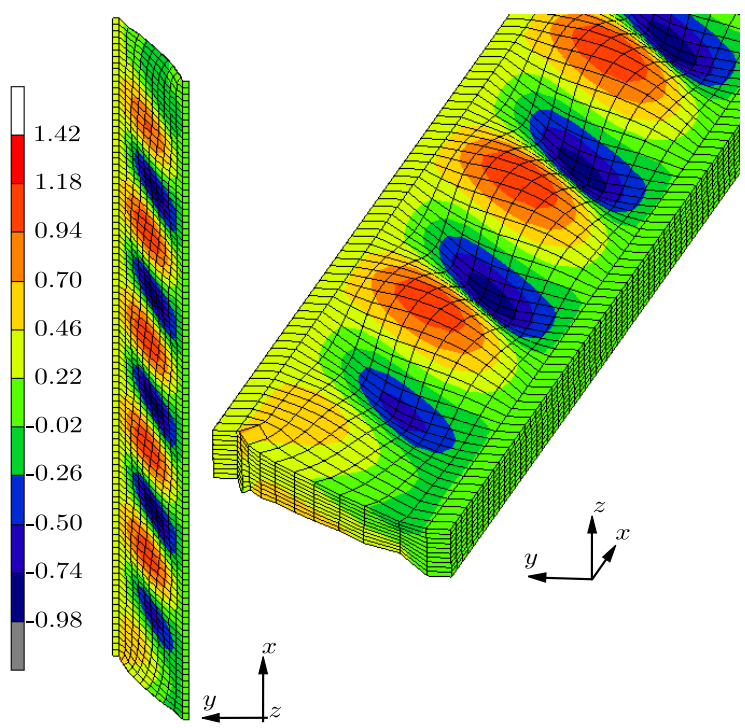

Abb. A.3 - Darstellung der $z$-Verschiebung für den idealisierten Scherprobekörper mit den Abmaßen: $10 \times 100 \times 2$ bei einer Winkelimperfektion von $\varphi=2.5^{\circ}$

\section{Anhang zur numerischen Entwicklung eines Scherprobekörpers mit formschlüssiger Lasteinleitung}

In Abbildung A.4 ist der neu entwickelte Scherprobekörper mit formschlüssiger Lasteinleitung dargestellt.

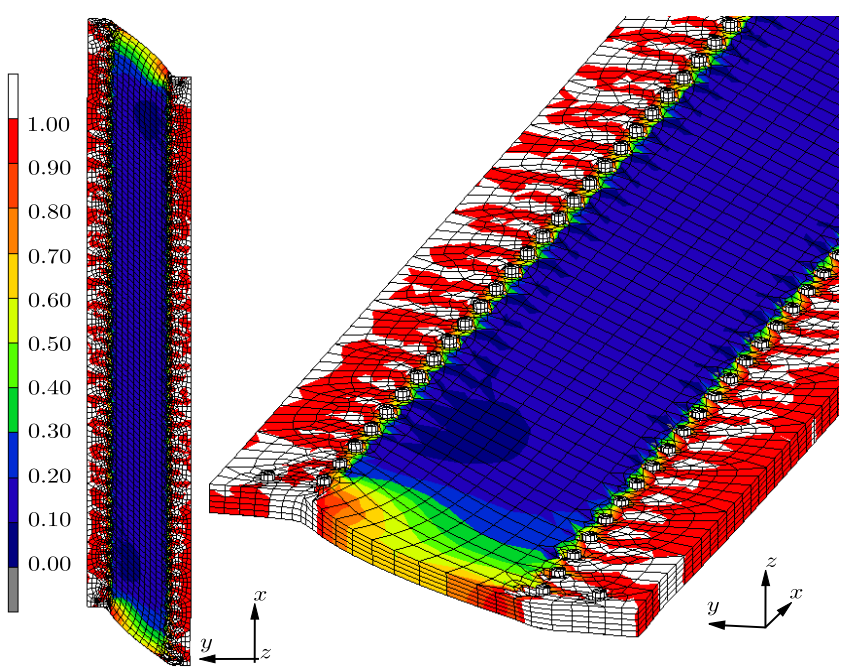

Abb. A.4 - Darstellung des lokalen Fehlermaßes $\eta_{\mathrm{s}}$ für den neuen Scherprobekörper mit den Abmaßen: $10 \times 100 \times 2$ mit Pin-Durchmesser $d=0.5 \mathrm{~mm}$ bei einer Scherbelastung von $u_{x}=10 \mathrm{~mm}$ (Symmetrie in negativer $z$-Richtung) 


\section{Anhang zur Untersuchung des ratenabhängigen Materialverhaltens von Elastomeren}

In Abbildung A.5 ist das Spannungs-Schermaßdiagramm für einen verzerrten Sinusverlauf (Sägezahnverlauf) zur Untersuchung des ratenabhängigen Materialverhaltens für den einfachen Scherversuch dargestellt.

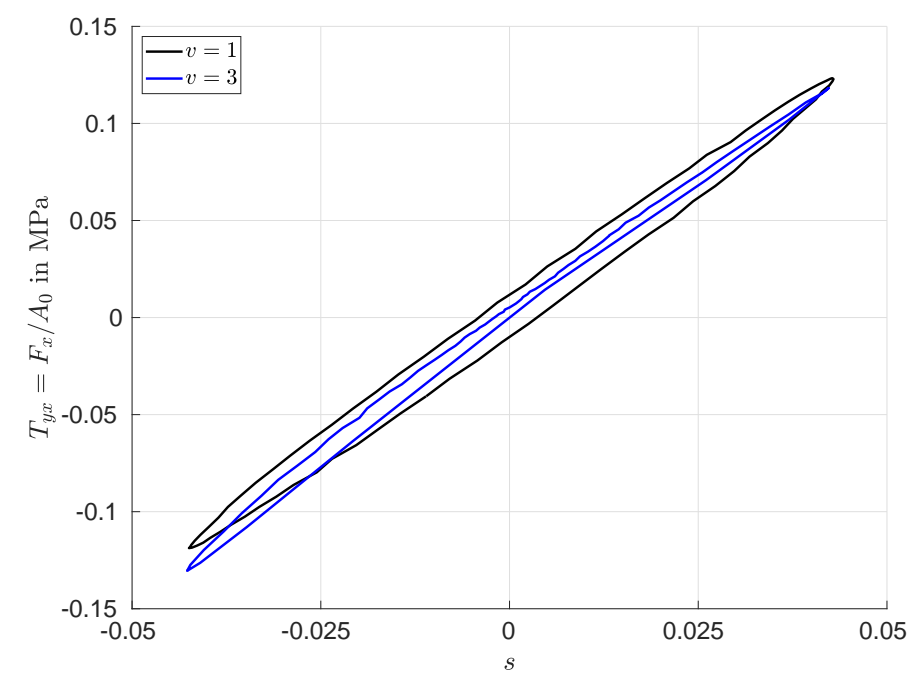

Abb. A.5 - Spannungs-Schermaßantwort des verzerrten Sinusverlaufes (Sägezahnverlauf) für den einfachen Scherversuch (Messungen für gefüllten EPDM)

\section{Anhang zur Miniaturisierung des Probekörper-Setups}

In Abbildung A.6 ist die Miniaturisierung des Probekörper-Setups um den Faktor 5 für homogene Alterungsuntersuchungen dargestellt.

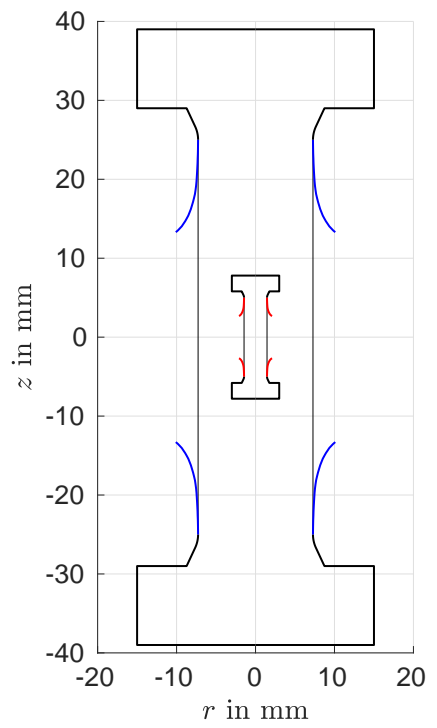

Abb. A.6 - Miniaturisierung des Probekörper-Setups für homogene Alterungsuntersuchungen (Simulationsergebnisse) 



\section{Literatur}

Abraham, F., T. Alshuth \& S. Jerrams (2001). Ermüdungsbeständigkeit von Elastomeren Einfluss der Spannungsamplitude und der Unterspannung. KGK. Kautschuk, Gummi, Kunststoffe 54 (12), S. 643-647.

Ahmadi, H.R., D. Besdo, J. Ihlemann, J.G.R. Kingston \& A.H. Muhr (2005). Transient response of inelastic materials to changes of amplitude. Constitutive Models for Rubber IV. Hrsg. von P.-E. Austrell \& L. Kari. Leiden: Balkema, S. 305-312.

Ahmadi, H.R., J. Ihlemann \& A.H. Muhr (2007). Time-dependent effects in dynamic tests of filled rubber. Constitutive Models for Rubber V. Hrsg. von A. Boukamel, L. Laiarinandrasana, S. Méo \& E. Veron. London: Taylor \& Francis Group, S. 305-310.

Alshuth, T., C. Hohl \& J. Ihlemann (2007). Vergleichende Messungen und Simulationen annähernd homogener Belastungsverteilungen. PAMM 7 (1), S. 53-54.

Ballhorn, M. (2007). Schadensanalyse an Gummi/Metall-Bauteilen. KGK. Kautschuk, Gummi, Kunststoffe 60 (5), S. 249-256.

Bergström, J. S. (1999). Large strain time-dependent behavior of elastomeric materials. Massachusetts Institute of Technology.

Besdo, D., J. Ihlemann, J.G.R. Kingston \& A.H. Muhr (2003). Modelling inelastic stressstrain phenomena and a scheme for efficient experimental characterization. Constitutive Models for Rubber III. Hrsg. von J.J.C. Busfield \& A.H. Muhr. Lisse: Balkema, S. 309318.

Bronstein, I. N., K. A. Semendjajew, G. Musiol \& H. Mühlig (2012). Taschenbuch der Mathematik. Verlag Harri Deutsch.

Brown, R. (2006). Physical Testing of Rubber. Springer US.

Donner, H. (2017). FEM-basierte Modellierung stark anisotroper Hybridcord-ElastomerVerbunde. Dissertation. Chemnitz: TU Chemnitz.

Donner, H., L. Kanzenbach, J. Ihlemann \& C. Naumann (2017). Efficiency of rubber material modelling and characterisation. Constitutive Models for Rubber X. Hrsg. von A. Lion \& M. Johlitz. London: Taylor \& Francis Group, S. 19-29.

Dresig, H., F. Holzweißig \& L. Rockhausen (2007). Maschinendynamik. Berlin Heidelberg: Springer-Verlag.

Freund, M. (2007). FE-gestütze Entwicklung einer für Lebensdauerversuche unter Scherbelastung geeigneten Geometrie für Elastomerprüfkörper. Masterarbeit. Hannover: Leibniz Universität Hannover. 
Freund, M. (2013). Verallgemeinerung eindimensionaler Materialmodelle für die FiniteElemente-Methode. Düsseldorf: VDI-Verlag.

Gelke, S. \& J. Ihlemann (2016). Simulation of a chassis bushing with regard to strain induced softening of filled rubber. PAMM 16 (1), S. 339-340.

Gross, D., W. Hauger \& P. Wriggers (2007). Technische Mechanik - Band 4: Hydromechanik, Elemente der Höheren Mechanik, Numerische Methoden. Berlin Heidelberg: Springer-Verlag.

Hagedorn, P. \& A. DasGupta (2007). Vibrations and Waves in Continuous Mechanical Systems. Chichester: John Wiley \& Sons Verlag.

Hartmann, S. (2003). Finite-Elemente Berechnung inelastischer Kontinua. Interpretation als Algebro- Differentialgleichungssysteme. Habilitationsschrift, Universität Kassel.

Haupt, P. (2002). Continuum Mechanics and Theory of Materials. Berlin Heidelberg: Springer-Verlag.

Heinrich, G., E. Straube \& G. Helmis (1988). Rubber elasticity of polymer networks: Theories. Berlin Heidelberg: Springer-Verlag.

Holzapfel, G. A. (2000). Nonlinear Solid Mechanics. Chichester: John Wiley \& Sons Verlag.

Ihlemann, J. (2003). Kontinuumsmechanische Nachbildung hochbelasteter technischer Gummiwerkstoffe. Düsseldorf: VDI-Verlag.

Ihlemann, J. (2014). Beobachterkonzepte und Darstellungsformen der nichtlinearen Kontinuumsmechanik. Düsseldorf: VDI-Verlag.

Johlitz, M. \& S. Diebels (2011). Characterisation of a polymer using biaxial tension tests. Part I: Hyperelasticity. Archive of Applied Mechanics 81 (10), S. 1333-1349.

Kanzenbach, L., M. Stockmann \& J. Ihlemann (2016a). Extension of the Testing Machine Control for High-precision Uniaxial Tension Measurements. Materials Today: Proceedings 3 (4), S. 993-996.

Kanzenbach, L., C. Naumann \& J. Ihlemann (2016b). Specimen design for high precision tension-compression tests. PAMM 16 (1), S. 207-208.

Kanzenbach, L., R. Kießling, M. Stockmann \& J. Ihlemann (2017). Development of a compression chamber for the determination of the bulk modulus. Materials Today: Proceedings 4 (5), S. 5839-5842.

Kanzenbach, L., E. Oelsch, M. Stockmann \& J. Ihlemann (2018). A new specimen setup for high precision uniaxial tension-compression tests of rubber. Materials Today: Proceedings 5 (6), S. 26747-26752.

Kanzenbach, L., E. Oelsch, T. Lehmann \& J. Ihlemann (2019a). Dynamic testing of a specimen setup for combined high precision uniaxial tension-compression tests of rubber. Materials Today: Proceedings 12, S. 383-387.

Kanzenbach, L., C. Schlomka, S. Gelke \& J. Ihlemann (2019b). Specimen design for extreme uniaxial tension-compression tests of rubber materials. PAMM 19 (1), e201900371. 
Klauke, R. (2015). Lebensdauervorhersage mehrachsig belasteter Elastomerbauteile unter besonderer Berücksichtigung rotierender Beanspruchungsrichtungen. Dissertation. Chemnitz: TU Chemnitz.

Kottner, R., J. Heczko \& J. Krystek (2018). Validation of identified material parameters of rubber using an arcan shear test. Editura Printech, S. 79-80.

Krawietz, A. (1986). Materialtheorie: Mathematische Beschreibung des phänomenologischen thermomechanischen Verhaltens. Berlin Heidelberg: Springer-Verlag.

Kröner, E. (1958). Allgemeine Kontinuumstheorie der Versetzungen und Eigenspannungen. Berlin: Springer.

Lampe, A., L. Kanzenbach, J. Ihlemann \& A.H. Muhr (2018). Characteristics of relaxation and recovery behavior of filled rubber. KGK. Kautschuk, Gummi, Kunststoffe 71 (10), S. $47-53$.

Lei, Y., T. Murmu, S. Adhikari \& M. Friswell (2013). Dynamic characteristics of damped viscoelastic nonlocal Euler-Bernoulli beams. European Journal of Mechanics - A/Solids 42, S. $125-136$.

Lion, A. (2000). Thermomechanik von Elastomeren: Experimente und Materialtheorie. Kassel: Gesamthochschul-Bibliothek.

Msc. Software (2017). Volume A: Theory and User Information. Msc. Software Corporation.

Mullins, L. (1948). Effect of stretching on the properties of rubber. Rubber Chemistry und Technology 21 (2), S. 281-300.

Naumann, C. (2010). Virtuelle Geometrieoptimierung eines Laborprüfkörpers für kombinierte Zug-/Druckbelastungen. Studienarbeit. Chemnitz: TU Chemnitz, unveröffentlicht.

Naumnann, C. (2016). Chemisch-mechanisch gekoppelte Modellierung und Simulation oxidativer Alterungsvorgänge in Gummibauteilen. Dissertation. Chemnitz: TU Chemnitz.

Oelsch, E. (2017). Experimentelle Untersuchungen zur phänomenologischen Charakterisierung technischer Elastomere. Masterarbeit. Chemnitz: TU Chemnitz, unveröffentlicht.

Pahr, D. H. \& P. K. Zysset (2008). Influence of boundary conditions on computed apparent elastic properties of cancellous bone. Biomechanics und modeling in mechanobiology 7 (6), S. 463-476.

Palmov, V. (1998). Vibrations of Elasto-Plastic Bodies. Berlin Heidelberg: Springer-Verlag.

Rivlin, R. S. \& D. W. Saunders (1951). Large elastic deformations of isotropic materials. VII. Experiments on the deformation of rubber. Philosophical Transactions of the Royal Society of London. Series A, Mathematical und Physical Sciences 243 (865), S. 251-288.

Schellenberg, D. (2017). Identifikation und Optimierung im Kontext technischer Anwendungen. Dissertation. Chemnitz: TU Chemnitz.

Schellenberg, D., D. Juhre \& J. Ihlemann (2012). Parameter identification based on multiple inhomogeneous experiments of practical relevance. PAMM 12 (1), S. 629-630. 
Seibert, H., T. Scheffer \& S. Diebels (2014). Biaxial testing of elastomers-Experimental setup, measurement and experimental optimisation of specimen's shape. Technische Mechanik 34 (2), S. 72-89.

Stańco, M. \& P. Działak (2018). Numerical and experimental analysis of the rubber bumper stiffness. Editura Printech, S. 37-38.

Staudinger, U. (2007). Morphologie und Bruchverhalten von Block- und Multipfropfcopolymeren. Dissertation. Dresden: TU Dresden.

Stelzmann, U., G. Müller \& C. Groth (2002). FEM für Praktiker - Band 2: Strukturdynamik. Expert-Verlag 44.

Stommel, M. \& J. Zimmermann (2011). Determination of the behaviour of rubber components under hydrostatic pressure. Constitutive Models for Rubber VII, S. 397.

Sussman, T. \& K.-J. Bathe (1987). A Finite Element Formulation for Nonlinear Incompressible Elastic and Inelastic Analysis. Computers and Structures 26, S. 357-409.

Sutton, M. A., J. J. Orteu \& H. Schreier (2009). Image Correlation for Shape, Motion and Deformation Measurements. Springer US.

Tölle, F. J., M. Gräfen, L. Kanzenbach \& J. Ihlemann (2018). Static and Dynamic Characterization of Rubber without Metal Bonding. Deutsche Kautschuk-Tagung, Konferenzbeitrag unveröffentlicht.

Virgin, L. N. (2007). Vibration of Axially-Loaded Structures. Cambridge University Press.

Wriggers, P. (2001). Nichtlineare Finite-Element-Methoden. Berlin Heidelberg: SpringerVerlag.

Wulf, H. (2016). Modellierung und Simulation von Selbstorganisationsprozessen in belasteten technischen Gummiwerkstoffen. Dissertation. Chemnitz: TU Chemnitz.

Zimmermann, J. \& M. Stommel (2013). The mechanical behaviour of rubber under hydrostatic compression and the effect on the results of finite element analyses. Archive of Applied Mechanics 83 (2), S. 293-302. 


\section{Lebenslauf}

Lars Kanzenbach

$\begin{array}{ll}\text { Geburtsdatum } & \text { 30. Januar } 1989 \\ \text { Geburtsort } & \text { Prenzlau }\end{array}$

Schulausbildung

1995-1999

Grundschule Penkun

1999-2000

Realschule Penkun

2000-2008

Europaschule Deutsch-Polnisches Gymnasium Löcknitz,

Abschluß Abitur

\section{$\underline{\text { Studium }}$}

2008-2011

Maschinenbaustudium an der TU Chemnitz

Abschluß: Bachelor im September 2011

2011-2013

Maschinenbaustudium an der TU Chemnitz

Abschluß: Master im Oktober 2013

Berufstätigkeit

seit 2013

Wissenschaftlicher Mitarbeiter an der Professur Festkörpermechanik 